\title{
Building Resilience in At-Risk Adolescents: Comparing the mechanisms and outcomes of two school-based prevention programmes.
}

\author{
By Olivia Susanne Notter
}

\author{
A thesis \\ submitted to the Victoria University of Wellington \\ in fulfilment of the requirements for the degree of \\ Doctor of Philosophy
}

Victoria University of Wellington

2013 


\begin{abstract}
This study sought to confirm and expand literature on psychological health by comparing and contrasting the effects of two prevention programmes, one focused on reducing negative affect and the other focused on enhancing positive affect, and by revealing possible pathways that might lead to increased wellbeing and resilience and reduced negative affect and depressive symptoms.

Two school-based intervention approaches were examined: Kiwi ACE (Adolescents Coping with Emotions) and PAL (Positive Approaches to Life), to investigate which techniques would prevent the occurrence of depression, increase wellbeing, and help build resilience in Year 9 students (13-yr-olds). Kiwi ACE is a programme based on a CBT (Cognitive-Behavioural Therapy) model and was trialled previously with success. PAL kept within the same structure as Kiwi ACE but used strategies aimed at building a capacity within students to develop positive emotions in order to build resources for present and future challenges. Both programmes aimed to build resilience and prevent the development of depression in at-risk adolescents.

Nine schools from the wider Wellington region in New Zealand participated in the current study. Sixty-five students identified as at-risk, participated in one of the two programmes, and 69 students constituted the control group. All students in the current study were at risk of developing depression, pre-intervention, as suggested by a mild-moderate score on the CDI (Children's Depression Inventory). Both programmes consisted of weekly one hour sessions enacted over 12 weeks during which a group of approximately 10 students met with a clinical psychologist and school counsellor. A survey consisting of a range of scales, measured students' scores pre- and post- programme, at six months and one year after the programme was completed.

Kiwi ACE and PAL both decreased depressive symptoms and increased well-being for up to one year after the programme. However, PAL had stronger effects in promoting gratitude, satisfaction with life, happiness, and resilience. Mediation analysis revealed that Kiwi ACE helped to decrease depressive symptoms by increasing students' sense of environmental mastery and increased students' well-being scores by decreasing the intensity and frequency of participants' negative emotions. In contrast, PAL helped to decrease depressive symptoms, and increase well-being and resilience through many routes, namely through increasing gratitude, meaning, happiness and satisfaction with life.
\end{abstract}


The findings of this study reveal that building a capacity for positive emotions can help develop many resources that protect students from depressive symptoms and increase their psychological well-being and personal resilience. In addition, using positive emotions as a resource is equally effective as using CBT strategies in preventing depression and is more effective in increasing positive outcomes, including personal resilience. Finally the results from PAL indicate that cultivating positive emotions such as gratitude and happiness is an effective way to build personal resilience in adolescence. 


\section{Acknowledgements}

Undertaking this project has been a valuable journey of learning, passion and perseverance. The whole process, from the development of the programmes to writing the last conclusion, was made possible by a wide range of people and organisations offering their services, support, advice and resources. Firstly, I acknowledge and thank the schools, their principals and deans for allowing these programmes to be implemented, and helping to organise the repetitive surveying that took place throughout the year. I am extremely grateful to all the school counsellors, as without them the whole project would not have been possible. I especially appreciate the obvious concern they have for the welfare of their students and the zeal they displayed in implementing both programmes.

I owe thanks to Compass Health for providing two psychologists from Gains Psychology to help run the programmes. I am very appreciative of the teaching, care and precision provided by the psychologists and enjoyed working alongside them. Dr Barabara Woods was a key player in making schools aware of Kiwi ACE and facilitating their participation. I have Barbara to thank for kick-starting the whole project and connecting me with the schools. Additionally Barbara played a supportive role in the practicalities of running the programme and providing a class to trial PAL. I cannot continue without thanking the New Zealand Lottery Grants Board and Lottery Health Research Committee to acknowledge the Scholarship I received from them in the second year of my thesis. This scholarship made it possible for me to wholly focus on my research and complete it efficiently and with quality and to contribute a valuable resource for the benefit of the community.

I feel very fortunate to have had Associate Professor Paul Jose as my supervisor. His teaching, support and encouragement has been invaluable. I am extremely appreciative of all the quality time and energy Paul put into this project and the many valuable tools and skills he taught me along the way. I have appreciated all the advice offered to me and discussions we have had regarding statistics, methodology and writing. I value Paul's patience, teaching and acceptance and hope some of his talent has rubbed off on me.

I also thank my friends Melissa Grouden, Denise Kirby and Bee Lim for their support and friendship and those many discussions related to, or not related to, my thesis, but helped all the same to keep my wellbeing in check and focus on what was important. I owe thanks to John Bell, for all the hours that he put into editing the programme manuals and group books, and the advice and support he gave through PAL's creation. I am honoured to be friends with 
Sheryl Boxall, who has continuously given me valuable advice, support and edited my work throughout my academic life. Sheryl has shaped how I see the world and has helped me accomplish more than I ever set out to do simply by believing in me. Also, thanks to my family for their love and financial support. Finally, the love of my life, Jarod Campbell, he has made me feel like a champion when I wanted to give up. I am grateful for the editing, and the continued understanding and support I received from him, especially in the last few months of finishing my thesis when it completely consumed my life. Without all these people I would have not been able to create this project or complete it. I am grateful and honoured to have been a part of something that will hopefully continue to make a positive difference in young peoples' lives. 


\section{Table of Contents}

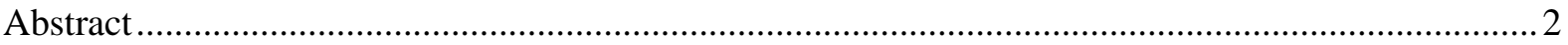

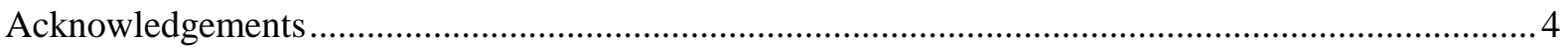

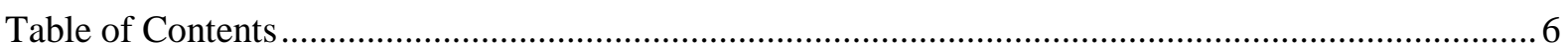

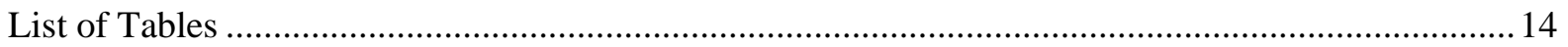

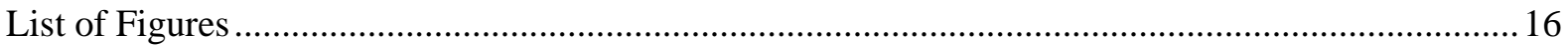

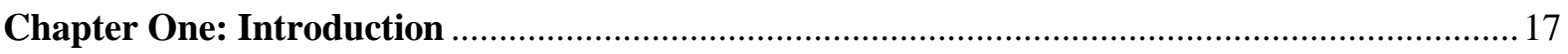

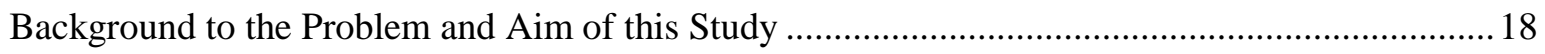

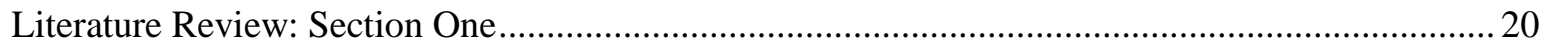

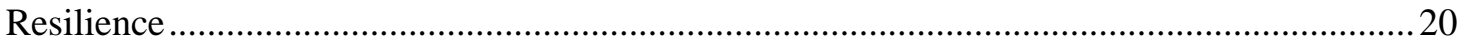

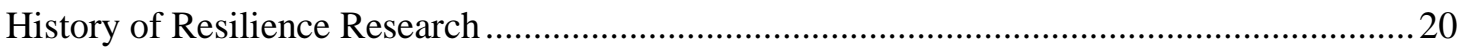

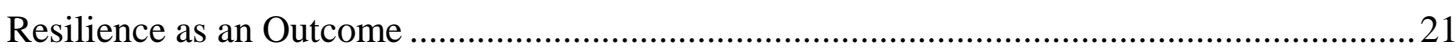

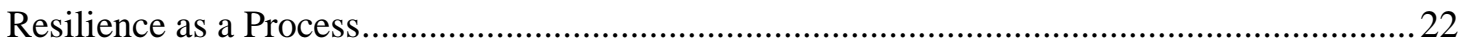

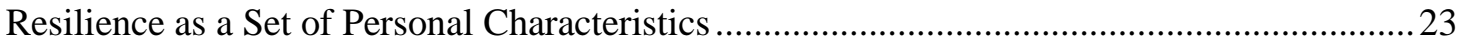

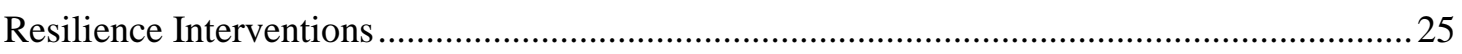

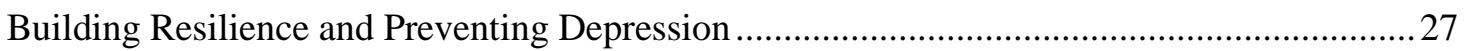

School-based Preventative Programmes: Universal, Selective and Indicated ............................28

Cognitive-Behaviour Models of Prevention Programmes .............................................................. 30

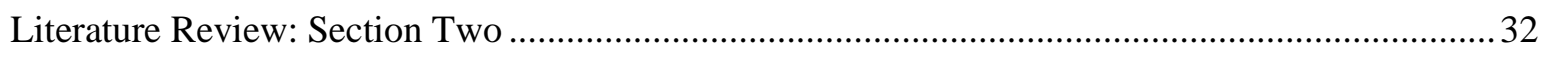

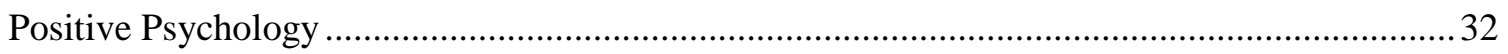

Positive Psychology's Contribution to Resilience ....................................................................... 34

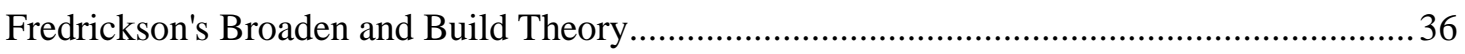

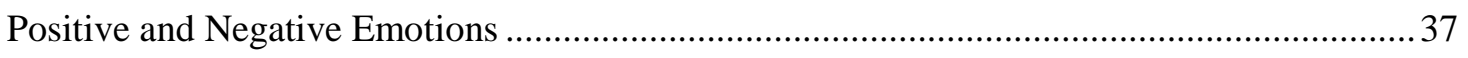

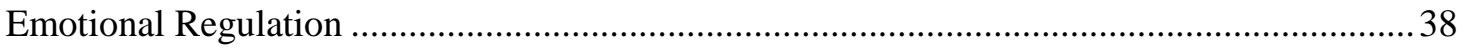

Happiness, Satisfaction with Life and Psychological Well-being ............................................ 39

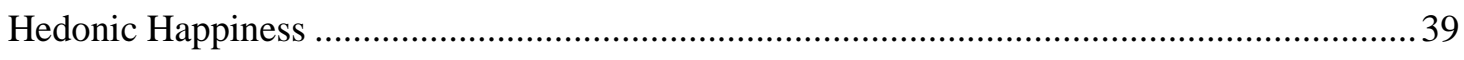

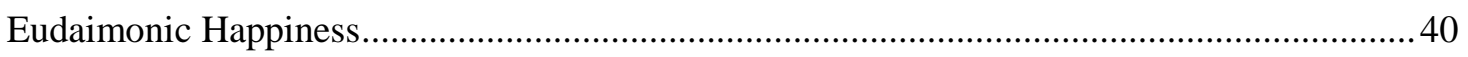

It is Possible to Increase Sustainable Happiness and Wellbeing?............................................. 41 


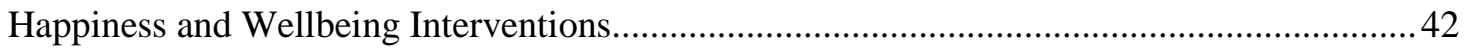

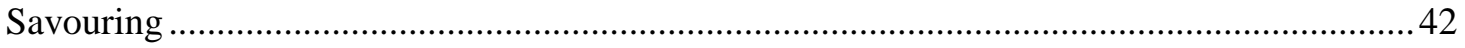

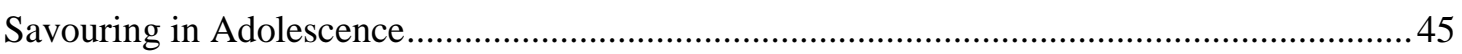

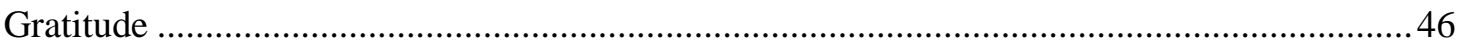

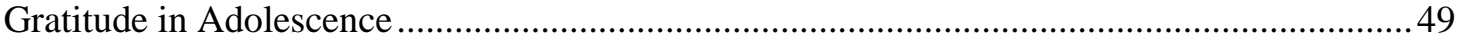

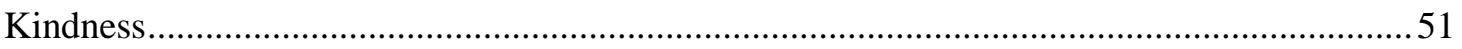

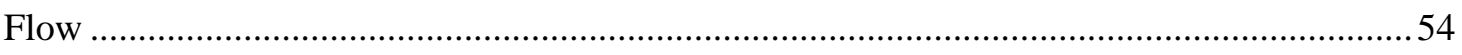

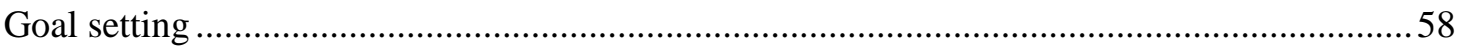

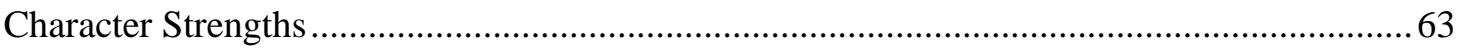

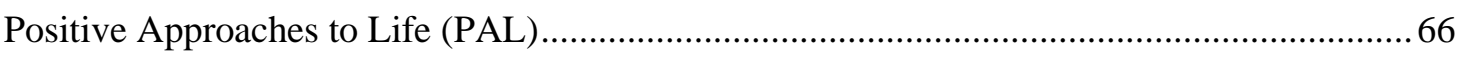

Gaps in Current Positive Psychology and Prevention Programmes ............................................. 66

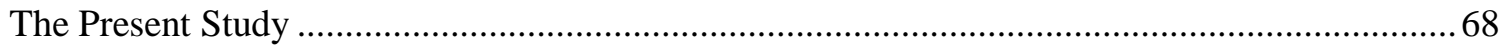

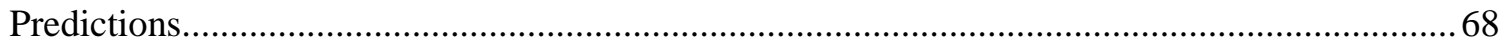

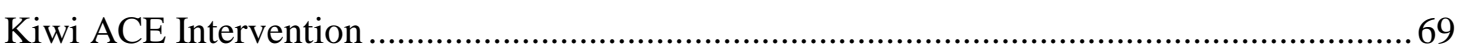

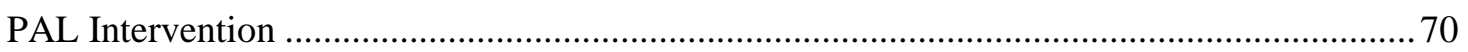

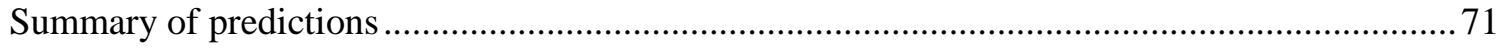

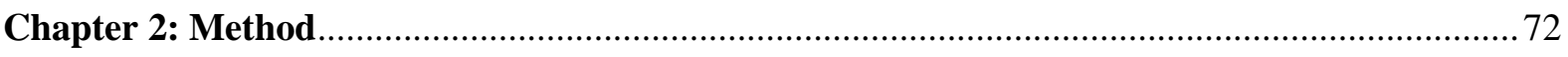

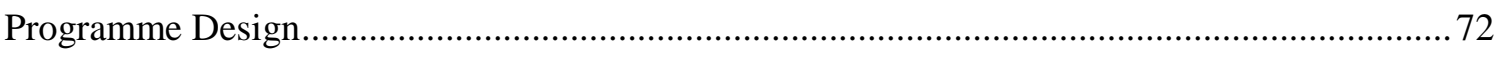

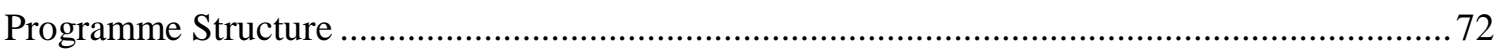

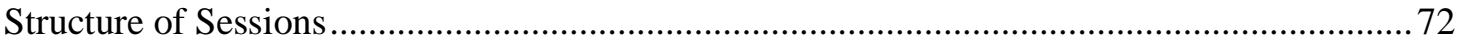

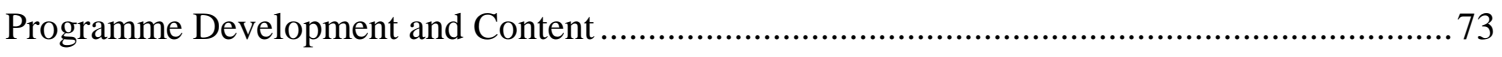

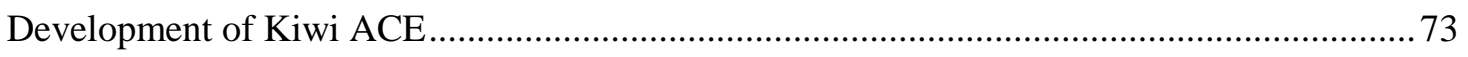

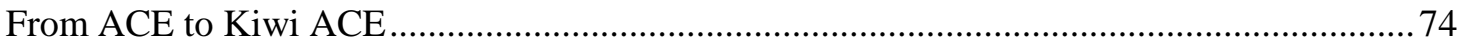

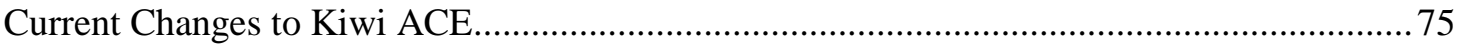

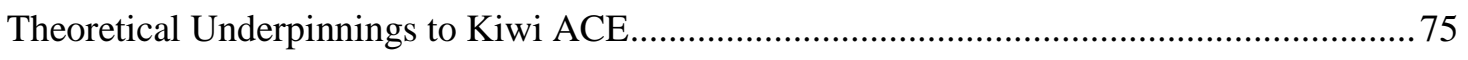

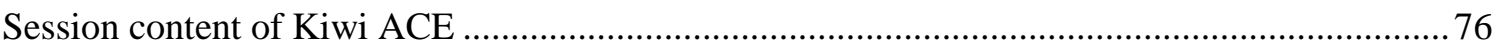

Session One: Introduction - Stress and My Body .............................................................. 76

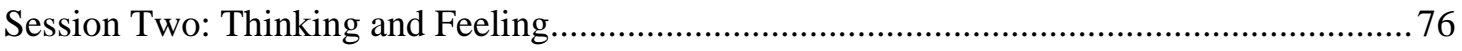


Session Three: Thoughts aren't Facts

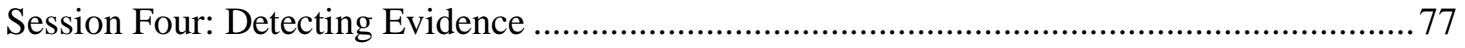

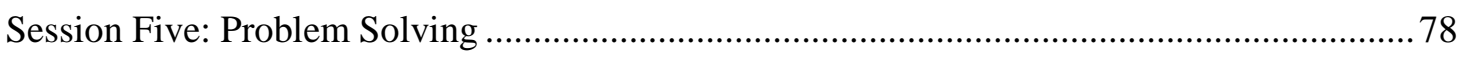

Session Six: Thinking Errors and Changing Unhelpful Habits................................................ 79

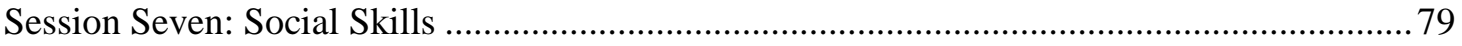

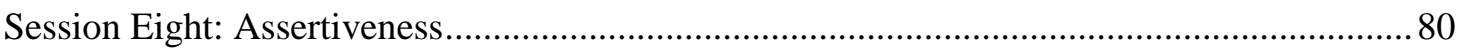

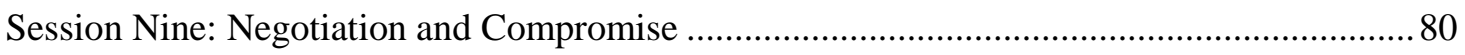

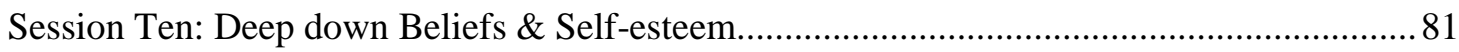

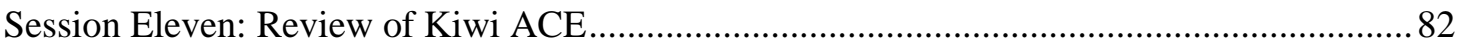

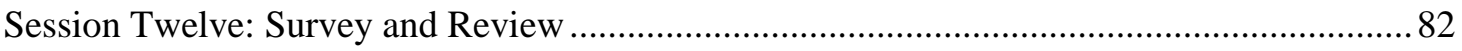

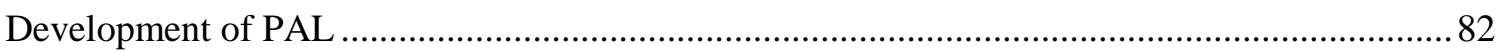

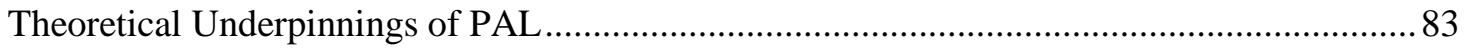

Content of PAL Sessions - Differences from Kiwi ACE ............................................................. 83

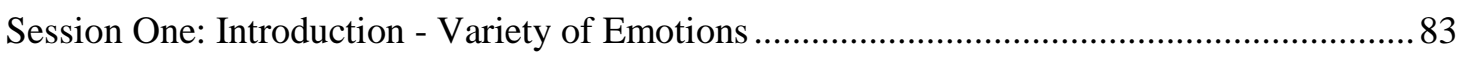

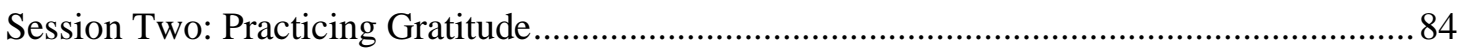

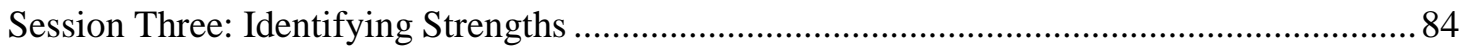

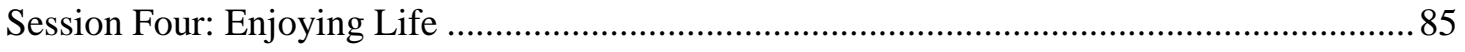

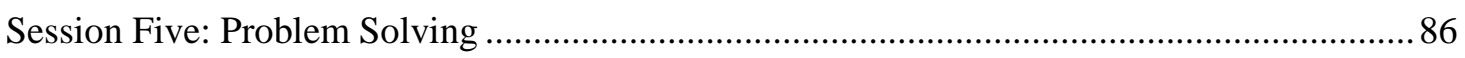

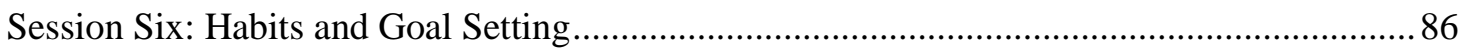

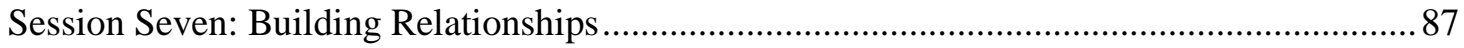

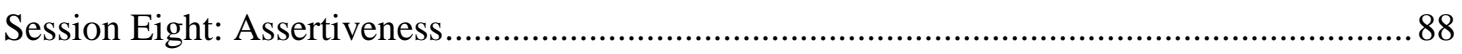

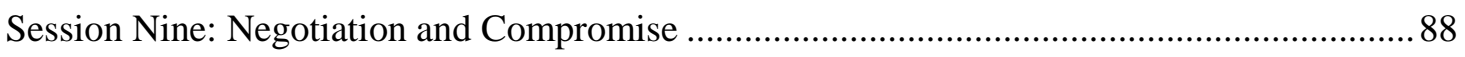

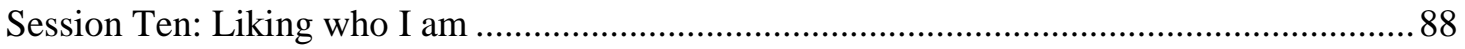

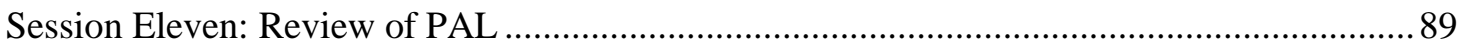

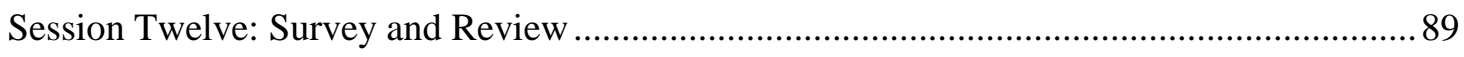

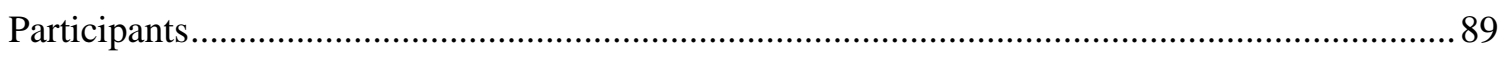

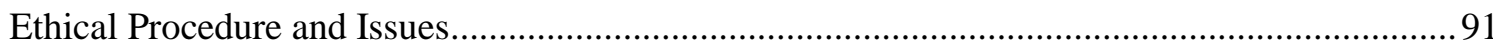

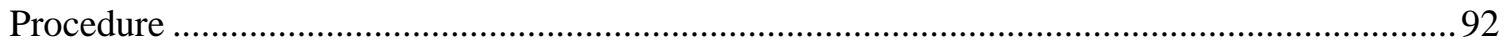


Measures

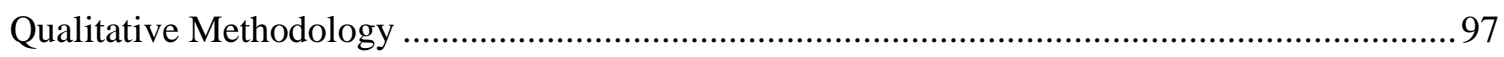

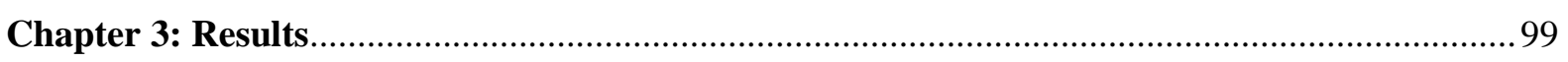

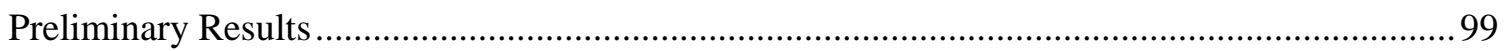

Descriptive Statistics at T1 before Control and Programme Groups were Selected..................99

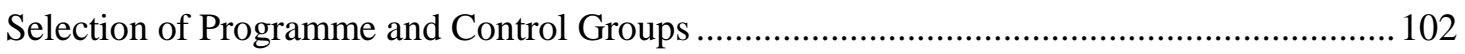

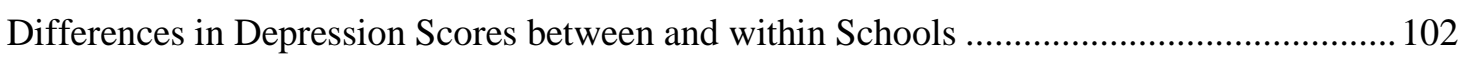

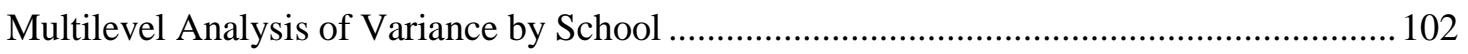

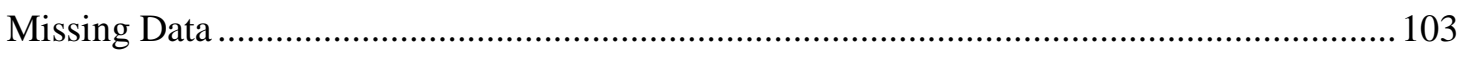

Means and Standard Deviations of the Control Group at all Time Points .............................. 103

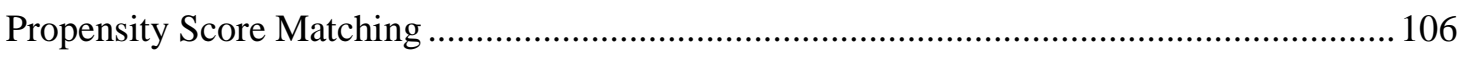

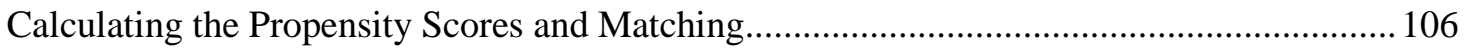

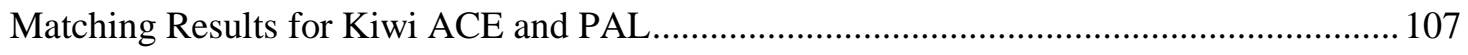

Differences between programme and control groups after programme..................................... 111

Differences between Kiwi ACE and its Matched Control Group......................................... 111

Prediction 1: Kiwi ACE would decrease Depressive Symptoms.............................................. 111

Prediction 2: Kiwi ACE would Decrease Maladaptive Coping ............................................ 111

Prediction 3: Kiwi ACE would increase Adaptive Coping.................................................... 111

Prediction 4: Kiwi ACE would decrease Total Difficulties .................................................... 112

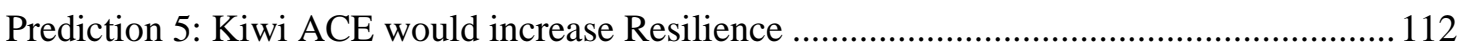

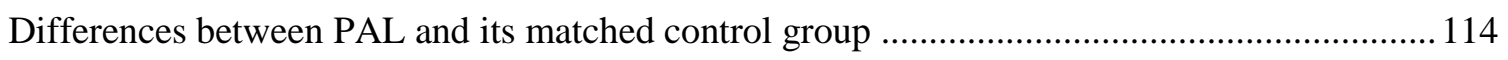

Prediction 8: PAL would increase Psychological Well-being ............................................... 114

Prediction 9: PAL would increase Satisfaction with Life ....................................................... 114

Prediction 10: PAL would increase Subjective Happiness ...................................................... 114

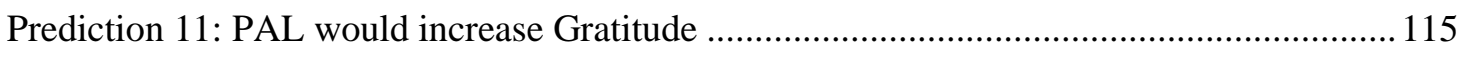

Prediction 12: PAL would increase Personal Resilience ..................................................... 115

Prediction 13: PAL would decrease Depressive Symptoms .................................................. 115

Differences Between the Kiwi ACE and PAL Programmes ................................................... 116 


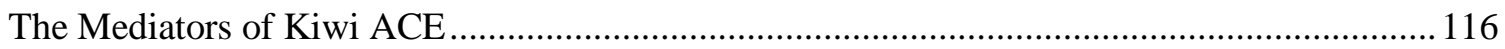

Prediction 6: Total difficulties would function as a mediator between Kiwi ACE and

Depression.

Prediction 7: Maladaptive coping would function as a mediator between Kiwi ACE and

Depression.

Exploratory Mediation

The Mediators of PAL

Prediction 14: Happiness would function as a mediator between PAL and Well-being 120

Prediction 15: Gratitude would function as a mediator between PAL and Well-being.

Exploratory Mediation

Qualitative Results

Kiwi ACE Qualitative Results 130

Why Students Joined the Group.

How students felt about being asked to join Kiwi ACE

How Kiwi ACE participants were treated by their peers for being in the group

Group Dynamics

How well did Kiwi ACE participants get on with their peers and leaders in their group?

Distraction and Participation

Review of Kiwi ACE Sessions in Content and Process

Leaders' Feedback on Sessions.

Comparison of Leaders' and Students' Ratings of Sessions

Overall Assessment of Kiwi ACE by Leaders and Students

How did the students find the programme overall?

Did students continue to practice the skills learnt?

Students' Suggestions of Improvements to the Programme

Strengths, Concerns and Improvements raised by the Leaders of Kiwi ACE

Structure

Student Engagement 
Appropriateness of Programme

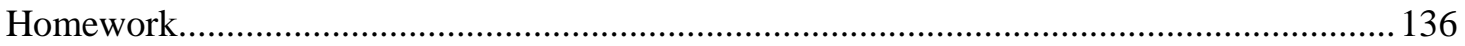

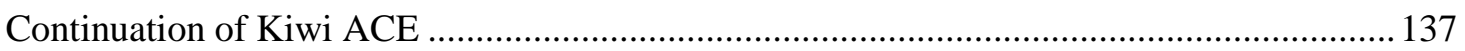

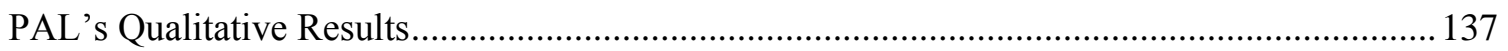

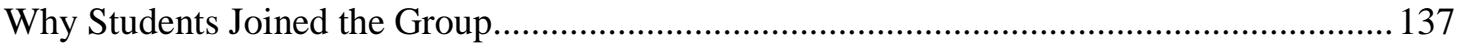

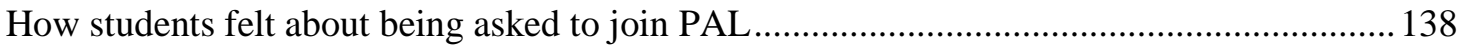

How PAL participants were treated by their peers for being in the group............................... 138

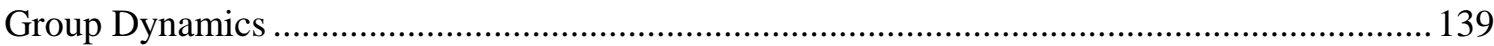

How well did PAL participants get on with their peers and leaders in their group?............... 139

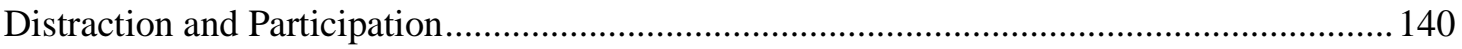

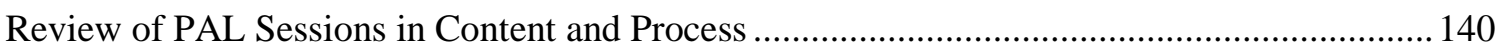

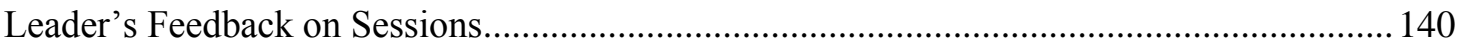

Comparison of Leaders' and Students' Ratings of Sessions .................................................... 142

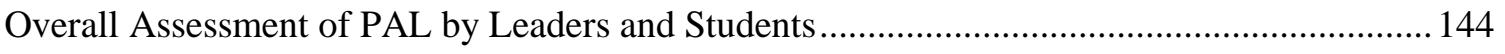

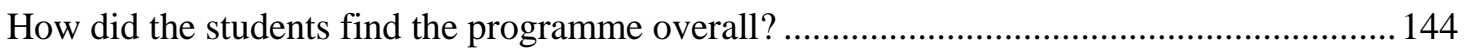

Are students still practicing skills at the end of the 12 week programme? ............................ 144

Students' Suggestions of Improvements to the Programme .................................................. 144

Strengths, Issues and Improvements Raised by the Leaders of PAL ..................................... 145

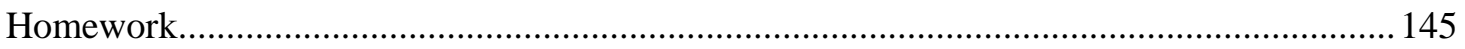

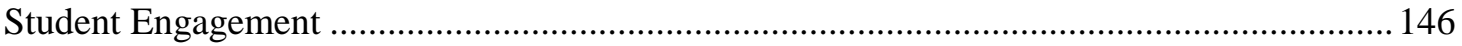

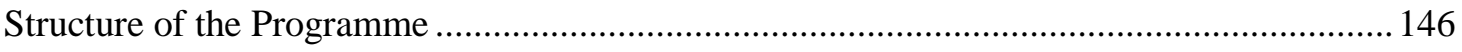

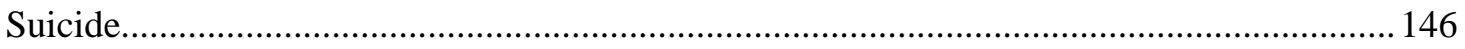

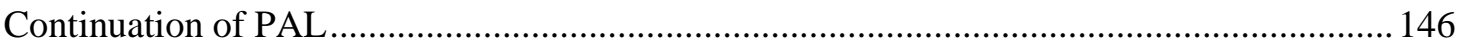

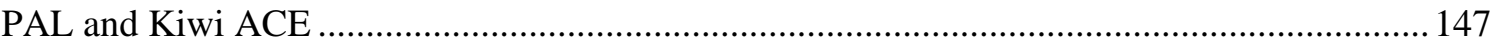

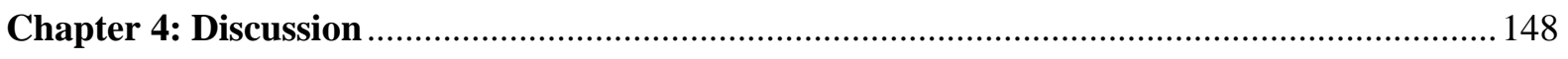

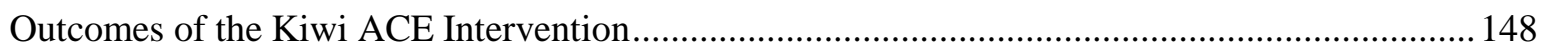

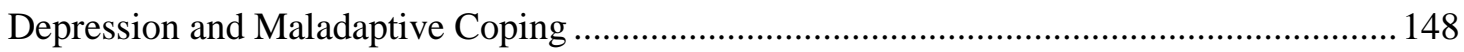

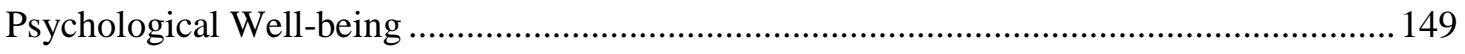


Resilience

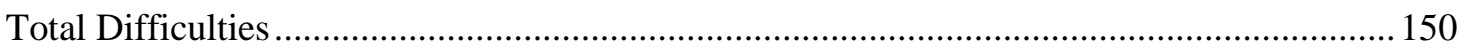

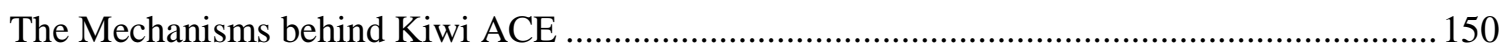

Mediators between Kiwi ACE and Depressive Symptoms.................................................. 150

Mediators between Kiwi ACE and Psychological Well-being ............................................... 151

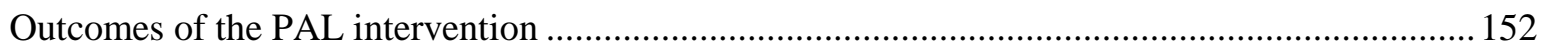

Psychological Well-being, Subjective Happiness, and Satisfaction with Life......................... 152

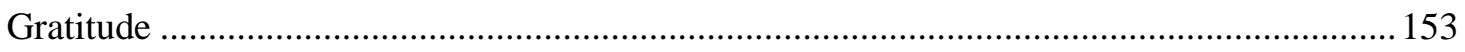

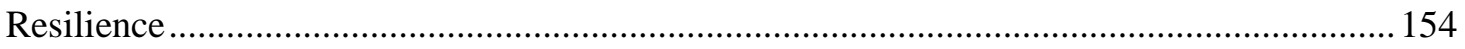

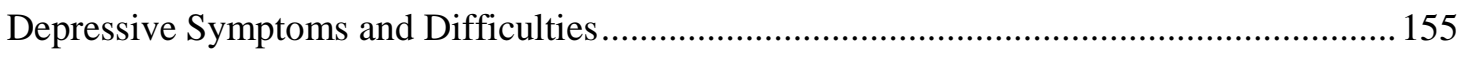

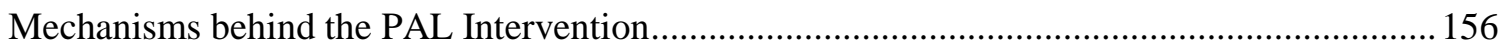

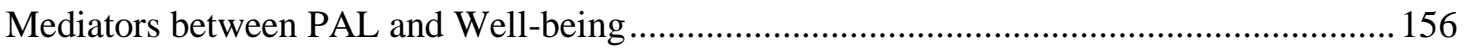

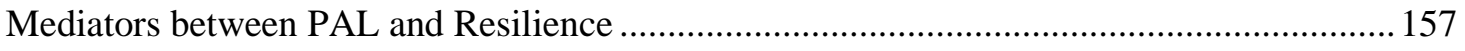

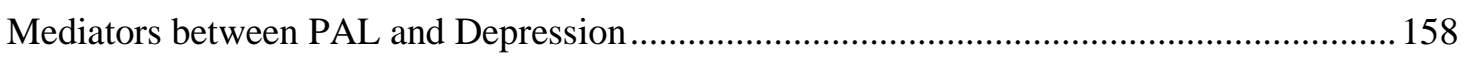

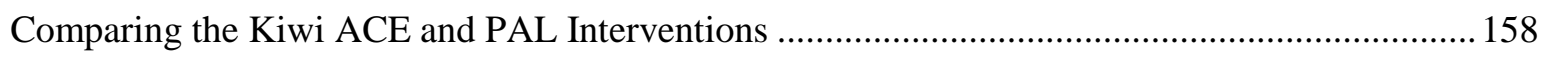

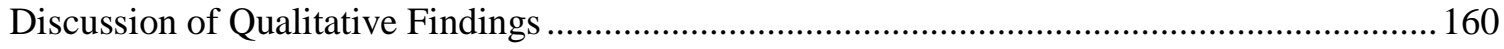

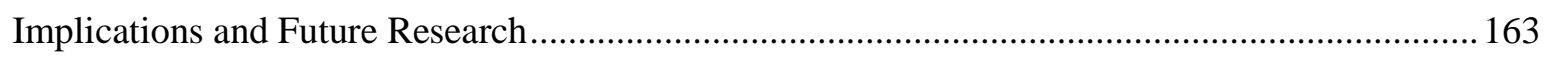

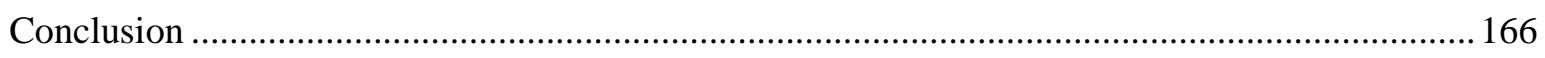

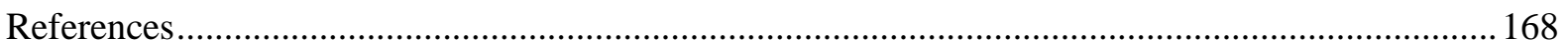

Appendix A: Realistic Thinking Form, from Kiwi ACE Group Book .............................................. 199

Appendix B: Problem Solving Steps from the Kiwi ACE Facilitator's Handbook............................ 200

Appendix C: Problems to solve from the Kiwi ACE Facilitator's Handbook....................................201

Appendix D: Fun Activities Form, from the Kiwi ACE Student Group Book ….............................. 202

Appendix E: Seven Steps of Negotiation, from the Kiwi ACE Facilitator's Handbook .....................203

Appendix F: Negotiation Form, from Kiwi ACE Student Group Book .......................................... 204

Appendix G: Beliefs that Rob and Boost Self-esteem, from Kiwi ACE Group Book ......................205

Appendix H: Steps to Boost Self-esteem, from Kiwi ACE Group Book ……….............................206

Appendix I: Kiwi ACE Idea Sheet, from Kiwi ACE Facilitator's Handbook ....................................207 
Appendix J: Character Strengths, From the PAL Group Book.

Appendix K: Character Strength Survey, from PAL Group Book

Appendix L: Flow Chart, From PAL Group Book

Appendix M: Flow Chart, From PAL Group Book

Appendix N: Savouring Activities, From PAL Group Book

Appendix O: Goal Setting Rules, From PAL Facilitator's Handbook

Appendix P: Goal Setting Action Plan, From PAL Group Book

Appendix Q: Acts of Kindness Ideas, From PAL Group Book

Appendix R: PAL Idea Sheet, From PAL Facilitator's Handbook

Appendix S: Table of comparison between PAL and Kiwi ACE. 


\section{List of Tables}

Table 1: Self reported ethnicities of the control and programme groups. .90

Table 2: Gender, decile, school and year roll numbers and types of schools that participated in each programme.

Table 3: The Scales in the Survey and their Author, Number of Items, Cronbach's Alpha and Purpose in the Study....

Table 4: Correlations of Difficulties, Depression, Resilience, Well-being, Happiness, Gratitude, Satisfaction with Life, Adaptive Coping and Maladaptive Coping

Table 5: Depression score differences between control and programme groups in each school

Table 6: Control group T1-T4 Means and Standard Deviations of Difficulties, Depression, Resilience, Well-being, Gratitude, Happiness, Satisfaction with Life, Adaptive Coping, and Maladaptive Coping .105

Table 7: Control and Kiwi ACE means, standard deviations, $F$ score, significance and effect size in differences at T1 for Difficulties, Depression, Resilience, Well-being, Happiness, Gratitude, Satisfaction with Life, Adaptive Coping and Maladaptive Coping .108

Table 8: Control and PAL means, standard deviations, $F$ score, significance and effect size in differences at T1 for Difficulties, Depression, Resilience, Well-being, Happiness, Gratitude, Satisfaction with Life, Adaptive Coping and Maladaptive Coping 109

Table 9: Comparing number of male, female and ethnicity (NZ European, Māori, Pacific Islander, Asian and other ethnicities) in Kiwi ACE, Matched control and Unmatched Control. 
Table 10: Comparing number of male, female and ethnicity (NZ European,

Māori, Pacific Islander, Asian and other ethnicities) in PAL, matched control and unmatched control

Table 11: Weak effects in Resilience, Happiness, Life Satisfaction and Difficulties when comparing Kiwi ACE with matched control.

Table 12: Standardised indirect effects from mediational relationships between variables in Kiwi ACE 120

Table 13: Standardised indirect effects from mediational relationships between variables in PAL

Table 14. The means of students ratings on how much they enjoyed, found helpful, and practiced each session in Kiwi ACE .134

Table 15 The means of students ratings on how much they enjoyed, found helpful and practiced each session in PAL 


\section{List of Figures}

Figure 1: Standardised coefficients of the total, direct, and indirect effects of group on Well-being mediated by T2 Happiness.

Figure 2: Standardised coefficients of the total, direct, and indirect effects of group on Well-being mediated by T3 Happiness

Figure 3: Standardised coefficients of the total, direct, and indirect effects of group on Well-being mediated by T2 \& T3 Happiness

Figure 4: Standardised coefficients of the total, direct, and indirect effects of group on Well-being mediated by $\mathrm{T} 2$ Gratitude

Figure 5: Standardised coefficients of the total, direct, and indirect effects of group on Well-being mediated by T3 Gratitude.

Figure 6: Standardised coefficients of the total, direct, and indirect effects of group on Well-being mediated by T2 \& T3 Gratitude. .126 


\section{Chapter One: Introduction}

Chapter one includes a brief background of the problem and the aim of the current study. A literature review, divided into two main sections, follows the aim. The first main section provides an overview of the literature on resilience, including its research history and three main differences in the conceptualisation of resilience, that is: approaching resilience as an outcome, a process and as a set of personal characteristics. Resilience interventions are introduced, leading to a section on the importance of building resilience in regards to preventing depression. The section on preventing depression discusses the symptoms and impact of depression in early adolescence to set the scene for further discussion on how prevention programmes based on building positive emotions aim to decrease depressive symptoms. At this point school-based depression prevention programmes are introduced and methods of best practice are discussed. Following this section is a focus on programmes based on the Cognitive-Behavioural Model (CBT), including past trials of ACE (Adolescents Coping with Emotions), and an adapted version called Kiwi ACE which was used in this current study

The second main section examines how positive psychology contributes to an understanding of resilience. The section opens with an introduction to and explanation of positive psychology, including the emphasis of studying mental health and wellbeing not only disorder. Evidence showing the independence of positive and negative mental states is presented. This is followed by a section examining the strong link between high resilience and a high capacity for, and expression of, positive emotion. Fredrickson's (1998; 2001) broaden-and-build theory is introduced. It helps to explain the link between positive emotions and resilience. Also, Fredrickson's theory is central to the development of the Positive Approaches to Life (PAL) programme implemented in this current study. Following Fredrickson's theory is a section dedicated to the explanation of the adaptive and maladaptive roles both positive and negative emotion can have on wellbeing. Emotional regulation strategies are then discussed, as they have been found to be a core component to resilience. The constructs of psychological well-being, happiness and satisfaction with life are defined. Scales that measure both hedonic and eudaimonic concepts of happiness are introduced. Well-being interventions are described and lead to the section on intentional activities that are used in PAL. Each topic in the Positive Approaches to Life programme is introduced and discussed; these topics are gratitude, savouring, kindness, flow, goal setting and character strengths. The gaps in well-being interventions are discussed, leading to a more detailed 
introduction of the current study and its predictions.

The structure of chapter one is framed around the development of research over time in regards to resilience and preventative programmes. The introduction begins with a discussion on resilience, as building resilience is central to this project. The direction of discussion moves from longstanding methods of practice to the field of positive psychology. Positive psychology is not 'new' but it is still a developing field of study and therefore many of the discussions are still evolving. The title can sometimes cause a misunderstanding of what positive psychology is about, it is the present author's opinion that a more appropriate name for this field of study would be the study of psychological health.

\section{Background to the Problem and Aim of this Study}

Currently the majority of programmes aimed at preventing depression in adolescents seek to decrease depressive symptoms but do not look to increase adolescent wellbeing. Given that mental health is not only the absence of psychopathology but is also the presence of psychological well-being (Rashid, 2009), treating an individual's psychopathology is only half of the equation. One could argue that if an individual struggling with mental illness was able to develop more psychological resources, they might gain more effective tools when facing internal and external challenges. The broaden-and-build theory proposes that positive emotions help to build these psychological resources by broadening cognitive pathways and creating more opportunities to do so (Fredrickson, 1998; 2001). Studies show that negative affect and positive affect have two different routes in the brain and are impacted independently (Cacioppo \& Gardner, 1999; Davidson, 1992; LeDoux, 1995; Tomarken, Dichter, Garber, \& Simien, 2004; Tomarkenand \& Anita, 1998). Though traditional programmes do aim to increase adaptive strategies, they are very symptom-specific, and mostly focus on strategies for regulating negative emotions and experiences (e.g., examining unrealistic thinking styles; i.e., Cardemil, Reivich, Beevers, Seligman, \& James, 2007; Lowry-Webster, Barrett, \& Dadds, 2001; Merry, Wild, Bir, \& Cunliffe, 2004). However, research suggests that strategies aimed at regulating positive affect can have widespread repercussions beyond preventing maladaptive behaviour (Folkman \& Moskowitz, 2000) . Using strategies that broaden cognitive pathways is an effective way to regulate positive emotions can help an individual to flourish and become a happier person by finding more pleasure, meaning and engagement in their life. Additionally positive emotions can help build future resilience against the detrimental effects of lingering negative emotions and experiences. 
As of yet, there seems to be no school-based prevention programme that incorporates the different strategies shown to increase wellbeing. Additionally, most programmes neglect to report possible mediators between the programme and the outcome, leaving us in the dark as to how the programme increased wellbeing or decreased depression. One construct that could help explain the mechanisms behind change through intervention, is the measurement and study of personal resilience. Personal resilience is the ability be self-efficient, flexible and resourceful when faced with adversity (Damásio \& Borsa, 2011).The dominant aim of most prevention programmes is essentially to build resilience in adolescents, however, generally programmes do not directly measure resilience because often resilience is viewed only as a process (Masten, 2001). For example, if programme participants show a decrease in depressive symptoms, researchers explain this as an increase in resilience (e.g. LowryWebster et al., 2001). Yet, without direct measurement it is difficult to determine if, and how, resilience was developed in participants.

The present study compared and contrasted two prevention programmes with a focused attention on identifying possible mediators of both programmes. Kiwi ACE focused on decreasing depressive symptoms using CBT strategies shown to work in the past, and PAL sought to increase wellbeing by using a combination of activities shown to increase a capacity for positive emotion. Both of the programmes aim to increase personal resilience by developing students' psychological resources. The current study measured personal resilience and sought to identify effective strategies that contributed to resilience. It was of particular interest to this current study to examine if building a capacity for positive emotions would enhance resilience.

This study sought to confirm and expand literature on psychological health by comparing and contrasting the effects of two prevention programmes, one focused on reducing negative affect and the other focused on enhancing positive affect, and by revealing possible pathways that might lead to increased wellbeing and resilience and reduced negative affect and depressive symptoms. 


\section{Literature Review: Section One}

"It is not the strongest of the species that survives, nor the most intelligent, but the one most responsive to change."-Charles Darwin

\section{Resilience}

Some adolescents develop into positive aspiring young adults despite going through challenging circumstances while others going through similar circumstances constantly struggle with depression, anxiety or lack of self confidence. Individuals able to adapt positively despite adversity have been labelled 'resilient', however, whether this resilient adaptation can be learnt are questions that still need further investigation. When a child is exposed to risk factors such as maltreatment and poverty, he or she will likely be propelled on a trajectory towards poor academic, social and behavioural functioning. It is important to know if there are external factors (such as school-based programmes) that could help change the direction of this path. The study of resilience can inform interventions on what these factors might be.

\section{History of Resilience Research}

Early investigations of patients with disorders were focused primarily on understanding maladaptive behaviour, and the patients who showed relatively adaptive patterns were considered 'atypical' and were given little attention. Norman Garmezy was one of the first researchers to focus on this 'atypical' group and deem their adaptive patterns noteworthy. He was studying the development of children with parents who manifested schizophrenia and found that there was a small but consistent number of children that showed positive outcomes and had little sign of incompetence or pathology (Garmezy, 1971). Garmezy proposed that studying children who developed positively despite having a highrisk status could contribute significantly to advancing theories of etiology in psychopathology and could guide interventions (Garmezy, 1971). This led to an upsurge of studies on 'resilient children', seeking to identify factors that protect against maladaptation by learning what differentiates children who adapt well in high-risk contexts to those that adapt poorly (Garmezy, 1995; Beeghly \& Cicchetti, 1994; Luthar, 1999; Moran \& Eckenrode, 1992, O’Dougherty- Wright, Masten, Northwood, \& Hubbard, 1997, Richters \& Martinez, 1993; Rutter, 1979; Wells \& Schwebel, 1987, Werner \& Smith, 1982, 1992).

In some early writings, children that evidenced resilience were labelled "invulnerable" or "invincible" (Anthony, 1974; Anthony \& Cohler, 1987; Werner, Bierman, 
$\&$ French, 1971). These terms are now considered misleading as they imply psychological invincibility, suggesting that these children will be stress-resistant permanently and regardless of their context. Over the last few decades it has become clear that new vulnerabilities and strengths can emerge with changing life circumstances and environmental input, signifying that resilience is relative, can be influenced and has boundaries (Masten \& Garmezy, 1985; Werner \& Smith, 1982). Masten posits that resilience is a natural outcome of basic human adaptational systems (Masten, 2001). If a child has the resources needed for adaptive healthy development, then in stressful situations the child will be able to have the tools to 'bounce back' relatively quickly after stress and not be negatively impacted in the long-term (Lazarus, 1993). However, if a child's adaptation systems are impaired, then the risk for development problems is much greater and the child will evidence less resilient behaviour and fewer resilient characteristics (Masten, 2001).

The study of resilience has come to recognise that many external and internal factors help to determine resilient functioning. Some of the consistent factors that have been shown to be associated with resilience are: good parent-child relationships, a positive school climate, self esteem and self efficacy in at least one domain of life, planning skills, and a warm, close personal relationship with an adult (Richardson, 2002; Rutter, 1993). Studies have sought to identify how protective factors may contribute to positive outcomes to help understand how resilience might develop and how it potentially could be enhanced (Cowen et al., 1997; Luthar, 1999). Determining the mechanisms behind resilience can help to identify what is needed to change the trajectory of high-risk children and children showing signs of maladaptation in a more positive direction. However, due to the encompassing nature of resilience and the many fields of literature taking an interest in resilience, there are a range of different theories seeking to explain and operationalise resilience. As of yet there is no general agreement on how to best conceptualise resilience. Though what is evident when examining the literature is that resilience has been conceptualised as an outcome (e.g. Vinson, 2002), as a process (e.g. Masten, 2001) and as a set of personal characteristics (e.g. Jacelon, 1997).

\section{Resilience as an Outcome}

Approaching resilience as an outcome involves the examination of effective functioning in young people exposed to risk. For example, socio-economic disadvantages such as poor living conditions have been established as predictors of emotional detachment and stress-related problems (Rutter \& Madge, 1976; Essen \& Wedge, 1978; Robins \& Rutter, 
1990; Duncan \& Brooks-Gunn, 1997; Bynner, Joshi, \& Tsatsas, 2000; Schoon et al., 2002). Therefore children who grow up in poor living conditions but evidence adaptive emotional attachment in relationships and an absence of stress-related problems have been considered to be resilient (Werner \& Smith, 2002). Approaching resilience as an outcome helps to operationalise resilience, as it reveals an individual's successful adaptation despite experiencing adversity. However, this approach can lead to confusion about the concept of resilience; with this view any positive adaptation, functional capacity or social competence can be categorised as resilience. Consequently this approach has been criticised for leading to endless definitions of resilience (Blum, 1998). Therefore, conceptualising resilience in this way helps to identify the many outcomes of resilience, though it fails to capture or measure any processes involved that develop resilience and lead to these outcomes.

\section{Resilience as a Process}

Examining resilience as a process seeks to understand the mechanisms that act to mitigate the impact of risk, and identify developmental processes that young people acquire which help them to successfully adapt. This has lead researchers to label certain mechanisms as protective - i.e. mechanisms that act to improve an individual's responses to adversity, or as risk mechanisms, i.e. those that intensify an individual's reaction to adversity (e.g Rutter, 1987, 1999). For example, studies have shown that avoidance strategies (not thinking about the problem) can prolong stress and can result in depression or increase anxiety (Moulds, Kandris, Starr, \& Wong, 2007). In contrast, problem solving strategies (such as discussing the problem with someone) tends to alleviate stress and lead to confidence in oneself and ability to cope (Dumont \& Provost, 1999). These protective and risk mechanisms have been identified in three main areas: in the individual (e.g. characteristics), in their families (e.g. environment, parental practices) and the societies in which they live (e.g. exposure to crime) (Garmezy, 1991; Werner, 1995). Accordingly, measurement instruments have been developed to assess a young person's range of resilience promoting factors and processes available to them in these different areas (i.e. Hjemdal, Friborg, Stiles, Martinussen, \& Rosenvinge, 2006).

Examining resilience as a process entails a comprehensive view of resilience, it helps to identify processes by which resilience is developed and how these processes lead to positive outcomes. One limit to this approach is the unfeasibility of identifying and measuring all protective factors and processes available to an individual in all areas of their life. The process approach to resilience has multiple factors to consider, as well as the 
interaction between a range of risk and protective factors with varying degrees of impact and the risk settings of the individual at varying points of development. All these varying factors to consider in this approach can convolute the concept of resilience, therefore it is important that the researcher is clear on how specific factors contribute to resilience and how the processes are measured. That said, this approach has helped to expand our understanding of resilience by identifying specific routes to resilient adaptation.

\section{Resilience as a Set of Personal Characteristics}

Common resilient protective factors and processes in individuals has led various researchers to conceptualise and measure resilience as a set of trait-like attributes. Qualitative and quantitative research conducted with resilient individuals (individuals found to recover adaptively from a difficult life event or other adverse conditions) has consistently found a set of common characteristics within these individuals (Garmezy, 1971; Maginness, 2007; Wagnild, 2009). Some of these resilient characteristics include: a strong sense of meaning and purpose (Cottle \& Klineberg, 1974; Penick \& Jepsen, 1992; Rutter, 1989; Seligman, 1990; Stipek, Recchia, \& McClintic, 1992), relatedness and social perceptiveness (Blum, 1998; Constantine, Benard \& Diaz, 1999; Werner \& Smith, 1992), and autonomy and positive sense of self (Constantine,Benard \& Diaz, 1999; Dahlin, \& Cederblad, 1993; Cederblad, 1996). These set of characteristics, among others, have been shown to moderate the negative effects of stress, promote adaptation and help individuals to successfully cope with change or misfortune (Wagnild \& Young, 1993). There are a range of scales that have been designed to measure specific internal protective factors and processes thought to be indicative of resilience. Ego-resilience is one scale that encompasses a set of traits reflecting general resourcefulness and sturdiness of character, and flexibility of functioning in response to varying environmental circumstances (Block, \& Kremen, 1996). Another scale is the Resiliency Scales for Children and Adolescents (RSCA), which are three scales that assess the relative strength of three aspects of personal resiliency: sense of mastery, sense of relatedness, and emotional reactivity (Prince-Embury, 2008).

A review of scales measuring resilience found that the Resilience Scale (RS) by Wagnild and Young (1993) was the most appropriate scale to measure resilience in adolescents (Ahern, Kiehl, Sole, \& Byers, 2006). The meta-analysis indicated that the RS manifested desirable psychometric properties and it was applicable to a variety of age groups, including adolescents. Although the RS was originally developed and tested in a female adult population, it has been used successfully with adolescents in at least 18 published studies and 
its validity and reliability have been consistently good. The RS includes five characteristics which are measured through either a 25 item or a 14 item questionnaire. The five characteristics are: perseverance - the ability to continue through difficulty; equanimity - a balanced perspective that moderates responses to challenges; meaningfulness - something to live for; self-reliance - belief in one's own strength to get through difficult times; and existential aloneness - belief that some experiences must be faced alone (Wagnild, 2009). Each of the subscales identifies an important component of resilience that has been shown to generate positive outcomes and help to buffer the effects of negative experiences. For example the characteristic of meaningfulness has been shown to contribute significantly towards psychological well-being and the prevention of developing depressive symptoms. It is necessary for an individual to have purpose in their lives; to pursue activities that give meaning but also to create meaning in activities. When difficult situations present themselves, the sense of meaning in life can disappear, leaving an individual feeling hopeless or lost, which can quickly lead to depression and other poor outcomes. Those who are able to keep finding meaning in their everyday lives during challenging circumstances can avoid feelings of hopelessnes, despair (e.g. Tennen \& Affleck, 2002) and diminished satisfaction in life (e.g. Schimmack, Oishi, Furr, \& Funder, 2004). The other subscales in the RS show similar pathways leading to wellbeing and avoiding poor outcomes.

Viewing resilience as trait-like has caused some contention in the literature. Various authors caution measuring resilience as a trait because doing so implies that it is stable and unchanging (i.e. Cicchetti \& Rogosh, 1997). Additionally, by measuring resilience as a set of characteristics there is a possibility that only one aspect of resilience will be captured, as there are other areas of resilience (i.e. family, community) and other mechanisms that can promote resilience in an individual (e.g. social support). However, looking at an individual's characteristics does not need to be seen as a contending approach of resilience. The measurement of personal resilience could be used in the context of research that seeks to understand processes that may contribute to positive adaptation and effective coping. There are assessments that have taken on a more multifaceted view of resilience, where personal resilience is measured in combination with social, community and family resources available to an individual (Constantine \& Benard, 2001).

Resilience is seen as the product of adaptive processes that buffer individuals from negative experiences and lead to positive outcomes. Due to the multifaceted construct of resilience there has not always been agreement in the literature about how resilience should be conceptualised or measured. Resilience has been approached in the literature as an 
outcome, a process and as personal characteristics. These approaches do not need to contend with one another, though researchers need to be clear on what it is they are measuring. Personal resilience can be measured with scales that show consistency across populations and age groups. Measuring resilience can inform interventions on if, and how, resilience was enhanced. The following section discusses a resilience-based approach to intervention, what these programmes involve and how they can be effective for at-risk adolescents.

\section{Resilience Interventions}

A resilience-based approach to intervention aims to "build skills and capacities in individuals that will facilitate successful negotiation of high-risk environments" (p.9, Olsson, Bond, Burns, Vella-Brodrick, \& Sawyer, 2003). Though how practitioners go about intervening will depend to some extent of their conceptualisation of resilience and the resources available to them. Predominately there are two types of resilience interventions, those that target risk factors and processes, and those that promote protective factors and processes. Some interventions strive to do both. Targeting protective factors works under the assumption that if enough assets and resources are built or accessible in the life of a child or adolescent, then this will counterbalance the negative effects of adversity (Garmezy, Masten, $\&$ Tellegen, 1984; Masten et al., 1988). Targeting risk factors aims to intervene where assets might be threatened or depleted (Garmezy, Masten, \& Tellegen, 1984; Masten et al., 1988). In childhood, interventions focused on reducing risk have attempted to intervene and reduce exposure to risk, such as, home-based prenatal and antennal care to families at risk of poverty and maltreatment (Hodges \& Blythe, 1992). In adolescence, reducing risk often involves intervening at school. Interventions targeting the stressful transition of starting high-school have been around for some time (Schinke, Schilling \& Snow, 1987). Interventions that focus on increasing assets are those that attempt to increase factors such as self esteem (Quayle, Dziurawiec, Roberts, Kane, \& Ebsworthy, 2001) academic achievement (Weikart \& Schweinhart, 1992) and social skills (Howing, Wodarski, Kurtz, \& Gaudin, 1990).

The Penn Resiliency Program (PRP; Gillham, et al., 2007) defined its aim of building resilience as: "to increase students' ability to handle day to day stressors and to avoid a downward spiral of personal problems". The PRP was developed and implemented in America and has been found to reduce hopelessness, increase optimism, prevent anxiety, depression and behavioural problems. A similar program, the UK Resilience Programme, has been shown to decrease depressive and anxiety symptoms, especially in those students with below average academic performance (Challen, 2009). The content of these programmes 
involve, inviting students to challenge their negative beliefs and encouraging them to come up with alternative interpretations (Cognitive Therapy). Students also learn a variety of techniques for coping and problem-solving, including assertiveness, negotiation, decision making, and relaxation. The aim is for students to apply the cognitive and problem-solving techniques in their lives through group discussions and weekly homework assignments. The Cognitive-Behavioural skills are aimed to mitigate risk by decreasing internal risk factors such as maladaptive coping styles predictive of depression, and to increase protective assets such as social skills. Though these programmes have not always obtained consistent findings (Gillham, Hamilton, Freres, Patton, \& Gallop, 2006; Gillham et al., 2007) the majority of the results suggest these types of interventions are a successful method to increase positive outcomes and decrease risk in children and adolescents.

Most interventions aimed at increasing resilience do not use scales to measure resilience but measure the protective or risk factors involved, such as the programmes above. Often researchers conclude that if the intervention has successfully produced the outcome e.g. mitigated risk by decreasing depressive symptoms, then this has helped to build resilience against depression. Additionally, most scales of resilience measure personal resilience, and interventions do not usually aim to increase personal resilience, because as mentioned above it is often considered more stable, and less likely to be influenced. However personal resilience is still flexible and vulnerable to change (Ahern et al., 2006; Burns \& Anstey, 2010; Neill \& Dias, 2001) and studies have found that personal resilience can be enhanced (e.g. Neill \& Dias, 2001).

An intervention conducted by Neill and Dias (2001) in Australia aimed to build resilience and measure personal resilience with the RS (Wagnild \& Young, 1993). The underlying theory of this intervention was if individuals were faced with a challenge and successfully overcame it, this would enhance their resilience. Forty one participants were involved in a 22-day Outward Bound programme, which involved physical, emotional and social intensity. Thirty one university students were in the control group, they were about the same age as the experimental group (20-24 years of age), and they filled out the RS in their tutorial classes. Baseline RS scores were the same in both groups before the programme. The intervention significantly increased resilience scores in participants directly after the course compared to the control group. This study has limitations. One being that it did not have a matched control group and another was no follow up tests were conducted. However, it suggests that personal resilience can be enhanced. Also their finding suggests that the Resilience Scale, is measuring what it intends to, that is: adapting positively despite 
adversity.

Results from resilience interventions suggest that resilience may be enhanced by reducing risk and increasing protective factors and processes in individuals. Using a resilience-based approach with at-risk adolescents could help to reduce their depressive symptoms, lowering their likelihood of developing depression in the future. The following section discusses the implications of individuals evidencing depressive symptoms at a young age. An outline of the symptoms of depression and how they are measured are presented to set the scene for further discussion on how interventions and strategies aim to decrease depressive symptoms in adolescents.

\section{Building Resilience and Preventing Depression}

Research on resilience has captured the attention of health professionals concerned with the high rate of depression. The devastating impact depression has on the individual goes beyond just the diagnostic symptoms. High levels of depressive symptoms in children and adolescents have been linked to academic problems (Blechman \& Culhane, 1993; Chen, Rubin, \& Li, 1995; deMan \& Leduc, 1995), drug and alcohol abuse (Riggs, Baker, Mikulich, \& Young, 1995), family and social dysfunction, physical ill health and suicide (McGorry, Purcell, Hickie, \& Jorm, 2007; Merry, McDowell, Wild, Bir, \& Cunliffe, 2004). Children and adolescents with subclinical levels of depression also often evidence many of these difficulties (Gillham, 2003; Gotlib, Lewinsohn, \& Seeley, 1995). Research indicates that emotional difficulties in adolescence prevents individuals from obtaining optimal resources in adulthood and can affect all areas of their life including their future relationships, occupation and finances (Bayer \& Sanson, 2003; Cicchetti \& Toth, 1998).

In New Zealand approximately $15 \%$ of females and $7 \%$ of males experience a major depressive episode by the age of 18 years (Adolescent Health Research Group, 2008). Since first onset usually occurs between the ages of 15-19 years, intervention in early adolescence is key. Additionally adolescents who evidence elevated depressive symptoms are more at risk of developing depression later in life. Symptoms of depression in adolescents include: persistent sad or irritable mood, loss of interest in activities once enjoyed, substantial change in appetite or body weight, oversleeping or difficulty sleeping, psychomotor agitation or retardation, loss of energy, feelings of worthlessness or inappropriate guilt, difficulty concentrating and recurrent thoughts of death or suicide. For an adolescent to be diagnosed with clinical depression, five or more of the above-listed DSM (Diagnostic and Statistical Manual of Mental Disorders) symptoms, including at least one of the first two, must persist 
for two weeks or more, and must cause significant distress or functional impairment (American Psychiatric Association, 2000). However, symptoms are often more unstable in adolescents and some days of normal mood within a given two week period does not negate the diagnosis. A range of scales have been designed to measure depressive symptoms in adolescents; one of the most commonly used scales is the Children's Depression Inventory (CDI; Kovacs, 2008) which is frequently used in both child and adolescent populations. The full measure of the CDI has 27 items that measure five components of depression. These components are: Anhedonia, inability or decreased ability to experience joy; Negative selfesteem, belief that you are not good at anything; Ineffectiveness, lack of motivation or inability to complete tasks; Interpersonal problems, difficulty making and keeping close relationships; and Negative mood, irritability, sadness, or anger.

Depression is a debilitating and chronic disease that can start to develop in childhood or adolescence. If symptoms are detected early, then there is more of a possibility to change its course. One of the ways to intervene is to help young individuals build internal and external resources. Accordingly, resilience research has been used to help determine what type of resources are necessary to prevent the eventual manifestation of severe forms of depression. Prevention programmes and resilience programmes are concepts that are often used interchangeably, as they both seek to build resources in order to prevent negative outcomes. The next section discusses depression prevention programmes in schools, how they operate and effective methods to help ensure the validity of the programme and the measurement of true intervention effects.

\section{School-based Preventative Programmes: Universal, Selective and Indicated}

Prevention programmes originated from the idea that depression can be prevented if an appropriate intervention occurs early enough in an individual's life. Most prevention programmes are school-based because they are often the most time- and resource-efficient alternative (Barrett \& Pahl, 2006; Masia-Warner, Nangle, \& Hansen, 2006). A mental health screening in a school can identify students who need support but have gone undetected in the past (Ginsburg \& Drake, 2002). Once 'at-risk' individuals are identified, they can be recruited for an intervention that hopefully changes the negative trajectory of these young people. The benefits of the programme often extend to various settings such as the classroom, peer relations, and home-life. Conduct problems and inattention are common behavioural expressions of depression in adolescence and these behavioural tendencies can disrupt their learning and make social interactions challenging (Lewinsohn, Clarke, Hops, \& Andrews, 
1990). School-based programmes can help students to experience the school environment as a place where their emotional, academic, and social needs are met and their wellbeing is supported and encouraged (Wassef, Ingham, Collins, \& Mason, 1995).

Three main types of prevention programs utilised in schools to prevent depressive symptoms have been defined: universal, selective, and indicated (Mrazek \& Haggerty, 1994). Universal programs are usually designed to enhance general well-being and/or build resiliency and include all students, regardless of their emotional and mental health (Barrett \& Turner, 2004). To establish true preventative effects, students who suffer from depression are often excluded from universal programs. Selective programs include students who share risk factors associated with depression such as parental divorce; and indicated programs are comprised of students with mild or early symptoms of depression, i.e. are considered 'at-risk' (Mrazek \& Haggerty, 1994). Out of the three types, universal programs are the most commonly used in schools, and this has been attributed to the reduced stigma and time associated with them and their broad application (Horowitz, Garber, Ciesla, Young, \& Mufson, 2007). However, research suggests that indicated programs are more effective than universal programs because they target those with elevated symptoms and there is greater chance for improvement (Offord, Kraemer, Kazdin, Jensen, \& Harrington, 1998; Reivich, Gillham, Chaplin, \& Seligman, 2005).

A recent meta-analysis showed mixed results in regards to the effectiveness of schoolbased early intervention and prevention programs for depression (Calear \& Christensen, 2010). Only $50 \%$ of the 42 programs were effective at reducing depressive symptoms. However, indicated programs were the most effective and had the most consistent outcomes at post-test and follow-up compared to selective and universal programs. Other determinants of effectiveness were identified: the programme leader, the control condition, and the length of the programme. Programs that used mental health workers or programme developers, rather than school teachers or counsellors, were more successful. On the second point, the meta-analysis found that programs using attention controls yielded smaller mean effects, suggesting that having other types of controls could inflate intervention effects. Finally, the trials that involved a program that lasted from eight to twelve weeks were more successful at lowering depression than trials that lasted less than eight weeks or longer than 12 weeks. This finding indicates that programs that are too long or too short can be less successful. The authors suggested that if a programme is too short students may not have enough exposure to key ideas or experiences, and if it is too long there may be too much information to absorb. The analysis also revealed that for many of the programs, significant changes were only 
detected at follow-up and did not have immediate significant post-test results. This finding signals the importance of long-term follow-up measurement, for without it a program's effectiveness can be underestimated and the true effect never identified. For preventative effects to emerge it is important to follow the participant post-intervention through a period of possible elevated psychological risk (Greenberg, Domitrovich, \& Bumbarger, 2001).

School-based prevention programmes can be an effective means to help protect adolescents from developing depression later in life. Although the research results are varied, it appears that indicated programmes are shown to be most effective at measuring change as they target individuals with elevated symptoms. Research shows the importance of trained leaders running prevention programmes and ensuring that the control groups are controlling for the difference in the programme techniques. Also, it is imperative that the prevention program is of an appropriate length and longitudinal follow up tests are conducted for accurate assessment.

The present study sought to incorporate all the characteristics mentioned above into the research design, aiming to create programmes that use best-practice methods and that are able to detect intervention effects. The following section looks at specific school-based interventions, namely those that use a Cognitive-Behavioural framework. CognitiveBehavioural strategies and methods are discussed in order to explain the theoretical underpinnings to Kiwi ACE, one of the programmes used in the current study aiming to decrease depressive symptoms of at-risk adolescents.

\section{Cognitive-Behaviour Models of Prevention Programmes}

The majority of prevention programmes use a Cognitive-Behaviour Therapy (CBT) model, as CBT has a longstanding evidence base in the treatment of depression (CBT; Andrews \& Wilkinson, 2002). CBT is not a single therapy but rather a mixed array of techniques grouped under common principles that guide CBT (Craske, 2010). The underlying theory behind this model is that cognitions influence emotions, therefore changing unhelpful thought patterns can help to change negative moods and feelings (and as a result change behaviour). The behavioural component (the ' $\mathrm{B}$ ' in $\mathrm{CBT}$ ) refers to behavioural interventions that are used to challenge the maladaptive beliefs and thought patterns that can trap individuals in a downward spiral of depressive thoughts, cognitions, and behaviours. An example of a behavioural intervention would be encouraging a client to ring a friend if they have not socialised for a long time.

Prevention programmes that use CBT aim to decrease depressive symptoms by 
targeting the negative automatic thoughts and maladaptive beliefs, and coping strategies that are common in adolescents who suffer from depression (Andrews \& Wilkinson, 2002; Spirito, Esposito-Smythers, Wolff, \& Uhl, 2011). A similar structure is used in most CBTbased prevention programmes. Often they begin with helping students identify different emotions and their intensity, then to differentiate thoughts from emotions, and to clarify the links that exist between them. Many of the activities are based on the process of finding evidence for and against unhelpful thoughts and generating alternative and realistic thoughts in order to change negative emotions. Often CBT programmes incorporate strategies aimed to develop problem solving skills, social skills and goal setting, as they work well alongside CBT by providing opportunities for students to practice the CBT skills they are learning.

Some examples of school-based CBT prevention programmes were discussed earlier such as the Penn Resiliency Program (PRP; Gillham, et al., 2007) and the UK Resilience Programme (Challen, 2009), both were shown to decrease depressive symptoms. Other examples are: the Resourceful Adolescent Program (RAP) which was designed in Australia, it incorporates cognitive-behavioural and interpersonal therapy principles and has been shown to be effective in decreasing depressive symptoms post intervention (Shochet et al., 2001). The RAP-Kiwi was adapted for New Zealand, and was found to decrease depressive symptoms up to 18 months after the programme ended (Merry et al., 2004). Another programme trialled in New Zealand that is based on CBT is the Kiwi ACE programme (Adolescents Coping with Emotions) (Woods, 2008). Kiwi ACE is an adaptation of the ACE programme which was originally developed in Australia. The ACE programme deployed in Australia was found to reduce depressive symptoms and increase coping skills for 82 female participants aged between 13-16 years (Kowalenko et al., 2005). Additionally after the programme, participants were more likely to be engaged in productive coping strategies (i.e. problem solving, talking to others) and less likely to be engaged in non-productive coping strategies (i.e. worrying, ignoring the problem, smoking or drinking) compared to those in the control group. Kiwi ACE was adapted to fit the New Zealand context by using culturally relevant language and pictures. When Kiwi ACE was trialled in New Zealand in 2006, it was found to decrease depression up to one year after the programme ended in a sample of 13year-olds, including Māori and Pacific Island students (Woods, 2008; Woods \& Jose, 2011).

Cognitive Behaviour Therapy has a long standing history in efficacy of treating depression. Furthermore prevention programmes based on the CBT model have been shown to be effective at decreasing depressive symptoms in adolescents with elevated symptoms. However, recently practitioners and researchers are asking if is it enough to only aim to 
decrease depressive symptoms. Section two below looks at how promoting wellbeing in atrisk adolescents may help to bring about positive outcomes that could lead to widespread affects rather than only decreasing negative outcomes.

\section{Literature Review: Section Two}

"Mirth and merriment . . bars a thousand harms and lengthens life"' (Shakespeare).

\section{Positive Psychology}

Positive psychology is the study of healthy human functioning, including the characteristics, emotions, cognitions and behaviours that contribute to an individual's present happiness, personal growth, meaning and long-term wellbeing (Snyder \& Lopez, 2005). Positive psychology challenges prevention programmes that are focused purely on correcting a disorder, i.e. focusing on the pathology or what is 'wrong' with an individual. Researchers in the field of positive psychology assert that practitioners should not only aim to mitigate dysfunction but also aim to encourage positive adaptation and increase wellbeing (Linley, Joseph, Harrington, \& Wood, 2006). For a complete picture of human functioning we need to understand the factors that contribute to mental health. Yet psychology is still is in its infancy when it comes to the study of wellbeing; the majority of the literature has studied maladaptive behaviour and mental illness (for a review see: Wood \& Tarrier, 2010). This unbalanced focus originates in the history of psychology which is founded in the dysfunctional behaviour of humans (Seligman \& Csikszentmihalyi, 2000). This focus in the past was necessary, however, as a result the functional healthy side of human psychology has remained relatively unstudied. Only recently, wellbeing, happiness, and positive characteristics and emotions have been given closer attention.

Positive psychology is not new, in that, it is not the first area of study to look into the positive aspects of functioning, but it has brought attention to the deficit of research in this area (Wood \& Tarrier, 2010). Additionally, research in positive psychology has alerted clinicians to certain factors may have been missed in the study and treatment of pathology (Ingram, 2006; Layous, Chancellor, Lyubomirsky, Wang, \& Doraiswamy, 2011; Rashid, 2009). For example, it is possible that the presence of particular strengths in individuals can moderate or mediate dysfunction or risk and an absence of these strengths may cause dysfunction to grow (Gillham et al., 2011; Rashid \& Ostermann, 2009). Furthermore, there is an accumulating range of evidence that shows positive mental states are more than just the 
absence of disorder or distress (Diener \& Emmons, 1984; Lazarus, 2000; Huppert \& Whittington, 2003; Huta \& Hawley, 2008; Watson, 2000).

Programmes that seek to alleviate depressive symptoms rarely if ever assess changes in happiness. However, if an individual receives treatment for depression and is no longer depressed, this change does not necessarily lead someone to becoming a happy, optimally functioning individual (Duckworth, Steen, \& Seligman, 2005). According to Duckworth et al. (2005) reducing depression, anxiety, anger and distress in clients does not automatically result in happiness, as the skills of finding pleasure and engagement are different but could accompany the skills of fighting depression. Sadness and happiness are not opposite endpoints on a single continuum, just as personal strengths and vulnerabilities are not exact opposites (Huta \& Hawley, 2008). Positive and negative affect, although moderately and significantly (negatively) correlated, have been found to have different correlates and show some independence from each other (Bradburn, 1969; Diener \& Emmons, 1984; Watson, 2000; for a competing view see: Russell \& Carroll, 1999). Additionally, neurological studies show that positive and negative affect are associated with different neural structures and are impacted somewhat independently (Cacioppo \& Gardner, 1999; Davidson, 1992; LeDoux, 1995; Tomarken et al., 2004; Tomarkenand \& Anita, 1998).

Huppert and Whittington (2003) investigated the determinants and characteristics of positive and negative wellbeing. They surveyed an adult sample of 9,003 adults with an adapted version of the self-rated General Health Questionnaire (Goldberg,1972) that rated symptoms of psychological distress and well-being. The authors found that over one third of their sample obtained scores that were high on both constructs or low on both, rather than showing a pattern of what one might expect (i.e. low on positive wellbeing and high psychological distress or the other way around). Their finding indicates a degree of independence between positive and negative wellbeing. Additionally, different variables determined the different constructs, indicating that there are different routes to both wellbeing and depression. Physical health problems and lack of social support increased symptoms of depression, whereas these variables had less influence on positive emotions. Furthermore, unemployment negatively predicted wellbeing yet had little impact on depression. Huppert and Whittington conclude that when assessing an individual's quality of life it is essential to include wellbeing measures with measures of dysfunction or distress, or there is the danger of reaching incorrect conclusions about the impact of treatment. These findings also confirm the importance of targeting the different determinants of both wellbeing and depressive symptoms in the treatment and prevention of depression. 
In another study Huta and Hawley (2008) investigated the relationship between psychological strengths and vulnerabilities and how they related to wellbeing in a nonclinical sample, and a clinically depressed sample before and after treatment. Strengths were measured with the Values in Action Inventory of Strengths; a measure of strengths shown to predict well-being (i.e. hope, appreciation for beauty, gratitude) (Seligman \& Peterson, 2001). Cognitive vulnerabilities were measured with the Dysfunctional Attitudes Scale designed to assess cognitive vulnerabilities that have been shown to predispose individuals to depression (such as the need for approval: a tendency to place excessive importance on other people's judgements) (Weissman and Beck, 1978). The results showed moderate correlations between strengths and vulnerabilities, suggesting some independence between the two constructs. Strengths before treatment predicted recovery from depression, whereas cognitive vulnerabilities did not. Strengths were also shown to weaken the relationship between vulnerabilities and well-being. These findings indicate that participants' with higher levels of strengths were better able to benefit from treatment, suggesting strengths may be associated with more favourable prognosis; however participants' strengths did not clearly protect individuals from depression in the first instance.

Consequently positive psychology argues that, firstly, depression and wellbeing must be studied jointly in order to garner a more complete understanding of the functioning of an individual. Secondly, the aim of prevention programmes should not only be to prevent adolescents from developing depression but also to increase their positive psychological resources in an effort to enhance resistance against the effects of stressful events that will occur in the future. The aim is to restore, nurture and build resources in order to deal with life's challenges. Prevention and resilience research fits quite naturally with the positive psychology viewpoint, in that they both seek to enhance protective factors in the individual in order to foster positive outcomes and resistance against negative outcomes. This balanced approach is consistent with the findings that have come from research on resilience and wellbeing. Namely, studies have found that positive emotions help to build psychological resources, mitigate the effects of stress, and help the recovery after trauma. Some examples of this research are given in the section below.

\section{Positive Psychology's Contribution to Resilience}

A recent longitudinal study found that positive emotions predicted an increase in resilience and life satisfaction, and that positive emotions mediated the increase in resilience but life satisfaction did not (Cohn, Fredrickson, Brown, Mikels, \& Conway, 2009). Cohn et 
al.'s (2009) findings suggest that it is the in the moment positive emotions that build resilience, not necessarily having satisfaction in life. They also found that resilience mediated the relationship between positive emotions and increased life satisfaction. This finding indicates that happy people become more satisfied in life by building resources, not just because they feel good. In line with this study, other research has found that individuals high in resilience experience more positive emotions when faced with a life stressor than their less resilient peers (Ong, Bergeman, Bisconti, \& Wallace, 2006; Tugade \& Fredrickson, 2004).

A study using data from before and after the September 11 attacks in America, found that people with pre-existing high resilience were more likely to find positive meaning within the problems they faced. Those high in resilience experienced fewer depressive symptoms and more positive emotions following the attacks (Fredrickson, Tugade, Waugh, \& Larkin, 2003). Positive emotions experienced after the attacks could fully account for the relationship between resilience before the attacks and depressive symptoms after the attacks. That is, positive emotions were found to be the significant factor that protected people against depression. Some of the positive emotions experienced were: gratitude, loving-kindness, and newfound love for friends and family. These findings suggest that positive emotions do not disappear in the face of trauma and distress, and cultivating these positive emotions seems to be key in helping people recover from trauma and to protect them against developing prolonged emotional difficulties.

Folkman and Moskowitz $(2000,2004)$ propose that positive emotions help to act as a buffer against the adverse physical and psychological responses caused by stress. For example positive affect may protect individuals from maladaptive neuroendocrine responses that occur after stressful events that can lead to disease (Epel, McEwen, \& Ickovics, 1998; McEwen, 1998). They also suggest that positive emotions may interrupt the ruminative process that leads to significant mood disturbance (Folkman \& Moskowitz, 2000). In three separate lab experiments, Fredrickson and colleagues tested whether positive emotions would help to speed up the recovery time immediately after negative emotional arousal (Fredrickson \& Levenson, 1998; Fredrickson, Mancuso, Branigan, \& Tugade, 2000). For each investigation, negative emotional arousal was induced, and then participants were randomly assigned to view an emotionally evocative film that induced joy, contentment, neutrality, or sadness. They found that participants in both positive emotion conditions recovered faster from negative emotional arousal (i.e. cardiovascular recovery) than the participants in the neutral or sad conditions. The authors labelled this affect the 'undoing affect', inferring that positive emotions help to undo the lingering effects of negative emotions. 


\section{Fredrickson's Broaden and Build Theory}

Fredrickson's broaden-and-build theory (Fredrickson, 1998; 2001) helps to explain why positive emotions may lead to resilience, future success and enhanced wellbeing. Her theory proposes that positive emotions expand cognitive pathways, which encourages an individual to consider a broader range of options that over time build helpful and adaptive resources. As a result, positive emotions can open up a range of new possibilities in both thought and action. For example, enjoyment can encourage play, which increases exploration, which in turn builds new ideas, and which in turn builds new resources (Boulton \& Smith, 1992). Simple curiosity can lead to in depth knowledge; interest in another person can lead to a long-term fulfilling relationship. Fredrickson explains that the momentary benefits of cognitive broadening and physiological undoing of negative emotions happen while people experience positive affect. Over time these momentary benefits can build up skills and resources, such as the development of effective coping strategies.

Research supports the notion that positive emotions broaden thought-action repertoires. Emotions, such as hopefulness and enjoyment, have been shown to lead to an expansion of attentional focus (Derryberry \& Tucker, 1994), broaden the scope of cognition and action (Isen, 1987; Isen, Daubman \& Nowicki, 1987, Kahn \& Isen, 1993), promote creativity and flexibility in problem solving (Isen, et al., 1987; Isen \& Geva, 1987; Isen, Johnson, Mertz, \& Robinson, 1985), and build intellectual resources (Bryan, Mathur, \& Sullivan, 1996). Additionally positive emotions have been shown to produce wider visual search patterns, novel and creative thoughts and actions, create more inclusive social groups, and more flexible goals and mindsets (for reviews see: Ashby, Isen, \& Turken, 1999; Fredrickson \& Cohn, 2008). A growing empirical basis is being generated for the broadenand build theory as studies consistently find that positive emotions promote physical, emotional, and mental health (Fredrickson \& Losada, 2005; Tugade, Fredrickson, \& Barrett, 2004; Fredrickson, Mancuso, Branigan, \& Tugade, 2000).

Therefore positive emotions have a functional role beyond just making us feel good. Research suggests that positive emotions operate as internal protective and repair mechanisms that help us to function despite negative events and emotions, to be able to continue through challenges, work through difficulty and heal from past hurt. Not surprisingly having a capacity for positive emotions seems to be key to resilience. To give some balance to the discussion on the role of emotions; the role of negative emotions and the maladaptive consequences of both negative and positive affect is discussed in the following section. This discussion then leads to the importance of emotional regulation. 


\section{Positive and Negative Emotions}

Negative emotions also have an adaptive role, though in contrast to positive emotions, negative emotions usually narrow attention, cognition and physiology in response to an immediate threat or problem (Carver, 2003; Cosmides \& Tooby, 2000). For example, fear is usually produced in response to a threatening situation. Fear precipitates in an individual a flight-or-fight response. The body releases a quick physiological response (i.e. adrenalin) in order to have the maximum capacity to choose and enact the best option among the narrow options available. This fear response has been adaptive to human survival, helping many in the past to survive close contact with natural predators. In a different way, sadness can also have an adaptive role in that it signals to the individual that something is wrong and can bring about motivation for change or elicit support from others (Davidson, 1988). Negative emotions can also have a broader adaptive role, for example anger, which can rise out of a sense of injustice, can prompt an individual to fight for social justice. Also anger can help individuals to achieve their goal and overcome obstacles (Davidson, 1988). In sum, negative emotions, like positive emotions, also play a critical role in our wellbeing.

However, if negative emotions linger for longer than for their initial adaptive role, or keep appearing when there is an overestimation of threat and a underestimation of the ability to cope; they can cause significant emotional, psychological and health problems. For example, a general sensitivity to negative emotions is related to anxiety and depression (Clark $\&$ Watson, 1991). Individuals who suffer from depression are likely to display longer durations of negative emotion, are less able to recover from emotional upset (NolenHoeksema, 1991) and experience difficulty in generating or maintaining positive expression (Bijttebier, Raes, Vasey, \& Feldman, 2012). On the flip side, positive emotions can also lead to maladaptive outcomes, such as the overindulgence in, or addiction to, the pleasure derived from some activities. The dopamine reward system is triggered in the brain as a result from pleasurable stimulants, and can contribute to the development of addictions and result in maladaptive behaviour (e.g. Hyman, Malenka, \& Nestler, 2006; Xiao et al., 2009). Some examples include sexual addiction, over-eating, over-spending, gambling addiction, and substance abuse. Additionally there are times when positive emotions may not be beneficial, depending on the context. For example, McNulty and Fincham (2012) discuss how forgiveness and optimism can be harmful in the context of an abusive relationship.

Despite the fact that both negative and positive emotions can play an adaptive role in functioning, it is also true that both can lead to maladaptive behaviour. These maladaptive and adaptive behavioural outcomes reveal that labelling emotions simply as good or bad is 
misleading. Also, the position take here is that there is more to adaptive functioning and resilience than merely experiencing less negative emotion and more positive emotion.

\section{Emotional Regulation}

The key to adaptive functioning, then, is not just what emotion an individual experiences, but the way in which an individual regulates their emotional response. One of the main characteristics of psychopathology is maladaptive emotional regulation (Gross, Richards, \& John, 2006; Gratz \& Roemer 2004; Rottenberg and Gross 2003). Likewise, one of the main characteristics of resilience is the capacity to use effective emotional regulation strategies (Davidson, 2000; Tugade \& Fredrickson, 2007). Emotional regulation is an individual's capacity to elicit, maintain and modulate emotions, including having a sense of control about how these emotions are experienced and expressed (Gross et al. 2006, Thompson, 1994). Regulating emotions involves taking action to alter the intensity of an emotion and using strategies to extend or shorten the emotional experience (Larsen \& Prizmic, 2004). Not all strategies used for emotional regulation are equally effective (John \& Gross, 2004). Studies show that individuals who suppress, ruminate on, or avoid negative emotions are more likely to develop depressive symptoms (John \& Gross, 2004). Also, using strategies to down-play negative emotions tends to be less effective than using strategies to elicit positive emotions when experiencing stress (Feng et al., 2009; Folkman \& Moskowitz, 2000).

Research suggests that a key mechanism underlying resilience is having the ability to regulate positive emotions in order to cope with negative experiences and emotions (Davidson, 2000; Tugade \& Fredrickson, 2007). Highly resilient individuals often use coping strategies that elicit positive emotions to regulate negative emotional situations (Folkman \& Moskowitz, 2000; Tugade \& Fredrickson, 2007). Some examples are: engaging in relaxation activities during heightened stress to allow time to interpret and assess problems with a different mindset (Grossman, Niemann, Schmidt, \& Walach, 2004; Kabat-Zinn, 1990; Smith, 1990); allowing exploration of thought to consider behavioural alternatives (Werner and Smith, 1992); and infusing meaning into ordinary daily events which can help to neutralise the negative situation and help generate gratefulness and optimism (Folkman \& Moskowitz, 2000). According to Bargh and Chartrand (1999), it is possible that positive emotional responses in individuals high in resilience have become an automatic coping strategy due to repetition. Once the coping strategy has become automatic, it frees up cognitive resources, which contributes further to their ability to recover from negative events 
(Isen and Diamond, 1989). It has also been suggested that positive affect has the ability to change the wiring of the circuitry that underlies emotional regulation (specifically in the prefrontal cortex and amygdala), helping to develop resistance against psychopathology and other maladaptive psychological states (Davidson, 2000).

A core part of resilience is the ability to effectively use strategies to regulate emotions. These strategies can help to protect individuals from the full impact of negative experiences and promote happiness and wellbeing. Consequently activities designed to build resilience may promote wellbeing and happiness. Likewise activities designed to increase happiness and promote wellbeing will also build resilience. Before expanding on effective strategies that promote positive outcomes, it is necessary to define and distinguish between the concepts of happiness, satisfaction with life and psychological well-being, which are discussed in the following section.

\section{Happiness, Satisfaction with Life and Psychological Well-being}

Philosophers have attempted to define happiness and the pathways leading to happiness for many centuries. Discussions on happiness can be traced back to writings by both Aristippus and Aristotle who had contrasting views. Aristippus (435-366 BCE) defined happiness as the maximisation of pleasure and minimisation of pain - this was termed hedonic happiness (Watson, 1895). In contrast, Aristotle (384-322 BCE) stated that happiness was more than seeking pleasure but hinged on being true to yourself by identifying your virtues, cultivating them, and living in accordance to them - this is termed eudaimonic happiness (Hughes, 2001). Interestingly these definitions are still relevant today and researchers have designed scales in order to measure both hedonic and eudaimonic happiness, these are summarised below.

\section{Hedonic Happiness}

The hedonic type of happiness is often labelled subjective happiness or subjective wellbeing. The literature defines subjective happiness as consisting of two components: a) positive vitality - the frequent experience of positive affect, and infrequent experience of negative affect, and b) satisfaction with life - a positive cognitive evaluation of one's own life (Diener \& Biswas-Diener, 2008; Snyder \& Lopez, 2005). In other words subjective happiness is thinking and feeling positively about your life. Measuring subjective happiness can be measured by assessing the frequency and intensity of positive and negative affect in addition to asking individuals to rate their overall satisfaction with their life. Studies have shown that affect is best measured at regular intervals, where the participant records what she is feeling 
when she is feeling it (e.g. the Positive and Negative Affects Scales (PANAS); Watson \& Clark, 1988). The Satisfaction with Life Scale (SWLS) (Diener, Emmons, Larsen, \& Griffin, 1985) has been shown to be a reliable measure of the self evaluation component of happiness (Pavot \& Diener, 2008). The SWLS is constituted by five items, including: in most ways I am satisfied with my life and the conditions of my life are excellent. Sometimes life satisfaction is measured on its own without the affective component to obtain a subjective evaluation of the quality of one's life. However, as Lyubomirsky and Lepper (1999) point out, there is a difference between evaluating your life as enjoyable, and considering yourself to be a happy person. An individual's circumstances may be very far from ideal yet the person can still continue to enjoy life. The Subjective Happiness Scale was designed to measure how happy individuals consider themselves compared to others, and to what extent their happiness is affected by what is happening in their life (SHS; Lyubomirsky \& Lepper, 1999).

\section{Eudaimonic Happiness}

In order to have a measure that captured the eudemonic type of happiness, Ryff and Keyes (1995) developed a multidimensional measurement tool that defined components of psychological wellbeing (PWB). They defined psychological well-being (PWB) as: the presence of psychological resources that enable optimal performance in the face of life challenges. The scale was designed to define PWB both theoretically and operationally; together they are called the Ryff Scales of Psychological Well-being (RSPW). The RSPW are made up of six constructs, all shown to contribute to and predict mental, emotional, spiritual and physical health. The six constructs are: autonomy, personal growth, self-acceptance, life purpose, mastery, and the ability to have positive relations with others. The following is a brief outline of the constructs: 1) autonomy: to be self-determined and independent, able to resist social pressures and evaluate self by personal standards; 2) personal growth: to see one's self as growing and expanding and open to new experiences; 3) self acceptance: to possess a positive attitude toward one's self and can accept both good and bad qualities; 4) purpose in life: to have goals and a sense of directedness and to hold beliefs that give life purpose; 5) environmental mastery: a sense of competence in managing the environment and to make effective use of surrounding opportunities; and 6) positive relations with others: to have satisfying and trusting relationships with others and to be concerned about the welfare of others. These constructs have all been shown in the literature to significantly contribute to mental health and are negatively associated with mental illness. For example, individuals with a high degree of environmental mastery feel they can make changes in their world and 
take advantage of environmental opportunities, which is related to self-efficacy, confidence, and eudemonia (Ryff \& Singer, 2006). In contrast, individuals with a low score on environmental mastery tend to attribute their lack of control to internal causes and feel helpless to prevent future negative outcomes, and as a consequence often suffer from depression (Abbott et al., 2006). All the subscales in psychological well-being have their own unique relationship with mental health.

Subjective happiness is more fleeting, episodic and short term than psychological well-being (Raibley, 2012). Nevertheless, as mentioned in the sections above, studies have shown that increasing positive emotions can also contribute to long-term gains in wellbeing. A study by Fredrickson and Losada (2005) found that individuals who experience a higher ratio of positive affect than negative affect are more likely to be in a state of 'flourishing' - a construct that includes psychological well-being. Therefore, in essence hedonic happiness can help to promote eudaimonic happiness. However, research suggests that depending on what individuals do to sustain their happiness can influence the likelihood that hedonic happiness will lead to long-term gains.

\section{It is Possible to Increase Sustainable Happiness and Wellbeing?}

According to research, we can become happier, though Lyubomirsky posits that people find it difficult to sustain happiness because people tend to look to the wrong things to maintain or sustain positive affect (Lyubomirsky, 2006). There are three major contributors in determining happiness: circumstances, genetics, and behaviour. According to research by Lyubomirsky, Sheldon and Schkade (2005), circumstances only has a 10 percent impact on how happy we are, genetics has a larger role at 50 percent, and the remaining 40 percent is based on our intentional activity. Therefore, contrary to popular belief, intentional activity has more sway than circumstances in determining happiness (i.e. Lyubomirsky, Sheldon, \& Schkade, 2005).

If a significant portion of an individual's happiness is determined by their own actions, what actions help to increase happiness? In order to answer this question, researchers have studied the behaviour of individuals high in happiness and have found common behaviours across cultures and age groups (Csikszentmihalyi, 1999; Diener, Suh, Lucas, \& Smith, 1999; Gillham et al., 2011; Janoff-Bulman \& Berger, 2000; Lyubomirsky, 2001). Some of these common behaviours are: nurturing and enjoying relationships (Martin, Reece, Lauder, \& McClelland, 2011), expressing gratitude (Wood, Maltby, Gillett, Linley, \& Joseph, 2008), engaging in helping others (Seligman, Ernst, Gillham, Reivich, \& Linkins, 2009), 
savouring life's pleasures (Bryant \& Veroff, 2007), committing to personal goals (Green, Oades, \& Grant, 2006), and seeing difficult circumstances as challenges to overcome (Maginness, 2007). Studies have found that these behaviours are predictive of happiness, and studies are starting to emerge showing that encouraging these behaviours can help to increase sustained happiness in people. This type of research is still at its infancy, however, tested interventions are starting to build an evidence base to show that these behaviours can be developed in individuals and increase their happiness.

\section{Happiness and Wellbeing Interventions}

A number of different wellbeing and happiness interventions have been developed using strategies that have arisen out of positive psychology research. Some of these strategies not only impact future well-being, but can prevent the development of depression. These interventions aim to develop participants' ability to elicit, maintain, and increase specific positive emotions in order to deliberately moderate the effects of negative emotions and build resources for future negative experiences. The interventions are concerned with avoiding negative emotions, per se, but learning to regulate them effectively with positive emotions. There are many strategies that are effective at eliciting positive emotion in times of stress. Some of these strategies can also be used when things are going well in order to build resources and to form automatic, trait-like responses to situations. The results of research on the effects of positive emotions is that some of the most successful interventions have been those that have focused on increasing savouring, gratitude, acts of kindness, flow, goal setting and individual strengths (Layous et al., 2011; Norrish \& Vella-Brodrick, 2009; Seligman et al., 2009; Sin \& Lyubomirsky, 2009). These interventions have been shown to have longterm effects on well-being and in some cases help to decrease depressive symptoms in depressed and non-depressed samples. These concepts and interventions are discussed in depth below.

\section{Savouring}

Savouring is the capacity to attend to, appreciate, and enhance pleasurable experiences (Bryant \& Veroff, 2007). Bryant and Veroff describe savouring as the positive counterpart to coping and as a form of emotion regulation that is used to prolong and enhance positive experiences (Bryant, 1989; 2003). They propose that different savouring processes regulate different positive emotional states (Bryant \& Veroff, 2007). For example, the savouring process of thanksgiving regulates gratitude in that focusing on what one is thankful for will elicit and maintain a grateful, positive state. Research has found that the ability or 
tendency to savour positive experiences can enhance the intensity and frequency of individuals' current happiness and may be a significant determinant of future wellbeing (Berger, 2000; Bryant, 1989; Bryant \& Veroff, 2007; Janoff-Bulman \& Berger, 2000; Langston,1994; Lyubomirsky \& Tucker, 1998).

The experiences in life that an individual can savour are as numerous as the varying experiences that bring each of us pleasure, and these can differ significantly between individuals, groups, and cultures (Bryant \& Veroff, 2007). We can savour sensory experiences such as: the feeling of sunshine warming the skin, the smell of freshly cut grass, the taste of a good wine, or the relaxing sound of the ocean. Yet savouring is not limited to our senses, we may savour the awareness of complex thought, such as attending to the elegance of a mathematical equation or we may luxuriate in the pleasure of our emotional response to an event, such as a family reunion. The capacity to savour involves being mindful of what is happening in the present, and it can also involve reminiscing positive experiences in the past or anticipating positive events in the future. One study found that people were who frequently savoured past positive events, and did not dwell on negative past events, were significantly happier than people who were more likely to think about past negative events or often made a contrast between the present and happier times in the past (Bryant \& Veroff, 2007).

Research on the ways of savouring has revealed a set of cognitive and behavioural responses that people use across all types of savouring domains (Bryant \& Veroff, 2007). Bryant and Veroff have identified distinct strategies as natural responses to pleasurable experiences: sensory-perceptual sharpening, sharing with others, memory building, selfcongratulation, comparing, absorption, behavioural expression, temporal awareness and counting blessings. In related research, Quoidbach, Berry, Hansenne, and Mikolajczak (2010) have found that individuals who use a wider range of savouring strategies reported higher levels of happiness in comparison to individuals who used a narrow range. Some savouring strategies and their influence on happiness are discussed below.

The savouring strategy sensory-perceptual sharpening, is used to concentrate on a particular sensory stimulus while blocking out other senses (Bryant \& Veroff, 2007). This focus helps to increase awareness of the pleasure experienced from a specific perceptual sense. For example, one might close one's eyes in order to increase the awareness and pleasure from surrounding sounds. Counting blessings is another savouring strategy that has been shown to be a significant predictor of well-being and happiness (Chadwick, 2012; Jose, Lim, \& Bryant, 2012). Counting blessings is being mindful of how lucky or fortunate one is, 
similar to having a disposition of gratitude and thanksgiving. The person pinpoints what they are grateful for, identifies the source of the blessing, and then links one's gratitude to this source (Bryant \& Veroff, 2007). The savouring technique sharing with others involves deliberately looking for people to share an experience with, and communicating to them how much it is valued or enjoyed. However, for this technique to be effective, the listener's response needs to be perceived as enthusiastic or at least interested (Reis et al., 2010). If the listener is engaged, sharing with others can build personal and interpersonal resources including promoting trust, intimacy, feelings of validation, and increased enjoyment of the listener's company. Sharing positive experiences with others has also been linked with satisfaction in life (Gable et al., 2004; Reis, et al., 2010). It has been suggested that sharing may contribute to an individual seeing themselves as positive in the eyes of others and this may lead to greater self-esteem and facilitate a more positive evaluation of one's life (Gable et al., 2004; Reis, et al., 2010). Research has shown that adolescents rely quite heavily on their social networks to help them savour and enjoy positive experiences (Meehan, Durlak \& Bryant, 1993). Interestingly, Meehan et al. (1993) found that adolescents rely on their peers more to savour and enjoy life's experiences than to cope with negative experiences.

In this vein, Jose, Lim, and Bryant (2012) examined the inter-relationships between momentary positive events, momentary happy mood, and momentary savouring (including the savouring strategies of counting blessings, perceptual sharpening and sharing with others). They found that momentary savouring mediated the relationship from positive events to happy mood on a daily basis, supporting the notion that savouring positive events increases happy mood. They also found that savouring moderated the pleasant events to happiness relationship, namely, when individuals who savoured at a high level experienced fewer positive events they still maintained a positive mood compared to individuals who savoured less. This result suggests that, even at times when positive events are few and far between, savouring is still an effective strategy to maintain positive mood.

Savouring responses are often automatic, though savouring strategies can also be developed and used intentionally. Encouraging clients in therapy to use savouring intentionally has been shown to be a useful strategy to help individuals suffering from depression to start to notice and experience pleasure in their day-to-day lives (Seligman, Rashid, \& Parks, 2006). Deficits in the ability to savour positive affect have been associated with a tendency toward depression (Carver \& Johnson, 2009). Encouraging clients to savour everyday experiences that are normally rushed through, such as breakfast or the walk to work, has been shown to decrease depressive symptoms (Seligman, Rashid, \& Parks, 2006). 
Also interventions inviting participants to savour the moment have been found to significantly decrease depressive symptoms in a non-clinical sample (Hurley \& Kwon, 2012).

\section{Savouring in Adolescence}

Savouring research has predominantly focused on adults, however a few studies have examined the strategies and impacts of savouring responses in adolescence (Bijttebier, Raes, Vasey, \& Feldman, 2012; Cafasso, Bryant, \& Jose, 1994; Chadwick, 2012). Cafasso, Bryant, and Jose (1994) investigated savouring responses in a large sample of 10 to 13 year olds in America and found that a higher capacity to savour was related to wellbeing. They also found that there were similar detectable patterns of savouring in youth as in adults, however, adolescents did not differentiate among specific types of savouring as much as adults. The lack of differentiation in adolescence could be due to the late formation of stable response styles to affective states, as these begin consolidation and become trait-like in early to late adolescence (Abela \& Hankin 2008; Mezulis, Hyde, \& Abramson, 2006). Chadwick (2012) found similar results between adults and adolescents, i.e. she found that unlike adults, adolescents struggled more to distinguish between dampening and amplifying savouring strategies. Also adolescents were not as clear with their preferences for which strategies they endorsed. These findings suggest that differentiated savouring starts to develop in adolescence, as youth become more aware of what enhances and dampens positive mood.

A recent study by Bijttebier, Raes, Vasey, amd Feldman (2012) investigated responses to positive affect in a sample of 10-14 year olds, including 'positive rumination' and dampening. (Dampening, for these authors, is "engaging in cognitive responses that are likely to counter positive affect". Positive rumination, for these authors, are "responses that are likely to maintain or intensify positive affect"). They found that low levels of positive rumination and high levels of dampening predicted depressive symptoms up to three months later. This study suggests that some savouring responses can work as a protective factor against developing depression in adolescence. Another recent study investigated if adolescent's responses to positive events would be a significant determinant to their mood and behaviour (Gentzler, Morey, Palmer, \& Yi, 2012). Grentzler and colleagues found that maximising responses to positive events by sharing, reflecting, and celebrating predicted more positive feelings four months later. On the other hand, minimising responses to positive events, by downplaying the event's significance or likelihood to recur, predicted higher levels of internalising and externalising problems over and above the effects of coping with negative events. This result is in line with literature that shows that maladaptive attributions 
to positive events are predictive of depressive symptoms (e.g., Gladstone \& Kaslow, 1995). This research provides additional evidence indicating that savouring in adolescence can increase positive affect.

Findings from Chadwick (2012) show that different savouring strategies in adolescence have different positive outcomes. High arousal savouring strategies (i.e. sharing with others and behavioural expression) were used the most by adolescents and was shown to positively predict happiness. However, self-focus savouring strategies (i.e. counting blessings and congratulating oneself) was the only strategy that predicted positive affect over time and was positively associated with the greatest number of wellbeing indicators. Additionally, Chadwick found that adolescent savouring moderated the relationship from hedonia to eudemonia, indicating that the more an adolescent savours (i.e. counts their blessings, congratulates themselves, considers how they will remember the event), the more they will feel good about themselves and the event. One could argue that hedonic happiness is transmuted by savouring into future psychological and social wellbeing.

Overall, research suggests that savouring is an effective strategy to regulate emotions and to increase happiness, but it also seems to prevent the development of depressive symptoms. Savouring starts to emerge in adolescence, when they start to learn which strategies are effective for eliciting and maintaining positive affect. The studies that are emerging suggest that savouring could be used in interventions to enhance wellbeing and buffer depression. Importantly, savouring may be one route to building psychological resilience.

\section{Gratitude}

Gratitude has featured in philosophy, religion and theological writings over many centuries as an essential civic virtue that is needed for society to function healthily, to live life well, and as an attitude that demonstrates excellence of character. It is only relatively recently that the study of gratitude has been given such close attention in psychology. The increased focus on gratitude occurred when psychologists started to consider gratitude not only as an important moral affect that promotes prosocial behaviour but also as an essential element that contributes to an individual's well-being (Snyder \& Lopez, 2005).

Gratitude can be conceptualised as a momentary affect or a long-term disposition. As an emotion, gratitude has been defined as an 'attribution-dependent state' that results from, firstly, being aware of having obtained a positive outcome and secondly, recognising that there is an external source for the positive outcome (Weiner, 1985; Emmons \& McCullough, 
2003). The positive outcome might be material or non-material and the object of gratitude may be directed to a person or to an impersonal (nature) or non-human source (god/s, the cosmos) (Solomon, 1977; Teigen, 1997). In certain kinds of gratefulness there is an element of appreciation that comes from knowing that the benefit received is undeserved or unearned, being defined as 'the willingness to recognize the unearned increments of value in one's experience' (p. 389, Bertocci \& Millard, 1963). What is not often mentioned in the literature is that experiencing gratefulness may also derive from internal experiences and may not always be directed towards a particular benefactor. For example some participants in gratitude studies have reported being grateful for their 'own abilities' or 'waking up in the morning'; these examples suggest that gratitude involves more than just interpersonal appreciation (Graham \& Barker, 1990 ; Veisson, 1999; Wood, Froh, \& Geraghty, 2010).

Gratitude as a disposition takes a more comprehensive view of gratitude and is often defined as an orientation towards noticing and appreciating the positive outcomes or experiences in life (Wood, Joseph, \& Maltby, 2008). Having an orientation of gratitude differs from hope or optimism as these focus on possible positive outcomes that the future may hold whereas gratitude focuses on what one has now or has had in the past. Research on dispositional gratitude has found that gratitude is related to, but distinct from, trait measures of positive affect, vitality, and optimism (McCullough, Emmons, \& Tsang, 2002). Although gratitude overlaps with other positive feelings, it also manifests a unique pattern of appraisals that distinguishes it from happiness (Weiner, 1985). Measures have been developed based on the wide conceptualisation of gratitude (i.e. as a trait) as an orientation to life, some of these scales include: the Gratitude Questionnaire (GQ: McCullough et al., 2002), the multifactorial Gratitude, Appreciation, and Resentment Test (GRAT: Watkins, Woodward, Stone, \& Kolts, 2003), and the multifactorial Appreciation Scale (Adler \& Fagley, 2005). The Gratitude Questionnaire is one of the most frequently used scales for measuring gratitude, and it measures individual differences in frequency, density, and intensity of gratitude (McCullough et al., 2002).

People who score highly on measures of gratitude tend to experience more positive outcomes than those who demonstrate less gratitude. Gratitude has been linked to sleep quality and duration (Wood, Joseph, Lloyd, \& Atkins, 2009), and lower levels of physical illness (Emmons \& McCullough, 2003) and depression (Wood et al., 2008). Also there is also a wide body of literature showing that gratitude helps to promote and maintain positive relationships (Algoe, Haidt, \& Gable, 2008). As gratitude is associated with many of the characteristics needed for the development of relationships such as: willingness to forgive 
(DeShea, 2003), low narcissism (Farwell \& Wohlwend-Lloyd, 1998) conflict resolution and increase in reciprocity of helpful behaviour (Baron, 1984; Tsang, 2006). It seems that a grateful response to life and all the challenges that it can bring, is an adaptive psychological response that can bring about many positive effects. It has been posited that gratitude may counteract the natural occurring 'adaptation to satisfaction' by fostering an awareness of how fortunate one is, regardless of one's circumstances (Frijda, 1988). Having the capacity to daily notice and appreciate different aspects of life is viewed as an essential component to happiness and causal factor of wellbeing (Bryant, 1989; Janoff-Bulman \& Berger, 2000; Langston, 1994). Wood, Maltby, Gillet, Linley and Joseph (2008) conducted meditational analysis investigating the relationship between gratitude and wellbeing. In their study wellbeing was defined by obtaining a low score in depression and stress, and a high score on perceived social support. Wood et al. (2008) assessed undergraduate university students on a range of different variables at the start and end of their first term. The authors found that after testing different models, that gratitude scores at the beginning of the term lead to wellbeing at the end of the term. In other words students that were high in gratitude were more likely to have positive outcomes at the end of the term.

People who score highly on measures of gratitude tend to experience high levels of life satisfaction and wellbeing (Froh, Kashdan, Yurkewicz, Fan, Allen, \& Glowacki, 2010) and they often display frequent prosocial behaviour (Algoe, Haidt, \& Gable, 2008). A wide body of literature shows that gratitude helps to promote and maintain positive relationships (Algoe, et al., 2008). Gratitude is associated with many of the characteristics needed for the development of healthy interpersonal relationships, for example, willingness to forgive (DeShea, 2003), low narcissism (Farwell \& Wohlwend-Lloyd, 1998), conflict resolution, and increase in reciprocity of helpful behaviour (Aron, 1984; Tsang, 2006). It seems that a grateful response to life and all the challenges that it can bring is an adaptive psychological response that can bring about many positive effects. It has been posited that gratitude may counteract the naturally occurring 'adaptation to satisfaction' by fostering an awareness of how fortunate one is, regardless of one's circumstances (Frijda, 1988). Having the capacity to notice and appreciate different aspects of life on a daily basis is viewed as an essential component to happiness and causal factor of wellbeing (Bryant, 1989; Janoff-Bulman \& Berger, 2000; Langston, 1994).

Given all the positive aspects of gratitude, therapists and researchers have conducted interventions to determine if increasing the expression of gratitude can bring about positive change in an individual. One type of intervention is the use of gratitude diaries. Gratitude 
diaries usually involve participants writing down three things daily that they are grateful for, over a set number of days or weeks (Emmons \& McCullough, 2003). One study found that gratitude diaries reduced worry to the same degree as thought monitoring and cognitive restructuring (Geraghty, Wood, \& Hyland, 2010). Geraghty and colleagues (2010) also found a higher retention rate with participants using gratitude diaries than participants using standard cognitive techniques. The authors suggest that gratitude diaries may be more effective in reducing anxiety than techniques used in usual practice. Another intervention that has shown to be successful is writing a letter of gratitude. A recent study found that participants who wrote three letters of gratitude over three weeks had significant increases in happiness and life satisfaction, and decreased depressive symptoms, compared to the control condition (Toepfer, Cichy, \& Peters, 2011).

\section{Gratitude in Adolescence}

Children can start to display gratitude at a young age but it is normally around the age of 10 years that gratitude matures (Park \& Peterson, 2006a). At around 10 years of age children's empathy develops enough for them to comprehend others' intentions, and that these intentions can affect their own happiness (Bono \& Froh, 2009; Park \& Peterson, 2006a). Therefore it is only by early adolescence that individuals can fully experience gratitude and reap all of the possible benefits. The development of gratitude in early adolescence can help to secure and build important resources, especially in regards to social interactions. Learning to express gratitude can help one to gain assistance and cooperation from others and can help to establish supportive and fulfilling relationships. Having access to these relationships can then lead to numerous positive opportunities and useful knowledge.

Accordingly it has been posited that one of the ways gratitude increases well-being is through social integration (Froh, Bono, \& Emmons, 2010). In Froh's et al.'s (2010) study, social integration was defined as being passionate about helping and feeling connected to others at a macro level (e.g. community) (p.4). Social integration has been positively associated with a higher grade point average, life satisfaction, positive affect, self-esteem, hope, and happiness; and negatively associated with depression, envy, delinquency, and antisocial behaviour (Froh et al., 2010). Froh et al (2010) found that gratitude at one point in time predicted social integration six months later. The authors used mediational analysis to examine the mechanisms that were involved in the path from gratitude to wellbeing. They found that prosocial behaviour mediated the relationship from gratitude to social integration. This result indicates that gratitude stimulates prosocial behaviour, which in turn helps 
adolescents connect to their wider community.

Interventions designed to increase gratitude in order to promote wellbeing in adolescents are starting to emerge. Froh, Sefick, and Emmons (2008) ran a gratitude intervention for two weeks with 11-13 year olds, at middle school, as part of their mandatory curriculum. Students were randomly assigned to a gratitude condition, hassles condition, or a control group (where students had class as normal). Students in the gratitude condition were asked to list up to five things for which they were grateful, and students in the hassles condition were asked to list up to five things that annoyed or bothered them. The authors found that the gratitude condition helped to increase subjective wellbeing and decrease negative affect, in comparison to the hassles condition, but not in comparison to the control condition. However, the gratitude group did experience greater satisfaction with school experience at three weeks follow up, compared to both the control and hassles condition. Also, further analysis indicated that the gratitude condition enhanced students' grateful responses towards those from which they received help, compared to the hassles condition.

Froh has expressed concern that many of the gratitude interventions that show success, both in adult and adolescent populations, only do so when contrasted with a negative control condition or to techniques used in therapy (Froh, Kashdan, Ozimkowski, \& Miller, 2009). He hypothesised that a possible reason behind this was because gratitude interventions are more effective on individuals low in positive affect. Froh and colleagues tested this hypothesis and found evidence to support it. Eighty-nine children and adolescents were randomly assigned to a gratitude intervention or a no-treatment control condition. Students were given 10-15 minutes daily for 5 days to either write a gratitude letter or in a journal about daily events. Findings indicated that for youth low on positive affect, the gratitude condition increased gratitude and positive affect at post-treatment and increased positive affect at 2-month follow-up, significantly more than the control group. However, as hypothesised, youth high in positive affect showed no positive gains from the gratitude intervention. These findings suggest that gratitude interventions may only be helpful to adolescents who are unhappy. However it is too soon to come to the conclusion that gratitude interventions can only influence those low in positive affect, especially given the many positive associations found with gratitude. What is needed is further investigation into the various strategies of gratitude with adolescents, while measuring a variety of positive outcomes, with the inclusion of no-treatment controls.

Research indicates that having a grateful perspective in life is an adaptive psychological response that can lead to many positive outcomes, including health benefits, a 
buffer to depression, and an increase in wellbeing and happiness. Gratitude can be a vital resource for adolescents as it can help to create and foster important relationships and broaden interpersonal options. Gratitude interventions are still in their infancy and may need to improve on their quality of procedure in order to capture the full range of effects possible.

\section{Kindness}

Kindness is the quality of being friendly, generous, and considerate (Oxford Dictionaries, 2012). The demonstration of kindness can be shown in countless ways but it is the warm and caring nature of the act that makes it kind (Baldwin \& Baldwin, 1970). What is initially clear when reviewing the literature on kindness is that the research is not as robust compared to many other constructs in psychology. Much of the debate and investigation in the literature is targeted at related constructs such as altruism, generosity, and empathy. Kindness has been predominately studied as a virtue that contributes to the morality of society and promotes prosocial behaviour. Accordingly, kindness has been used as a measure to assess the development of moral judgement and reasoning in children (Kohlberg, 1976; Lamborn, Fischer, \& Pipp, 1994; Piaget, 1965). Young children define kindness in very concrete, situation-specific ways. As children mature they start to identify people's intentions behind their actions. By adolescence most individuals are able to integrate behaviours, underlying intentions, varying perspectives, and conflicting motives and needs into a sensible interpretation of human interactions (Baldwin \& Baldwin, 1970). More recently in some areas of research including education and positive psychology, kindness has been used as an intervention to increase prosocial behaviour and psychological well-being (Lyubomirsky, Sheldon, \& Schkade, 2005; Otake, Shimai, Tanaka-Matsumi, Otsui, \& Fredrickson, 2006; Seligman et al., 2009; Smith, 2001; Souza \& McLean, 2012).

Kindness interventions in the classroom are a relevant topic of discussion that is taking place in some venues of education because of the prosocial implications, benefits to learning, and overall well-being (Seligman et al., 2009; Smith, 2001; Souza \& Mclean, 2012). The environment that many children and adolescents experience at school is often one that promotes unkindness to gain or keep status among peers. Often among students bullying is accepted implicitly as normative behaviour and a natural response to conflict or the unknown. As a result the classroom does not always feel like a safe place for students, and this can be detrimental to learning and building positive relationships, not to mention student's happiness (Boulton \& Smith, 1992; Sylwester, 1995). The optimal environment for students to learn in is one that promotes positive emotions; research shows that positive affect broadens attention, 
drives exploration, and leads to creative problem solving (Ashby, Isen, \& Turken, 1999; Seligman et al., 2009) .

In Australia a project called 'The Act of Kindness' was implemented in 2005 in primary schools, with the aim to decrease the prevalent bullying in South Australian schools (The Act of Kindness, 2012). Marion Parsonage created this project in response to the discrimination and bullying that was evident, especially against minority groups. 'The Act of Kindness' now runs in more than 40 schools throughout Australia. The aim of the project is to promote positive connections and relationships between children, and in particular develop acceptance of those from minority groups. The project encourages students to commit kind acts in their school, and each week teachers identify the kindest acts and the student is awarded with a certificate at the end of the week. Teachers, parents and principals have reported that the project has begun to change their communities for the better and many parents have continued to sponsor the programme once they noticed the change in their own children (The Act of Kindness, 2012).

Souza and McLean (2012) used two schools as case studies to investigate the positive impacts of the Act of Kindness programme. They found that staff attitudes began to change in regards to the benefits of recognising kindness in their students. The programme helped raise awareness of kindness as a positive behaviour and in turn increased kindness towards others. It helped students reflect on their own behaviour, and their attention also shifted to the minority populations in their school. The authors point out that the recipient of the kind act is likely to show their gratefulness in some way to the giver. The demonstration of appreciation on its own can be a reward to the giver, creating an intrinsic motivation for students to be kind and creating a positive relational cycle. Souza and McLean (2012) infer that once this cycle is set in motion, kindness can become a natural disposition, so that students behave kindly because of the satisfaction they receive from being kind rather than any external motivation. Therefore kindness can help to develop prosocial behaviour in students by raising awareness of the impact of their behaviour on others and the rewards gained in being kind to others.

Research has continually found that there is a strong link between the ability to connect with others and well-being (Baumeister \& Leary, 1995; Martin, Reece, Lauder, \& McClelland, 2011). Since kindness helps to develop positive interpersonal relationships, it is not surprising that kindness has been found to buffer against symptoms of depression (Gillham et al., 2011). Gillham et al.'s (2011) longitudinal research revealed that kindness, along with 'other-focused' strengths, negatively predicted depression in adolescents for up to 
two years. That is, students who were rated high in kindness at the beginning of high school were less likely to have depressive symptoms two years later compared to those that scored lower on kindness at the beginning of high school. Additionally their mediational analysis suggested that kindness helped to increase social support, which in turn buffered individuals against depression. This result is in line with other research that has found that high altruism in individuals negatively predicts thoughts of death or self harm (Fanous, Prescott, \& Kendler, 2004), and it is also congruent with research that has found that social support can act as a protection against developing depression. The research shows that if an individual has a tendency to care for others, they are less likely to suffer from low mood or harm themselves.

Research has investigated whether increasing an individual's kindness can increase their happiness. Lybomirsky and colleagues (2005) conducted two randomised controlled kindness interventions with American university students. In the first intervention participants were asked to perform five acts of kindness one day a week or disperse their kind acts out throughout the week for six weeks (Lyubomirsky et al., 2005). The results showed that happiness levels increased for those participants who performed all of their acts of kindness in one day each week. but did not increase for those who spread them out during the week. The authors concluded that for those participants who spread their acts of kindness out during the week, their kindness did not stand out from their normative behaviour, therefore it did not produce a significant impact. On the other hand, for those participants who performed all of their acts of kindness on one day, this profile differed enough from their normal behaviour for the intervention to have an effect.

In the second intervention some students were invited to perform the same acts over a period of 10 weeks, and others were asked to choose different activities every time (Boehm, Lyubomirsky, \& Sheldon, 2008). The participants who could not vary their acts of kindness became less happy midway through the intervention and went back to their baseline happiness levels post-intervention. In contrast, the participants who were able to vary their acts of kindness showed significant increased levels of happiness midway through the intervention and up to one month after the intervention. Both kindness interventions supported the notion that kindness can enhance a person's happiness and also suggest that timing and variety is crucial to the impact that acts of kindness will have.

Otake and colleagues (Otake et al., 2006) investigated the relationship between kindness and subjective happiness with university students in Japan. Otake et al. (2006) proposed that kindness has three components: the motivation to be kind, recognising kindness 
in others, and acting kindly towards others. The results of their first study showed that happy people scored higher than the unhappy group in the strength of kindness (i.e. motivation, recognition and acts of kindness). Interestingly the content of these happy experiences for the happy group were often centred on the kindness received from other people.

Otake et al. (2006) state that becoming more aware of kindness in yourself and others fosters kindness as it increases an individual's wish to be kind to others and to start to recognise themselves as a kind person. They predicted that this increase in kindness would lead to an increase in happiness due to the nature of kindness discussed earlier. Otake et al. (2006) proceeded to conduct a second study where they invited participants to write down their own acts of kindness each day for one week. Otake et al. (2006) found that participants' happiness levels significantly increased compared to the control group for up to one month after the intervention. They also found that those participants whom had the greatest change in happiness reported more grateful experiences and engaged in more acts of kindness than those than had a smaller change in subjective happiness after the intervention. These studies by Otake et al. (2006) reveal that happiness can be increased by observing one's own kindness and this pathway may be due to an increase in motivation to be kind, recognition of kindness in others and one's self, and an increase in acts of kindness.

Kindness promotes prosocial behaviour, can help to build relationships, and increase positive emotions; all of these lead to happiness through multiple pathways and help to prevent depressive symptoms from developing later in life. Kindness interventions for children and adolescents can be pivotal to academic and social development, impacting on youth's current and future happiness. Also it is possible that kindness increased through interventions may develop into an inherent, stable characteristic. Interventions aimed at increasing acts of kindness can help to increase the benefactor's happiness; however it is important that the individual completes a number of activities on a single day so that the action stands out from their normative behaviour. Also it is important that the individual varies the types of activities they do for others so that it does not become a routine task. One of the mechanisms by which kindness leads to happiness is by becoming more aware of kindness in others and oneself.

Flow

Flow is a state of complete absorption and optimal functioning in an activity that is intrinsically enjoyable (Csikszentmihalyi, 1999). There is no need for an external reward in this state because the person is operating at full capacity and the experience itself is reward 
enough. Flow requires the challenge of a task to be matched with the skills necessary to meet the challenge; flow occurs when a person finds the "sweet zone" between her competence and the challenge at hand(Moneta \& Csikszentmihalyi, 1996). Hence, an artist may find herself in flow when painting, or an athlete while competing. However, flow can also be experienced in everyday tasks. Flow activities studied and reported in the literature range from composing music to engaging in housework (Asakawa 2004; Csikszentmihalyi, 1988; 1990; Han 1988). When in flow, anything that is not related to the task is forgotten. The individual experiencing flow does not experience stress, worry, or self-consciousness. Absolute focus is achieved, there is an increase in confidence and a sense of mastery, and the perception of time is altered. Similar concepts related to flow such as 'engagement', 'peak experiences' and 'optimal functioning' have been discussed in the literature for some time. However, flow theory originates from Csikszentmihalyi's $(1982 ; 1990)$ extensive research. The concept emerged from qualitative interviews with individuals describing their optimal experiences. Some of the people interviewed included sculptors, extreme sport enthusiasts, and basketball players. The interviewees characterised their optimal experiences as described above, and Csikszentmihalyi labelled this state 'flow' because of its effortless yet focused control. Flow is also seen to be part of the concept autotelic, meaning an experience where the activity is valuable in itself.

Flow has been categorised as an optimal state that increases positive affect and helps to build resources that contribute to psychological well-being (Csikszentmihalyi, 1999; Nakamura \& Csikszentmihalyi, 2002a; 2002b; 2008). The state of flow has been consistently associated with positive affect in cross-sectional (e.g. Eisenberger, Jones, Stinglhamber, Shanock, \& Randall, 2005) and longitudinal research (e.g. Shernoff, Csikszentmihalyi, Schneider, \& Shernoff, 2003). Researchers have found that repeated exposure to flow experiences increases positive mood (Csikszentmihalyi, 1999; Eisenberger et al., 2005), however how flow has this effect is not entirely clear. According to Csikszentmihalyi (1999), all resources are so focused on the task at hand during flow that there is no cognitive space to reflect on subjective states. In fact, being happy might be a distraction from flow. However, after engaging in flow, people report that they experienced exhilarating levels of happiness (Csikszentmihalyi, 1999). Additionally people who experience flow frequently report that they have more purpose and meaning in their lives than individuals who experience less flow (Adlai-Gail, 1994; Hektner, 1996). Researchers have also found that flow is positively correlated with self-esteem (Adlai-Gail, 1994; Wells, 1988) and a sense of fulfilment and life satisfaction (Asakawa, 2004; Bryce \& Haworth, 2002; Clarke \& Haworth, 1994; Han, 1988; 
Ishimura \& Kodama, 2006; Peterson, Park \& Seligman, 2005). Furthermore flow has been shown to predict resilience (Schmidt, 2003) and psychological well-being (Cantor \& Sanderson, 1999; Kubovy, 1999; Massimini \& Carli, 1988; Rathunde, 1988; Steele \& Fullagar, 2009). In one study, Japanese college students who experienced flow frequently in their daily lives were more likely to report higher self-esteem, lower anxiety, and use active coping strategies compared to students who experienced flow less frequently. They were also more likely to report active commitments to college life, greater satisfaction with their lives, and reported more jujitsu-kan, a Japanese sense of fulfilment (Asakawa, 2009).

Additionally, interest and engagement have been strongly associated with measures of psychological health, especially experiences of chronic interest (Hunter \& Csikszentmihalyi, 2003) as opposed to chronic boredom which has correlates to psychological dysfunction (Hunter \& Csikszentmihalyi, 2003). Hunter and Csikszentmihalyi (2003) compared the experiences of youth who were interested and engaged in everyday life with those who were bored and restless. They found that interested participants had greater self-esteem, optimism, and internal locus of control, and lower pessimism than bored participants. This finding suggests that flow and engagement have positive consequences for adolescent wellbeing.

However, flow does not seem to ensure long-term happiness or psychological wellbeing, the beneficial effect of flow seems to be dependent on the types of activities an individual is engaged in. If an individual limits herself to a narrow range of flow activities that promotes isolation from others, inhibits growth, becomes addictive or are destructive, then these flow experiences will not contribute to her psychological well-being but instead may result in negative consequences (Csikszentmihalyi, 1993; Csikszentmihalyi \& Rathunde, 1998; Inghilleri, 1999). Activities that can stimulate growth, bring meaning, and offer opportunities to learn and increase skill are predictive of psychological well-being (Csikszentmihalyi, 1999). For example, flow experiences in academic work are associated with commitment and achievement (Carli, Fave, \& Massimini, 1988; Csikszentmihalyi et al., 1993; Heine, 1996; Nakamura, 1988), and have been shown to maximize efficiency and creativity (Csikszentmihalyi, 1988; 1990; 1997; Nakamura \& Csikszentmihalyi, 2002a; Steele \& Fullagar, 2009) and predict psychological well-being (Steele \& Fullagar, 2009). Therefore flow is predictive of future psychological well-being in the context of meaningful activities and goals.

Accordingly, interventions aimed at increasing flow experiences that incorporate meaningful activities should also increase psychological well-being. Csikszentmihalyi (1997) proposes two types of flow interventions: those that assist individuals in finding flow and 
those that shape the activity or environment to be conducive of flow. Assisting individuals to find flow can be achieved by helping individuals identify their strengths and interests and using activities related to these characteristics. Also, flow can be created in monotonous activities (e.g. washing dishes) by making them more meaningful and stimulating. If the individual imposes a challenge on a task that will require skill and is enjoyable, then arguably this challenge can create a flow experience. An example of this dynamic is given in Lyubomirsky 's (2007) book, a man would try and tap in time to a very fast bass line in the music he was listening to. He found that when he tried to do this activity, time raced by while he was waiting in traffic and frustration and negative thoughts would fade away. This type of flow has been labelled microflow; it may not have as much an impact as macroflow, but it still seems to be effective.

The second type of intervention, shaping activities to be conducive of flow, involves ensuring the pre-conditions for flow are in place during the activity in which individuals will be participating. Csikszentmihalyi's $(1997 ; 1999)$ research identified three core preconditions for flow: 1) establishing a clear goal - certainty and clarity on what one intends to $d o ; 2)$ unambiguous feedback- knowing how well one is performing during the performance itself; and 3) balance between skill and challenge -a sense that one is engaged in challenge equal with one's current ability. These pre-conditions are suggested by Csikszentmihalyi to be used as guidelines for interventions.

Flow is an optimal state for learning as it enhances concentration, interest, and enjoyment. Concentration is central to flow, it allows the instigation of flow, and increases the flow experience (Csikszentmihalyi, 1990). Concentration is related to meaningful learning (Montessori, 1967), and fosters greater depth of cognitive processing and academic performance (Corno \& Mandinach, 1983). Interest, however, steers attention, it signals intrinsic motivation, and helps to maintain engagement. Research has found that interest is significantly related with school achievement (Hidi, 1990; Schiefele, Krapp, \& Winteler, 1992). Finally, enjoyment is related to creative achievement and school performance (Csikszentmihalyi et al., 1993; Nakamura, 1988). Shernoff and Csikszentmihalyi (2006) investigated student engagement in schools using their own research and a meta-analysis of the literature from private and public schools in various countries including Finland, Denmark, Japan, and America. They found that if challenges and skills were perceived to be high by the students, then the students would be twice as likely to be focused and engaged in the activity. Also, individuals experiencing a match between skill and challenge felt higher motivation, positive mood, and self-esteem. 
As mentioned before, one of the fundamental factors of flow is the balance between challenge and skill. If the challenge is too easy, the individual will become bored and if the challenge is too difficult, the individual will either get anxious or frustrated.

(Csikszentmihalyi, 1990; Csikszentmihalyi \& Rathunde, 1993). Furthermore, when individuals participate in a given activity over time, they generally become more proficient. This development of proficiency will require new challenges and skills for the individual so that the activity continues to be flow-inducing. This growth-enhancing aspect of flow has many positive repercussions for the individual, including the enhancement of self-efficacy, confidence, and mastery. Therapy has directly utilised this growth-enhancing aspect of flow to help individuals recover from depressive symptoms, to promote social integration, and to cultivate meaningful life challenges (Delle Fave \& Massimini, 1992; Inghilleri, 1999; Massimini, Csikszentmihalyi, \& Carli, 1987; Massimini \& Delle Fave, 2000). Therapeutic interventions informed by flow theory have been shown to be successful across diverse cultural settings (Inghilleri, 1999).

Seligman expresses the view that increasing meaningful flow experiences is core to experiencing an engaged life, which he defines as a significant path to wellbeing (Seligman, 2002). Lyubomirsky (2007) affirms this contention, explaining that flow helps us to enjoy the present moment and keeps us involved and challenged by life. Furthermore it helps to create a positive and strong sense of self by developing a sense of control and mastery over our lives. Though interventions to increase flow still need to be developed, research suggests that it is possible to increase flow in our daily lives in order to promote an engaged life.

\section{Goal setting}

Goal setting is a two-part process: it is deciding what one wants to do and then deciding how to achieve it. This process is a necessary part of daily life, from deciding on what to have for breakfast to deciding on a vocation. Consequently the types of goals an individual chooses and how he or she goes about achieving them will affect every aspect of his or her life. Researchers have recognised the importance of goal setting in regards to adaptive life choices, positive functioning, and well-being (Green, Oades, \& Grant, 2006; Sheldon, Kasser, Smith, \& Share, 2002). In Masten et al.'s (2004) longitudinal study, future motivation and planning was found to be one of the strongest predictors for resilience evidenced in individuals up to 20 years later in their life. Studies have found the positive benefits of goal setting do not only lie in goal achievement but also in the processes of achieving a goal (Watson, Hubbard, \& Wiese, 2000). Having goals and attempting to 
accomplish them give a sense of meaning and control in our lives (Cantor, 1990; Sheldon et al., 2002). However, sometimes when an individual reaches a goal they have set, they can feel suddenly aimless or disillusioned. Either the goal did not bring them the satisfaction they hoped for or they now have nothing else to live for. The effect of disillusionment after accomplishing a goal reveals the importance of the type of goal that an individual chooses and the expectation behind the goal (Lyubomirsky, 2007).

Kasser and Ryan (1996) identified two types of life goals: intrinsic and extrinsic goals. Intrinsic goals are those developed out of inherent psychological needs for autonomy, competence, and relatedness (Kasser, 2002; Niemiec, Ryan \& Deci, 2009). Intrinsic goals are chosen by the individual because of their personal meaning and the process of completing the goal is reward in itself. Some examples of intrinsic goals include wanting to participate in volunteer work, aiming to master an instrument for personal enjoyment, or working on self development. Extrinsic goals are imposed by others, rely on the reaction or evaluation of others, and are usually a means to an end. Extrinsic goals are set by external sources in society such as the media or culture. For example, aiming to be rich, famous, and attractive are extrinsic goals. The media often portray these extrinsic goals as a sure route to happiness, though research has shown that a major focus on extrinsic goals detracts from and probably reduces well-being (Sheldon \& Elliot, 1998). Alternatively pursuing and achieving intrinsic goals is associated with higher life satisfaction and happiness, higher self-esteem and open mindedness, and fewer experiences of depression, anxiety, and general health problems (Kasser \& Ryan, 1993; 1996; Sheldon \& Kasser, 1995). Kesser and Ryan (1996) explain that the reason intrinsic goals lead to well-being is because the individual is meeting their psychological need for either connecting with others, developing autonomy, or competence. In support of this view, Sheldon and Elliot (1998) found that individuals who set and pursued intrinsic goals had higher levels of self-regulation and happiness than individuals who pursued goals because of external pressures or rewards.

Importantly, however, cross-cultural studies show slightly different outcomes and associations with extrinsic and intrinsic goals. Ingrid, Majda, and Dubravka (2009) point out that in collective cultures individuals often subordinate their intrinsic goals for extrinsic goals for the good of the group, whereas in individualistic cultures an individual is encouraged and free to pursue intrinsic goals. The motivation behind extrinsic goals, especially financial success, for example, seems not to be the same between these two types of cultures. Financial success is commonly associated with consumption or security in individualistic cultures, whereas in collective societies financial success is often associated with opportunity, self- 
growth, and the ability to help others (Ingrid et al., 2009). Additionally many collective cultures live in poor or transitional countries and pursuing extrinsic goals is sometimes a matter of survival, meeting basic needs and providing the possibility to pursue intrinsic goals. This difference in socioeconomic environment and culture could explain why extrinsic goals have been shown to be predictive of well-being in nations such as China (Lekes, Gingras, Philippe, Koestner, \& Fang, 2009), Russia (Ryan et al., 1999), Hungary, and Macedonia (Martos, \& Kopp, 2012; Spasovski, 2009). In addition to noting cultural implications, these studies highlight that the motivation behind a goal and meaning attached to it may be more influential to an individual's well-being than the content of a goal.

Goal pursuit and attainment in adolescence is pivotal to developing identity, direction and establishing present and future well-being. Lightfoot, Stein, Tevendale, and Preston (2011) found that goal setting, decision making and self-reliant coping were robust protective factors for homeless youth. Youth that evidenced an ability to set goals were less likely to engage in problem behaviours such as risky sexual behaviour, antisocial behaviour or substance abuse. In a sample of Zambian adolescents, personal goals in the areas of power, affiliation and achievement were significantly associated with greater life satisfaction (Hofer \& Chasiotis, 2003). Lekes et al. (2010) found that extrinsic goals did not predict poor wellbeing in their sample of Chinese and American adolescents, rather, their research found that endorsement of extrinsic goals predicted risky behaviours such as substance use. The authors suggest that extrinsic goals may not predict poor well-being initially with adolescents, but may negatively impact them in the long-term. Adolescents who value wealth and physical pleasures higher than other goals have been found to be at greater risk of developing various kinds of psychological disorders such as disruptive and personality disorders (Cohen \& Cohen, 2001). It is important to note that the above studies only demonstrate associations, the findings are based on concurrent, not longitudinal measurements, therefore the direction of these relationships cannot be stated with certainty. It is likely that there is a two-way relationship between goals and their outcomes.

Since goals can contribute significantly to adolescent's wellbeing or contribute to further maladaptive and risky behaviour, it is important to understand the factors that influence the goals adolescents choose and the process involved in attaining them. Family, peers, educational environment, social economic status and culture all set the stage for the development, attainment, and experience of goals. Parents' aspirations for their children, support of their child's autonomy, and their involvement and acceptance will influence the content of goals set by adolescents and the child's experience of goal attainment (Behnke, 
Piercy \& Diversi, 2004; Marjoribanks, 1993; 2003). Adolescents can be extremely influenced by their peers when it comes to goal setting; the type and degree of peer support, peer prosocial behaviour and peer expectations are associated with prosocial goal seeking and goal attainment at school (Wentzel, 1994; Wentzel, Filisetti, \& Looney, 2007; Barry \& Wentzel, 2006). Students from low socio-economic backgrounds often aspire to improve their life status, but relative to their more advantaged peers, their aspirations are not as high. Also, those from low socioeconomic backgrounds often have lower beliefs in their ability to achieve their goals (Carroll, 2002; Gordon Rouse, 2001). Ethnicity and culture has been shown to shape goal content in adolescence. In collective cultures, value is often placed on the effort that is put into obtaining a goal rather than the ability to obtain the goal, whereas in individualistic cultures people are not so much concerned with the effort but rather the ability to obtain a goal. It is more concerning in an individualistic culture if a goal is not achieved due to lack of ability rather than lack of effort (Phinney, Baumann, \& Blanton, 2001). These differences may have an impact on how adolescents will experience pursuing goals and what they consider failure or success. Therefore goal content, pursuit and attainment is shaped by many different factors in adolescence. Though many of these factors cannot be changed by the individual, it does suggest that goal pursuit and attainment are learnt and therefore are open for intervention.

Early adolescence appears to be the opportune time to intervene in helping individuals become more aware of their goals and learn how to pursue helpful goals. A meta-analysis revealed that goal setting usually increases during early adolescence (Nurmi \& Pulliainen, 1991) and confidence in attaining goals increases from mid-adolescence (Nurmi, 1994). The content of goals in adolescence is also age-related, in that young adolescents typically pursue leisure goals and that older adolescents start to focus on academic, family and property goals. Youths can become more aware of their goals and the impact they have on their life. Their understanding can be developed in regards to: what are beneficial goals to pursue, what will contribute to their immediate and long-term wellbeing, and how to go about pursuing these goals.

In light of the positive results that goal setting can achieve, it is of no surprise that goal setting is often included in therapeutic interventions and group prevention programmes as an essential element to positive change. Setting goals can help the client or participant to focus and specify what they want to achieve and then to make steps towards achieving them. As a result, the client can see their own progress enhancing their self-confidence and selfefficacy. An example of a depression preventative programme that incorporates goal setting 
as one of its key strategies is the LARS\&LISA programme developed in Germany (Wahl, Patak, Pössel, \& Hautzinger, 2011). The aim of the programme was to improve adolescent's life skills, foster their realistic thinking and to influence school behaviour. The programme consisted of five main elements, one of them being the formulation and setting of personal goals. When compared to another prevention programme and teaching as usual, the LARS\&LISA programme helped to decrease symptoms of depression and aggressive behaviour and increase social networking in both German and American schools. However, this difference in outcome cannot be fully attributed to the goal setting strategy, as it was one of the five main elements to the programme.

Interventions that use goal setting as the sole strategy for change are less common, especially with adolescents. Sheldon, Kasser, Smith and Share are some of the few researchers that have specifically used goal setting as an intervention. Sheldon et al. (2002) randomly assigned university students to either a goal-training program or to a control condition. Although there were no main effects of program participation on later goal attainment, they found that overall goal progress helped to increase participants' vitality, psychosocial well-being, affect-balance, and personality integration. The authors concluded that goal striving can provide a proactive route to well-being and personal growth. Another intervention by Sheldon and colleagues aimed to increase university-based community members' subjective happiness using a 6-month goal setting experiment (Sheldon et al., 2009). Participants were randomly assigned to set goals either to improve their life circumstances (i.e. comparison condition) or to increase their feelings of autonomy, competence, or relatedness in life (i.e. treatment conditions). The results showed that participants who set goals that contributed to relatedness, competence or autonomy showed increases in happiness (Sheldon et al., 2009). However, only those who continued to be engaged in their goals gained positive benefits. Those who did not complete their goals obtained lower scores in happiness compared the control condition.

Simply setting and pursuing goals is not sufficient for obtaining happiness and wellbeing. Research has found that there are certain variables that help goals become more attainable and effective in increasing well-being. Individuals who pursue goals based on their intrinsic value are happier than individuals who chase extrinsic goals set by popular culture. There are many factors that contribute to the formation, pursuit and attainment of goals, suggesting that it is complex but also showing ways that goal setting can be learnt and used in interventions. Goals can lead to enhanced well-being or risky behaviours and in some cases detract from well-being, depending on the content and method of attainment. Intervening in 
adolescence may be pivotal as the goals young people pursue will help shape who they will become and determine their life path.

\section{Character Strengths}

A character strength has been defined as "a pre-existing capacity for a particular way of behaving, thinking, or feeling that is authentic and energising to the user, and enables optimal functioning, development and performance" (p.9, Linley, 2008). According to Peterson and Seligman (2004), a character strength is different to a character trait in that there is a moral tone connected with a strength; a strength is valued in its own right, and does not diminish others in order to function and contribute to individual fulfilment.

Peterson and Seligman (2004) set out to define and categorise strengths that enable human flourishing in the same way that the Diagnostic Statistical Manual has categorised psychological disorders that disable human functioning. The authors drew on major world philosophies, religions and a host of empirical studies to look for ubiquitous human virtues and strengths. Peterson and Seligman averred that an exhaustive set of moral characteristics considered necessary for individual happiness and meaning could be identified. The authors labelled these set of strengths Virtues In Action (VIA), which are explained and expanded on in the manual entitled Character Strengths and Virtues: A Handbook and Classification (CSV; Peterson \& Seligman, 2004). The manual contains a detailed classificatory system of six core moral virtues: wisdom/knowledge, courage, humanity, justice, temperance, and transcendence; and 24 subordinate empirically measurable character strengths (Peterson \& Seligman, 2004). For strengths to be included in the VIA, they had to meet a list of criteria, such as: contributing to individual fulfilment, not diminishing others' integrity, valued in its own right, and not considered as a means to an end. The strength needed to be widely recognized across cultures and the deliberate target of societal practices and rituals that try to cultivate it. These strengths are said to be evolutionary, already observable from the age of three (Peterson, 2006a), trait-like and manifest in a range of behaviours, thoughts and feelings. As such they are measurable and generalisable across situations. Research findings to date have supported the use of the VIA strengths classification as a robust and valid representation of character strengths across cultures and differing age populations (BiswasDiener, 2006; Linley et al., 2007; Park, Peterson, \& Seligman, 2004; Shimai, Otake, Park, Peterson, \& Seligman, 2006; Steger, Hicks, Kashdan, Krueger, \& Bouchard, 2007).

Seligman and Peterson have developed a VIA questionnaire for individuals to identify, use and develop their strengths. Numerous studies have found that identification and 
use of character strengths is associated with and predictive of higher well-being, and many other adaptive outcomes including higher levels of energy, vitality and self-esteem. In a study with 214 university students as participants, Govindji and Linley (2007) found that the use of strengths predicted unique variance in subjective well-being and psychological well-being. Proctor, Maltby, and Linley (2009) reported similar findings in their study; people who used their strengths more reported higher levels of subjective well-being, confidence, self esteem and psychological vitality.

There is still little known about the relationship between character strengths and wellbeing however one study looking at this relationship found that goal achievement was a strong mediator between VIA strengths and well-being (Linley, Nielsen, Wood, Gillett, \& Biswas-Diener, 2010). Structural equation modelling analysis revealed that using strengths is associated with higher goal progress, which is associated with greater need satisfaction and in turn well-being. In other words, people who used their strengths to achieve their goals were more likely to attain their goals and were happier than those who did not use their strengths. As mentioned in the goal section, achievement of intrinsic goals helps to meet psychological needs, which increases well-being. The Linley et al. (2010) study shows that one of the ways strengths help to increase well-being is through helping individuals achieve their intrinsic goals.

Park and Peterson (2006) developed the VIA-Youth in order to include items in the questionnaire that were developmentally appropriate for adolescents. The VIA-Youth has four subscales: temperance strengths (e.g., prudence, self-regulation), intellectual strengths (e.g., love of learning, curiosity), transcendence strengths (e.g., hope, spirituality, love), and other-directed (interpersonal) strengths (e.g., kindness, modesty). Research suggests that these different strengths have different positive outcomes, for example, transcendence strengths such as spirituality, gratitude, and hope have been related to well-being and life satisfaction in adolescents (Froh et al., 2010; Sawatzky et al., 2009; Snyder et al., 2003). On the other hand, intellectual strengths (e.g., love of learning, creativity, curiosity) have been shown to help school engagement, and interpersonal strengths have been associated with social integration and the establishment of a positive social support network (Shoshani \& Slone, 2012).

Shoshani and Slone (2012) conducted a longitudinal study with four middle schools in Israel to assess which strengths helped students transition adaptively from 7 th to 8th grade. They found that despite the decrease in subjective well-being due to middle-school adjustment, students' intellectual strengths were found to be significant predictors of positive 
emotions and cognitive adjustment to school. They also found that interpersonal strengths were found to be a significant predictor of social adjustment. In Gillham et al.'s (2011) study of students transitioning from 9th to 10th grade in American schools, transcendence strengths predicted higher happiness and interpersonal strengths predicted low symptoms of depression. Additionally low levels of interpersonal strengths predicted the onset of high levels of depression and suicide ideation during the follow-up period, while adolescents with high interpersonal strengths did not report elevated depression or suicidal ideation during the follow-up period. Research has also found that character strengths can protect adolescents from developing mental disorders, having interpersonal difficulties and becoming engaged in risky or anti-social behaviours, including substance abuse, and violence (Bromley, Johnson, \& Cohen, 2006).

Therefore interventions aimed at developing adolescents' character strengths have the potential to not only increase well-being but also help to prevent the development of maladaptive behaviour and psychological disorder. Adolescence is an opportune time to intervene, as this is when morals, personality and character strengths become more pronounced, shaped and set (Hart \& Carlo, 2005; Park \& Peterson, 2006a). At around age 12 moral judgements become more developed as individuals begin to interpret their social environment in new ways (Piaget, 1965). Other-oriented judgements and perspective taking becomes more enhanced in adolescence, which impacts on their interpersonal skills and development of altruistic tendencies (Eisenberg, 1986; Eisenberg, Zhou \& Koller, 2001; Kohlberg, 1981; Underwood \& Moore, 1982). According to Park and Peterson (2009), even if interventions target just one character strength it can affect or even enable other strengths.

Identifying and developing character strengths is a core component of the classroom interventions developed by Seligman and colleagues at Geelong Grammar School in Australia (Seligman, et al., 2009). The classroom intervention is part of a Positive Psychology programme that is incorporated into the school curriculum with the aim to increase students' well-being and school engagement. Students take the VIA Signature Strengths test to help them identify their strengths and the curriculum offers strategies to assist students in applying their strengths in new ways and to overcome challenges. Preliminary results show that the positive psychology programme increased students' reports of enjoyment and engagement in school. Additionally, according to teacher reports, the programme improved students' strengths related to learning and engagement in school (e.g., curiosity, love of learning, creativity). The programme also impacted on students' interpersonal strengths, according to mothers' and teachers' reports, and the programme also 
improved the social skills of the students (e.g., empathy, cooperation, assertiveness, selfcontrol). The evaluation of the programme is still being undertaken and there is no systematic data reported as of yet, however it appears that the outcomes look promising.

The activities that Seligman uses in the programme at Geelong Grammar School have been adapted from a web-based intervention with adults. Seligman, Steen, Park, and Peterson (2005) designed a character strength intervention where they asked participants to use their strengths in a new and different way every day for one week. Participants reported higher levels of happiness and lower levels of depression for up to six months after the intervention. Seligman et al.'s (2005) study has been replicated by Gander, Proyer, Ruch, and Wyss (2012). They found similar results, up to six months after the intervention participants reported higher levels of happiness and lower depressive symptoms than the placebo control group.

The VIA has shown to be an effective measure of character strengths. It seems that certain strengths are determinative of various facets of wellbeing. One of the ways in which character strengths has been found to lead to wellbeing is through helping individuals ascertain intrinsic goals. Research by Seligman suggests that helping adolescents to identify and use their strengths can have a significant impact on their enjoyment and engagement in school, as well as interpersonal interactions. Related research suggests that developing character strengths could also have a significant impact on wellbeing and depressive symptoms.

\section{Positive Approaches to Life (PAL)}

All the elements discussed above, savouring, gratitude, kindness, flow, goal setting and character strengths, were incorporated into the PAL programme. The interventions and strategies that have been shown to be most successful in each area were created into separate sessions to create PAL. The aim was to develop positive resources in PAL participants in order to increase their ability to face challenging circumstances. The following sections discuss the lack of programmes similar to PAL, other gaps in the literature, and how this current study aims to help fill this gap.

\section{Gaps in Current Positive Psychology and Prevention Programmes}

A growing number of positive psychology programmes are aimed at treating/preventing depression or increasing wellbeing in general, however, many of these are internet-based or are aimed at adults. Some schools have started to incorporate positive psychology strategies into their school curricula (Seligman, et al., 2009), though at this time 
there are very few school-based preventative programmes that target at-risk youth. Also educational programmes may increase the general wellbeing of students, however, it is important to assess if they will help individuals at risk. As of yet, there seems to be no school-based intervention for at-risk students that incorporates all of the different types of useful positive psychology strategies into a single programme. If the aim is to build resilience, then it is important to give the individual a variety of positive strategies as resources they can use, rather than focus solely on a single strategy. Any given strategy will not be effective for all individuals, whereas teaching a variety of methods produces a greater chance that the programme will be more generally effective.

Additionally, the majority of prevention/resilience or wellbeing programmes do not measure personal resilience. Therefore it is difficult to establish if personal resilience was increased due to intervention and if this had any impact on the decrease in depression or increase in wellbeing. An intervention that seeks to build resilience against depression endeavours to facilitate permanent changes within the individual, to develop resilient processes so that these processes become internal and more stable. Therefore, if an individual is developing resilient processes, then it would be helpful to know if personal resilience has been enhanced and how this may impact on other outcomes.

Another issue is that many of the programmes tested have not always followed rigorous evaluation methodologies. For example, many have not used a control group that does not participate in any type of programme, or there is no long-term follow-up to determine the programme's impact over time (Froh et al., 2008; Linley et al., 2006; Ruini, Belaise, Brombin, Caffo, \& Fava, 2006). Furthermore, some prevention programmes have combined positive psychology techniques with CBT strategies, and though they have been shown to be effective, it is difficult to know which element(s) of the programme has/have brought about change (e.g. Rashid \& Anjum, 2008).

A recent literature search has only identified one wellbeing programme that has used a combination of intentional positive psychology activities, and it only used those techniques to build a capacity for positive emotions (see Savage, 2011). Additionally, this programme was not directly aimed at the goal of preventing depression and consequently did not measure depressive symptoms. Rather its sole aim was to increase subjective wellbeing (SWB) in 1012 year olds who had previously yielded low scores in SWB. The results of the study indicated that the 10 week SWB programme increased positive affect and life satisfaction significantly at four months post intervention. The SWB intervention was associated with increased life satisfaction. However, Savage (2011) found that expectancy, child-rated 
alliance, social self-efficacy, but not the SWB intervention per se, significantly led to positive affect.

Thus, a significant gap in the literature still exists: research is needed to establish the efficacy of a wellbeing programme that uses a variety of intentional positive psychology activities in a long-term design with a control group, and also assesses both negative and positive outcomes, including personal resilience.

\section{The Present Study}

The present study aimed to compare two school-based prevention programmes with a control group. Both intervention programmes ran for 12 weeks; one programme was the Kiwi ACE programme, which uses a CBT orientation, and the other was the newly developed Positive Approaches to Life (PAL), which uses a combination of positive psychology strategies that have been shown in the literature to increase wellbeing and mitigate the effects of depression. In order to select students for the three groups, a screening questionnaire that measured depression, strengths and difficulties, resilience, and other positive psychological constructs was completed by a large sample of Year 9 students (13-year-olds). Students were selected as "at-risk" if they yielded a mild to moderate depression score. The at-risk students were invited to take part in the programme at school in groups of ten, or were placed in the waiting-list control group. A school counsellor from the school and a clinical psychologist jointly administered the intervention sessions. The same questionnaire that was used for screening was then used for follow-up immediately at the close of the 12-week programme, after six months, and then one year after the programme ended.

The purpose of this study was to determine, firstly, if both programmes would be effective at promoting positive outcomes, including resilience and preventing depression. The second purpose was to examine if there would be any differences between the outcomes of these two programmes, and if so, what these differences would be. Both programmes were examined for possible mediational relationships, namely investigating which mechanisms intervened between the interventions and the outcomes. The chief aim of this study was to determine which techniques would lead to certain outcomes, e.g. resilience and wellbeing, and which factors help to promote these outcomes.

\section{Predictions}

The following predictions were made for this current study: 


\section{Kiwi ACE Intervention}

\section{Prediction 1}

Based on prior research from Woods $(2006 ; 2011)$, it was predicted that Kiwi ACE would decrease participants' depressive symptoms up one year after the programme ended.

\section{Prediction 2}

Based on prior research from Kowalenko et al. (2005), it was predicted that Kiwi ACE would decrease participants' maladaptive coping up to one year after the programme ended.

\section{Prediction 3}

Based on prior research from Kowalenko et al. (2005), it was predicted that Kiwi ACE would increase participants' adaptive coping up to one year after the programme ended.

\section{Prediction 4}

Based prior research on the maintaining factors of depression and on the aims of CBT strategies (Andrews \& Wilkinson, 2002; Gillham, 2003; Spirito, et al., 2011), it was predicted that Kiwi ACE would help to decrease participants' reports of total difficulties (peer problems, conduct problems, hyperactivity, and emotional difficulties) up to one year after the programme ended.

\section{Prediction 5}

Based on prior research regarding the protective and risk factors associated with resilience (Hjemdal, et al., 2006) and on interventions aimed at increasing resilience (Gillham et al., 1990), it was predicted that Kiwi ACE would increase participants' resilience up to one year after the programme ended.

\section{Mediational relationships}

\section{Prediction 6}

Based on prior research on the maintaining factors of depression and on the aims of CBT strategies (Andrews \& Wilkinson, 2002; Gillham, 2003; Spirito et al., 2011), it was predicted that a decrease in difficulties (peer problems, conduct problems, hyperactivity, and emotional difficulties) would mediate the relationship between Kiwi ACE and depression. 


\section{Prediction 7}

Based on prior research on the maintaining factors of depression and on the aims of CBT strategies (Andrews \& Wilkinson, 2002; Gillham, 2003; Spirito et al., 2011), it was predicted that a decrease in maladaptive coping (e.g. avoidance, denial) would mediate the relationship between Kiwi ACE and depression.

\section{PAL Intervention}

\section{Prediction 8}

Based on Fredrickson's $(1998 ; 2001)$ broaden-and-build theory and prior research related to the determinants of psychological well-being (PWB) (Fredrickson \& Losada, 2005; Ryff \& Singer, 2006), it was predicted that PAL would increase participants' wellbeing up to one year after the programme ended.

\section{Prediction 9}

Based on findings from similar interventions which have shown an increase in satisfaction with life post-intervention (e.g. Savage, 2011), it was predicted that PAL would increase participants' satisfaction in life up to one year after the programme ended.

\section{Prediction 10}

Based on Fredrickson's $(1998 ; 2001)$ broaden-and-build theory and prior research showing the determinants of subjective happiness (e.g. Sheldon \& Lyubomirsky), it was predicted that PAL would increase participants' happiness up to one year after the programme ended.

\section{Prediction 11}

Based on research that indicates intentional gratitude activities can increase participants' gratitude (e.g. Froh et al., 2008), it was predicted that PAL would increase participants' gratitude up to one year after the programme ended.

\section{Prediction 12}

Based on prior research that indicates that building a capacity for positive emotions helps to build personal resilience (Ong et al., 2006; Tugade \& Fredrickson, 2004), it was predicted that PAL would increase participants' resilience up to one year after the programme ended. 


\section{Prediction 13}

Based on interventions that have found positive intentional activities leading to decreases in depressive symptoms (e.g. Layous et al., 2011), it was predicted that PAL would decrease participants' depressive symptoms up to one year after the programme ended.

\section{Mediational relationships}

\section{Prediction 14}

Based on research indicating that positive emotions help to increase future gains in wellbeing (Fredrickson \& Losada, 2005, Tugade, et al., 2004), it was predicted that subjective happiness would mediate the relationship between PAL and the increase in wellbeing.

\section{Prediction 15}

Based on research that indicates that gratitude helps to build psychological resources (Froh, 2010), it was predicted that gratitude would mediate the relationship between PAL and the increase in wellbeing.

\section{Summary of predictions}

Therefore it was predicted that PAL and Kiwi ACE would both decrease depressive symptoms and increase resilience but that only PAL would increase the remainder of the positive outcomes. It was predicted that Kiwi ACE would help to decrease depression through decreasing maladaptive coping strategies and difficulties. In contrast, it was predicted that PAL would help to increase psychological well-being through building a capacity for positive emotions. 


\section{Chapter 2: Method}

\section{Programme Design}

Two interventions were conducted in this study: Kiwi ACE (Adolescents Coping with Emotions) and the Positive Approaches to Life programme (PAL). Kiwi ACE is a programme developed in Australia and it has been trialled in Australia and in New Zealand with success. The current operationalisation is a replication of a small-scale study performed by Dr. Barbara Woods in 2005 on the Kiwi ACE programme. In addition to this replication, the PAL was created and included in the design in order to compare and contrast these two theoretical approaches. The Kiwi ACE programme focuses on ways to improve efforts to cope with problems, whereas PAL was designed to build capacity for happiness and wellbeing.

This was a Quasi-Experimental Design, it was not possible to enact a pure experimental design because both programmes and a control group could not be run in every school. The majority of the schools involved only had enough resources to run one programme group in their school, additionally in most schools there were only enough 'atrisk' students to form one programme group and one control group. Additionally, in two schools there were only enough 'at-risk' students to form one programme group, these schools did not have enough students to have a control group.

\section{Programme Structure}

Both programmes included the same sessions on Problem Solving, Assertiveness, and Negotiation and Compromise. These shared sessions were targeted at building resilience, and were considered to be compatible with both a CBT and positive psychology approach. Kiwi ACE included six sessions based on the main CBT strategies and once these were taught, they were incorporated throughout the subsequent sessions and reviewed each week. The PAL included six sessions based on six different positive psychology techniques in place of the CBT techniques.

\section{Structure of Sessions}

The main structure of the sessions has been taken from the Kiwi ACE programme. Both programmes consisted of twelve one-hour sessions. The structures of each session in both programmes were similar across programmes and across sessions. The following layout was consistent for every session: 
The aim: The aim is presented in a text box at the very beginning of each workbook chapter and is usually one or two sentences. This short description is to let the leaders know the aim of that particular session to help them begin the session with a clear focus and to help maintain focus. In turn this helps to ensure that the main points are put across to the students.

Review: Each session begins with a review of the previous session and a discussion on the set tasks practiced during the week. This approach is to help students consolidate lessons they have learnt and to learn from experiences others have had during the week. It is also a time for the leaders to gauge if students have practiced any of the activities during the week. The review is usually five minutes long.

Games: The majority of the sessions start with a game, and these are usually ten minutes long. Games are an important part of the session, they can help to gain students' focus and interest and provide opportunities for students to develop their personal and social skills while having some fun (Cheung, 2006). Also many of the games introduce the topic of the session in an interactive and non-threatening way.

Activities: The main content of each session was broken down into a mix of written, oral and movement-based activities to keep students interested and accommodate all learning styles (i.e. Dunn et al., 1995). The majority of activities were 10 minutes and never longer than 20 minutes to keep students engaged, this is particularly important as short attention spans are common in at-risk students (American Psychiatric Association, 2000). The first activity of each session aimed to gather information from the students about what they already know on the session's topic. The student's contribution is then incorporated into the remainder of the session, helping students become part of the lesson and to own the information and lessons learnt.

To practice: Each session ended with a section on the set activities to practice during the week. This rehearsal was designed to consolidate what the students have learnt and to help generalise the skills learnt to everyday life.

\section{Programme Development and Content}

\section{Development of Kiwi ACE}

Kiwi ACE is based on an Australian-designed programme that was named "Adolescents Coping with Emotions" (or ACE). ACE was developed by Dr. Ann Wignall, Janne Gibson, Nicole Bateman and Ron Rapee (1998), with support from Macquarie 
University, Child and Adolescent Psychiatry, Northern Beaches Adolescent Service and the New South Wales Department of Education and Training. ACE was selected as a government initiative for high risk students by the Australian national Mind Matters programme (Australian Institute for Primary Care, 2005). The ACE programme deployed in Australia was found to reduce depressive symptoms and increase coping skills for 82 female participants aged between 13-16 years (Kowalenko et al., 2005). The study involved an unequal distribution of males across the intervention and waitlisted groups, therefore no results were available for males. When Kiwi ACE was trialled in New Zealand in 2005, it was found to decrease depression in male and female 13-year-olds, including Māori and Pacific Island students (Woods, 2008).

\section{From ACE to Kiwi ACE}

Dr Barbara Woods adapted ACE with the aim of increasing its effectiveness and making it culturally appropriate in the New Zealand context. Woods consulted with five Māori and Pacific mental health professionals, with two kaumatua (elders) representing the Māori community and with two student groups that had representation of Māori, Pacific, Asian and New Zealand European ethnicities. The process of consultation raised some important issues and suggestions, and as a result the following changes were made: Māori and Pacific names such as "Aroha" and "Seli" were used for two of the characters appearing in the student workbook, cultural designs specific to New Zealand replaced those in the Australian books, the name "Get Real" which was given to the cognitive restructuring worksheets were replaced with the term "Realistic Thinking" (students felt that the term "Get Real" was coming from an adult being a "try-hard").

Woods made further changes to increase the relevancy to New Zealand young people for whom family connections have a strong influence. For example, in the original 'Realistic Thinking worksheet', students were asked to think of a more "balanced way to think of an issue" this was replaced with "what would your friends and family say?" Additional changes were made in response to the Australian ACE trial (Kowalenko, et al., 2005), which showed that use of non-productive coping strategies increased. In response to this finding, Dr Woods decided to include a section on "Changing Bad Habits" aiming to put forward the concept that habits are 'learnt behaviour' and that bad habits can be 'unlearnt', also that relapse is normal and to ensure that students knew where to go for help. Woods designed this section drawing from Sobell's Guided Self-Change Programme (1993), and techniques from Motivational Interviewing (Miller \& Rollnick, 2002). 


\section{Current Changes to Kiwi ACE}

Session times in Kiwi ACE were changed from 80 minutes to 60 minutes in order to fit with class periods, consequently there were 12 sessions instead of eight. The format used for Kiwi ACE in this current study was similar to the original format in ACE.

Feedback from the school counsellors that used Kiwi ACE in 2005 found some of the Cognitive-Behavioural Therapy (CBT) content in Kiwi ACE to be too wordy and complex for the students. In response to their feedback, the 'realistic thinking' form ${ }^{1}$ was simplified to make the format less cluttered and the content easier to follow. Also the structure of sessions two, three and four were changed; only one or two new concepts of CBT were introduced at one time. The instructions and explanations in the handbook and workbook were rewritten to make them as straightforward as possible.

The pictures in the Group Book were replaced with more modern illustrations to keep the programme relevant to students. The word 'unhelpful habits' was used rather than 'bad habits' as 'unhelpful' is usually perceived as easier to change than a behaviour that is labelled "bad". The words: "to practice" instead of "homework" or "home tasks" were used to avoid the negative connotations that 'homework' has for many adolescents.

\section{Theoretical Underpinnings to Kiwi ACE}

Kiwi ACE is a preventative depression programme that aims to build adolescent's coping skills and decrease depressive symptoms by using personal and social competence training with a cognitive behaviour approach. The underlying theory behind the cognitive behaviour approach is that thoughts cause feelings, therefore changing unhelpful thought patterns can change negative moods, feelings and behaviour (Bieling, McCabe, \& Antony, 2006). This approach aims to help students to be aware of the link between their thoughts and feelings and to provide tools to replace negative and unhelpful thought patterns with helpful thoughts in order to cope more effectively and improve their mood. This approach addresses the cognitive attributional style students use to appraise both present and future events and the causality of these events. Recognising and challenging faulty thinking patterns has the potential to impact on many dimensions related to depression - on belief in the self, somatic symptoms and social problems and enable the teenagers to better deal with individual stress and life events (Spirito et al., 2011).

\footnotetext{
${ }^{1}$ See Appendix A: Realistic Thinking Form, from Kiwi ACE Group Book, p.21
} 
Kiwi ACE uses a set structure in the students' workbooks called 'realistic thinking' to help explain the link between thoughts, feelings and behaviour, and targets the negative thoughts that are common in adolescents who suffer from depression. For the first four weeks, a new component was added to this structure each week, aiming to build students' knowledge on cognitive restructuring in a simple, repetitive fashion that is made relevant to their everyday lives. In the following sessions, these techniques are incorporated into the competency building which aims to develop problem solving skills, social skills, assertiveness, negotiation and compromise and also focuses on enhancing self-esteem. The programme also uses games, role-plays, rehearsals, structured discussions and written activities to teach Cognitive-Behavioural techniques, personal and social skills. Students are able to practice these skills in the safety of the group and are given opportunity to learn from and support one another.

\section{Session content of Kiwi ACE}

\section{Session One: Introduction - Stress and My Body}

The aim of the first session is to introduce Kiwi ACE, to create rapport amongst the group and to build the students' ability to identify their emotions. The session begins with students being welcomed into the group, and the main ideas, structure, and timetable of Kiwi ACE are discussed. Students and leaders play a 'get to know each other' game and group rules are established, with students deciding what the rules should be. Common problem situations are discussed, aiming to help students not feel alone in their difficulties. Students use their group books to write down what stresses them out about school, their friends and family. They identify what stress feels like to them, they have a choice of identifying physical, behavioural and emotional symptoms. Students start a log book where they record an event that happened to them, label the emotion that went with it, and use a scale to rate the emotion. The session ends with a game called 'feelings bingo' that aims to extend students' vocabulary in order to label their emotions. Students are encouraged to record events, their emotions and rate the emotion during the week.

\section{Session Two: Thinking and Feeling}

The aim of the second session is to develop students' understanding of the link between their thoughts and feelings, to understand what can cause and maintain negative feelings, and to acknowledge that we all deal with feelings differently. The session begins with a review of the last session and the leader enquires as to whether anyone practiced the 
set tasks. This is followed by a game named 'describing yourself' which aims to help students and leaders to get to know each other better. The first step of cognitive restructuring is introduced where students are encouraged to connect thoughts and feelings. The leader tells a dramatic story about an ambiguous situation that happened to them, as a group they identify the possible feelings that may have occurred as a result and identify the thoughts that may have led to those possible feelings. Students do a similar activity in their group book, where they are given an event e.g. "Sam's girlfriend Leilana recently broke up with him" and they complete the sentences "Sam could think..." "Sam could also think..." and "Sam could feel..." "Sam could also feel...". This exercise encourages students to think of a few alternative ways about an event and the feelings that these thoughts may cause. This phase is followed by a discussion on external and internal causes of feelings and a further discussion on how everyone experiences a variety of emotions and that it is normal to experience very strong emotions in different ways. The session ends with the leader encouraging students to practice writing down events that they may have difficulty with and their accompanying feelings and thoughts during the week.

\section{Session Three: Thoughts aren't Facts}

The aim of the third session is to help students link thoughts, feelings and behaviour and begin to challenge their own thoughts. The session begins with a review of the previous session and of tasks practiced during the week. The leader introduces the concept of 'realistic thinking' by using the same structure used in the last session, that is, identifying the possible thoughts and feelings associated with an event but with the added steps of identifying what is fact and what is a thought and rating how realistic the thought is. This process is performed as a structured discussion which emphasises that thoughts are not necessarily facts. Students are put into pairs and given a role play scenario which they act out and then write down the thoughts and feeling of the other character. Students then share with the rest of the class what they wrote down in their role plays. The aim of the activity is to build students' ability to see events from another person's perspective and to increase alternative ways of thinking. The session ends with a review of what students have learnt and are assigned with the task of practicing these skills during the week - specifically changing their own thoughts when they are feeling very strongly about a situation.

\section{Session Four: Detecting Evidence}

The aim of session four is to develop the students' ability to challenge their thinking. The session starts with a review of the previous session and students sharing their experiences 
of challenging their thoughts during the week. Students play the 'detective game' which introduces the main points of detective thinking. The game is followed by a review of the previous steps of realistic thinking with the addition of 'detective questions' which are: 'What happened the last time you felt like this?' 'How likely is it that this will happen again?' 'Is there another reason why this happened?' 'What would your friends/family member say if they heard you thinking this?' The leader pretends to be a teenager at a party who thinks that no one likes him/her, and the students use the detective questions to help the leader challenge his/her thinking that no one likes him/her. Then students complete the 'realistic thinking form' using a situation they have experienced when they felt down. The realistic thinking form is in their group books and includes all the steps in the previous sessions and in session four. The session ends with students being encouraged to use the 'realistic thinking form' when a challenging situation arises sometime during the week.

\section{Session Five: Problem Solving}

Session five begins with a review of session four and a discussion of the 'realistic thinking form' that students completed during the week. Students are put into two teams to play 'scavenger hunt', with the aim of building team work and introducing the topic of the session. After the game, students discuss what they would have done differently and what strategies worked the best. The leader talks about how there are some situations in life where it is difficult to come up with solutions and it can help to have some steps to follow, and then introduces the six problem solving steps proposed by the programme. ${ }^{2}$ Students use the six problem solving steps to come up with a solution on how to divide an unequal amount of chocolate bars with the group, and then discuss how the process went. After the chocolate bar game, students work through a problem (suggested by a student) as a group using the problem solving steps, and then in groups of three using the problems provided in the facilitator's handbook. ${ }^{3}$ At the end of the session there is a section on the importance of rewarding yourself and having fun. Students play the 'miming fun game' where one student mimes an activity they enjoy and the others guess what it is. Students also write down the things they enjoy doing in their groups books. The session ends with students being assigned

\footnotetext{
${ }^{2}$ See Appendix B: Problem Solving Steps from the Kiwi ACE Facilitator's Handbook, p. 43.

${ }^{3}$ See Appendix C: problems to solve from the Kiwi ACE Facilitator's Handbook, p. 44.
} 
to use the problem solving steps for problems that arise during the week and to fill out the fun activities form. ${ }^{4}$

\section{Session Six: Thinking Errors and Changing Unhelpful Habits}

The aim of session six is to identify thinking errors and deeply held beliefs, and to help students to begin changing unhelpful habits. The session begins by reviewing the previous session and tasks practiced during the week. Students then play the 'Alphabet game'. The leader introduces the concept of 'thinking errors' and goes through common thinking errors such as: 'expecting the worst', 'I should...' and 'blaming yourself'. Students use their group books to identify thinking errors that they make and decide more helpful ways of thinking. The group book activity is followed by a section on changing unhelpful habits. The leader discusses how unhelpful habits such as eating too much junk food or smoking can affect our mood and how thinking errors can make us think that these habits are helpful. Students choose one habit they would like to stop and list all the things that are good and not so good about that habit. They set a realistic goal for the next week that will help them towards changing their habit. An action plan is drawn up that includes students identifying their possible 'triggers' and deciding on a strategy to manage their risk. Placing this section half way through the programme allows for ongoing support and the opportunity for students to adjust or change their goals each week in order to assist them in changing their habit. The session ends with students being encouraged to work on the goals and action plan they set for that week.

\section{Session Seven: Social Skills}

The aim of session seven is to help students develop their social skills and identify 'thoughts' that influence social interactions. The session begins with a review of the previous session, and the individual goals and action plans that were carried out during the week. The game entitled 'people scrabble' is followed by a discussion on what it feels like to meet someone new and the thoughts and behaviours that can accompany those feelings. The goal of this discussion is to highlight the fact that temporary embarrassment and nervousness is worth the risk of making new friends. Students brainstorm what they consider to be poor social skills and good social skills, and this helps the leader to establish where the group is at and it provides the opportunity to reinforce ideas about positive and appropriate social

\footnotetext{
${ }^{4}$ See Appendix D: Fun Activities Form, from the Kiwi ACE Student Group Book, p. 34.
} 
behaviour. Students then take turns at role playing good social skills with other members in the group. This activity aims to help students identify key strategies that they can use in everyday life. Students brainstorm and share strategies that they use to meet new people. Role plays are used to tackle difficult social situations that group members have experienced using strategies that were raised earlier. Through the session the leader draws attention to the possible thoughts behind avoiding social situations or poor social interactions, such as: 's/he won't like me' or 'no one would want to be my friend'. The session ends with the leader challenging everyone in the group to attempt to meet at least one new person during the next week.

\section{Session Eight: Assertiveness}

The aim of session eight is to develop students' assertiveness. The session begins with a review of the previous session and a discussion on students' attempts at meeting new people during the week, also the leader checks in on the progress made with the goals set to change unhelpful habits. Assertiveness is introduced by differentiating it from its polar opposites: aggression and passivity. As a group, the students come up with examples of appropriate assertive behaviour, both verbal and non-verbal. Situations where it may be unsafe to be assertive are discussed and the differing cultural views on assertiveness are also discussed. Students read through assertive scripts in their group book that look at the different ways of saying 'no' when you need to. The group is then split into three teams: the passive, aggressive, and assertive teams. Each team acts out the same scenario which involves passengers telling a reckless driver to slow down, the aggressive team presents their version first, then the passive team, and finally the assertive team. The role plays are followed by a discussion on what strategies worked best. If there is still time, students practice being assertive in pairs by role-playing scenarios provided in the facilitator's handbook. Some examples of these scenarios are: 'A friend has borrowed your Playstation and he has had it for a while and you want him to bring it back tomorrow' and 'Saying NO to something your friends are doing that you don't agree with'. The session ends with students being encouraged to practice being assertive during the week and to write down two situations where they were assertive.

\section{Session Nine: Negotiation and Compromise}

The aim of session nine is to help students develop their skills in negotiation and compromise. The session begins with a review of the previous session and students are given the opportunity to share how they have been assertive during the week. Students play 
'Marooned Island' which provides many opportunities for negotiation and compromise as two teams have to decide what characters they would like to be marooned on an island with. After the game the two teams discuss their methods of negotiation and compromise. The connections between assertiveness and negotiation and compromise is discussed, followed by a group brainstorm on the important components to successful negotiation. The leader then introduces seven steps of negotiation ${ }^{5}$ tying in the group's contribution from the brainstorm. Using the seven steps of negotiation, the group works through a situation where negotiation is needed. In pairs, students role-play scenarios that require negotiation and compromise. The main ideas of the session are discussed and summarised, the session ends with students being encouraged to practice the skills they have developed and to fill out the negotiation form in their group books ${ }^{6}$ sometime during the week.

\section{Session Ten: Deep down Beliefs \& Self-esteem}

The aim of session ten is for students to use their 'realistic thinking skills' to tackle their 'deep down beliefs' and to increase their self-esteem. The session begins with a review of the previous session and the opportunity for students to share their experiences of using negotiation and compromise during the week. The leader explains the concept of 'core beliefs' and how they can affect what we think about ourselves and events that happen to us, some examples of core beliefs that are given are 'I am not worth loving' and 'I'm stupid'. The leader continues to explain underlying beliefs and ways that students can learn to identify them, leading the students into a discussion on how to challenge core beliefs with 'realistic thinking' strategies. Then the session moves to self-esteem, how self praise can be a difficult but useful skill and looking at core beliefs that can rob or boost self-esteem. Students discuss in pairs and as a group beliefs ${ }^{7}$ that rob and boost self-esteem. This exercise is followed by going through the steps that boost self esteem ${ }^{8}$ and paying particular attention to step one respect and care for yourself. Students then list in their group books what they are good at and what they like about themselves. The session ends with students being encouraged to use 'realistic thinking' to challenge one core belief and to think about situations through the week where "robbing self-esteem thoughts" occur.

\footnotetext{
${ }^{5}$ See Appendix E: Seven Steps of Negotiation, from the Kiwi ACE Facilitator's Handbook, p. 71.

${ }^{6}$ See Appendix F: Negotiation Form, from Kiwi ACE Student Group Book, p.58.

${ }^{7}$ See Appendix G: Beliefs that Rob and Boost Self-esteem, from Kiwi ACE Group Book, p.62.

${ }^{8}$ See Appendix H: Steps to Boost Self-esteem, from Kiwi ACE Group Book, p.63.
} 


\section{Session Eleven: Review of Kiwi ACE}

The aim of session eleven is to review the skills learnt in Kiwi ACE. The session begins with reviewing the previous session and discussing students' experiences of challenging their beliefs during the week. Students are then divided into four groups and each group is allocated with a Kiwi ACE Idea sheet. ${ }^{9}$ Each group is instructed to teach the rest of the group one Kiwi ACE concept in any way they choose to, and students are encouraged to be as creative as they like. After these mini-lessons, students brainstorm possible future stresses that they may experience and some coping ideas to manage them. Students are provided with a list of contacts of the supports available if they need help with anything in the future. The session ends with the students sharing what they have gained from Kiwi ACE and a farewell party is thrown for them by the leaders.

\section{Session Twelve: Survey and Review}

The session starts with a short discussion on how things are going for the students and what skills they have been practicing. Then students complete the survey they completed at the beginning of the programme once more.

\section{Development of PAL}

Positive Approaches to Life was developed using the same general structure as Kiwi ACE but substituting the CBT techniques with positive psychology techniques. As mentioned above, Kiwi ACE and PAL shared sessions that did not use Cognitive-Behavioural techniques. The shared sessions included: Problem Solving, Assertiveness, and Negotiation and Compromise. However, there were a few activities in these three sessions that used Cognitive-Behavioural techniques and these were adapted accordingly, this is explained below in more detail under the appropriate sessions. Some activities and discussions from the social skills session and self esteem session in Kiwi ACE were put into the corresponding sessions in PAL, as these were considered to be important elements of the programme and did not include Cognitive-Behavioural techniques. A few of these sections were altered to make them more compatible with PAL, and more detail about this is given below.

Sessions in PAL were made as comparable as possible to Kiwi ACE, in both format and process. The sessions that were the same or similar were put in the same order as in Kiwi

\footnotetext{
${ }^{9}$ See Appendix I: Kiwi ACE Idea Sheet, from Kiwi ACE Facilitator's Handbook, pp.89-92.
} 
ACE. The problem solving session in both programmes was placed as session five, and the following sessions were in the same order in both programmes.

\section{Theoretical Underpinnings of PAL}

Like Kiwi ACE, PAL was designed to be a depression preventative programme that aims to build adolescent's coping skills and decrease depressive symptoms. However, it does not directly target depressive symptoms but aims to increase well-being and decrease depression by using a variety of positive psychology techniques. These techniques develop habits and skills that increase happiness and well-being but they were also expected to exert an indirect dampening impact on depressive symptoms. For example, practicing gratitude is a skill that PAL aims to develop; from the literature (Froh, Sefick, \& Emmons, 2008) we know that people who practice gratitude are more likely to be happier and more resilient against developing depression compared to people who report having less gratitude in their everyday lives. Each session in PAL (apart from the sessions mentioned above) use positive psychology techniques that have been shown to be successful in increasing well-being and decreasing negative effect, and in some cases depression.

As with Kiwi ACE, PAL uses games, role-plays, rehearsals, structured discussions and written activities to teach the positive psychology techniques, social skills, and personal skills. Students are able to practice these skills in the safety of the group and are given opportunity to learn from and support one another.

The Positive Approaches to Life programme was trialled with a group of year nine volunteers at a school in the Wellington region. Adjustments were made according to the students' feedback. Additionally, time allocations and details from the activities were also adjusted to improve functionality.

\section{Content of PAL Sessions - Differences from Kiwi ACE}

\section{Session One: Introduction - Variety of Emotions}

The aim of session one is to introduce the PAL programme, to build rapport within the group and to develop students' understanding about their emotions and emotional responses. This session is very similar to session one in Kiwi ACE, however, PAL includes a description of positive emotions as well as negative emotions with the aim of building a holistic view of well-being. In Kiwi ACE, students discuss and write down situations they find stressful, and students use a stressometer, whereas in PAL students discuss situations that they enjoy in addition to writing down situations that they find stressful. Students 
complete a section in their group book on what situations make them happy and what situations they find stressful, and match a range of positive and negative emotions with physical responses. Students in PAL use a feeling-meter rather than a stressometer -where students are asked to label the intensity of their emotion rather than rating an emotion with a number. The stressometer was changed to a feeling-meter to encourage a balanced treatment of all types of emotions and to avoid rating different emotions that might convey that it is wrong to feel depressed or low. The 'feelings bingo' game also included a full range of positive emotions.

\section{Session Two: Practicing Gratitude}

The aim of session two is to increase students' awareness of the control they have over their happiness and the importance of gratitude in their lives. The session begins with a review of the previous session and the tasks practiced during the week. Team work is used to brainstorm and discuss the situations that make us feel happy or sad that are under our control as well as situations that are not under our control. Implications of control are discussed, including the option of gaining control by acknowledging that we can decide on what our response to a situation will be after our initial emotional reaction. The leader initiates a discussion on practicing gratitude and how it can be a helpful tool when things are not going well. For example, focusing on the things we are grateful for in a difficult situation can change our outlook and our mood. In groups of three or four, students share three things they are grateful for and then say why. Students then have a choice of writing a letter of thanks to someone they are grateful for, or decide on an activity they will do for someone to show their gratefulness towards them. ${ }^{10}$ The leader challenges students to test the theory of gratitude, i.e. to test if practicing gratitude will increase their happiness over the next week. The session ends with students being encouraged to write down three things they are grateful for each day, for at least seven consecutive days.

\section{Session Three: Identifying Strengths}

The aim of session three is to help students recognise character strengths in themselves and in others, and to identify how they can use their strengths. The session begins with a review of the previous session and tasks practiced during the week. Students pick a character they admire from a movie, a book, or real life, and they are asked to identify their

${ }^{10}$ Gratitude activities taken from Froh et al., 2008 and Toepher, 2011 
strengths and what they like about them. The activity above introduces the topic of the session and leads to introducing the character strengths listed in their group books, ${ }^{11}$ taken from the VIA (Peterson \& Seligman, 2004). Students rate each character strength, first by importance and then by what they admire in their friends the most. Students complete and score the character strength survey. ${ }^{12}$ The survey is followed by a discussion on what students think about the questions and if they agree with the results. The leader emphasises that the character strengths identified in the survey do not constitute an exhaustive list. In groups of three or four, students help each other think of ways they could use their strength in a difficult situation. Students write down in their group books three different ways in which they could use their top strength and where or when their strength might be particularly important. The session ends with students being encouraged to practice using their strength every day during the next week and to continue to think about what they are grateful for daily.

\section{Session Four: Enjoying Life}

The aim of session four is to be aware that savouring can increase happiness, to learn about flow, and to know how to increase the experience of flow. The session begins with a review of the previous session and of tasks practiced during the week. Students are introduced to savouring by slowly savouring a piece of chocolate and being aware of all the senses involved (perceptual sharpening). In pairs, students savour the past by recalling a memory and sharing it with each other. Students savour the future by thinking about an event they are looking forward to and discussing the event with others in the group. The group discusses what the advantages and disadvantages are of only ever savouring the past, for example a disadvantage can be missing the opportunity to enjoy what is happening now. ${ }^{13}$

The session then introduces the concept of flow as a state of being totally absorbed in the present. Flow is differentiated from savouring the present (flow is being immersed in an activity in the moment, whereas savouring the present is stepping outside the activity to review it). The leader explains how we experience flow and how we can create flow using the chart in the group book. ${ }^{14}$ In groups of three or four, students discuss times when they think

\footnotetext{
${ }^{11}$ See Appendix J: Character Strengths, From the PAL Group Book, p.16.

12 See Appendix K: Character Strength Survey, from PAL Group Book, p.18.

${ }^{13}$ Savouring activities taken from savouring research: Bijttebier et al., 2012; Bryant \& Veroff, 2007, Cafasso et al., 1994; Chadwick, 2012, Jose et al., 2012.

${ }^{14}$ Chart taken and adapted from Csikszentmihalyi,1997. See Appendix L: Flow Chart, From PAL Group Book, p.23.
} 
they have experienced flow. Students fill out a flow record in their group book, ${ }^{15}$ and this helps students identify what activities they experience the most flow in, so that they can choose to increase those activities. Students then discuss how they could change a monotonous activity into a more flow-producing activity. The session ends with students being encouraged to increase the activities wherein they experience flow and to pick a savouring activity to practice during the week. ${ }^{16}$

\section{Session Five: Problem Solving}

Session five is the same as the 'Problem Solving' session in Kiwi ACE except for the last section. In Kiwi ACE, the last section concerns the importance of rewarding yourself and having fun. Since these activities are too analogous to session four in PAL, this section was removed and replaced with 'changing places role play' (from session 3 in Kiwi ACE). In the 'changing places role play', students are encouraged to see a situation from another person's perspective.

There is a slight overlap of techniques between the programmes in session five. The "changing places role play" in PAL is very similar to a Cognitive-Behavioural technique. Likewise "the importance of having fun" in Kiwi ACE is very similar to a Positive Psychology technique. It would have been cleaner from a methodological point-of-view to take out both these sections. However, this overlap was allowed because 'the importance of having fun' in Kiwi ACE was included for a valid reason, and to remove it would mean tampering with the integrity of Kiwi ACE. Therefore "changing places" was included in PAL so that there would be a slight overlap in PAL also, with an attempt to create a balance.

\section{Session Six: Habits and Goal Setting}

The aim of session six is to increase awareness of how habits can influence mood and behaviour and to help students understand the importance of goal setting. Some of the activities in this session were taken and adapted from Kiwi ACE, the main difference between the sessions is PAL's focus on the importance of goal setting rather than changing 'bad' habits. ${ }^{17}$ The session begins with a review of the previous session and tasks practiced during the week. Students discuss what types of habits students have at their age, and what the immediate and delayed consequences are of each of these habits. The consequences are

\footnotetext{
${ }^{15}$ Flow record taken and adapted from Csikszentmihalyi,1997. See Appendix M: Flow Chart, From PAL Group Book, p.25.

${ }^{16}$ See Appendix N: Savouring Activities, From PAL Group Book, pp.21-22.

${ }^{17}$ The adaptations were based on 'goal-setting' research by Sheldon et al., 2002 and Wahl, 2011.
} 
written on the board and in groups of two, students use this information to list which habits they consider to be helpful and which they consider to be unhelpful. Both groups discuss the reasons for their answers.

This exercise is followed by a section on goal setting which is introduced as a helpful habit. Students discuss what goals they have set in the past, if they were successful, and why and why not. Students are asked what the rules should be for goal setting, and the leader incorporates these suggestions when introducing the rules set out in the facilitator's handbook. ${ }^{18}$ In pairs, students discuss what they want to happen in the next few months and throughout the year. Students decide on a goal and create an action plan in their group books. ${ }^{19}$ The session ends with students being encouraged to do the activity set out in their action plan for this week and to continue to practice the skills learnt in previous sessions.

\section{Session Seven: Building Relationships}

The aim of session seven is to increase awareness of the positive and long-term effects of acts of kindness and to develop students' social skills. The session begins with a review of the previous session and of tasks carried out during the week. The game 'alphabets' is followed by a discussion on kindness, in which students share their definition of kindness and how kindness can impact our lives. Students read through the list of acts of kindness ideas in their group book, ${ }^{20}$ then choose three small acts or one big act that they will carry out the following week. ${ }^{21}$ This exercise is followed by a section on social skills; this section was adapted from Kiwi ACE to be more compatible with PAL. The discussion on social skills does not look at 'good' and 'bad' social skills as does Kiwi ACE, but rather, takes the approach of person having more or less social skilfulness. The focus of the discussion is on building students' social skills by looking at what skills are most successful. Students picture someone they consider to have social skills and describe their behaviour. Students' ideas are put on the board and any skills that are not listed in their group book are added. The role plays remain the same as in Kiwi ACE: students discuss strategies for meeting new people and role play as a group or in pairs how they would deal with a difficult situation (such as meeting new people) using the skills and strategies discussed. The session ends with students

\footnotetext{
${ }^{18}$ See Appendix O: Goal Setting Rules, From PAL Facilitator's Handbook, p.51.

${ }^{19}$ See Appendix P: Goal Setting Action Plan, From PAL Group Book, p.31

${ }^{20}$ See Appendix Q: Acts of Kindness Ideas, From PAL Group Book, p.35.

${ }^{21}$ Kindness activities were taken and adapted from Lyubomirsky et al., 2005 and Lyubomirsky, \& Sheldon, 2008.
} 
being encouraged to carry out the acts of kindness they chose and to write down how they felt about it at the end of the day.

\section{Session Eight: Assertiveness}

Session eight is the same as the Assertiveness session in Kiwi ACE except for two sections. The section on 'why people may not be assertive' from Kiwi ACE is taken out from the PAL session as it examines the self talk and beliefs that can stop people from being assertive - this is not covered in PAL as it is based in Cognitive-Behavioural theory. To help students identify and address their own struggles and strengths in being assertive students in PAL are encouraged to discuss what they found to be difficult or easy about being assertive and why.

The other section that has been altered for PAL is the last section, the title from Kiwi ACE 'Handling difficult situations' has been relabelled 'Being assertive about who you are'. The scenarios were changed to be about students standing up for their opinions and ideas rather than just being assertive about a situation. For example, in Kiwi ACE one scenario is 'returning a faulty item to a shop'. In PAL a scenario is 'Some students in your class decide to do something to embarrass another student (who they think is not cool), however, you think that their idea is cruel and you don't want them to do it'.

\section{Session Nine: Negotiation and Compromise}

Session nine has the same content as the negotiation and compromise session in Kiwi $\mathrm{ACE}$ as there are no Cognitive-Behavioural techniques included in this session and it contains important social competence training.

\section{Session Ten: Liking who I am}

Session ten is similar to the self esteem session in Kiwi ACE, however the first section on core beliefs and realistic thinking was taken out as it is based on the CognitiveBehavioural approach. Beliefs that rob and boost self esteem were left in PAL because it was felt that the activities and content were too broad to be strictly cognitive behavioural. This session extends all the steps to boosting self esteem that are in Kiwi ACE, especially step one: respecting and caring for yourself. In PAL this step is expanded to include the mind, body and soul, and connecting with friends and family. It is PAL's aim to encourage the holistic view of well-being and that is including all aspects of a person's life. 


\section{Session Eleven: Review of PAL}

The aim of session eleven is to review the skills learnt in PAL. The session begins with reviewing the previous session and tasks practiced during the week. Students are divided into three groups and each group is allocated with a PAL Idea sheet. ${ }^{22}$ Each group is instructed to teach the rest of the group one PAL concept in any way they choose to, and students are encouraged to be as creative as they like. After these mini-lessons, students brainstorm possible future stresses that they may experience and some coping ideas to manage them. Students are provided with a list of contacts of the supports available if they need help with anything in the future. The session ends with the students sharing what they have gained from PAL and a farewell party is thrown for them by the leaders.

\section{Session Twelve: Survey and Review}

The session starts with a small discussion on how things are going for the students and what skills they have been practicing. Then students complete the survey they completed before the programme once more. ${ }^{23}$

\section{Participants}

All Year 9 students from nine local schools were screened with the CDI (Children's Depression Inventory, Kovacs, 1985, 2008). According to Kovacs (2008), a score of 10 for females and 11 for males on the CDI is considered to be the average score for a non-clinical sample years 13-17, and a score of 20 for females and 25 for males is indicative of depression. Individuals who score above average on the CDI are considered to be at-risk for developing depression later in life (Kovacs, 2008). Therefore females who scored between 10 and 19 and males who scored between 11 and 24 on the CDI from the eight schools were selected for the programme. All students in the programme were interviewed by the counsellor prior to commencement to ensure that they did not meet criteria for clinical depression. Students that met the clinical criteria for depression were not included, as the programmes are designed to prevent not treat depression.

A range of individuals were selected for each programme group with the aim of having an even spread of CDI scores (across the entire breadth of the mild-moderate range), a mix of ethnicities, and an even mix of genders (if they were in a co-ed group). The control group was created by matching individuals in the programme group by gender, CDI score

\footnotetext{
${ }^{22}$ See Appendix R: PAL Idea Sheet, From PAL Facilitator's Handbook, pp.84-86.

${ }^{23}$ To see a table of comparison between PAL and Kiwi ACE see Appendix S.
} 
and ethnicity with students from the same school.

Eight schools asked 12 of their 'at risk' students to participate in a programme group. Yalom (1995) suggests that an ideal group size is between 7 and 12. It was taken into consideration that a percentage of the students would decline, therefore 12 students were asked to participate in knowledge that the groups may be closer to 8-10 in size. Altogether 96 students were asked to be in one of the two programmes, but due to lack of consent and attrition only 65 students completed one of the two programmes (also some students across different schools never showed up to their programme and unfortunately these students were not replaced).

Twenty seven students completed the Kiwi ACE Programme and 38 completed PAL. In total 38 females and 27 males participated in a programme. The control group was composed of 69 students: 20 females and 49 males (matched control groups for both programmes were created to get more of a balance of gender and ethnicity, and match on scales at T1, see results section). A larger number of male control participants were obtained because a boys college in the Wellington region permitted us to use their Year 9s as a control group (but an intervention group was not run in the school due to logistical problems).

Below is a table showing the self reported ethnicities from the control and programme groups at time one:

Table 1. Self reported ethnicities of the control and programme groups

\begin{tabular}{lcc}
\hline Ethnicity & Control Group & Programme Group \\
\hline NZ European & 44 & 28 \\
Maori & 5 & 15 \\
Pacific Island & 8 & 10 \\
Asian & 5 & 4 \\
Other & 7 & 8 \\
Total & 69 & 65 \\
\hline
\end{tabular}

Nine schools in the wider Wellington region participated in the study. Four participated in the Kiwi ACE programme, four participated in the PAL, and one provided a control group only. The schools ranged from decile 3 to decile 10. Students from both public and private schools, religious and non-religious, single sex and co-ed schools participated. 
Due to the limited number and variety of schools, it was difficult to match the schools evenly for both programmes. Below is a table showing what type of schools participated in each programme:

Table 2. Gender, decile, school and year roll numbers and types of schools that participated in each programme

\begin{tabular}{|c|c|c|c|c|c|c|}
\hline & Gender & Decile & School roll & Year 9 role & Type & Programme \\
\hline 1 & Single Sex - Boys & 10 & 1527 & 270 & Public & Kiwi ACE \\
\hline 2 & Single Sex - Girls & 10 & 692 & 60 & Private & Kiwi ACE \\
\hline 3 & Single Sex- Girls & 8 & 960 & 200 & Public & Kiwi ACE \\
\hline 4 & Co-Ed & 3 & 271 & 54 & Catholic & Kiwi ACE \\
\hline 5 & Single Sex - Girls & 9 & 617 & 131 & Catholic & PAL \\
\hline 6 & Co-Ed & 10 & 1121 & 200 & Public & PAL \\
\hline 7 & Co-Ed & 5 & 388 & 80 & Catholic & PAL \\
\hline 8 & Co-Ed & 4 & 980 & 192 & Public & PAL \\
\hline 9 & Single Sex - Boys & 5 & 500 & 110 & Catholic & Control \\
\hline
\end{tabular}

\section{Ethical Procedure and Issues}

Ethics approval was obtained from SOPHEC (School of Psychology Human Ethics Committee) before any data collection occurred. Passive consent was used for the initial screening: a newsletter was sent out to all parents informing them that all Year 9 students would undertake a survey in the coming weeks. Passive consent for the screening was required as written consent would create the possibility that not all Year 9s would be screened and as a result our participants would not be a true representation of the school population. Psychological screening falls under the guidelines of normal and standard 
educational procedure, therefore passive consent was acceptable for these schools. Teachers made it clear to students that the survey was voluntary and that the information they provided in the questionnaires would only be seen by the school counsellor and the researcher.

Parental consent was obtained for participation in the programme and for the control groups to fill out the survey at three future time points. Students who yielded a high CDI score (above 19 for females and above 24 for males) were not included in the study as it was judged that it would be unethical to withhold notification to the school that these individuals were significantly at-risk. Also as mentioned earlier this programme was a prevention programme not a treatment programme for depression. Schools were notified of the few individuals who fell in the clinical range for depression.

The CDI contains a question on suicidal thought and intention, and includes other questions that can raise sensitive issues. Therefore, the surveys were scored within the week completed, and students who scored highly on the CDI or scored positive for suicide ideation were referred to the school counsellor for individual care (and they did not take part in the programme). Also the contact details of school counsellors and other local support people were provided on the information sheets given with the surveys so that individuals who scored below the cut-offs could seek help on their own.

The control group did not participate in any type of programme while the data collection was in process, however, they received normal care (and counselling if needed) from their school counsellors. A control group was necessary to ensure that these programmes were effective. A wait-list procedure could not be employed, whereby control participants receive the programme later because the programmes as the schools did not have the resources to do this. However, the control group individuals were given the opportunity to participate in an online resiliency programme after all data collection was completed.

\section{Procedure}

At the outset, all Year 9 students from each school completed a 126-item survey that incorporated eight different measures including the screening tool, i.e. the Children's Depression Inventory (CDI, Kovacs, 1985, 2008). (All measures are listed below in table 3). The survey took 35-40 minutes and was completed in class time. Students from each school who scored in the mild-to-moderate range on the CDI (10-19 for females, 11-23 for males) were selected into either a programme group or a control group (see participants in this method section for more details). The individuals who fell within the mild-to-moderate range on the CDI were invited to participate in the intervention programme that was made available 
to their particular school, and it was provided on-site during school hours. As there were only enough resources to have one programme per school, half of the schools participated in Kiwi ACE whilst the other half participated in PAL. Due to the limited number and variety of schools it was difficult to match the schools evenly for both programmes so they were selected at random. The programmes ran concurrently for 12 weeks, from July 2010 to October 2010, and both consisted of weekly one hour sessions during which a group of approximately 10 students met with the school counsellor and a clinical psychologist. The school counsellor was provided by the school and the clinical psychologist was external to the school and was provided by Gains Psychology (and funded by Compass Health). The psychologists were already familiar with the CBT techniques used in Kiwi ACE and were trained by the researcher in the theory and techniques used in PAL four weeks before the programme started. The counsellors were trained at the end of June 2010 by an external psychologist and the researcher in a two day course that covered every activity in each session, three weeks before the programmes commenced. The counsellors and psychologists had previous experience using CBT in a school setting and they spent extra time familiarising themselves with the positive psychology concepts from PAL.

At the end of twelve weeks, all control and programme participants were re-assessed with the same measures used at the initial screening. Further follow-up assessments were taken 6 months after the programme's finish (May, 2011) and one year after the programme's finish (October, 2011).

\section{Measures}

The CDI scores were used to screen individuals for the control and programme group, and also operated as the outcome for both programmes. Coping strategies, strengths and difficulties, resilience, and gratitude were assessed in order to measure potential mediators between the intervention programmes and the outcomes. Additionally happiness, well-being, and life satisfaction were assessed as potential outcomes for the PAL.

The survey included eight different scales that are listed below in the order that they were presented in the survey (the Cronbach's alphas presented are from the present study):

The Strengths and Difficulties Survey (SDQ) has 25 items including: 'I am kind to younger children' 'I worry a lot' and 'I cannot stay still for long' (Goodman, 2001). The survey asks respondents to give their answers on the basis of how things have been over the last six months and to mark a box for Not True, Somewhat True or Certainly true. The SDQ measures pro-social behaviour and difficulties including: emotional symptoms, conduct 
problems, hyperactivity-inattention and peer problems. The highest possible score for experiencing difficulties is 40 and a score of 16-19 is considered borderline, a score of 20 and over is considered 'abnormal' (Goodman, 2001). In the current study Total Difficulties obtained an acceptable level of internal consistency $(\alpha=.70)$. However, only two subscales obtained acceptable levels of internal consistency, these were: emotional symptoms $(\alpha=.72)$ and peer problems $(\alpha=.71)$.

The Children's Depression Inventory (CDI) has 27 items and asks respondents to pick one sentence from each group that best describes the way they have been feeling for the last two weeks, for example; I am sad once in a while, I am sad many times, I am sad all the time (Kovacs, 1985, 2008). A score from 10-24 indicates that the respondent is showing some symptoms of depression and is at-risk of developing depression later in life, a score above 24 is regarded as clinical depression however a thorough interview is needed to determine this. The CDI also has five dimensions, all the dimensions obtained acceptable levels of internal consistency; Negative mood $(\alpha=.71)$ Interpersonal problems $(\alpha=.61)$, Ineffectiveness ( $\alpha=.69)$, Anhedonia $(\alpha=.73)$ and Negative self esteem $(\alpha=.73)$, the total scale also obtained a satisfactory level internal consistency as can be seen in table 3 .

The Resilience Scale has 14 items including 'I usually manage one way or another' and 'I am determined' (RS-14) (Wagnild \& Young, 1993). Responses are indicated on a 6point Likert scale, with responses ranging from strongly disagree to strongly agree. A score from 14-56 is considered very low, 57- 64 is considered low, 65-73 is on the low end, $74-$ 81 is moderate, $82-90$ is medium high and $91-98$ is considered high. The dimensions of resilience include: self-reliance, meaningfulness, equanimity, perseverance, and existential aloneness. In this present study only self reliance $(\alpha=.77)$ and meaningfulness $(\alpha=.75)$ obtained acceptable levels of internal consistency, however the total scale obtained a satisfactory level internal consistency as can be seen in table 3 .

The Children's Integrated Stress and Coping Scale has 21 items including 'I talked to someone to feel better' and 'I ignored or tried to get away from the problem' (Jose \& Huntsinger, 2005). The questionnaire asks respondents to write down a stressful event that they experienced in the last month and then to write a number on the left side to show how much you used these ways of dealing with the problem, the numbers range from $0=$ not at all to $3=$ a lot. Nine items loaded onto adaptive coping $(10,11,12,13,14,16,17,20,21)$ and nine items loaded onto maladaptive coping $(2,4,5,6,7,9,15,18,19)$, three items did not load 
onto either scale. Both adaptive and maladaptive coping obtained acceptable levels of internal consistency as can be seen on table 3 .

The Ryff's Psychological well-being Scales has 24 items including 'In general I feel positive and confident about myself' and reverse coded items including 'I don't have many people who want to listen when I need to talk' (RPWS) (Ryff \& Keyes, 1995). Responses are indicated on a 6-point Likert scale, with responses ranging from strongly disagree to strongly agree, higher scores are indicative of higher levels of psychological well-being. The dimensions of well-being include: self-acceptance, positive relations with others, autonomy, environmental mastery, purpose in life, and personal growth. Only environmental mastery $(\alpha=.69)$, Self acceptance $(\alpha=.73)$, and positive relationships $(\alpha=.67)$ obtained an acceptable (or close to acceptable) levels of internal consistency. This was likely due to the small number of items loading onto each subscale, as only four items per subscale were used and the RPWS is most commonly used with at least seven items and sometimes as much as 20 items loading onto each subscale. The original questionnaire included 120 items (20 per dimension) but shorter versions comprising 84 items (14 per dimension), 54 items ( 9 per dimension), 42 items (7 per dimension), 24 items (4 per dimension) and 18 items ( 3 per dimension) are now widely used. The 120 or 84 item RPWS could not be used in the present study as it would have made the total survey too long. It is quite common for studies that use short measures with Ryffs constructs to typically obtain low internal consistencies for the sub-scales (Cheng, \& Chan, 2005; Ryff \& Keyes, 1995; Van Dierendonck, 2004), however, the 24-item RPWS has been successfully used (Seaton, Scottham, \& Sellers, 2006). The total RPWS in this study did obtain a satisfactory level of internal consistency as can be seen in table 3 .

The Subjective Happiness Scale (SHS) has four items, including 'in general I consider myself: $1=$ 'not a very happy person' to $7=$ 'a very happy person' (Lyubomirsky $\&$ Lepper, 1999). Responses are indicated on a 7-point likert scale and are specifically tailored to each question as can be seen in the example just given. Higher scores are indicative of higher levels of happiness. In this present study the last item of the SHS was taken out of the total score after a reliability analysis showed that the cronbachs alpha would significantly increase once it was removed.

The Gratitude Survey (GQ) has six items including 'I have so much in life to be thankful for' and 'I am grateful to a wide variety of people' (McCullough, Emmons, \& Tsang, 2002). The respondent must answer each item with a number that corresponds to the 
answer given at the top of the page, ranging from $1=$ strongly disagree to $7=$ strongly agree. Higher scores are indicative of higher levels of gratitude. In this present study the last item of the GQ was taken out of the total score after a reliability analysis showed that the Cronbachs alpha would significantly increase once it was removed.

The Satisfaction with Life Scale has five items including 'In most ways my life is close to ideal' and 'I am satisfied with life' (Diener, Emmons, Larsen \& Griffin, 1985). The respondent must answer each item with a number that corresponds to the answer given at the top of the page, ranging from $1=$ strongly disagree to $7=$ strongly agree. Individuals who score from $5-9$ are considered to be extremely dissatisfied, 10 - 14 dissatisfied, $15-19$ slightly below average, $20-24$ is an average score, $25-29$ is a high score and $30-35$ is a very high score, these individuals are considered to be highly satisfied with their life. Below is a table showing each scale that was included in the survey and its relevant components: 
Table 3. The Scales in the Survey and their Author, Number of Items, Cronbach's Alpha from this study and Purpose in the Study

\begin{tabular}{|c|c|c|c|c|}
\hline Name of scale & Author(s) & $\begin{array}{l}\text { No. of } \\
\text { Items }\end{array}$ & Cronbach's alpha & Purpose of scale \\
\hline $\begin{array}{l}\text { The Children's Depression } \\
\text { Inventory }\end{array}$ & Kovacs, 1985 & 27 & 90 & $\begin{array}{l}\text { Screening and } \\
\text { Outcome }\end{array}$ \\
\hline $\begin{array}{l}\text { The Strengths and } \\
\text { Difficulties Survey }\end{array}$ & Goodman, 2001 & 25 & .70 & Possible Mediator \\
\hline \multirow{2}{*}{$\begin{array}{l}\text { The Children's Integrated } \\
\text { Stress and Coping Scale }\end{array}$} & Jose \& Huntsinger, 2005 & 9 & Adaptive Coping .76 & Possible Mediator \\
\hline & & 9 & Maladaptive Coping .70 & Possible Mediator \\
\hline $\begin{array}{l}\text { Ryff's Psychological well- } \\
\text { being Scales }\end{array}$ & Ryff \& Keyes, 1995 & 24 & .86 & Possible Outcome \\
\hline Subjective Happiness Scale & $\begin{array}{l}\text { Lyubomirsky \& Lepper, } \\
1999\end{array}$ & 4 & .85 & $\begin{array}{l}\text { Possible Outcome \& } \\
\text { Mediator }\end{array}$ \\
\hline $\begin{array}{l}\text { The Satisfaction with Life } \\
\text { Scale }\end{array}$ & Diener, 1991 & 5 & .85 & $\begin{array}{l}\text { Possible Outcome \& } \\
\text { Mediator }\end{array}$ \\
\hline The Resilience Scale & Wagnild \& Young, 1993 & 14 & .90 & $\begin{array}{l}\text { Possible Outcome \& } \\
\text { Mediator }\end{array}$ \\
\hline Gratitude Survey & $\begin{array}{l}\text { McCullough, Emmons, } \\
\& \text { Tsang, } 2002\end{array}$ & 6 & .81 & Possible Mediator \\
\hline
\end{tabular}

\section{Qualitative Methodology}

On the twelfth and final session of the programme, each student filled out a feedback form and participated in a discussion on how the programme went for them. Students were encouraged to be completely honest, as the programme was being evaluated and their input would help to improve the programme for students participating in the future. A relaxed atmosphere was created for the discussion: no desks, and pizza and chocolate were provided for everyone to share. The session began with the leaders thanking and congratulating the 
students on finishing the programme. Students were asked to complete a short feedback form $^{24}$ and then they verbally discussed their impression of the programme with the group and the leader. After the discussion, completion certificates were awarded to each student.

The leaders of each group, both the psychologist and school counsellor, also completed feedback forms. ${ }^{25}$ A discussion was held with each leader separately and the researcher to provide an opportunity for each of them to raise any issues/events that occurred during the programme or between students or leaders, which may have impacted on the quality of the programme. The aim of the feedback questionnaire was to collect students' thoughts and feelings about the programme and determine if there was any stigma associated with participating in the programme. Also the questionnaire was designed to help gauge how each session went for the students and leaders in both process and content.

The feedback questionnaire included open-ended questions asking why the students joined the group, how they felt about it and how their peers responded. The form also asked students to rate each session from the programme on a 7-point Likert scale to indicate how much they enjoyed each session, how helpful they found it, if they still practice the skills learnt, and how much effort they put in to the session. The last section used a 7-point Likert scale and open-ended questions to explore how well students got on with each other and their leaders, if there was anyone who distracted them in the group, and how they found the programme overall. The leader's feedback form asked the leaders to rate each session of the programme on a 7-point Likert scale to indicate how much they thought the students enjoyed the session, how helpful they found it, and if they noticed the students using the skills taught. The last section used a 7-point Likert scale and open-ended questions to explore the leader's perspective on how well they thought students got on with each other and themselves, if there was anyone who distracted the students in the group, and how they found the programme overall. In addition to the feedback forms, the leaders also filled out a checklist and made relevant comments in their handbook after each session as part of the programme.

\footnotetext{
${ }^{24}$ See Appendix T, on CD: Kiwi ACE Student Feedback Form; See Appendix U on CD: PAL Student Feedback Form.

${ }^{25}$ See Appendix V, on CD: Kiwi ACE Leader Feedback Form; See Appendix W on CD: PAL Leader Feedback Form.
} 


\section{Chapter 3: Results}

\section{Preliminary Results}

Descriptive Statistics at T1 before Control and Programme Groups were Selected

At time one (T1) 1,171 participants from 9 schools were screened prior to selection of the control and programme groups. Correlations between all scales at T1 were calculated and all correlations were shown to be statistically significant (see Table 4). The means, standard deviations and percentages were calculated from $\mathrm{T} 1$ for all the scales and subscales in the well-being, mood and coping survey. The scores reported are compared to New Zealand or international norms where possible.

The mean for difficulties $(M=9.85, S D=5.65)$ from the Strengths and Difficulties Questionnaire fell in the New Zealand normative data range $(M=10.20, S D=5.00)$ (Black, Pulford, Christie, \& Wheeler, 2010). Ten percent of the participants scored in the abnormal range for difficulties, and 6\% scored in the borderline range. The means for the subscales of the Strengths and Difficulties Questionnaire were calculated and shown to fall in the low range (scores varied from 1 to 10$)$ : emotional symptoms $(M=2.54, S D=2.08)$, conduct problems $(M=1.89, S D=1.73)$, hyperactivity-inattention $(M=3.75, S D=2.26)$, peer problems $(M=1.67, S D=1.66)$, and subjective pro-social behaviour $(M=7.42, S D=1.75)$, these means all fall in the New Zealand normative data range ${ }^{26}$ (Black et al., 2010).

\footnotetext{
${ }^{26} \mathrm{NZ}$ norms from Black (2010): emotional symptoms $(M=2.7, S D=2.1)$, conduct problems $(M=2.1, S D=1.6)$, hyperactivity-inattention $(M=3.7, S D=2.2)$, peer problems $(M=1.7, S D=1.5)$, and subjective pro-social behaviour $(M=7.6, S D=1.6)$.
} 
Table 4. Correlations of Difficulties, Depression, Resilience, Well-being, Happiness, Gratitude, Satisfaction with Life, Adaptive Coping and Maladaptive Coping

\begin{tabular}{|c|c|c|c|c|c|c|c|c|}
\hline & Difficulties & Depression & Resilience & Well-being & Happiness & Gratitude & $\begin{array}{c}\text { Life } \\
\text { satisfaction }\end{array}$ & $\begin{array}{c}\text { Adaptive } \\
\text { coping }\end{array}$ \\
\hline Difficulties & & & & & & & & \\
\hline Depression & $.76 * *$ & & & & & & & \\
\hline Resilience & $-.62 * *$ & $-.71 * *$ & & & & & & \\
\hline Well-being & $-.69 * *$ & $-.73 * *$ & $.74 * *$ & & & & & \\
\hline Happiness & $-.49 * *$ & $-.62 * *$ & $.62 * *$ & $.63 * *$ & & & & \\
\hline Gratitude & $-.54 * *$ & $-.62 * *$ & $.66 * *$ & $.66^{* *}$ & $.59 * *$ & & & \\
\hline Life satisfaction & $-.55^{* *}$ & $-.68 * *$ & $.68 * *$ & $.67 * *$ & $.69 * *$ & $.69 * *$ & & \\
\hline Adaptive coping & $-.28 * *$ & $-.34 * *$ & $.38 * *$ & $.36 * *$ & $.29 * *$ & $.37 * *$ & $.36 * *$ & \\
\hline Maladaptive coping & $.56 * *$ & $.60 * *$ & $-.43 * *$ & $-.51 * *$ & $-.35 * *$ & $-.41 * *$ & $-.41 * *$ & $-.09 * *$ \\
\hline
\end{tabular}

$* * p<0.01 ; \mathrm{N}=1174$ 
The mean score from the Resilience Scale $(M=74.81, S D=13.01)$ fell in the moderate to high range for resilience (Moderate to high $=74-81$, Wagnild \& Young, 1993). Nine percent of participants scored high in resilience, $50 \%$ of participants scored moderateto-moderately high in resilience, $32 \%$ scored low-to-very low, and $9 \%$ scored very low. The means for the two subscales showed reasonably high levels of both self reliance $(M=26.00$, $\mathrm{SD}=4.85)$ and meaningfulness $(\mathrm{M}=17.14, \mathrm{SD}=3.34)$ (only these two subscales were used as they were the only two that reached acceptable levels of internal consistency, see method section p.95).

The mean scores were calculated for adaptive coping $(M=1.56, S D=.59)$ and maladaptive coping $(M=.78, S D=.49)$ from the Children's Integrated Stress and Coping Scale (CISCS) (Jose \& Huntsinger, 2005). The mean for Adaptive coping was slightly higher than the American normative data range $(M=1.20, S D=.49)$ and maladaptive coping was lower than the American normative data range $(M=1.24, S D=.46)$ (Jose \& Huntsinger, 2005).

The mean was calculated for the overall Ryff's Psychological Well-being Scale $(M=$ 103.18, $S D=16.12)$ and the three subscales: environmental mastery $(M=17.07, S D=3.87)$, self acceptance $(M=17.08, S D=4.02)$, and positive relationships $(M=19.32, S D=3.72)$ (as these three were the only subscales that obtained acceptable levels of internal consistency, see method section p.96). Less than one percent of participants obtained a very low score on well-being, $2.7 \%$ scored moderately low, $32.2 \%$ scored in the average range, $49.6 \%$ scored medium high, and $15.4 \%$ scored in the very high range .

The mean score for the Subjective Happiness Scale (SHS) was calculated ( $M=15.72$, $S D=3.40$ ) and a frequency analysis showed that $2.4 \%$ of the students considered themselves as not a very happy person, $44.2 \%$ considered themselves as a very happy person and $53.4 \%$ considered themselves as somewhere in between.

The mean score for the gratitude was calculated $(M=29.02, S D=5.26)$ and a frequency analysis revealed that $1.2 \%$ of participants obtained a low score in gratitude, $31.3 \%$ scored in the average range, and $67.5 \%$ obtained a high score in gratitude. The mean for Gratitude fell in the normative data range $(M=28.87, S D=8.26)$ (Wood, et al., 2008).

The mean for the Satisfaction With Life Scale $(M=26.16, S D=6.17)$ was slightly higher than American norms $(M=23.5, S D=6.43)$ (Pavot \& Diener, 2008). Eighteen percent of participants had an average score in life satisfaction, $67.5 \%$ had a high score or a very high score in life satisfaction, $12.5 \%$ had a score below average, and $2 \%$ of the sample received scores that suggested that they were extremely dissatisfied with their life. 
The mean for depression $(M=8.15, S D=7.28)$ from the Children's Depression Inventory (CDI) was slightly lower than the normative range for adolescents $(M=9.98, S D=$ 7.29) (Kovacs, 2008). The means for the subscales were also calculated; negative mood $(M=$ $1.78, S D=1.93)$, interpersonal problems $(M=.56, S D=1.01)$, ineffectiveness $(M=1.40$, $S D=1.66)$, anhedonia $(M=2.49, S D=2.52)$, and negative self esteem $(M=1.27, S D=$ 1.65). A frequency analysis revealed that $4.3 \%$ of participants could be placed in the clinical range for depression, and $23.1 \%$ of participants fell in the mild-to-moderate range and therefore potentially at risk for developing depression later in life.

\section{Selection of Programme and Control Groups}

The CDI was used as the screening tool to gather a sample of "at risk" students to invite to be part of the control or programme group. As shown above: $23.1 \%$ of participants scored in the mild-to-moderate range in depression, constituting 231 participants from the original sample of 1171. From this sample, 96 students were asked to be in a programme group (12 students from each school), and after attrition and lack of consent, 65 students completed a programme (27 students completed Kiwi ACE and 38 completed PAL).

The control group was initially selected by matching gender, ethnicity, and depression score of programme participants from each school. Control participants had to complete the questionnaire at screening and at one later time point to be included in the final control group. Also participants that clearly did not answer the questionnaire seriously (i.e. circling the same number throughout the questionnaire) were removed.

\section{Differences in Depression Scores between and within Schools}

CDI means were compared between groups within schools and between schools to determine if a participant's school had an impact on their depression score and to ensure all groups were adequately matched at T1. No significant differences in depression scores were found between the control and programme groups within schools (see Table 5). There were no significant differences between control groups from the different schools $(F(6,62)=.61, p$ $=.72$, partial $\eta 2=.06$ ). There were also no significant differences in depression scores between programme groups $(F(1,63)=.35, p=.56$, partial $\eta 2=.01)$.

\section{Multilevel Analysis of Variance by School}

In addition to the above analyses, a multilevel analysis was run using hierarchical linear modelling to confirm that a participant's depression scores did not systematically vary by nesting within school. HLM analysis looks at the variance by school and determines 
whether there is significant poolings of variance of depression scores by school. The obtained result was non-significant, $\mathrm{ICC}=.001, p=.30$; since the intraclass correlation was small and non-significant, one can conclude that initial depression scores did not significantly vary by school.

\section{Missing Data}

At time 2 there were no participants missing in the data collection. At time 3 there was 1 participant missing from Kiwi ACE, 1 from PAL and 2 from the control group. At time 4 there were 14 participants missing from the sample, 2 from Kiwi ACE, 4 from PAL and 8 from the control group.

Missing data was very low at all time points. At time 1 there was half a percent of missing items $(.005 \%)$, at time 2 there was $.006 \%$ missing items, at time 3 there was $.007 \%$ missing items and at time 4 there was $.002 \%$ missing items. All missing data was replaced using the expectation-maximization (EM) algorithm (Dempster, Laird, \& Rubin, 1977). The EM algorithm is a validated approach that derives maximum likelihood estimates in parametric models for incomplete data. This method was used for all scales at T1. Then EM was used on PAL, Kiwi ACE, and the control group separately across T2-T3 in order to be consistent in the treatment of missingness across the groups.

\section{Means and Standard Deviations of the Control Group at all Time Points}

The matched control groups were selected at the last time point (Time 4) in order to have sufficient numbers of robustly matched control participants who yielded data across all 4 time points. Attrition would have caused significant loss of information. The means and standard deviations for the control group at all time points were calculated (please see Table $6)$. 
Table 5. Depression score differences between control and programme groups in each school

\begin{tabular}{|c|c|c|c|c|c|c|}
\hline School & Group & Mean & SD & $t$ & $\mathrm{df}$ & $p$ \\
\hline \multirow[t]{2}{*}{1} & Control & 14.64 & 1.75 & \multirow{2}{*}{0.73} & \multirow{2}{*}{18} & \multirow{2}{*}{0.47} \\
\hline & Programme & 15.49 & 3.25 & & & \\
\hline \multirow[t]{2}{*}{2} & Control & 14.47 & 4.10 & \multirow{2}{*}{-.05} & \multirow{2}{*}{21} & \multirow{2}{*}{0.96} \\
\hline & Programme & 14.38 & 4.07 & & & \\
\hline \multirow[t]{2}{*}{3} & Control & 13.10 & 3.77 & \multirow{2}{*}{0.17} & \multirow{2}{*}{11} & \multirow{2}{*}{0.87} \\
\hline & Programme & 13.50 & 3.47 & & & \\
\hline \multirow[t]{2}{*}{4} & \multicolumn{3}{|l|}{ No control group } & & & \\
\hline & Programme & 13.00 & 5.02 & & & \\
\hline \multirow[t]{2}{*}{5} & Control & 11.75 & 2.50 & \multirow{2}{*}{0.99} & \multirow{2}{*}{12} & \multirow{2}{*}{0.34} \\
\hline & Programme & 14.15 & 4.47 & & & \\
\hline \multirow[t]{2}{*}{6} & Control & 13.44 & 4.48 & \multirow{2}{*}{-0.11} & \multirow{2}{*}{12} & \multirow{2}{*}{0.91} \\
\hline & Programme & 13.20 & 2.39 & & & \\
\hline \multirow[t]{2}{*}{7} & Control & 15.28 & 4.27 & \multirow{2}{*}{0.64} & \multirow{2}{*}{26} & \multirow{2}{*}{0.53} \\
\hline & Programme & 16.49 & 5.72 & & & \\
\hline \multirow[t]{2}{*}{8} & No control group & & & & & \\
\hline & Programme & 15.69 & 3.77 & & & \\
\hline 9 & Control group only & 14.55 & 4.14 & & & \\
\hline
\end{tabular}


Table 6. Control group T1-T4 Means and Standard Deviations of Difficulties, Depression, Resilience, Well-being, Gratitude,

Happiness, Satisfaction with Life, Adaptive Coping, and Maladaptive Coping

\begin{tabular}{|c|c|c|c|c|c|c|c|c|c|}
\hline & Difficulties & Depression & Resilience & Well-being & Gratitude & Happiness & $\begin{array}{c}\text { Life } \\
\text { satisfaction }\end{array}$ & $\begin{array}{c}\text { Adaptive } \\
\text { coping }\end{array}$ & $\begin{array}{c}\text { Maladaptive } \\
\text { coping }\end{array}$ \\
\hline T1 Mean & 14.12 & 14.46 & 65.25 & 90.58 & 26.78 & 13.32 & 22.28 & 13.39 & 9.12 \\
\hline T1 Standard deviation & 4.67 & 4.04 & 11.69 & 11.36 & 5.75 & 3.96 & 6.72 & 5.51 & 3.92 \\
\hline T2 Standard deviation & 4.82 & 6.82 & 13.60 & 12.90 & 5.77 & 3.73 & 5.73 & 5.19 & 3.86 \\
\hline T3 Mean & 13.38 & 13.24 & 66.51 & 92.75 & 26.24 & 13.28 & 21.61 & 12.73 & 9.13 \\
\hline T3 Standard deviation & 5.74 & 7.23 & 12.67 & 12.92 & 6.01 & 3.69 & 6.15 & 5.39 & 4.08 \\
\hline
\end{tabular}

$\mathrm{N}=69$ 


\section{Propensity Score Matching}

After T4 the control groups for both programmes were matched by using propensity score matching to ensure that students were matched on their demographic variables as well as on their scores from all eight scales in the survey at T1. Propensity score matching helps to reduce confounding variance and selection effects that are generally problematic in datasets collected through subject-variable studies or even in random assignment studies (Roseburn \& Rubin, 1983). With propensity score matching it is possible to make sub-samples of similar individuals that are only differentiated by their membership to a control or treatment group, ensuring that any obtained mean differences between the treatment and control group are due predominately to their membership in the treatment or control group.

\section{Calculating the Propensity Scores and Matching}

Each programme group obtained their own matched control group through propensity score matching since individuals in the Kiwi ACE and PAL groups were not matched to each other on all variables at T1 (they were initially matched only with depression scores, gender, and ethnicity). Propensity score matching with replacement was used (rather than without replacement), meaning each control participant could be matched more than once to a different treatment participant. Although this approach has the disadvantage of reducing variance, it has the advantage of increasing the average quality (closeness) of matching and thus decreasing the bias between treatment and control groups, and is a widely recognised method for propensity score matching (Caliendo \& Kopeinig, 2005; Dehejia \& Wahba, 2002; Hill, Waldfogel, Brooks-Gunn, \& Han, 2005).

A logistic regression was used to calculate the propensity score for each participant from both groups, with the nine total scale scores entered as quantitative predictors (difficulties, depression, resilience, well-being, happiness, gratitude, satisfaction with life, adaptive and maladaptive coping). School decile was not included in the equation as it was not correlated with any other variables. The propensity score was calculated without gender and ethnicity as categorical variables since they were used to aid initial manual matching.

The matching process was performed using the propensity scores, gender and ethnicity to match programme participants with control participants. The matching criterion was a delta no larger than .10 , if no match occurred in this delta range then the smallest delta took priority. 
Matching Results for Kiwi ACE and PAL

After matching with replacement, 30 control participants were matched to the 38 participants in PAL, and separately 25 control participants were matched to the 28 participants in Kiwi ACE. To test that both programmes groups had been matched adequately and that there were no differences at T1, a MANOVA analysis was run with all eight variables comparing Kiwi ACE and its matched control group at T1 and a MANOVA was run with all eight variables comparing PAL and its matched group at T1. No significant differences in any of the eight scale scores between Kiwi ACE and its matched control group (see table 7) were found, and no significant differences between PAL and its matched control group (see table 8) were found.

A chi-square analysis was run to ensure there were no significant differences with gender and ethnicity for both matched groups. The results showed that there were no significant differences between Kiwi ACE and its matched control group in gender $(p=.79)$ or ethnicity $(p>.43)$. There were also no significant differences between PAL and its matched control group in gender $(p=.45)$ or ethnicity $(p>.68)$. As can be seen from the tables below (Kiwi ACE table 9, PAL table 10), matching helped bring a closer balance of gender and ethnicity to the control groups. 
Table 7. Control and Kiwi ACE means, standard deviations, F score, significance and effect size in differences at T1 for Difficulties, Depression, Resilience, Well-being, Happiness,

Gratitude, Satisfaction with Life, Adaptive Coping and Maladaptive Coping

\begin{tabular}{|c|c|c|c|c|c|c|}
\hline & Group & Mean & $\begin{array}{c}\text { Std. } \\
\text { Deviation }\end{array}$ & $\mathrm{F}$ & $p$ & $\eta 2$ \\
\hline \multirow[t]{2}{*}{ Difficulties } & Control & 15.16 & 5.30 & \multirow{2}{*}{0.02} & \multirow{2}{*}{0.88} & \multirow{2}{*}{0.00} \\
\hline & Kiwi ACE & 15.37 & 4.58 & & & \\
\hline \multirow[t]{2}{*}{ Depression } & Control & 15.06 & 4.47 & \multirow{2}{*}{0.00} & \multirow{2}{*}{0.97} & \multirow{2}{*}{0.00} \\
\hline & Kiwi ACE & 15.02 & 4.96 & & & \\
\hline \multirow[t]{2}{*}{ Resilience } & Control & 62.63 & 13.69 & \multirow{2}{*}{0.03} & \multirow{2}{*}{0.87} & \multirow{2}{*}{0.00} \\
\hline & Kiwi ACE & 63.22 & 12.84 & & & \\
\hline \multirow[t]{2}{*}{ Well-being } & Control & 87.20 & 11.49 & \multirow{2}{*}{0.45} & \multirow{2}{*}{0.51} & \multirow{2}{*}{0.01} \\
\hline & Kiwi ACE & 89.30 & 11.11 & & & \\
\hline \multirow[t]{2}{*}{ Happiness } & Control & 11.97 & 4.11 & \multirow{2}{*}{0.14} & \multirow{2}{*}{0.71} & \multirow{2}{*}{0.00} \\
\hline & Kiwi ACE & 12.36 & 3.32 & & & \\
\hline \multirow[t]{2}{*}{ Gratitude } & Control & 25.83 & 6.79 & \multirow{2}{*}{0.02} & \multirow{2}{*}{0.89} & \multirow{2}{*}{0.00} \\
\hline & Kiwi ACE & 26.05 & 4.93 & & & \\
\hline \multirow{2}{*}{$\begin{array}{l}\text { Satisfaction with } \\
\text { life }\end{array}$} & Control & 20.29 & 6.97 & \multirow{2}{*}{0.02} & \multirow{2}{*}{0.90} & \multirow{2}{*}{0.00} \\
\hline & Kiwi ACE & 20.07 & 5.40 & & & \\
\hline \multirow[t]{2}{*}{ Adaptive coping } & Control & 14.41 & 6.09 & \multirow{2}{*}{1.28} & \multirow{2}{*}{0.26} & \multirow{2}{*}{0.02} \\
\hline & Kiwi ACE & 12.60 & 5.44 & & & \\
\hline \multirow{2}{*}{$\begin{array}{l}\text { Maladaptive } \\
\text { coping }\end{array}$} & Control & 9.53 & 3.55 & \multirow{2}{*}{0.12} & \multirow{2}{*}{0.74} & \multirow{2}{*}{0.00} \\
\hline & Kiwi ACE & 9.95 & 5.11 & & & \\
\hline
\end{tabular}


Table 8. Control and PAL means, standard deviations, F score, significance and effect size in differences at T1 for Difficulties, Depression, Resilience, Well-being, Happiness, Gratitude, Satisfaction with Life, Adaptive Coping and Maladaptive Coping

\begin{tabular}{|c|c|c|c|c|c|c|}
\hline & Group & Mean & $\begin{array}{c}\text { Std. } \\
\text { Deviation }\end{array}$ & $\mathrm{F}$ & Sig & $\eta 2$ \\
\hline \multirow[t]{2}{*}{ Difficulties } & Control & 14.13 & 5.39 & \multirow{2}{*}{0.00} & \multirow{2}{*}{0.98} & \multirow{2}{*}{0.00} \\
\hline & PAL & 14.16 & 4.42 & & & \\
\hline \multirow[t]{2}{*}{ Depression } & Control & 14.51 & 4.73 & \multirow{2}{*}{0.02} & \multirow{2}{*}{0.90} & \multirow{2}{*}{0.00} \\
\hline & PAL & 14.38 & 3.75 & & & \\
\hline \multirow[t]{2}{*}{ resilience } & Control & 64.93 & 14.14 & \multirow{2}{*}{0.92} & \multirow{2}{*}{0.34} & \multirow{2}{*}{0.01} \\
\hline & PAL & 68.00 & 12.20 & & & \\
\hline \multirow[t]{2}{*}{ Well-being } & Control & 91.40 & 11.55 & \multirow{2}{*}{0.07} & \multirow{2}{*}{0.80} & \multirow{2}{*}{0.00} \\
\hline & PAL & 92.05 & 8.89 & & & \\
\hline \multirow[t]{2}{*}{ Happiness } & Control & 13.59 & 4.82 & \multirow{2}{*}{0.03} & \multirow{2}{*}{0.87} & \multirow{2}{*}{0.00} \\
\hline & PAL & 13.74 & 3.30 & & & \\
\hline \multirow[t]{2}{*}{ Gratitude } & Control & 26.84 & 6.67 & \multirow{2}{*}{0.00} & \multirow{2}{*}{0.95} & \multirow{2}{*}{0.00} \\
\hline & PAL & 26.75 & 5.04 & & & \\
\hline \multirow[t]{2}{*}{ Life satisfaction } & Control & 22.05 & 8.20 & \multirow{2}{*}{0.00} & \multirow{2}{*}{1.00} & \multirow{2}{*}{0.00} \\
\hline & PAL & 22.04 & 6.30 & & & \\
\hline \multirow[t]{2}{*}{ Adaptive coping } & Control & 12.73 & 6.89 & \multirow{2}{*}{2.13} & \multirow{2}{*}{0.15} & \multirow{2}{*}{0.03} \\
\hline & PAL & 10.51 & 5.70 & & & \\
\hline \multirow{2}{*}{$\begin{array}{l}\text { Maladaptive } \\
\text { coping }\end{array}$} & Control & 9.87 & 4.84 & \multirow{2}{*}{0.36} & \multirow{2}{*}{0.55} & \multirow{2}{*}{0.01} \\
\hline & PAL & 10.49 & 3.82 & & & \\
\hline
\end{tabular}


Table 9. Comparing number of male, female and ethnicity (NZ European, Māori, Pacific Islander, Asian and other ethnicities) in Kiwi ACE, Matched control and Unmatched Control

\begin{tabular}{lccccccccc}
\hline & \multicolumn{3}{c}{ Kiwi ACE } & \multicolumn{3}{c}{ Matched Control } & \multicolumn{2}{c}{ Unmatched Control } \\
\hline Ethnicity & Female & Male & Total & Female & Male & Total & Female & Male & Total \\
\hline NZ European & 5 & 7 & 12 & 8 & 8 & 16 & 12 & 32 & 44 \\
Māori & 3 & 2 & 5 & 2 & 1 & 3 & 3 & 2 & 5 \\
Pacific Islander & 2 & 1 & 3 & 1 & 1 & 2 & 4 & 4 & 8 \\
Asian & 1 & 1 & 2 & 0 & 2 & 2 & 0 & 5 & 5 \\
Other & 2 & 3 & 5 & 0 & 2 & 2 & 1 & 6 & 7 \\
\hline Totals & 13 & 14 & 27 & 11 & 14 & 25 & 20 & 49 & 69
\end{tabular}

Table 10. Comparing number of male, female and ethnicity (NZ European, Māori, Pacific Islander, Asian and other ethnicities) in PAL, matched control and unmatched control

\begin{tabular}{lccccccccc}
\hline & \multicolumn{3}{c}{ PAL } & \multicolumn{4}{c}{ Matched Control } & \multicolumn{2}{c}{ Unmatched Control } \\
\hline Ethnicity & Female & Male & Total & Female & Male & Total & Female & Male & Total \\
\hline NZ European & 10 & 6 & 16 & 10 & 7 & 17 & 12 & 32 & 44 \\
Māori & 8 & 2 & 10 & 3 & 1 & 4 & 3 & 2 & 5 \\
Pacific Islander & 5 & 2 & 7 & 2 & 3 & 5 & 4 & 4 & 8 \\
Asian & 1 & 1 & 2 & 0 & 1 & 1 & 0 & 5 & 5 \\
Other & 1 & 2 & 3 & 1 & 1 & 2 & 1 & 6 & 7 \\
\hline Totals & 25 & 13 & 38 & 16 & 13 & 29 & 20 & 49 & 69 \\
\hline
\end{tabular}




\section{Differences between programme and control groups after programme}

\section{Differences between Kiwi ACE and its Matched Control Group}

Repeated measures MANOVA were run to compare Kiwi ACE and its matched control group scores for each scale from T1-T4 and follow up MANOVA analyses were run to determine at what time points the significant differences occurred.

\section{Prediction 1: Kiwi ACE would decrease Depressive Symptoms}

.As predicted, the results showed that there was a significant interaction for group and depression scores, $F(1,50)=5.25, p=.03$, partial $\eta 2=.10$. Follow up analyses revealed that the Kiwi ACE's depression scores were not significantly different at T1 to the control group $(\mathrm{T} 1: F(1,50)=.001, p=.98$, partial $\eta 2=.001)$, but were significantly lower than the control group at all time points after the programme was run $(\mathrm{T} 2: F(1,50)=3.95, p=.05$, partial $\eta 2=.07 ; \mathrm{T} 3: F(1,50)=5.10, p=.03$, partial $\eta 2=.09 ; \mathrm{T} 4: F(1,50)=4.50, p=.04$, partial $\eta 2=.08$ ). This set of results indicates that Kiwi ACE was successful in lowering depression scores for up to one year after students participated in the programme.

\section{Prediction 2: Kiwi ACE would Decrease Maladaptive Coping}

As predicted, there was a significant interaction for group and maladaptive coping, $F(1,50)=17.87, p=.001$, partial $\eta 2=.26$. Contrasts revealed that Kiwi ACE's maladaptive coping scores were not significantly different at $\mathrm{T} 1$ to the control group $(\mathrm{T} 1: F(1,50)=.12$, $p=.74$, partial $\eta 2=.001$ ) and were significantly lower than the control group at all time points after the programme was run $(\mathrm{T} 2: F(1,50)=13.90, p=.001$, partial $\eta 2=.22 ; \mathrm{T} 3: F(1$, $50)=17.98, p=.00$, partial $\eta 2=.26 ; \mathrm{T} 4: F(1,50)=8.94, p=.001$, partial $\eta 2=.15)$. This set of results indicates that Kiwi ACE was successful in lowering maladaptive scores for up to one year after students participated in the programme.

\section{Prediction 3: Kiwi ACE would increase Adaptive Coping}

It was predicted that Kiwi ACE would also increase adaptive coping, however, the results did not support this prediction: there was no significant difference at any time point between Kiwi ACE and the control group for adaptive coping. 


\section{Prediction 4: Kiwi ACE would decrease Total Difficulties}

It was predicted that Kiwi ACE would decrease participants reports of total difficulties, however, only weak effects were obtained for difficulties, in that all results were marginally significant.

\section{Prediction 5: Kiwi ACE would increase Resilience}

It was predicted that Kiwi ACE would increase resilience, however, resilience obtained inconsistent effects, in that it was only significant at T3, see table 11.

The following results were obtained for the remaining outcomes that were not predicted:

Well-being. There was a significant interaction for group and well-being, $F(1,50)=$ $11.44, p=.001$, partial $\eta 2=.19$. Contrasts revealed that Kiwi ACE's well-being scores were not significantly different at $\mathrm{T} 1$ to the control group $(\mathrm{T} 1: F(1,50)=.45, p=.51$, partial $\eta 2=.01)$ and significantly higher than the control group at all time points after the programme was run $(\mathrm{T} 2: F(1,50)=9.78, p=.001$, partial $\eta 2=.16$; T3: $F(1,50)=12.52, p=$ .00 , partial $\eta 2=.20 ; \mathrm{T} 4: F(1,50)=8.94, p=.001$, partial $\eta 2=.15)$. This set of results indicates that Kiwi ACE was successful in increasing well-being scores for up to one year after students participated in the programme.

There were inconsistent effects for, happiness, gratitude, and life satisfaction were obtained in that they were only significant at T3, see table 11 .

To summarise, as predicted Kiwi ACE decreased depressive symptoms and maladaptive coping for up to one year after the programme ended. Kiwi ACE also increased wellbeing, however, only weak effects were found for all other variables, with the exception of adaptive coping, which showed no significant change over time. 
Table 11. Weak effects in Resilience, Happiness, Life Satisfaction and Difficulties when comparing Kiwi ACE with matched control

\begin{tabular}{|c|c|c|c|c|c|c|c|c|c|c|c|c|c|c|c|}
\hline \multirow[t]{2}{*}{ Variable } & \multicolumn{3}{|c|}{ Main effect } & \multicolumn{3}{|c|}{$\mathrm{T} 1$} & \multicolumn{3}{|c|}{$\mathrm{T} 2$} & \multicolumn{3}{|c|}{ T3 } & \multicolumn{3}{|c|}{$\mathrm{T} 4$} \\
\hline & $F$ & $p$ & $\mathrm{~h}$ & $F$ & $p$ & $\mathrm{~h}$ & $F$ & $p$ & $\mathrm{~h}$ & $F$ & $p$ & $\mathrm{~h}$ & $F$ & $p$ & $\mathrm{~h}$ \\
\hline difficulties & 3.01 & 0.09 & 0.06 & 0.02 & 0.88 & 0.00 & 3.47 & 0.07 & 0.07 & 3.83 & 0.06 & 0.07 & 2.96 & 0.09 & 0.06 \\
\hline resilience & 3.03 & 0.09 & 0.06 & 0.03 & 0.87 & 0.00 & 1.29 & 0.26 & 0.03 & 8.07 & 0.01 & 0.14 & 2.53 & 0.12 & 0.05 \\
\hline happiness & 1.48 & 0.23 & 0.03 & 0.14 & 0.71 & 0.00 & 2.08 & 0.16 & 0.04 & 3.96 & 0.05 & 0.07 & 0.00 & 0.99 & 0.00 \\
\hline gratitude & 2.93 & 0.09 & 0.06 & 0.02 & 0.89 & 0.00 & 2.02 & 0.16 & 0.04 & 8.50 & 0.01 & 0.15 & 1.16 & 0.29 & 0.02 \\
\hline life satisfaction & 1.34 & 0.25 & 0.03 & 0.02 & 0.90 & 0.00 & 0.05 & 0.83 & 0.00 & 6.40 & 0.02 & 0.11 & 1.02 & 0.32 & 0.02 \\
\hline
\end{tabular}




\section{Differences between PAL and its matched control group}

Repeated measures MANOVA were run to compare PAL and its matched control group scores for each scale from T1-T4 and follow up MANOVA analyses were run to see at what time points the significant differences were.

\section{Prediction 8: PAL would increase Psychological Well-being}

As predicted, there was a significant interaction for group and well-being, $F(1,66)=$ $6.08, p=.02$, partial $\eta 2=.08$. Contrasts revealed that PAL's well-being scores were not significantly different at $\mathrm{T} 1$ to the control group $(\mathrm{T} 1: F(1,66)=.09, p=.77$, partial $\eta 2=.001$ but were significantly higher than the control group at all time points after the programme was run $(\mathrm{T} 2: F(1,66)=6.29, p=.02$, partial $\eta 2=.10$; $\mathrm{T} 3: F(1,66)=3.47, p=$ .067 , partial $\eta 2=.06 ; \mathrm{T} 4: F(1,66)=5.92, p=.02$, partial $\eta 2=.09)$. These set of results indicate that PAL was successful in increasing well-being scores for up to one year after students participated in the programme.

\section{Prediction 9: PAL would increase Satisfaction with Life}

As predicted, there was a significant interaction for group and satisfaction with life, $\mathrm{F}(1,66)=4.41, p=.04$, partial $\eta 2=.06$. Contrasts revealed that PAL's satisfaction with life scores were not significantly different at $\mathrm{T} 1$ to the control group $(\mathrm{T} 1: F(1,66)=.001, p=$ .99 , partial $\eta 2=.001$ ) but significantly higher than the control group at all time points after the programme was run $(\mathrm{T} 2: F(1,66)=5.24, p=.03$, partial $\eta 2=.07$; T3: $F(1,66)=5.75, p$ $=.02$, partial $\eta 2=.08 ; \mathrm{T} 4: F(1,66)=5.20, p=.03$, partial $\eta 2=.07)$. These sets of results indicate that PAL was successful in increasing satisfaction with life scores for up to one year after students participated in the programme.

\section{Prediction 10: PAL would increase Subjective Happiness}

As predicted, there was a significant interaction for group and happiness, $F(1,66)=$ $3.79, p=.056$, partial $\eta 2=.05$. Contrasts revealed that PAL's happiness scores were not significantly different at $\mathrm{T} 1$ to the control group $(\mathrm{T} 1: F(1,66)=.03, p=.88$, partial $\eta 2=.001$ ) but significantly higher than the control group at all time points after the programme was run $(\mathrm{T} 2: F(1,66)=3.74, p=.058$, partial $\eta 2=.05 ; \mathrm{T} 3: F(1,66)=6.37, p=$ .01 , partial $\eta 2=.09 ; \mathrm{T} 4: F(1,66)=4.71, p=.03$, partial $\eta 2=.07)$. These set of results indicate that PAL was successful in increasing happiness scores for up to one year after students participated in the programme. 


\section{Prediction 11: PAL would increase Gratitude}

As predicted, there was a significant interaction for group and gratitude, $\mathrm{F}(1,66)=$ 5.95, $p=.02$, partial $\eta 2=.08$. Contrasts revealed that PAL's gratitude scores were not significantly different at $\mathrm{T} 1$ to the control group $(\mathrm{T} 1: F(1,66)=.001, p=.95$, partial $\eta 2=.00$ ) but significantly higher than the control group at all time points after the programme was run $(\mathrm{T} 2: F(1,66)=7.13, p=.01$, partial $\eta 2=.10 ; \mathrm{T} 3: F(1,66)=7.56, p=$ .01 , partial $\eta 2=.10 ; \mathrm{T} 4: F(1,66)=6.61, p=.01$, partial $\eta 2=.09)$. These set of results indicate that PAL was successful in increasing gratitude scores for up to one year after students participated in the programme.

\section{Prediction 12: PAL would increase Personal Resilience}

As predicted, there was a significant interaction for group and resilience, $F(1,66)=$ $6.48, p=.01$, partial $\eta 2=.09$. Contrasts revealed that PAL's resilience scores were not significantly different at $\mathrm{T} 1$ to the control group $(\mathrm{T} 1: F(1,66)=.99, p=.32$, partial $\eta 2=.01$ ) but significantly higher than the control group at all time points after the programme was run $(\mathrm{T} 2: F(1,66)=3.94, p=.05$, partial $\eta 2=.05 ; \mathrm{T} 3: F(1,66)=6.85, p=$ .01 , partial $\eta 2=.10 ; \mathrm{T} 4: F(1,66)=6.83, p=.01$, partial $\eta 2=.09)$. Indicating that PAL was successful in increasing resilience scores until one year after students participated in the programme.

\section{Prediction 13: PAL would decrease Depressive Symptoms}

As predicted, there was a significant interaction for group and depression scores, $F(1$, $66)=9.85, p=.001$, partial $\eta 2=.13$. Follow up analyses revealed that the PAL's depression scores were not significantly different at $\mathrm{T} 1$ to the control group $(\mathrm{T} 1: F(1,66)=.08, p=.78$, partial $\eta 2=.001$ ) but were significantly lower than the control group at all time points after the programme was run $(\mathrm{T} 2: F(1,66)=3.62, p=.06$, partial $\eta 2=.05 ; \mathrm{T} 3: F(1,66)=10.17$, $p=.00$, partial $\eta 2=.14 ; \mathrm{T} 4: F(1,66)=14.40, p=.001$, partial $\eta 2=.18)$. These set of results indicate that PAL was successful in lowering depression scores for up to one year after students participated in the programme.

The following results were obtained for the outcomes not predicted:

Difficulties. The results showed that the there was a significant interaction for group and difficulties experienced, $F(1,66)=7.33, p=.01$, partial $\eta 2=.10$. Follow up analyses 
revealed that PAL's total difficulties scores were not significantly different at T1 to the control group $(\mathrm{T} 1: F(1,66)=.00, p=.99$, partial $\eta 2=.001)$ and were significantly lower than the control group at all time points after the programme was run $(\mathrm{T} 2: F(1,66)=3.79, p$ $=.06$, partial $\eta 2=.06 ; \mathrm{T} 3: F(1,66)=9.45, p=.00$, partial $\eta 2=.12 ; \mathrm{T} 4: F(1,66)=13.40, p$ $=.00$, partial $\eta 2=.16$ ). These results indicate that PAL was successful in lowering student's reported experience of difficulties until one year after students participated in the programme.

There was no significant difference between PAL and the control group for adaptive or maladaptive coping at any time points.

To summarise, PAL was effective at increasing all variables predicted, and also decreased depression and difficulties for up to one year after students participated in the programme. Whereas maladaptive and adaptive coping showed no change in the follow-up tests.

\section{Differences Between the Kiwi ACE and PAL Programmes}

A MANOVA was run to determine if there were any significant differences on the eight scales over time between the programmes. There were no significant interactions between the groups on any scale at any time points.

\section{Significance Criterion Adjusted}

Investigating how the programme had an effect resulted in a large number of mediational analyses (approximately 35), because such a large number of analyses was conducted, the significance criterion used was adjusted from $p \leq .05$ to $p \leq .01$. This is less conservative than a Bonferroni adjustment, which would have resulted in a criterion of $p \leq$ .001 . However, because the tests were not perfectly independent, as the Bonferroni adjustment assumes, it seemed reasonable to use this intermediate value.

\section{The Mediators of Kiwi ACE}

\section{Prediction 6:}

Total difficulties would function as a mediator between Kiwi ACE and Depression

It was predicted that total difficulties experienced at T2 and T3 would mediate the relationship from group (the difference between Kiwi ACE and the control group) to depression scores at $\mathrm{T} 4$, in other words, the programme was expected to lead to a decrease in difficulties, which in turn was expected to lead to lower depression. However mediational 
analysis did not support this prediction, and revealed that difficulties did not significantly mediate this relationship.

\section{Prediction 7:}

Maladaptive coping would function as a mediator between Kiwi ACE and Depression

It was predicted that maladaptive coping at $\mathrm{T} 2$ and $\mathrm{T} 3$ would mediate the relationship from group to depression scores at $\mathrm{T} 4$, in other words the programme was expected to lead to a decrease in maladaptive coping which in turn was expected to lead to lower depression. However mediational analyses revealed that maladaptive coping did not significantly mediate this relationship.

\section{Exploratory Mediation}

Exploratory mediation analyses were conducted to investigate whether there were mediational relationships that would help to understand the mechanisms by which Kiwi ACE caused the decrease in depression and increase in well-being. The subscales of well-being, total difficulties and depression were used in these mediational analyses to further explore these relationships.

Environmental Mastery, a subscale of well-being, was used as a mediator to explore the relationship from group to depression. The analysis revealed that Environmental Mastery (at T2 and T3) mediated the relationship between group and T4 depression (see Table 12), indicating that one of the ways in which Kiwi ACE helped to decrease depression was by increasing students' mastery.

Total difficulties did not significantly mediate the relationship between group and well-being, but to investigate this further the subscales of total difficulties were used in mediational analyses. The results revealed that emotional difficulties at T2 and T3 mediated the relationship between group and well-being at T4 (see Table 12). Indicating that one of the ways that Kiwi ACE helped to increase well-being was by decreasing students' emotional difficulties.

The subscales of the depression scale were also used in mediational analyses. Anhedonia (at T2 and T3) was found to mediate the relationship from group effect to T4 well-being (see table 12). This mediation indicates that Kiwi ACE helped to increase wellbeing by decreasing Anhedonia. It is important to note that none of the other depression subscales mediated this relationship - including Negative Mood.

To summarise, the results from the mediation analyses indicate that Kiwi ACE helped to decrease students' depressive scores by increasing students' sense of environmental 
mastery and helped to increase students' well-being scores by decreasing students' experience of emotional difficulties and by lowering anhedonia (lack of pleasure). 
Table 12. Standardised indirect effects from mediational relationships between variables in Kiwi ACE

\begin{tabular}{|c|c|c|c|c|c|c|}
\hline & & \multicolumn{5}{|c|}{ Standardised Indirect effects } \\
\hline & & Group-T2-T3 & Group-T2-T3-T4 & Group-T2-T4 & Group-T3-T4 & T2-T3-T4 \\
\hline Mediator: & Size & 0.13 & -0.22 & -0.10 & -0.21 & -0.24 \\
\hline Mastery & SE & 0.07 & 0.08 & 0.06 & 0.08 & 0.08 \\
\hline Outcome: & $\mathrm{p}$ & 0.03 & 0.01 & 0.02 & 0.00 & 0.00 \\
\hline \multirow[t]{2}{*}{ Depression } & $\mathrm{CI}$ & .04 to .25 & -.38 to -.10 & -.23 to -.02 & -.37 to -.09 & -.39 to -.12 \\
\hline & & Group-T2-T3 & Group-T2-T3-T4 & Group-T2-T4 & Group-T3-T4 & T2-T3-T4 \\
\hline Mediator: & Size & -0.14 & 0.17 & 0.05 & 0.17 & -.33 \\
\hline Emotional D & SE & 0.08 & 0.08 & 0.05 & 0.08 & 0.10 \\
\hline Outcome: & $p$ & 0.09 & 0.02 & 0.09 & 0.01 & 0.00 \\
\hline \multirow[t]{2}{*}{ Well-being } & CI & -.28 to -.01 & .05 to .30 & .00 to .18 & .07 to .31 & -.51 to -.18 \\
\hline & & Group-T2-T3 & Group-T2-T3-T4 & Group-T2-T4 & Group-T3-T4 & T2-T3-T4 \\
\hline Mediator: & Size & -0.08 & 0.15 & 0.06 & 0.13 & -0.07 \\
\hline Anhedonia & SE & 0.05 & 0.07 & 0.05 & 0.07 & 0.05 \\
\hline Outcome: & $p$ & 0.07 & 0.01 & 0.07 & 0.02 & 0.07 \\
\hline Well-being & $\mathrm{CI}$ & -.19 to -.01 & .04 to .30 & .01 to .19 & .04 to .27 & -.19 to -.01 \\
\hline
\end{tabular}




\section{The Mediators of PAL}

Prediction 14: Happiness would function as a mediator between PAL and Well-being

It was predicted that the relationship between group (PAL vs control) and well-being would be mediated by happiness. In other words that PAL was expected to lead to an increase in happiness which in turn was expected to lead to an increase in well-being. Figure 1 depicts happiness experienced at $\mathrm{T} 2$ mediating the relationship from group effect to well-being at T4, and as predicted the standardised indirect effect from group, through T2 happiness to T4 well-being was significant (see Figure 1). Figure 2 depicts happiness experienced at T3 mediating the relationship from group effect to well-being at $\mathrm{T} 4$, and as predicted the standardised indirect effect from group, through T2 happiness to T4 well-being was significant (see Figure 2). Figure 3 depicts happiness experienced at T2 and T3 mediating the relationship from group effect to well-being at T4. The standardised direct effect between group and well-being at T4 decreased substantially when controlling for happiness at T2 and T3. As predicted the standardised indirect effect from group, through T2 and T3 happiness to T4 well-being was significant (see Figure 3). This mediation indicates that one of the ways PAL increased well-being was by increasing students' happiness.

\section{Prediction 15: Gratitude would function as a mediator between PAL and Well-being.}

It was predicted that gratitude at T2 and T3 would mediate the relationship from group (the difference between PAL and the control group) to well-being scores at T4. In other words that PAL was expected to lead to an increase in gratitude which in turn was expected to lead to an increase in well-being. Figure 4 depicts gratitude experienced at T2 mediating the relationship from group effect to well-being at $\mathrm{T} 4$, and as predicted the standardised indirect effect from group through T2 gratitude to T4 well-being proved to be statistically significant (see Figure 4). Figure 5 depicts gratitude experienced at T3 mediating the relationship from group effect to well-being at $\mathrm{T} 4$, and as predicted the standardised indirect effect from group, through T2 gratitude to T4 well-being was significant (see Figure 5). Figure 6 depicts gratitude experienced at T2 and T3 mediating the relationship from group effect to well-being at T4. The standardised direct effect between group and well-being at T4 decreased substantially when controlling for gratitude at T2 and T3. As predicted the standardised indirect effect from group, through T2 and T3 gratitude to T4 was significant (see Figure 6). This mediation indicates that one of the ways PAL increased well-being was by increasing the student's gratitude. 


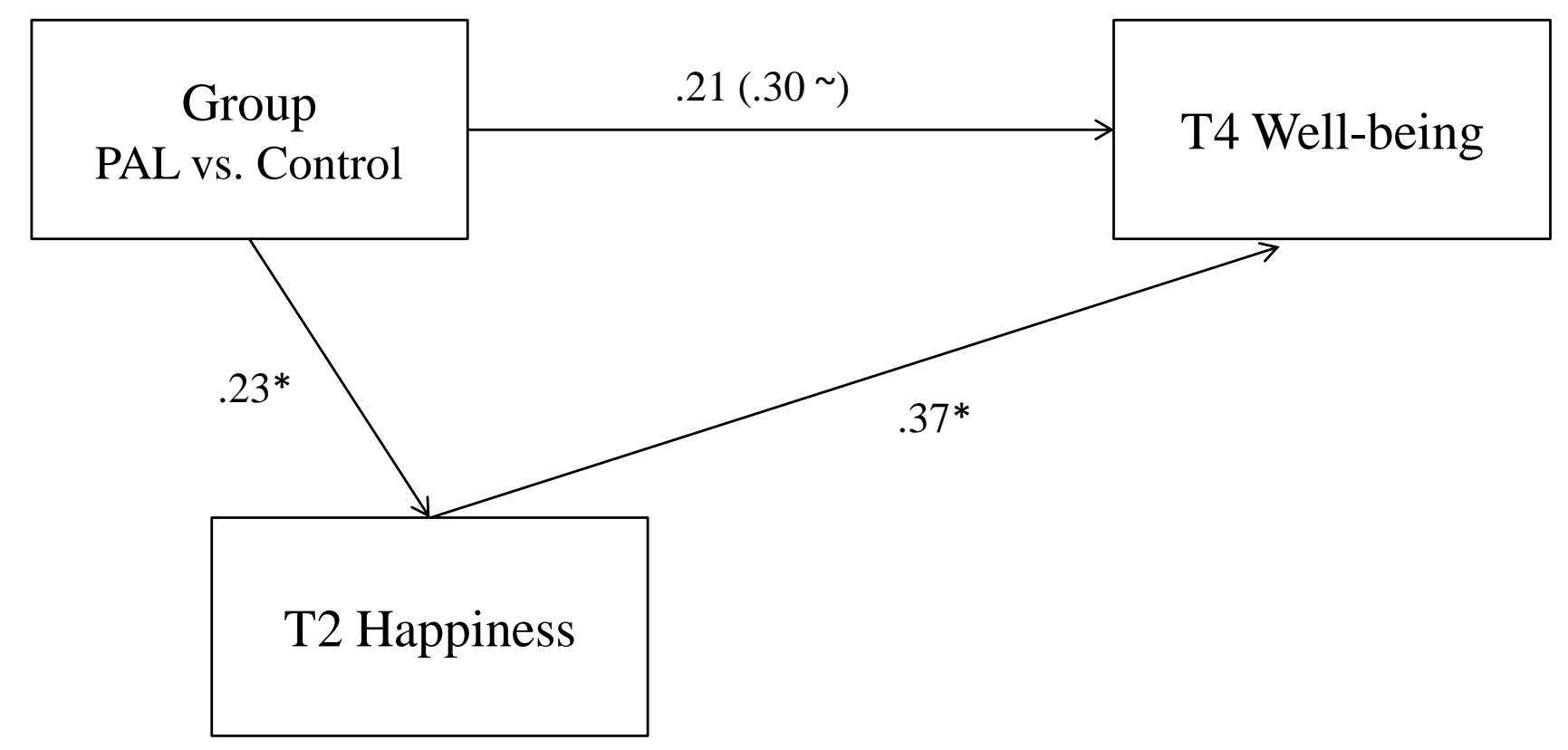

Figure 1. Standardised coefficients of the total, direct, and indirect effects of group on Well-being mediated by T2 Happiness. (Estimate of total effect is presented in parenthesis).

Indirect effect: Group to T2 Happiness to T4 Well-being, $\mathrm{Size}=.09, \mathrm{SE}=.05, p=.02, \mathrm{CI}=.02$ to .19

$* * p<.001 * p<.01 \sim p=.05$ 


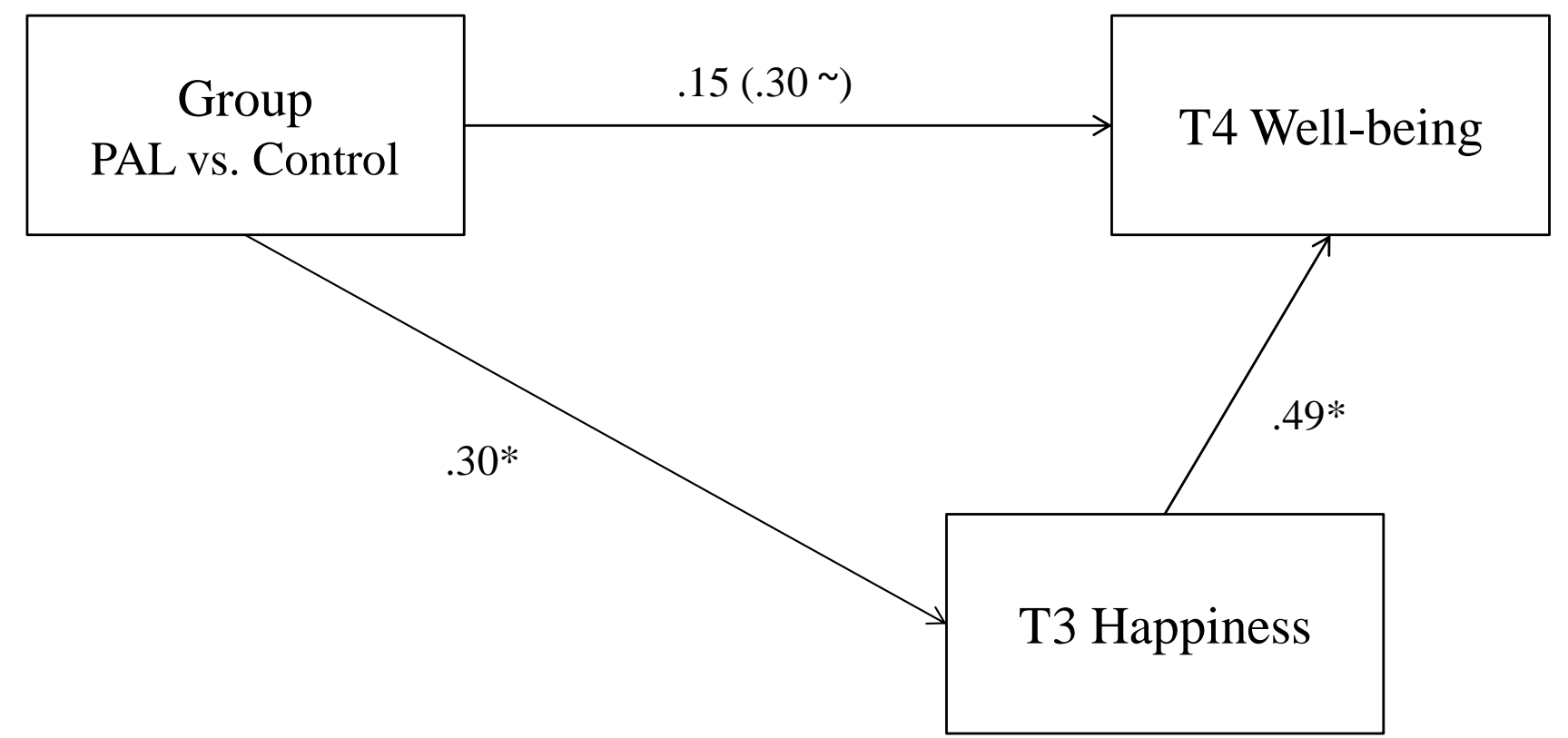

Figure 2. Standardised coefficients of the total, direct, and indirect effects of group on Well-being mediated by T3 Happiness. (Estimate of total effect is presented in parenthesis).

Standardised indirect effect:

Group to T3 Happiness to T4 Well-being, Size $=.15, \mathrm{SE}=.06, p=.01, \mathrm{CI}=.06$ to .27

$* * p<.001 * p<.01 \sim p=.05$ 


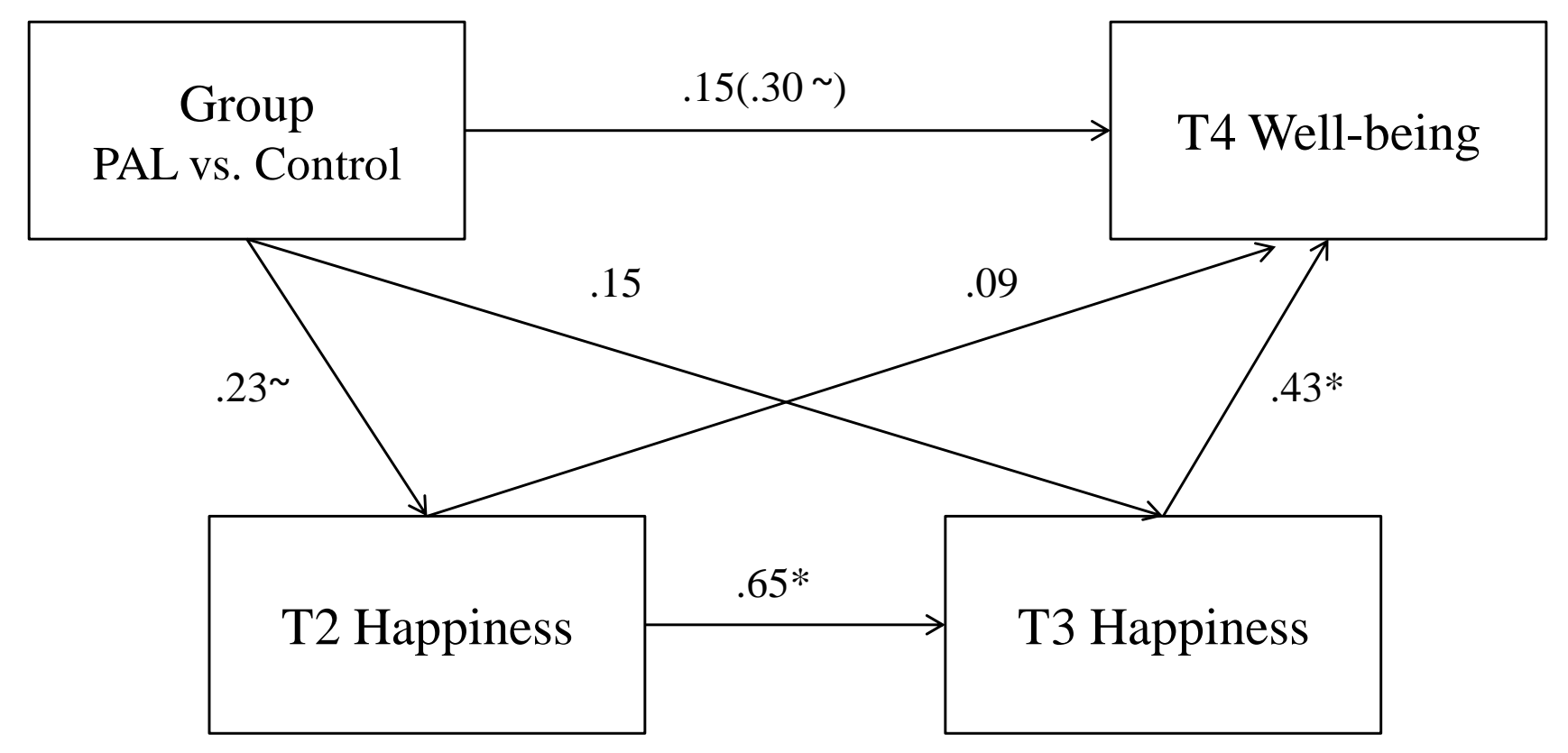

Figure 3. Standardised coefficients of the total, direct, and indirect effects of group on Well-being mediated by T2 \& T3 Happiness. (Estimate of total effect is presented in parenthesis).

Standardised indirect effect:

Group to T2 Happiness to T3 Happiness to T4 Well-being, Size $=.14, \mathrm{SE}=.06, p=.01, \mathrm{CI}=.05$ to .27

$* * p<.001 * p<.01 \sim p=.05$ 


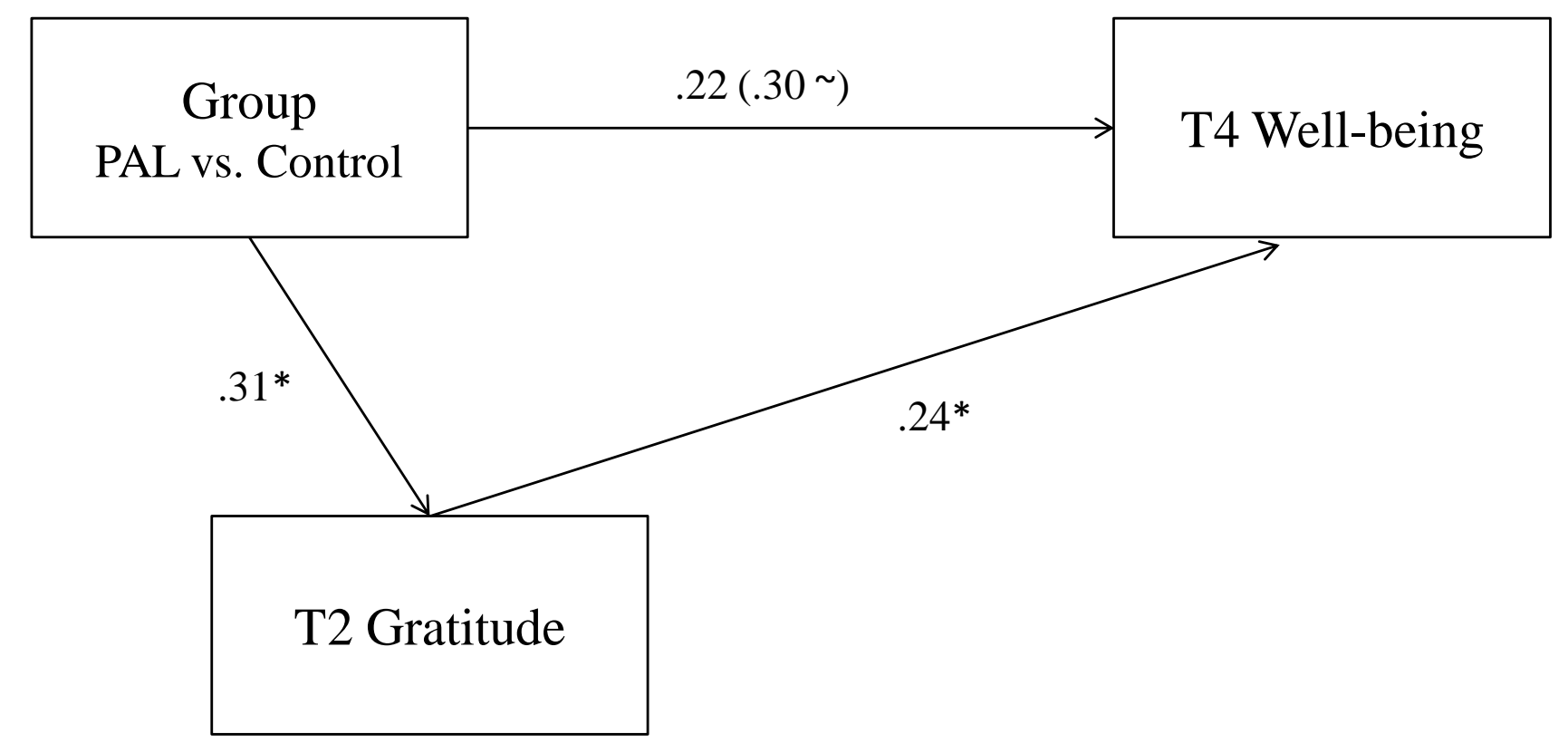

Figure 4. Standardised coefficients of the total, direct, and indirect effects of group on Well-being mediated by T2 Gratitude. (Estimate of total effect is presented in parenthesis).

Indirect effect: Group to T2 Gratitude to T4 Well-being, Size $=.08, \mathrm{SE}=.05, p=.02, \mathrm{CI}=.02$ to .18

$* * p<.001 * p<.01 \sim p=.05$ 


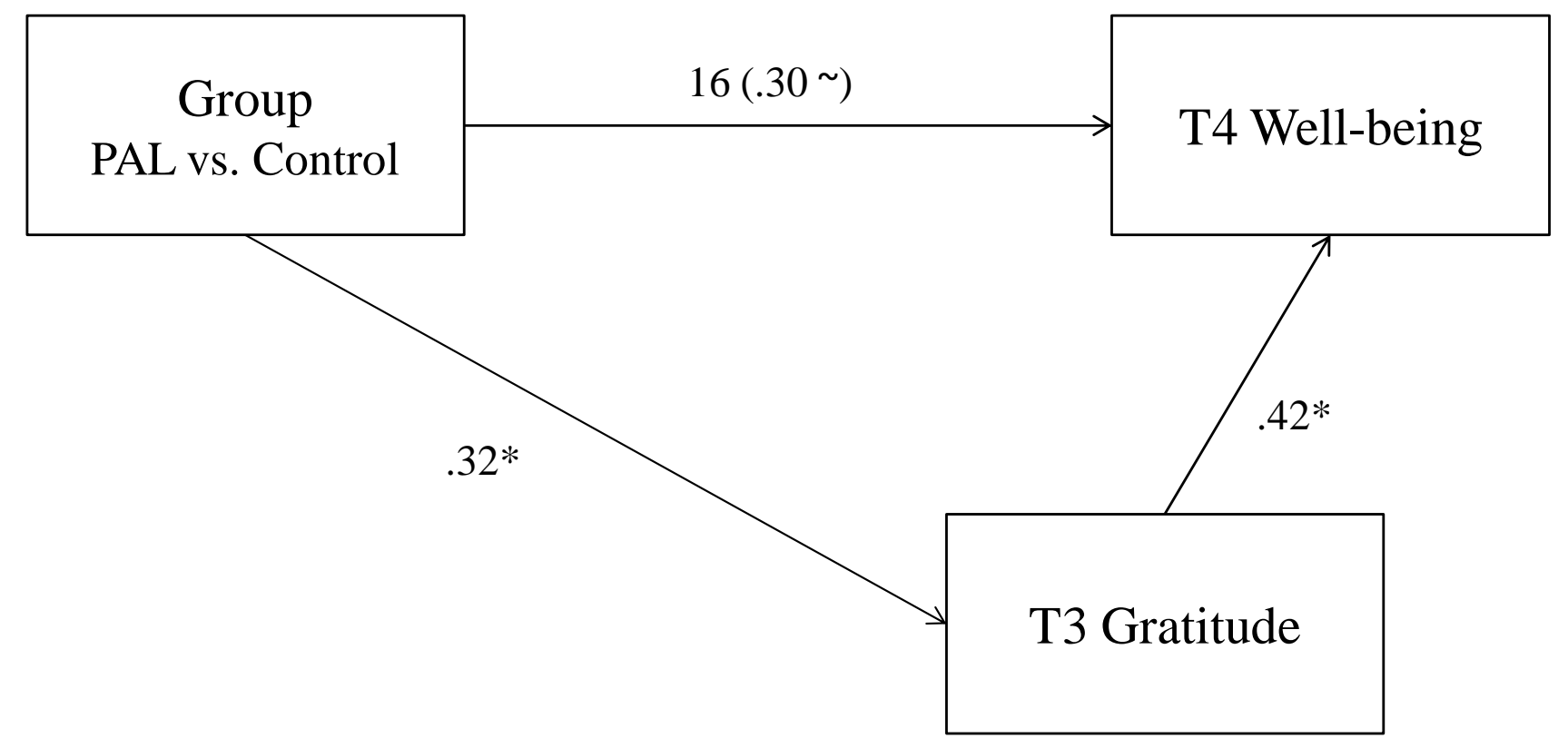

Figure 5. Standardised coefficients of the total, direct, and indirect effects of group on Well-being mediated by T3 Gratitude. (Estimate of total effect is presented in parenthesis).

Standardised indirect effect:

Group to T3 Gratitude to T4 Well-being, Size $=-.14, \mathrm{SE}=.06, p=.00, \mathrm{CI}=.06$ to .25

$* * p<.001 * p<.01 \sim p=.05$ 


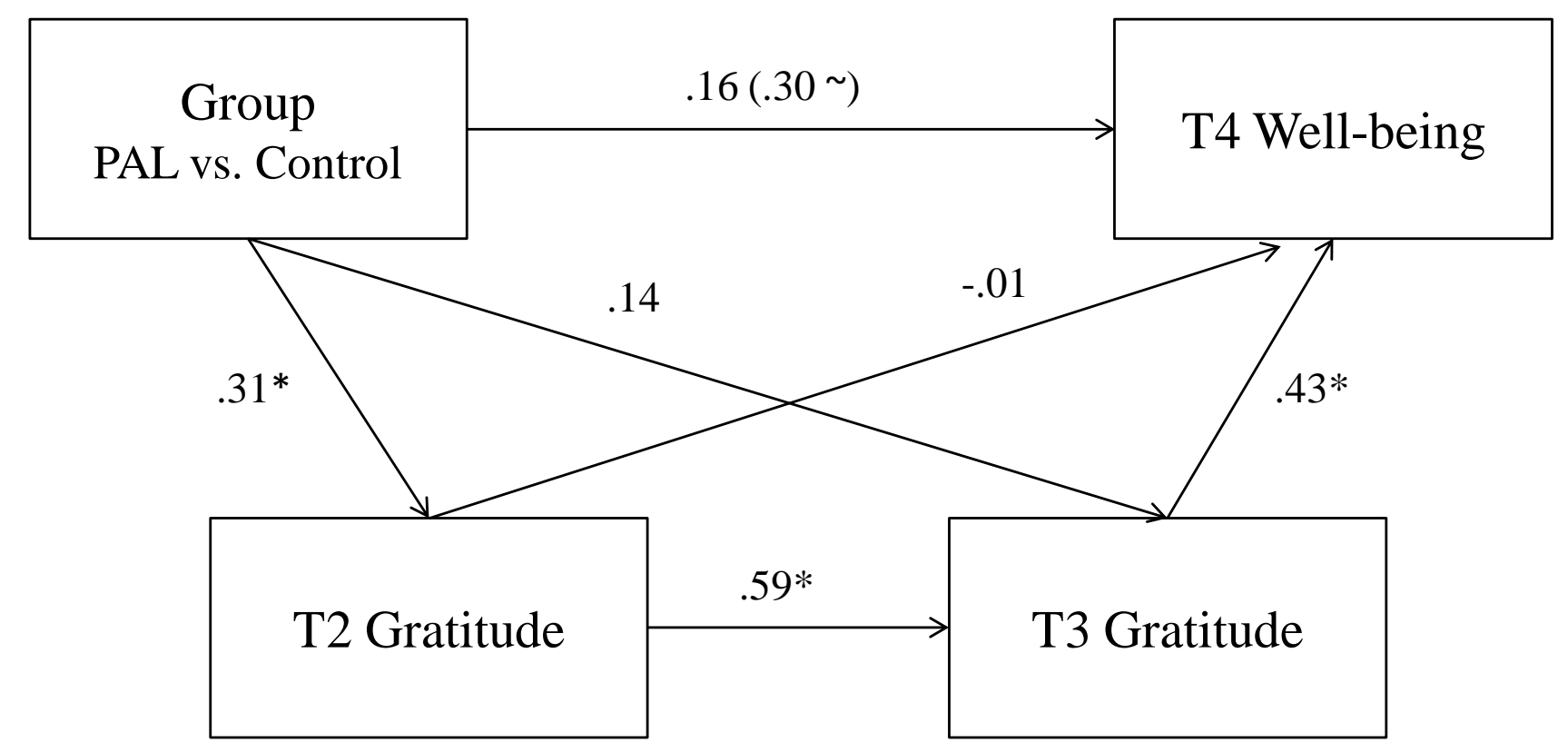

Figure 6. Standardised coefficients of the total, direct, and indirect effects of group on Well-being mediated by T2 \& T3 Gratitude. (Estimate of total effect is presented in parenthesis).

Standardised indirect effect:

Group to T2 Gratitude to T3 Gratitude to T4 Well-being, Size $=.13, \mathrm{SE}=.06, p=.01, \mathrm{CI}=.04$ to .25

$* * p<.001 * p<.01 \sim p=.05$ 


\section{Exploratory Mediation}

Exploratory mediation analyses were conducted to investigate whether there were mediational relationships that would help to further understand how PAL caused the decrease in depression and the increase in well-being and resilience.

To explore the relationship between group effect and resilience, mediational analyses were conducted using gratitude at T2 and T3 as the mediators. Table 12 shows that gratitude mediated the relationship from group (PAL vs. control) to resilience at T4, as all standardised indirect effects were significant. Table 12 also shows that satisfaction with life mediated the relationship from group effect to T4 resilience, and happiness marginally mediated the relationship between group effect and $\mathrm{T} 4$ resilience. These mediations indicate that some of the ways in which PAL helped to increase resilience was by increasing students' gratitude, satisfaction with life and happiness.

To further explore the relationship between group, resilience and well-being, a mediational analysis was conducted using the subscales of resilience. The analyses revealed that meaningfulness at T2 and T3 mediated the relationship from group to well-being at T4, indicating that another way PAL helped to increase well-being was by increasing the meaning in students' lives (Table 12 shows that all standardised indirect effects were significant).

Further meditational analysis was conducted to investigate if gratitude would also meditate the relationship from PAL to happiness. The analyses revealed that gratitude at T2 and T3 mediated the relationship from group to happiness at T4, indicating that gratitude also helped to increase happiness (Table 12 shows that all standardised indirect effects were significant).

To explore the relationship between group effect, well-being and depression, a mediational analysis was conducted using the subscales of well-being and depression. The mediational analyses showed that anhedonia at T2 and T3 mediated the relationship between group effect and well-being at T4 (Table 12 shows that all standardised indirect effects were significant). These mediations indicate that that PAL helped to increase well-being by lowering anhedonia. Table 12 also shows that meaningfulness at T2 and T3 mediated the relationship between group effect and depression at T4, gratitude at T2 and T3 mediated the relationship between group effect and depression at $\mathrm{T} 4$, and satisfaction with life at $\mathrm{T} 2$ and $\mathrm{T} 3$ mediated the relationship between group effect and depression at T4. These mediations indicate that by increasing meaningfulness, gratitude and satisfaction with life PAL helped to decrease depression. 
To summarise, some of the ways in which PAL helped to increase well-being scores was by increasing gratitude, happiness and meaning, and by lowering anhedonia. Some of the ways in which PAL helped to increase resilience scores was through increasing happiness, gratitude and satisfaction with life. Some of the ways in which PAL helped to decrease depressive scores was by increasing meaning, gratitude and satisfaction with life. 
Table 13. Standardised indirect effects from mediational relationships between variables in PAL

\begin{tabular}{|c|c|c|c|c|c|c|}
\hline \multicolumn{7}{|c|}{ Standardised indirect effects } \\
\hline & & Group-T2-T3 & Group-T2-T3-T4 & Group-T2-T4 & Group-T3-T4 & T2-T3-T4 \\
\hline Mediator: & Size & 0.19 & 0.13 & 0.08 & 0.14 & 0.25 \\
\hline Gratitude & SE & 0.07 & 0.06 & 0.05 & 0.06 & 0.09 \\
\hline Outcome: & $p$ & 0.00 & 0.01 & 0.02 & 0.00 & 0.00 \\
\hline \multirow[t]{2}{*}{ Well-Being } & CI & .07 to .30 & .04 to .25 & .02 to .18 & .06 to .25 & .11 to .41 \\
\hline & & Group-T2-T3 & Group-T2-T3-T4 & Group-T2-T4 & Group-T3-T4 & T2-T3-T4 \\
\hline Mediator: & Size & 0.15 & 0.14 & 0.09 & 0.15 & 0.28 \\
\hline Happiness & SE & 0.07 & 0.06 & 0.05 & 0.06 & 0.10 \\
\hline Outcome: & $p$ & 0.06 & 0.01 & 0.02 & 0.01 & 0.00 \\
\hline \multirow[t]{2}{*}{ Well-being } & CI & .01 to .26 & .05 to .27 & .02 to .19 & .06 to .27 & .13 to .46 \\
\hline & & Group-T2-T3 & Group-T2-T3-T4 & Group-T2-T4 & Group-T3-T4 & T2-T3-T4 \\
\hline Mediator: & Size & 0.19 & 0.18 & 0.12 & 0.22 & 0.28 \\
\hline Gratitude & SE & 0.07 & 0.07 & 0.05 & 0.08 & 0.08 \\
\hline Outcome: & $p$ & 0.00 & 0.00 & 0.00 & 0.01 & 0.00 \\
\hline \multirow[t]{2}{*}{ Resilience } & $\mathrm{CI}$ & .07 to .27 & .08 to .31 & .05 to .23 & .10 to .36 & .15 to .43 \\
\hline & & Group-T2-T3 & Group-T2-T3-T4 & Group-T2-T4 & Group-T3-T4 & T2-T3-T4 \\
\hline Mediator: & Size & 0.19 & 0.14 & 0.10 & 0.13 & 0.30 \\
\hline Satisfaction & SE & 0.08 & 0.06 & 0.05 & 0.06 & 0.11 \\
\hline Outcome: & $p$ & 0.02 & 0.01 & 0.01 & 0.01 & 0.00 \\
\hline \multirow[t]{2}{*}{ Resilience } & $\mathrm{CI}$ & .04 to .30 & .05 to .26 & .03 to .20 & .05 to .25 & .14 to .49 \\
\hline & & Group-T2-T3 & Group-T2-T3-T4 & Group-T2-T4 & Group-T3-T4 & T2-T3-T4 \\
\hline Mediator: & Size & 0.15 & 0.11 & 0.09 & 0.10 & 0.07 \\
\hline Happiness & SE & 0.07 & 0.06 & 0.05 & 0.05 & 0.10 \\
\hline Outcome: & $p$ & 0.06 & 0.03 & 0.02 & 0.01 & 0.44 \\
\hline \multirow[t]{2}{*}{ Resilience } & CI & .01 to .26 & .02 to .22 & .02 to .20 & .03 to .21 & -.10 to .24 \\
\hline & & Group-T2-T3 & Group-T2-T3-T4 & Group-T2-T4 & Group-T3-T4 & T2-T3-T4 \\
\hline Mediator: & Size & 0.15 & 0.12 & 0.09 & 0.10 & 0.21 \\
\hline Meaning & SE & 0.07 & 0.06 & 0.05 & 0.06 & 0.08 \\
\hline Outcome: & $p$ & 0.01 & 0.03 & 0.01 & 0.02 & 0.00 \\
\hline Well-being & $\mathrm{CI}$ & .05 to .26 & .03 to .23 & .03 to .19 & .03 to .21 & .10 to .35 \\
\hline
\end{tabular}




\title{
Qualitative Results
}

Qualitative research was used to gain an understanding of how both programmes were received and experienced by the students and the leaders. The following qualitative results are based on the themes that were identified from the discussions held and the feedback forms completed by students and leaders at the end of the programme. (Please see method section, p.98 for more details on the procedure and feedback forms). The forms were coded and percentages were calculated for each question. The discussions that took place, and the leaders' checklists and comments, were used to expand answers further and used in the coding and synthesis of the qualitative results. When looking at the percentages it is important to take into account that some open-ended questions were not answered by every participant. Additionally some students gave more than one answer to a question.

The topics explored in the qualitative results below are: students' motivation to join the programme, a look into the possible stigma experienced because of the programme, group dynamics, how process and content of sessions were received by students and leaders, concerns about the programme, and suggested improvements.

\section{Kiwi ACE Qualitative Results}

\section{Why Students Joined the Group}

Students gave one or more of the following reasons to the question of why they agreed to participate in Kiwi ACE when they were initially asked to join: $45 \%$ of the Kiwi ACE participants felt that the group might help them in some way and that they would learn from it, examples of these responses include:

\author{
Because I thought it could help me with some problems \\ Because I thought that I would help me at school and wherever I may be. \\ It is good think to join and I can learn lots of things from this
}

Fourteen percent of students wanted to participate so that they could skip class, $14 \%$ thought it would be fun, $41 \%$ were encouraged, asked, or told to participate: 
Parents wanted me to

Mum asked me

Got told to and said yes

It is important to note here that the counsellors, parents and students were informed that the programme was completely voluntary.

\section{How students felt about being asked to join Kiwi ACE}

Thirty five percent of students were excited and felt that it was a good thing to be asked to join Kiwi ACE, some of these comments included:

Really happy and excited

Sweet as, algud

I was unnecessary (crazily) excited.

Thirty five percent were not sure why they were asked, $17 \%$ thought that there was something wrong with them, for example:

I thought it was because I was depressed.

I must be retarded

Like I had problems

and $13 \%$ said they were "embarrassed" or "nervous" about being asked to be in the group.

\section{How Kiwi ACE participants were treated by their peers for being in the group}

Forty three percent of students did not tell their peers that they were attending Kiwi ACE and 26\% did not answer the question. Those students who did tell their peers received positive feedback such as:

They were like awesome I want to join

they said it would be cool

One student's friend was worried that being asked to be in the group meant that they had depression. 


\section{Group Dynamics}

\section{How well did Kiwi ACE participants get on with their peers and leaders in their group?}

When asked how well students got on with their peers in the group, 48\% said they got on "well" or "really well" with their peers, $35 \%$ said that they got on "ok" with their peers, and $17 \%$ rated their relationship just below "ok". When asked how well students got on with the leaders in the group, $57 \%$ said they got on "well" or "really well" with their leaders, $35 \%$ said that they got on "ok" with their leaders, and 8\% rated their relationship just below "ok". One student gave 2/7 for their relationship with their peers and leaders, all other scores were three or higher. Sixty one percent of students thought that the leaders were "effective" or "very effective" at running the group, 35\% thought that they were just "above average" or "average" at running the group and 4\% rated the leaders' effectiveness just below "average" at running the group (3 out of 7).

In the space where students were given the opportunity to write anything else about the people in their group, one student commented that their leader was boring, and eight students thanked the leaders, and some comments are given below as examples:

I just want to say that I really loved the leaders they really helped me and I would like to say "THANK YOU" so much!!

I would just like to say a huge thank you to the leaders for doing a good job in teaching us to have a better life.

Leaders from Kiwi ACE reported that the students got on well with them and each other and that the leaders got on with each other. Two leaders from different groups felt that they were not as effective at running the group as they would have liked, and gave themselves 3/7 whereas all of the other leaders rated themselves as 6 or 7. Most leaders expressed that over time their groups became quite cohesive and supportive, over time the students felt more comfortable in sharing. One leader did express that year 9 students were a bit too young and immature, and did not take it as seriously as they should have.

\section{Distraction and Participation}

One student was identified by two of their peers as a distraction to the rest of the group. Two students in another group were identified as a distraction to their group by two different peers. The leaders of these groups identified the same students mentioned by their 
peers as a distraction to the group. In one group, the student who was identified as a distraction left after the fourth session of the programme. The other two students improved slightly over time, however the leaders did find these students difficult to manage and reported that at times they hindered the progress of other students. Though they did acknowledge that the recruitment of individuals "at risk" usually results in the selection of students that evidence behavioural problems (associated with adolescent depressive symptoms).

\section{Review of Kiwi ACE Sessions in Content and Process}

\section{Leaders' Feedback on Sessions}

The following section is a brief overview of how leaders found the content and process of each session in Kiwi ACE. The first session was considered to be a good opener by leaders, though some groups were found to be more engaged than others in this session. In some groups there was already an element of trust and friendship, and in other groups it took longer for students to become comfortable enough to participate. The majority of leaders reported that they ran out of time in the session Thoughts and feelings, however there was good interaction among the students in the activities that were completed. In the session Thoughts are not facts, students enjoyed the role plays, but again, leaders felt pressured to get all activities completed in time. In the session Check the evidence, leaders noted that students started to challenge their own thinking and were able to apply realistic thinking in their own experiences. However, a few leaders reported that there was a lot of off-topic chatting.

According to all leaders, students found the session on Problem solving engaging and participated enthusiastically in all of the activities. Leaders reported that the session on Habits was received well by students, especially the games, and students found the idea of thinking errors interesting and wanted to learn more. One leader commented that students did not understand the concept of "triggers" and felt that it needed further explanation. For the session on Social skills, leaders reported that students generated insightful discussions and kept focused on the assigned tasks. Leaders reported that students enjoyed the role plays in the session on Assertiveness and in Negotiation and compromise and were able to relate to these skills and shared examples from their own lives. One leader commented that the majority of students in their group already displayed good assertiveness, negotiation, and compromise skills.

Leaders reported that the session on Self-esteem captured students' interest, especially the section on deep-down beliefs and students shared readily the actions they would take to 
boost self esteem. Some leaders commented that it involved too much reading. One leader noted that all students in the group struggled to say what they liked about themselves. In the review session, leaders commented that students demonstrated learning from the course, including students who had not participated readily in the past. Students expressed to the leaders that the discussions and role-plays were their favourite activities in the sessions, and some students were eager to take part in the programme again the following year.

\section{Comparison of Leaders' and Students' Ratings of Sessions}

The results from the students' feedback forms showed that Assertiveness and Check the Evidence were rated as the most enjoyable sessions in Kiwi ACE. Changing Habits and Thoughts are not Facts were rated as the most helpful sessions. Check the Evidence and Thoughts are not Facts were rated as the skills practiced the most. See table 14 for students' rankings of all the sessions.

The leaders' estimation of students' enjoyment, perceived helpfulness, and practice of Kiwi ACE did not entirely agree with what students reported. The leaders estimated that students found Problem solving and Negotiation and compromise to be the most enjoyable and helpful sessions. They also reported that Negotiation and compromise and check the evidence were the skills that students practiced the most.

Table 14. The means of students ratings on how much they enjoyed, found helpful, and practiced each session in Kiwi ACE

\begin{tabular}{llllll}
\hline Most Enjoyed & Means & Most Helpful & Means & Most Practiced & Means \\
\hline Assertiveness & 5.30 & Habits & 5.09 & Check evidence & 5.00 \\
Check evidence & 5.26 & Thoughts -facts & 5.05 & Thoughts -facts & 4.95 \\
Thoughts - facts & 5.13 & Check evidence & 5.04 & Thinking -feeling & 4.64 \\
Self esteem & 5.10 & Assertiveness & 5.04 & Assertiveness & 4.61 \\
Social skills & 5.09 & Stress & 4.96 & Habits & 4.57 \\
Habits & 5.09 & Self esteem & 4.95 & Self esteem & 4.52 \\
Thinking -feeling & 5.00 & Problem solving & 4.87 & Social skills & 4.43 \\
Problem solving & 4.91 & Thinking -feeling & 4.87 & Negotiation & 4.23 \\
Stress & 4.87 & Negotiation & 4.68 & Stress & 4.14 \\
Negotiation & 4.82 & Social skills & 4.61 & Problem solving & 4.09 \\
\hline
\end{tabular}




\section{Overall Assessment of Kiwi ACE by Leaders and Students}

\section{How did the students find the programme overall?}

Fifty two percent of students said they "liked" or "really liked" the programme, $43 \%$ indicated "it was ok" or just above "ok" (5/7), and 4\% rated the programme just under "it was ok" (3 out of 7). Seventy four percent of Kiwi ACE participants rated the helpfulness of the programme in the range "a little helpful" to "very helpful" and $26 \%$ scored the programme's helpfulness just below "it was a little helpful". When asked "how was the programme helpful?" twenty two percent of participants did not answer this question and $78 \%$ reported that it gave them skills that helped them in life; some students reported specific skills while others gave more general comments, and some examples include:

Learnt how to deal with my emotions learnt that other people have problems too Helped me lots with my social skills

I know how to deal with situations

\section{Did students continue to practice the skills learnt?}

Forty eight percent of participants reported that they still practiced some of the skills learnt in the different sessions at the end of the programme, while 38\% did not answer the question.

\section{Students' Suggestions of Improvements to the Programme}

When asked what students would change about the programme, 22\% of participants said that they wanted more fun, games, or treats, one student did not want the group to be in class time, $13 \%$ said they wanted more time and for it to not be so rushed, and the remainder $(30 \%)$ said that they would not change anything about the programme. In the space where students were given the opportunity to write anything else about the programme, one student said that one of their leaders was hard to talk to and six students commented that they enjoyed the programme or/and thanked the leaders for their help. Some examples are given below:

It was a very awesome and helpful learning experience. And I'm glad I was asked to join it. And I hope others next year get to have the same experience as me.

I enjoyed it \& liked getting to know people I wouldn't normally talk to 


\section{Strengths, Concerns and Improvements raised by the Leaders of Kiwi ACE}

The majority of the leaders expressed the view that Kiwi ACE had a positive impact on the students who participated and were generally happy with how it went in their group. The leaders felt that Kiwi ACE equipped students with useful strategies to cope with difficult situations and opened up more understanding to their own thoughts and feelings. However there were some concerns and issues that were raised by the leaders. The following section outlines concerns raised and improvements that leaders proposed about the programme.

\section{Structure}

The majority of the leaders felt that most sessions in the programme had too much content and as a result felt pressured to rush through it. It was suggested that one activity could be taken out of each session to make it possible to complete the sessions comfortably and cover the content thoroughly.

\section{Student Engagement}

Leaders felt that sessions could be more interactive, with more role-plays and games as these were effective methods of keeping students engaged. Some sessions were considered to be too reliant on the manual and had too many writing tasks.

\section{Appropriateness of Programme}

One leader commented that many of the sessions were not appropriate for students with learning difficulties. It was suggested that the sessions could be adapted to be more suitable for all levels of ability, or at the least include alternative activities that are predominately oral for students with literacy difficulties. On the other side of the spectrum, another leader commented that discussions in small groups were not uncommon for students in their school, the students already had many opportunities to be heard and already had many of the skills taught in the programme; this leader felt that the programme would be of more benefit to less privileged students.

\section{Homework}

As with PAL, the leaders in Kiwi ACE found that the majority of the students did not do their homework for most sessions. Also the majority of the leaders still called the to practice tasks homework. Some leaders found it difficult to continue to encourage students to do homework and felt quite despondent having to start sessions asking about homework. 


\section{Continuation of Kiwi ACE}

The leaders commented that the manual had many useful exercises that they will use again. Leaders also mentioned that many of their students expressed an interest in doing a similar programme the following year.

\section{PAL's Qualitative Results}

\section{Why Students Joined the Group}

Students gave one or more of the following reasons as to why they agreed to participate in PAL when they were initially asked to join: $38 \%$ of participants felt that the group might help them in some way and that they may learn something from participating, examples of these responses include:

\section{I thought it would give me confidence \\ Cause I thought it might help \\ Because I thought it would be a good learning experience.}

Twenty seven percent of students wanted to participate in PAL so that they could skip class, $24 \%$ thought it would be fun, $11 \%$ either said they did not know why they agreed to go, or stated that they simply just wanted to, $14 \%$ decided to go because they were encouraged or asked to participate, for example:

Because my mum thought it would be good for me

Because they asked if I want to be in it

I got choosen to join it LOL

Half of the students that were encouraged by someone else to join the group also mentioned that they thought it was a good idea:

Because my family and I agreed that this programme will help me Because it looked like fun and my parents said it would be good for me. 


\section{How students felt about being asked to join PAL}

The majority (57\%) of students felt "excited", "happy" or "special" when they were asked if they wanted to join the group and felt that it may help them, for example:

That this could help me get past/through tough situations

Ifelt good, a new opportunity

Some students thought that being selected meant that there was something wrong with them or that they were depressed (19\%), for example:

I thought I was dumb or something or I was in trouble

I thought I was depressed! Everybody did

Like I thought I must have issues and I was afraid to show/tell my parents because I didn't know what they would think

A small percentage were nervous about being asked to be in the programme (8\%) and the remaining (30\%) students reported that they either did not know how they were feeling or that they did not mind either way, for example:

Well I didn't really mind. So I just decided to.

I don't know

\section{How PAL participants were treated by their peers for being in the group}

Most students did not tell their friends that they were attending PAL (65\%). The students that did tell their friends were either encouraged by their peers to join the group, (11\%) for example:

were positive about it

Lucky to get out of class

or said their peers "laughed" $(11 \%)$ and some peers thought that they were asked to be in the group because they were depressed or there was something wrong with them (19\%), for example: 
they thought it was anger management class

\section{Yes, I am retarded and special needs}

they thought I was depressed

The students' answers mentioned above were expanded on in the discussion time. Most students reported that their peer's perception about the group and their own perception changed once the group was established. Most peers' negative responses such as "laughing" or thinking that group members were "depressed" stopped after the initial session. The students who thought they were being selected because they were depressed had mixed feelings, some were relieved to get help and others felt nervous or were confused because they did not think they were depressed. It is important to point out here at this juncture that when the school counsellors asked students to be in the group, they did their best to explain that students were invited because they might benefit from the programme, not because they were depressed. The counsellors were asked to emphasise that students who were depressed were not asked to be in the programme. (Students who scored in the clinical range for depression were seen alone by the counsellor). However many of the students who were asked to be in the programme would have been experiencing elevated depressive symptoms, as they were in the mild to moderate range, and it appears that some students were relieved to be identified but it made others feel nervous.

\section{Group Dynamics}

\section{How well did PAL participants get on with their peers and leaders in their group?}

When asked how well students got on with their peers in the group, 59\% said they got on "well" or "really well" with their peers and 32\% said that they got on "ok" with their peers and $9 \%$ rated their relationship just below "ok" (3 out of 7). When asked how well students got on with the leaders in the group, 51\% said they got on "well" or "really well" with their leaders and $46 \%$ said that they got on "ok" with their leaders, and $9 \%$ rated their relationship just below "ok" (3 out of 7). Sixty-five percent of students thought that the leaders were "effective" or "very effective" at running the group, 30\% thought that they were just above "average" or "average" at running the group, and 5\% rated the leaders effectiveness just below "average" at running the group (3 out of 7). It is notable that there were no scores under 3 for the relationships between peers or with leaders or for the effectiveness of leaders at running the programme. 
In the space where students were given the opportunity to write anything else about the people in their group, six students commented on the leaders and/or that they enjoyed the programme, and below are some examples:

Thanks a lot for having me on this course it was real exciting and I enjoyed it.

They were awesome. Very grateful to have been a part of this group! Got to know people more.

Leaders from PAL reported that the students got on well with them and each other, and felt that they were effective at running the group. The leaders also reported that they got on with each other and though teaching styles differed slightly there were no significant issues in working together.

\section{Distraction and Participation}

Two students from the same group were identified by each other as a distraction to their group. One student in another group was also identified as a distraction by a peer. Interestingly the leaders of these groups did not identify the same students mentioned by their peers as a distraction to the group. In all the groups the leaders identified one or two students that lacked motivation to participate in activities or presented with a negative attitude that hindered others, however all these students became more engaged over time.

The leaders from two groups reported that initially all students in the group struggled with contributing during discussion times, though as the students in the group got to know each other, they participated more readily. All leaders reported that the majority of students expressed the view that the friendships they made in the group was one of the best things about PAL.

\section{Review of PAL Sessions in Content and Process}

\section{Leader's Feedback on Sessions}

The following section is a brief overview of how leaders found each session in PAL. For the first session, leaders commented that the majority of the students were hesitant to share with each other at first, some groups took longer to engage than others, however all leaders thought that the activities in the first session helped students relax and get to know each other. According to all the leaders, the gratitude session was well received by students. One leader commented that the students' confidence grew as a result of sharing with others about the things for which they were grateful. Many of the leaders encouraged students to 
share what they were grateful for throughout the programme and found it was an effective way to engage students. The character strengths session was said to be quite complex by most leaders, many of them felt the terminology was too difficult for students. Some leaders observed that students drew pleasure and gained confidence from learning more about their strengths. The main comment from leaders in the savouring session was that the chocolate (an example of savouring) was enjoyed immensely, though in one group the girls were hesitant to eat it because they were worried about their weight. The leaders felt that the students benefitted from the savouring activities as it was something they could relate to and easily do.

The session on flow had a mixed response from leaders, some said that it was difficult for the students to grasp, others said that students really got a hold of the idea and could not wait to engage in more flow activities. One leader commented that the boys in the group gravitated to the concept of flow more readily. The problem solving session was said to be enjoyed by all, as it was very interactive and engaging. Leaders commented that most students contributed enthusiastically to this session. All leaders felt that the session on goal setting was quite challenging for students, though they also felt that it was a very helpful activity for them to do. The leaders found that goal setting demanded more individual attention and was not as intuitive for the students as the other sessions.

The session on acts of kindness received the highest number of positive comments from the leaders out of all the sessions. Leaders found that students related very well to this concept and they saw immediate, visible results that continued for weeks. Many acts of kindness were mentioned in the groups, and students were enthusiastic about doing the acts of kindness when they went home. Leaders commented that the assertiveness session was difficult for some students, and one parent did not appreciate their child "arguing" with them when their daughter attempted to practice her new assertive skills. Most groups enjoyed the role-plays, though the more reserved students found it quite challenging to participate. The negotiation and compromise session was found to have similar outcomes to assertiveness. Some leaders felt that the sessions on negotiation and compromise were teaching students skills they needed, others commented that many students already had these skills. The majority of leaders commented that the session on self-esteem involved too much heavy reading for the leaders and not enough activities. One leader commented that the self-esteem session should have come earlier so that there would have been more time to build on the issues raised. 
For the review session, leaders reported that the students demonstrated a good understanding of the topics learnt, and were creative in their presentations. Students reported that they enjoyed the discussions and role-plays the most out of all the activities. According to the leaders, students gave positive feedback about the programme, with many students insisting that they wanted to do it again.

\section{Comparison of Leaders' and Students' Ratings of Sessions}

The results from the students' feedback showed that Savouring and Character Strengths were rated as the most enjoyable sessions, Problem solving and Savouring were rated as the most helpful and Savouring and Gratitude were rated as the skills practiced the most. Goal Setting obtained the lowest score for all areas. Further discussion with the leaders and students revealed that the goal setting module was too much like school work for the students. Some leaders mentioned that goal setting was not intuitive for students and did not feel relevant to them. For students' ratings of all individual sessions see table 15.

Sometimes the leaders' estimation of how the sessions went for the students were congruent with what the students reported. The leaders estimated that the students enjoyed Acts of Kindness and Problem Solving the most out of all the sessions. The leaders also felt that the students found Acts of Kindness and Goal setting the most helpful and Acts of Kindness and Assertiveness as the most practiced skills learnt. What is important to note here is that students did rate Acts of Kindness quite high in all areas (see table 15 mean above 5 in all areas).

However, sometimes the students and leaders disagreed. Firstly the leaders' observation of students' disengagement, distractibility and silence often lead to an assumption that students were not retaining information or were not enjoying tasks. Sometimes this was the case, however it appears that for some sessions, where many of the leaders thought a session did not go well, or sessions were too difficult, students rated these same sessions quite high on enjoyment and helpfulness. A good example of this was with the character strengths session. Most of the leaders thought the language was too advanced and that the concept was too difficult to grasp, however the students rated the character strength session as one of the most enjoyable sessions of the programme and almost half (41\%) of the students could still remember what their character strength was 10 weeks after the session. The reverse was also true; in some sessions where leaders felt that the activities went really well, and everyone was contributing and engaged, the students did not always rate these sessions as the most enjoyable or helpful. For example, the leaders' comments on the 
problem solving session indicated that students were highly engaged and having a lot of fun, however the students rated this as one of the least enjoyable sessions (albeit one of the most helpful sessions).

Table 15 The means of students ratings on how much they enjoyed, found helpful and practiced each session in PAL

\begin{tabular}{llllll}
\hline Enjoyed the most & Means & Most helpful & Means & Practice most & Means \\
\hline Savouring & 5.75 & Problem solving & 5.29 & Savouring & 5.25 \\
Character strengths & 5.50 & Savouring & 5.25 & Gratitude & 5.18 \\
Assertiveness & 5.32 & Acts of kindness & 5.21 & Problem solving & 5.14 \\
Gratitude & 5.21 & Character strengths & 5.12 & Acts of kindness & 5.00 \\
Acts of kindness & 5.21 & Negotiation & 5.11 & Negotiation & 5.00 \\
Flow & 5.14 & Gratitude & 5.11 & Flow & 4.93 \\
Self esteem & 5.00 & Self esteem & 4.96 & Assertiveness & 4.79 \\
Negotiation & 4.89 & Assertiveness & 4.89 & Social skills & 4.75 \\
Social skills & 4.79 & Flow & 4.89 & Character strengths & 4.65 \\
Problem solving & 4.68 & Social skills & 4.82 & Self esteem & 4.56 \\
Goal setting & 4.43 & Goal setting & 4.36 & Goal setting & 4.11 \\
\hline
\end{tabular}




\section{Overall Assessment of PAL by Leaders and Students}

\section{How did the students find the programme overall?}

Sixty three percent of students said they "liked" or "really liked" the programme, $32 \%$ rated that "it was ok" or just above (5/7) and 5\% rated the programme just under "it was ok" (3 out of 7). Ninety two percent of PAL participants rated the helpfulness of the programme in the range "a little helpful" to "very helpful" and $8 \%$ scored the programmes helpfulness just below "it was a little helpful" (3 out of 7). No scores fell below three. When asked "how was the programme helpful?" $43 \%$ of participants did not answer this question and $43 \%$ reported that it gave them skills for everyday life - some students mentioned specific skills and others commented more generally, some examples include:

It's helped me communicate better and helped me deal with problems more better.

It helped me not to get in as many arguments with my dad.

Made my every day easier!

Additionally $14 \%$ of participants reported that it made them happier and more positive about life, for example:

It just made me feel good.

It taught me positive ways to look at situations

Made me happy

\section{Are students still practicing skills at the end of the 12 week programme?}

Forty three percent of participants reported that they still practice some of the skills learnt in the various sessions, 38\% did not answer the question and 19\% reported that they did not continue to practice any of the skills learnt in the programme. Interestingly one leader commented in discussion that often students did not realise when they were practicing the skills they learnt as it had become a part of their natural behaviour.

\section{Students' Suggestions of Improvements to the Programme}

When asked what students would change about the programme, $17 \%$ said that they wanted more fun, games or treats, $5 \%$ of students wanted a bigger room to have the programme in, $24 \%$ did not answer the question and the remainder (59\%) said that they would not change anything about the programme. In the space where students were given the 
opportunity to write anything else about the programme, one student said they found it boring because they missed out on subjects they liked and six students commented that they enjoyed the programme or/and thanked the leaders for their help, some comments are given below as examples:

\author{
Really enjoyed it, learn a lot \\ It was a great help! Thanks! \\ IT WAS AN AWESOME EXPERIENCE! Going to miss it A lot!
}

\title{
Strengths, Issues and Improvements Raised by the Leaders of PAL
}

On the whole leaders were happy with how PAL unfolded in their group and felt the programme produced positive outcomes in the students who participated. The leaders found that the programme gave students time to reflect on their lives and discuss issues. The leaders valued that PAL gave students a sense of community every week and an opportunity to express themselves. As mentioned earlier, one of the clearest positive outcomes was that students were able to develop new friendships and social connections. Positive changes in students' behaviour during the programme were noted by teachers, some teachers even mentioned these changes to the school dean. The following section outlines dominant concerns, improvements and strong points that leaders raised about the programme.

\section{Homework}

The leaders felt that the majority of the students were resistant to do the homework for each session. At the training leaders were asked to not label the take home tasks as homework but rather to call them activities to practice, or to try out a theory, to test out an idea to see if it worked, to experiment - and so forth. However it was discovered that the majority of the counsellors still called the take-home activities homework. There were a few students in different groups who made negative comments about homework and for the majority of sessions the counsellors reported that the students did not practice the take-home tasks. However the exceptions to this trend were the Gratitude and Acts of Kindness sessions: all leaders reported that many of the students in their group did these activities at home and enjoyed doing them. In the group, students shared willingly their acts of kindness and gratitude tasks they had done during the week, and some students continued to share these throughout the programme. 


\section{Student Engagement}

All the leaders found that when the activities involved breaking into smaller groups of two or three people, that this often brought about more participation and engagement, and that the group was easier to manage. The time of the day played a big part in students' engagement; most sessions were held in the morning so that students' concentration would be at its best, but this was not always possible due to the rotating nature of the class schedule at some schools. The leaders always noticed a decline in students' concentration and engagement in the afternoons. Also two schools in particular seemed to have a few students that often arrived late and disrupted the class.

Two leaders commented that the programme did not seem effective for a few individual students, and how they behaved detracted from the positive growth in other group members. Having more interactive activities was a common suggestion for helping students to become more engaged.

\section{Structure of the Programme}

The leaders felt that at times it was challenging to run the programme alongside all their other commitments at the school. They suggested making the programme shorter by having fewer topics. Another suggestion was to have fewer activities in any given session, so that one topic could be covered thoroughly because some sessions felt too rushed.

\section{Suicide}

Just before the Time 4 data collection (one year after the programme) a cluster youth suicide occurred in the Wellington region. A number of students across different PAL groups knew one or more of the students who had ended their life. The leaders involved were concerned for all students, especially those closest to those who had committed suicide and they reported a noticeable change in behaviour in the schools concerned. As a safety measure all students were seen by their school counsellor. The counsellors felt this may impact the students' responses on the questionnaire as it occurred not long before students completed the questionnaire at Time 4. (Unfortunately it was not possible to determine if this had impacted responses as it was difficult to establish what students had been effected and to what extent).

\section{Continuation of PAL}

The majority of the leaders wanted to continue to run PAL and to use some sessions from PAL in their individual work. The programme was a helpful new resource, it gave an alternative to CBT techniques, as not all students responded well to identifying cognitions. 
Additionally, it was found very useful that sessions stood by themselves, in the sense that students did not have participate in a previous session to make sense of a subsequent session. The leaders expressed that the programme held students interest and they could see the benefits of each session. Overall the school counsellors and psychologists enjoyed running the groups and felt that the majority of students developed valuable strategies and perspectives in dealing with life's challenges.

\section{PAL and Kiwi ACE}

Overall when comparing the responses of the students and leaders from both programmes, it appears that PAL had a more positive response. For example 52\% of participants from Kiwi ACE rated that they liked or really liked the programme, in contrast to $63 \%$ of PAL participants. In comparing how helpful students thought the programme was, $74 \%$ of Kiwi ACE participants thought it was helpful, compared to 92\% of PAL participants. Additionally leaders gave more positive feedback with PAL, though there were some issues, they found that students responded very well to the programme; whereas leaders from Kiwi ACE encountered more problems with student engagement. However, overall both programmes were well received by both leaders and students, and there were no issues that destroyed the integrity of running both programmes. 


\section{Chapter 4: Discussion}

The current study examined the efficacy of two school-based prevention programmes that sought to build resilience in 'at risk' year 9 students. Kiwi ACE used a CBT framework that aimed to decrease depressive symptoms. PAL consisted of strategies from positive psychology that aimed to increase students' wellbeing and positive outcomes. The three purposes of this study were: 1) to determine whether one or both of these programmes would obtain significant intervention effects; 2) to investigate whether these programmes would obtain different results for the positive and negative outcome measures; and 3) if so, which mechanisms explained these differential outcomes. As of this writing, no prevention programmes that target at-risk youth with a variety of intentional positive psychology activities aimed to cultivate positive emotions had been identified, so the current study filled an important gap in the literature.

The current study found that both programmes obtained significant intervention effects. Both programmes were found to decrease depression and increase psychological well-being. However, PAL also helped to increase other positive outcomes, including personal resilience, that Kiwi ACE did not. When comparing programmes, different outcomes were found as a result of the intervention, suggesting the programmes had different effects. Also mediational analysis revealed that there are many routes to well-being and that these are largely different to those leading to depression. All of these findings will be discussed below in detail in relation to the literature.

\section{Outcomes of the Kiwi ACE Intervention}

\section{Depression and Maladaptive Coping}

As predicted, and this was the main goal of this programme, Kiwi ACE helped to decrease participants' depressive symptoms and maladaptive coping up to one year after the programme. Prior trials of ACE (Kowalenko et al., 2005) and Kiwi ACE (Woods, 2006; 2011) have previously found a decrease in depression at follow up as well. Kowalenko et al. (2005) also found that ACE helped to decrease non-productive coping strategies (i.e. ignoring the problem, worrying about the problem, smoking or drinking to escape the problem) which is similar to the construct of maladaptive coping (i.e. ignoring the problem, smoking, drinking or taking drugs) used in the present study. Contrary to prediction, Kiwi ACE in the present study did not increase adaptive coping. This finding is not consistent with Kowalenko et al. (2005) who found that ACE helped to increase productive coping (i.e. problem solving, 
talking to others), a similar construct to adaptive coping (i.e. tried to solve the problem, talked to someone about the problem) used in the present study. This failure to increase adaptive coping does not mean that students did not gain positive outcomes, as Kiwi ACE did lead to increased psychological well-being (which will be discussed in more detail below). However, this finding seems to suggest that Kiwi ACE did not increase the specific strategies identified in the adaptive coping construct used here.

\section{Psychological Well-being}

As mentioned above, Kiwi ACE increased psychological well-being up to one year after the programme. Most CBT prevention programmes do not measure participants' psychological well-being as an outcome. The majority that have achieved well-being outcomes combined CBT with positive psychology strategies and therefore makes it difficult to distinguish which strategies caused the various outcomes (e.g. Rashid \& Anjum, 2008). One study did find that a CBT prevention programme increased well-being with adolescents, however, the study lacked a control group, did not examine 'at-risk' youth, and only obtained one post-test after the programme ended with no long-term follow-up tests (i.e. Ruini et al., 2006). The finding obtained with the present study significantly adds to the literature by showing that CBT programmes that target at-risk adolescents can not only lessen depressive symptoms but also contribute towards their psychological well-being. Although the dominant aim of CBT strategies is to prevent depression, in the present case it also managed to increase psychological well-being as well.

\section{Resilience}

On the other hand, incongruent with the previous finding, Kiwi ACE did not increase resilience as predicted. The results from PAL (see below) suggest that Kiwi ACE may not have provided adequate resources to support the development of resilience, that is, Kiwi ACE did not increase momentary happiness or adaptive coping as PAL did. Alternatively, it could be possible that Kiwi ACE helped to increase only one aspect of personal resilience, as Kiwi ACE helped to decrease depression through increasing environmental mastery.

Environmental mastery is similar to the resilient characteristic of self reliance (in the Resilience Scale) and sense of mastery (in the Resiliency Scales for Children and Adolescents). Yet Kiwi ACE had no discernible impact on the RS subscale self reliance. Looking at the differences between these two subscales, one explanation could be that Kiwi 
ACE helped to develop participants' ability to plan and organise their life in a way that is satisfying to them, rather than developing a belief in their own self efficacy ${ }^{27}$.

\section{Total Difficulties}

Total difficulties only obtained marginal significance, indicating that Kiwi ACE may have had some impact on lessening difficulties but not a large impact. Total difficulties included peer problems, conduct problems, hyperactivity, and emotional difficulties. The literature suggests that CBT helps to decrease these various difficulties, as they are associated with depression (Spirito et al., 2011). Although the relationship between Kiwi ACE and total difficulties was consistent in direction, and this direction was congruent with the literature, it did not show adequate strength to allow a strong statement that 'Kiwi Ace reduces total psychological difficulties'.

\section{The Mechanisms behind Kiwi ACE}

\section{Mediators between Kiwi ACE and Depressive Symptoms}

The prediction that maladaptive coping would mediate the relationship from Kiwi ACE to depressive symptoms was not supported. This proposed relationship makes sense theoretically; the non-confirmation here may be due to any number of possibilities: heterogeneity of the 'maladaptive coping' construct, inability of the Kiwi ACE intervention to make a difference on the present construct, or a weak relationship between maladaptive coping and depressive symptoms.

The current study identified one variable that manifested a mediational pathway from the CBT intervention to a decrease in depressive symptoms. Through exploratory analysis, environmental mastery was shown to mediate significantly the relationship from Kiwi ACE to depressive symptoms. It is sensible to suggest that the CBT strategies in Kiwi ACE may have helped students gain a sense of control over external factors, leading to a decrease in depressive symptoms. This finding is supported in the literature, as individuals low on environmental mastery have been shown to be more susceptible to depression (Abbott et al., 2006). Individuals high in environmental mastery have a greater sense of control over their

\footnotetext{
${ }^{27}$ Items used for environmental mastery were: The demands of everyday life often get me down, I am good at juggling my time so that I can fit everything in that needs to get done, I have difficulty arranging my life in a way that is satisfying to me, I am quite good at managing the many responsibilities of my daily life.
}

Items used for self reliance were: I usually manage one way or another, I feel that I can handle many things at a time, I can get through difficult times because I've experienced difficulty before, My belief in myself gets me through hard times, When I'm in a difficult situation, I can usually find my way out of it. 
lives and feel that they have the ability to create change and take advantage of opportunities (Ryff \& Singer, 2006). This finding with environmental mastery is unique to Kiwi ACE as it was unrelated to depression in the PAL intervention. Interventions seldom thoroughly investigate the mechanisms behind change, therefore this finding expands the literature by identifying one of the possible mechanisms in which CBT programmes like Kiwi ACE may decrease depression.

It was predicted that a reduction of total difficulties would mediate the relationship from Kiwi ACE to depression. This prediction was not supported, however this relationship achieved marginal significance, indicating that there may have been a weak relationship.

\section{Mediators between Kiwi ACE and Psychological Well-being}

An unexpected finding was that a decrease in emotional difficulties was related to an increase in well-being rather than a decrease in depression. Emotional difficulties involved items such as feeling: angry, unhappy, worried, scared, and nervous. In other words, participants experienced a decrease in negative affect. In the exploratory analyses that were run, emotional difficulties was found to mediate the relationship between Kiwi ACE and well-being, indicating that Kiwi ACE helped to increase well-being by decreasing participants' experiences of negative affect. A decrease in negative affect is usually associated with an increase in subjective happiness (Ed Diener \& Biswas-Diener, 2008; Snyder \& Lopez, 2005), rather than an increase in psychological well-being. However, research has found that individuals who experience a higher ratio of positive affect than negative affect are more likely to be in a state of 'flourishing' - a construct that includes psychological wellbeing (Fredrickson \& Losada, 2005). This empirical finding in the present study suggests that decreasing negative affect can create significant gains in psychological well-being.

In addition, anhedonia was found to mediate the relationship between Kiwi ACE and well-being. This finding suggests that although Kiwi ACE did not directly impact on participants' happiness, the programme did help to increase students' ability to find pleasure in things (lower anhedonia), which led in turn to increases in wellbeing. Anhedonia is a symptom of depression, so it was expected that anhedonia would decrease in response to the Kiwi ACE programme. Additionally, out of all the subscales of depression, it is logical that anhedonia was found to be (negatively) associated with well-being. The ability to find pleasure in things and events has been indicated as a determinant of well-being (Bryant \& Veroff, 2007; Fredrickson, 2001), so an intervention that is effective in reducing anhedonia should play a role in fostering happiness and well-being. Given the content of the Kiwi ACE 
intervention, it is not clear how it helped to increase students' ability to find pleasure. This finding may be the result of the shared sessions in both programmes (e.g. the development of social skills) or simply due to the positive impact of participating in a group activity. To establish more clarity, further research would be needed to identify which factors in both interventions helped to develop participants' ability to find more pleasure in everyday things.

\section{Outcomes of the PAL intervention}

\section{Psychological Well-being, Subjective Happiness, and Satisfaction with Life}

As predicted, PAL increased participants' scores on psychological well-being (PWB), satisfaction with life (SWL), and subjective happiness (SHS) up to one year after the programme. These findings are consistent with the broaden-and-build theory (Fredrickson, 1998; 2001), and the many interventions that have used intentional activities aimed at increasing these positive outcomes (Sin \& Lyubomirsky, 2009). Although there are studies that have found that separate strategies used in PAL have increased PWB, SWL, and SHS in adolescents, there has only been one study, undertaken by Savage (2011), that has used all these strategies in a combined programme. Savage (2011) measured subjective wellbeing (SWB), which included a measure of life satisfaction and optimism (a measure of negative and positive affect), but did not use Ryff's broader measure of psychological well-being which was used in the current study. She found that the SWB intervention was not related to the increase in positive affect post intervention. Rather, expectancy, child-rated alliance, and social self-efficacy were significantly related to the increase in positive affect. Her finding indicates that the participants' therapeutic alliance, expectancy of outcome and ability to express oneself were predictive of positive affect, rather than the content of the intervention. Though this current study did not control for these factors (expectancy, child-rated alliance, and social self-efficacy), the results suggest that the increase in happiness (similar to the construct of positive affect) in PAL may be due to the content. Firstly, the outcomes of PAL differed from the outcomes of the CBT intervention, in that PAL helped to increase happiness (gratitude, resilience, and life satisfaction), whereas Kiwi ACE did not show these additional benefits. This differential result may be indicating that it was the content of the programme that increased happiness. Additionally, exploratory mediational analysis revealed that gratitude helped to increase happiness in PAL which suggests that a specific strategy (the gratitude activities) used in PAL helped to increase happiness. Though factors such as expectancy and social self-efficacy would have most likely impacted the participants' outcome, the present author suggests that the strategies in the programmes may have 
determined some of the change in participants.

Nevertheless, Savage (2011) did find that the increase in life satisfaction after the programme was associated with the SWB intervention at the four month follow up. The current study found that PAL predicted an increase in life satisfaction up to one year after the programme. Savage used a four month follow-up test, whereas the current study was able to document that these types of interventions can positively impact individuals up to one year later. The current finding builds on and is congruent with prior research, but it demonstrates greater longevity in the development of a capacity for positive emotions than previous studies. Also, PAL demonstrated a general effect, namely leading to increased psychological well-being and happiness in addition to increased satisfaction with life.

\section{Gratitude}

As predicted, PAL helped to increase participants' gratitude scores up to one year after the programme. This finding is consistent with prior research that has found an increase in youth gratitude scores after a gratitude intervention, specifically gratitude journals and gratitude letters (Froh et al., 2009). In PAL, one session was dedicated to increasing gratitude; it involved various gratitude activities including: writing a letter of gratitude to someone, doing something for a peer or friend, and discussing three things for which they were grateful. In addition, during the following week students were encouraged to list three things daily that they were grateful for and throughout the following weeks students were asked to share things for which they were grateful. It is important to note that Froh and colleagues (2009) found that only students low in positive affect had increases in gratitude, which would have been true of the at-risk students included in the present study as well.

However, there are some obvious differences between Froh et al.’s (2009) intervention and the current study. Firstly, the current study's gratitude intervention was one of many modules contained in the overall PAL programme, therefore it cannot be said with certainty that the gratitude intervention by itself increased gratitude. Secondly, Froh et al.'s (2009) study was enacted over five days, with 10-15 min sessions each day, and there was a single 2-month follow-up. The current study lasted for 12 weeks, with an hour dedicated to the gratitude session, and it measured gratitude scores 6 months and one year later. It can be argued that the present intervention constituted a more robust teaching module and a more thorough test of its long-term effects than has previously been done in the literature. Froh and colleagues are some of the few researches that have looked at the effects of gratitude interventions for adolescents. Until now there have been no studies that have investigated if 
gratitude can be increased in at-risk adolescents. The current finding demonstrates not only that gratitude can be increased in at-risk adolescents, but that gratitude scores can remain elevated up to one year after intervention. This result has important implications for interventions as gratitude has been associated with an array of positive outcomes which will be discussed in more detail below.

\section{Resilience}

As predicted, PAL helped to increase participants' resilience scores up to one year after the programme. This finding is consistent with the broaden-and-build theory (Fredrickson, 1998; 2001) and the growing research that indicates building a capacity for positive emotions enhances resilience (Cohn et al., 2009; Folkman \& Moskowitz, 2004; Ong et al., 2006; Tugade \& Fredrickson, 2004). A core component of resilience is the ability to use effective emotional regulation strategies, especially those that use positive emotions to buffer negative experiences (Davidson, 2000; Tugade \& Fredrickson, 2007). Though emotional regulation was not directly taught or discussed in PAL, individuals were encouraged to use positive emotions as a way of approaching challenging circumstances. For example, students were asked to consider how they might use gratitude when things go wrong and to test it out to see how it could help. Also some of the specific activities used in PAL have been shown to be predictive of resilience, such as increasing flow (Schmidt, 2003) and goal setting (Masten et al., 2004).

It is important to note that most positive psychology interventions measure wellbeing and happiness as an outcome, and do not directly measure resilience as an outcome. Interventions usually measure the assets or the resilient behaviour they are attempting to increase, but it is rare for interventions to use scales such as the RS as an outcome. This approach is true for resilience programmes as they usually measure resilience as a process not as an outcome.

However, one rare study did measure personal resilience in order to gauge if an Outward Bound course (an adventure course) would enhance resilience (Neill \& Dias, 2001). The aim was to increase resilience through the physical, emotional, and social challenges faced during the programme. The study found a significant increase in participants' scores on the RS in comparison to the control group post-intervention. The current study's findings are consistent with Neill and Dias' (2001) findings, and both suggest that it is possible to increase personal resilience through intervention. The current study suggests that that personal resilience can be enhanced. Additionally, PAL increased resilience and Kiwi ACE did not, 
and this disparity indicates that the increase of resilience in PAL participants may be attributed to the content in that programme. By measuring personal resilience this study has helped to inform interventions in ways that other resilience interventions have not yet been able to do.

\section{Depressive Symptoms and Difficulties}

As predicted, PAL helped to decrease participants' depressive symptoms up to one year after the programme. This finding is consistent with the outcomes of positive psychology interventions with depressed and non-depressed populations which have shown significant decreases in depressive symptoms (Layous et al., 2011; Norrish \& Vella-Brodrick, 2009; Seligman, Ernst, Gillham, Reivich, \& Linkins, 2009; Sin \& Lyubomirsky, 2009). However, PAL is one of the first prevention programmes using a combination of positive psychology strategies that has been shown to decrease depressive symptoms up to one year after the programme.

Exploratory analysis also revealed that PAL helped to lessen self-reported difficulties (i.e. peer problems, conduct problems, hyperactivity, and emotional difficulties). This finding is consistent with research that indicates positive emotions help to lessen the impact of negative experiences and emotions (Epel et al., 1998; Fredrickson, et al., 2000; 2003; Fredrickson \& Levenson, 1998; McEwen, 1998). The strategies used in PAL have also been indicated in previous research as contributing to social resources, decreases in anxiety and increases in engagement. For example gratitude has been shown to promote pro-social behaviour, to maintain positive relationships (Algoe et al., 2008) and to reduce worry (Geraghty et al., 2010); which could have helped to lessen the difficulties reported in PAL participants. Kindness has also been shown to promote relationships and pro-social behaviour (Seligman et al., 2009; Smith, 2001; Souza \& Mclean, 2012). Additionally, savouring has been shown to lessen internalising and externalising problems (Grentzler et al., 2012). This current finding indicates that PAL helped to lessen the psychological difficulties experienced by participants up to one year after the programme. This finding supports and extends previous research by implicating that building a capacity for positive emotions (gratitude, kindness, savouring) helps to lessen the impact and experience of difficulties. 


\section{Mechanisms behind the PAL Intervention}

\section{Mediators between PAL and Well-being}

As predicted, happiness functioned as a mediator between PAL and psychological well-being. This finding is consistent with the broaden-and-build theory that positive emotions help to build resources (Fredrickson, 1998; 2001). Fredrickson's theory proposes that positive emotions expand cognitive pathways, which encourages an individual to consider a broader range of options that over time build helpful and adaptive resources. Although researchers, especially those from the field of positive psychology, have suggested that it is the positive emotions from interventions that build wellbeing, there has been no investigation to validate this theory. The current finding indicates that the intentional activities in PAL helped to increase students' happiness, which then led to build internal resources such as autonomy, self-acceptance, and the ability to make positive relationships with others.

As predicted, gratitude functioned as a mediator between PAL and psychological well-being. This finding adds further support to the broaden-and-build theory by showing that enhancing a specific positive emotion like gratitude can lead to increases in psychological well-being. The pathway from gratitude to psychological well-being has been discussed and supported in the literature. Gratitude has been associated with physical health (Emmons \& McCullough, 2003), sleep quality and duration (Wood et al., 2009), social integration (Froh et al., 2010), and positive relationships (Algoe et al., 2008). Interventions have found that intentional gratitude activities increase subjective wellbeing and school enjoyment in adolescence (Froh et al., 2008; Froh et al., 2009). However, these studies have not measured psychological well-being. Additionally, there have been no interventions, until now, that investigated whether an increase in gratitude helps to increase psychological well-being. The current finding indicates that gratitude is a helpful emotion that promotes mental health, and supports the use of gratitude activities in interventions targeting at-risk youth.

Exploratory mediational analysis helped to reveal two more pathways from PAL to psychological well-being. Anhedonia and meaning were found to function as separate mediators. As discussed above, anhedonia was also found to function as a mediator between Kiwi ACE and well-being, indicating this relationship was not unique to either of the two programmes. Although there is a possibility that anhedonia was decreased through different routes in each programme, this cannot be established with the available data. Flow and savouring activities, which were used in PAL, have been shown in the literature to help 
individuals find more pleasure in life. Unfortunately it was not possible to measure these two constructs in the pretest or outcome assessments.

Meaning, on the other hand, was only found as a mediator in PAL. Meaning is a subscale of resilience, and is defined as something to live for. This finding is consistent with research regarding the associations found with meaning and different aspects of mental health (Tennen \& Affleck, 2002). Individuals who are able to find meaning in their everyday lives despite challenging circumstances are less likely to experience feelings of hopelessness and despair (e.g. Tennen \& Affleck, 2002). Additionally, infusing meaning into ordinary daily events can help to offset negative situations and help generate gratefulness and optimism (Folkman \& Moskowitz, 2000). PAL aimed to increase meaning in participants through all of the intentional activities incorporated in the programme. For example, the session on goal setting endeavoured to help participants work towards intrinsic goals which fosters the development of personal meaning and purpose (Cantor, 1990; Sheldon et al., 2002). In the same manner, developing savouring, flow and kindness help to develop personal meaning and lead to enhanced wellbeing. The current finding suggests that PAL helped to increase participants' personal meaning, which then led to gains in psychological well-being. This finding adds to the literature by revealing an important new pathway that leads to psychological well-being from a positive psychology intervention.

\section{Mediators between PAL and Resilience}

Exploratory analyses revealed that three variables functioned separately as mediators between PAL and resilience: happiness, satisfaction with life, and gratitude. This cluster of findings is consistent with research that proposes that positive emotions help to build resilience (Cohn et al., 2009; Ong et al., 2006; Tugade \& Fredrickson, 2004). A longitudinal study by Cohn et al., (2009) found that positive emotions mediated an increase in resilience, but contrary to this current study, life satisfaction did not. Cohn et al. (2009) suggested that it was the in the moment positive emotions that build resilience, not necessarily having satisfaction in life. However, the current study suggests that being satisfied in life also helps to build resilience. This inconsistency could be due to the differences between the studies. Cohn et al. (2009) examined these variables in university students over one month, whereas this current study was an intervention with at-risk adolescents over one year. One explanation could be that Cohn and colleagues did not allow enough time to see the effects of SWL on resilience. Another explanation could be that in adolescence, SWL has a different relationship to resilience, especially with at-risk adolescents. Additionally, if students were low on 
satisfaction with life, then increasing their satisfaction may have created gains in resilience. Further research is needed to replicate these relationships over different time periods and in different samples of participants.

Although interventions have not previously investigated the relationship between gratitude and resilience, the present research suggests that gratitude may promote resilience by cultivating an appreciative attitude toward life that endures even while one is experiencing difficult circumstances (Fredrickson et al., 2003).The current results indicate that PAL helped to increase resilience by enhancing positive emotions, especially gratitude.

\section{Mediators between PAL and Depression}

Exploratory analyses revealed that PAL helped to decrease depressive scores by increasing gratitude, meaning, and satisfaction with life. These findings are consistent with research that has shown a strong association between depression and a lack of personal meaning (e.g. Tennen \& Affleck, 2002) and diminished satisfaction with life (e.g. Schimmack, Oishi, Furr, \& Funder, 2004). One study found that positive emotions, including gratitude, helped to protect individuals against developing depressive symptoms (Fredrickson et al., 2003). Also gratitude interventions have been found to decrease negative affect in adolescents (Froh et al., 2008) and depressive symptoms in adults (Toepfer et al., 2011). Yet no studies have searched for a mediating relationship for these positive variables between an intervention and subsequent depressive symptoms because most researchers tend to examine variables of a particular valence (e.g. negative mediators for negative outcomes). The current findings add to the literature by showing how a specific intervention helped to decrease depression in participants by increasing a number of positive attributes and dynamics.

\section{Comparing the Kiwi ACE and PAL Interventions}

Comparing the results from both programmes, it appears that PAL lead to additional positive outcomes. On the negative side of the equation, Kiwi ACE decreased both maladaptive coping and depressive symptoms, whereas PAL decreased depressive symptoms and self-reported difficulties (peer problems, conduct problems, hyperactivity, and emotional difficulties). The results reflect the dominant focus of Kiwi ACE, which is to decrease maladaptive beliefs and behaviour. PAL's dominant focus, on the other hand, was to increase positive outcomes, though interestingly still managed to decrease depression and difficulties. PAL increased all the positive variables measured (with the exception of adaptive coping), and Kiwi ACE only increased psychological well-being. The fact that PAL helped to increase 
more positive outcomes than Kiwi ACE is most likely due to the content differences between the programmes. Kiwi ACE aimed to help students choose more realistic thoughts in order to decrease negative emotions and change maladaptive beliefs. PAL, aimed to increase a capacity for positive emotions, and taught strategies not only to increase positive emotions but also how to use them in difficult times. The finding that Kiwi ACE increased psychological wellbeing specifically by decreasing the intensity and frequency of participants' negative emotions provides further support for the contention that it was the content in the programmes that led to the outcomes. It is interesting and important to note that PAL helped to increase well-being through a number of routes, including increasing students' gratitude, happiness, and their meaning in life. Both programmes helped to increase wellbeing through helping students find pleasure in everyday life (decreased anhedonia). This finding indicates a shared pathway to well-being, and may be a result of the shared structure and content of the programmes. For example, both programmes may have helped students gain stronger social networks and social skills by encouraging group participation and interpersonal sharing. The qualitative results suggest that both programmes facilitated positive relations with others, and students found this to be one of the best things about the programme.

In PAL, finding meaning in life (a subscale of the Resilience Scale), was shown to be a key pathway to decreasing depression and increasing well-being in participants. This result suggests that building personal resilience, especially in regards to helping students find personal meaning in their lives, promotes psychological well-being and helps to decrease depressive symptoms. Additionally, the results indicate that PAL helped students to develop strategies that led to a variety of positive outcomes and decreased depressive symptoms, suggesting that the participants acquired a depth and variety of resources to draw on when faced with challenges. This development of resources could explain why PAL helped to increase personal resilience, and why Kiwi ACE did not. Conversely, if we were to view resilience as a process, then it could be suggested that Kiwi ACE developed students' resilience against developing depression over one year's time. It might be more accurate to state that Kiwi ACE helped to decrease depressive symptoms by developing students' ability to manage their cognitions, emotions, and interpersonal relationships. Though PAL also helped to increase environmental mastery, it was not associated with students' decreased depression. This result indicates that mastery was a dominant mechanism in Kiwi ACE that helped to bring about change, whereas in PAL, mastery was more of an outcome, and other factors (i.e. gratitude, meaning, and satisfaction with life) were more instrumental in bringing 
about change. Overall, gratitude toward others and life in general increased among students who participated in PAL. These improvements helped to develop a stronger sense of self and helped to lead to a decrease in their interpersonal problems, low mood, and other symptoms of depression (measured with the CDI). When comparing future use of these two interventions, PAL would be the most logical choice as it decreased depression equally with Kiwi ACE, but it improved the positive side of the equation significantly more than Kiwi ACE.

\section{Discussion of Qualitative Findings}

It was important to conduct qualitative research to investigate if there were other issues or dynamics that the empirical scales did not identify, or if there were any confounding factors that may have contaminated the programme. The empirical findings tell us whether students benefited from the programme, but it is also important to establish if students enjoyed the programme. A programme that participants disliked is unlikely to be widely taken up both schools and institutions. It was imperative to ask leaders and teachers-in addition to the young people themselves - how they thought the participants were affected by the programmes. Additionally, this study was interested in future improvements both for the programmes' efficacy and student engagement.

Due to the stigma that is sometimes associated with depression and indicated programmes, there was concern that students may have felt singled out, become bullied, or put down by their peers for being associated with a 'special' programme. The results indicated that this was generally not the case. Though some students were somewhat concerned as to why they were invited to participate in a programme, it appeared that their fears soon dissipated. While some students encountered negative views, on a whole, peers thought that the participants were fortunate to be able to miss class and participate in more enjoyable activities.

The feedback suggests that the majority of groups got along quite well together. A few issues arose in some groups, however many of these were resolved and did not seem to pose any serious problems. Rather, it seemed that both programmes facilitated positive relations among the participants, and consequently the groups tended to function very well. The majority of students reported that they truly enjoyed getting to know their peers and that it was one of the best things about the programme.

All sessions in both programmes received average-to-high evaluation scores, and there seemed to be no significant issues with specific sessions. Both students and leaders 
from both programmes felt that some of the sessions were rushed as there was too much content to cover in the amount of time available. It was suggested that both programmes needed to have less content to cover and more interactive activities. Leaders from both programmes felt that students did not practice the activities assigned during the week. The empirical results indicate that though students may not have practiced the taught skills in their own time, the sessions still managed to have an impact on their mental health. On the other hand, leaders reported that in some PAL sessions students became very engaged in the practice tasks. Engagement on a whole appeared higher for PAL; participants in PAL rated most sessions higher than the participants in Kiwi ACE. Also, a higher percentage of PAL participants reported that they enjoyed the programme and reported more positive comments on a whole compared to Kiwi ACE. The difference in engagement may account for some of the differences in the outcomes, namely PAL's advantage in positive outcomes. Superior engagement in PAL may have contributed to the increase in happiness, satisfaction with life, and gratitude. This possibility is logical when considering the content of the programmes. Much of Kiwi ACE focuses on the consequences of negative thoughts and beliefs, whereas the content of PAL focuses on more enjoyable aspects such as students' strengths, activities they enjoy, and things for which they are grateful. Positive changes in students' behaviour were noted more readily by teachers, leaders, and students in PAL compared to Kiwi ACE. However, both programmes were well received by students and leaders and many of the students expressed their gratitude for being able to participate in a programme. Limitations

It is important to take into account the limitations of this study when reflecting on the results and conclusions drawn. The limitations to this study included: the small sample size, the use of self-report measures, length of the questionnaire, potential social desirability bias, influential environmental factors, and inability to control for all confounding variables.

The sample size of the current study was small $(\mathrm{N}=134)$, particularly in terms of analyses comparing the programme groups to the control group; Kiwi ACE ( $N=27)$, PAL ( $N$ =38). Also, PAL and Kiwi ACE did not have an equal number of participants; the relatively smaller sample size in Kiwi ACE may have not provided sufficient power to detect statistically significant between-group differences on a par with PAL. Additionally, with a small sample size, it was difficult to ensure a representative distribution of the population in all groups. Propensity matching was used to help achieve equal distribution between groups and to ensure that differences that were found were due to membership in control or treatment, and not other variables such as gender, ethnicity, baseline depression, well-being 
or other baseline scores. The current study attempted to include a representative sample of gender and ethnicity in New Zealand, including Māori, New Zealand European, Pacific Islander, and NZ Asian students. Also, care was taken to include a representative sample of schools, including public and private, religious and non-religious, rural and urban, high and low decile, and co-ed and single-sex schools. Schools that participated were predominately from the Wellington region, which could have biased the outcomes and made the findings less generalisable. However, in an attempt to increase generalisability, schools located as far as Masterton and Whanganui were included (both of these towns are small and rural). Although this study made the best efforts to obtain a diverse and representative sample, it is important to recognise that the study's findings may still not be equally applicable across all groups due to the small sample size.

The findings of the current study relied on self-reported data. Literature has found limitations to relying on self-reported data, including have no independent verification, false attribution of events, influence of previous questions, and the effects of affective state and events on answers to questions (e.g. Nisbett \& Wilson, 1977). In awareness of this, the current study took care to use measures that have been shown to be reliable and valid. Additionally, recent research suggests that subjective instruments measure individual wellbeing as studies have repeatedly showed that subjective and non self-report measures of wellbeing converge (Sandvik, Diener, \& Seidlitz, 2009). Schools were visited, and interviews with the leaders were conducted to establish if the leaders witnessed a positive change in the students and if there were any issues or confounding factors that needed to be addressed. Ideally, it would have been helpful to interview the parents of the participants, however it was not possible to do this due to time, and resource constraints.

The questionnaire used for assessment was lengthy as many scales were included for assessment. Towards the end of completing the questionnaire it was apparent that some students became bored. This phenomenon was evident through teachers' comments about students becoming restless and it was noted that some students' writing or marks became more illegible on the last few pages. In some cases, students left blank pages or started to make symmetric patterns with the answers on the sheet. Data cleaning removed questionnaires with numerous missing items and questionnaires where it was obvious students were not answering the questions seriously. Reverse-scored items helped to identify illegitimate questionnaires. Fortunately, students in the programme took more care at filling out the questionnaire, however, this raised the issue as to whether students in the programme were giving answers that the leaders wanted to receive. Social desirability bias was not 
controlled for, however, the questionnaire did use predominately forced-choice and randomised items, two techniques that have been shown to reduce social desirability bias (Nederhof, 1985).

The nature of the rooms used and the time of day the programme is run are important factors that can influence student engagement (Yalom, 1995). Leaders in this study were asked to run the programme in the morning, in a good-sized room, and to make the setting comfortable and informal. However, due to timetable scheduling, it was not possible for all schools to run the programme in the morning, and some schools had to rotate each week and have one session in the morning and the next session in the afternoon. Additionally, some schools had to conduct the programme in different sized rooms, very big or small because more appropriate room was not always available.

There were also other factors that may have influenced students' outcomes that could not be controlled. One example of this was the double suicide that took place in the Wellington region just weeks before some students completed the final follow-up assessment. It was not possible to identify all of the students who were impacted, although it was suspected that many of the students participating in PAL were affected.

Finally it is important to note that conclusions drawn from this study may only be appropriate for these interventions taking place in a school environment, with at-risk 13-14 year olds.

\section{Implications and Future Research}

This current study's findings have significant implications for prevention programmes, therapy, and research on resilience and mental health. The present results should be taken cautiously given the nature of the stated limitations, but the results still indicate grounds for replication and further research. The current study's findings lead to four main implications. Firstly, they indicate that prevention programmes should not only aim to decrease depressive symptoms but aim to increase a capacity for positive emotions and to enhance psychological well-being. Secondly, the findings suggest that there are different routes to enhancing psychological well-being and these are largely different to those that lead to a decrease in depression. Consequently, since different strategies in interventions lead to different outcomes, one should choose wisely in order to maximise the desired outcome. Thirdly, the results imply that personal resilience is not enhanced through strategies predominantly targeted at decreasing maladaptive behaviours or negative emotions, but by enhancing positive emotions through intentional activities. Finally, PAL helped to increase 
personal resilience, and therefore may have helped to create more robust and permanent effects than Kiwi ACE.

As discussed at the beginning of this chapter, PAL obtained more positive outcomes compared to Kiwi ACE, and it revealed more mechanisms of change that led to increases in well-being and decreases in depression. Unlike Kiwi ACE, PAL also helped to increase personal resilience, and the positive outcomes that PAL helped to increase worked as mechanisms to promote resilience. These findings imply that it was the content of PAL that brought about the positive outcomes, not only because Kiwi ACE did not obtain the same level of positive outcomes, but because of the strategies, content, and the aim of PAL. The aim for the strategies of PAL is to build personal resources that will assist the participant when they are faced with challenges. The findings suggest that aiming to increase well-being in at-risk adolescents is an equally effective, if not a more effective strategy, in prevention programmes designed to reduce depressive symptoms. Furthermore, it implies that using positive emotions as tools in times of stress is just as effective as directly changing 'unrealistic' thought patterns in order to reduce depression. This is not to say that PAL does not change thought patterns, rather, it may indirectly do so. Further research is needed to reveal if there are differences in underlying mechanisms behind change (some of these possible mechanisms are discussed below). Though other interventions have looked at the combination of CBT and these intentional activities, it is important to first assess what different strategies can specifically accomplish and how they might do so. The present findings indicate that the strategies used in PAL are useful on their own in prevention programmes, without CBT. The findings from PAL have useful ramifications for practitioners, especially school counsellors. PAL provides an alternate resource to CBT, as CBT is the dominant strategy used for the treatment and prevention of depressive symptoms. Results also imply that PAL may be easier to use and is more engaging than CBT. There is less explanation needed and components of each session can be used out of sequence or completely on their own. Also there are times when it is not possible to use CBT due to time constraints, student learning difficulties, or it may have been used in the past and had limited apparent success. Additionally, as the qualitative results suggest, early adolescents are likely to relate better to PAL and find it more stimulating.

The findings from PAL suggest that it is an effective tool to use across ethnicities in New Zealand, though further replications would be preferable with larger sample sizes to confirm this assumption. The implementation of programmes similar to PAL has the potential to significantly impact the high rates of depression in New Zealand. One method of 
implementation is to incorporate some of the principles and activities used in PAL into New Zealand school curriculums, as Martin Seligman has started to do already in Geelong Grammar School in Australia. Results from Seligman's assessment of this project are still underway; however, like the present study's results, their preliminary results look very promising.

Future research could also investigate if strategies used in PAL would be effective for treatment of mental illness and prevention of other types of serious psychological disorders (such as panic disorders, Obsessive Compulsive Disorder, and Major Depressive Disorder). As discussed in the introduction, some clinical case studies have started to include specific intentional activities to assist therapy. If more research were to find positive results, this may indicate that these intentional activities should take a more central role in individualised protocols. It would also be beneficial to know the additional effects of the positive outcomes gained in PAL. Research suggests that gratitude, happiness, and satisfaction with life are associated with many other positive outcomes. For example it would be interesting to know if these effects impacted their success at school, in relationships, and in their future choices.

Comparing the mediational paths in both Kiwi ACE and PAL that lead to the depression and well-being outcomes revealed some interesting implications, predominantly, that in future research and interventions, depression and well-being should be examined jointly. Getting to well-being and depression seem to involve different routes, in that different mediational variables affected one construct but not the other. To establish more clarity, further research would be needed to look at what factors in these types of interventions lead to the key mediational variables (i.e. mastery, meaning, negative affect, anhedonia, gratitude), and then in turn, to different outcomes. With PAL, it might be assumed that the gratitude session helped to increase gratitude, however it could be possible that other sessions such as kindness also had a significant impact on gratitude. It would be helpful if future interventions measured outcomes after each session and compared these to other sessions. The current study was not able to do this due to time constraints of each session. Also there are disadvantages to testing students after each session, namely, overexposure to assessment questionnaires can lead to fatigue and boredom. It would also be of interest to investigate if some routes are more sustainable than others, and if different individuals use different routes. The more robust results from PAL might reflect the variety of strategies offered in the programme that an individual could use and make their own compared to Kiwi ACE. The breadth and depth of strategies taught in PAL may have increased the odds that individuals would find a strategy that would work for them. 
The finding that resilience was increased through PAL and not through Kiwi ACE has several possible significant implications. This result implies that personal resilience is not enhanced through strategies predominantly targeted at decreasing maladaptive behaviours or negative emotions, but by enhancing positive emotions through intentional activities. This suggestion must be taken with caution, however, as there are many constructs that measure personal resilience, and it is possible that this study only captured one aspect of personal resilience. However, it appears that in regards to the RS measure of resilience, only PAL had an effect. Also, the results suggest that using positive emotions, such as gratitude and savouring, when faced with difficult circumstances is an essential component of personal resilience. An increase in personal resilience implies the development of characteristics that will enable participants to be more resourceful and flexible in the face of adversity. Due to the nature of personal resilience, this finding implies that PAL may have helped to create a more robust and permanent effect than Kiwi ACE. If PAL helped to increase personal resilience, then it is possible that it made more sustainable changes than the changes created by Kiwi ACE. However, this hypothesis requires further investigation. It needs to be noted that if personal resilience can increase, it can also decrease, also there is nothing to suggest that Kiwi ACE did not make sustainable changes. It would be helpful if future research would investigate the long-term effects, from adolescence into adulthood, of these different strategies and their association with personal resilience. Much more research is needed to help establish the determinants and outcomes of increasing personal resilience in intervention.

\section{Conclusion}

The current study builds on past research that has indicated interventions aimed at building a capacity for positive emotions lead to psychological well-being, resilience and decreases in depressive symptoms. The results obtained from the PAL intervention demonstrate that strategies that have come out of positive psychology research are effective prevention tools to use with at-risk adolescents. The outcomes from the programme are promising and add to current literature on the lasting effects of positive emotions and their function in building future resources. The understanding of personal resilience has been further developed, finding that it can be enhanced through building a capacity for positive emotions. The current study highlights the importance of focusing not only on dysfunction but on the strengths and healthy functioning of individuals in order to bring about positive change. Also, it reveals the many routes to well-being and positive outcomes in at-risk 
adolescents. The strategies in PAL are a helpful resource; they can be used as an alternative or in combination with CBT strategies that are currently being used in prevention. The current study has significant implications that can help to inform interventions, therapy and mental health research.

Much more research is needed in the area of positive human functioning and how this can inform dysfunction or those at risk of psychological difficulties later in life. However, the current study has found significant inroads into the vast area of psychological health that is still relatively untouched in the literature. 


\section{References}

Abbott, R. A., Ploubidis, G. B., Huppert, F. A., Kuh, D., Wadsworth, M. E. J., \& Croudace, T. J. (2006). Psychometric evaluation and predictive validity of Ryff's psychological well-being items in a UK birth cohort sample of women. Health and quality of life outcomes, 4, 76. doi:10.1186/1477-7525-4-76

Abela, J. R. Z., \& Hankin, B. L. (2008). Cognitive vulnerability to depression in children and adolescents: a developmental psychopathology approach. In J. R. Z. Abela \& B. L. Hankin (Eds.), Handbook of depression in children and adolescents (pp. 35-78). New York: Guilford Press.

Adlai-Gail, W. (1994). Exploring the autotelic personality. Unpublished doctoral dissertation, University of Chicago

Adler, M. G., \& Fagley, N. S. (2005). Appreciation: Individual differences in finding value and meaning as a unique predictor of subjective well-being. Journal of Personality, 73, 79-114.

Adolescent Health Research Group. (2008). Youth'07: The health and wellbeing of secondary school students in New Zealand. Initial findings. Auckland, New Zealand: The University of Auckland. Retrieved March 27, 2009 from http://www. youth2000.ac.nz/publications /reports-1142.htm

Ahern, N. R., Kiehl, E. M., Sole, M. Lou, \& Byers, J. (2006). A review of instruments measur-ing resilience. Issues in comprehensive pediatric nursing, 29(2), 103-25. doi:10.1080/014 60860600677643

Algoe, S.B., Haidt, J., \& Gable, S.L. (2008). Beyond reciprocity: Gratitude and relationships in everyday life. Emotion, 8, 425-429.

American Psychiatric Association. (2000). Diagnostic and Statistical Manual, 4th Edition, Text Revision. Washington, DC: Author.

Andrews, G., \& Wilkinson, D.D. (2002). The prevention of mental disorders in young people. Medical Journal of Australia, 177, 97-100.

Anthony, E. J. (1974). The syndrome of the psychologically invulnerable child. In E. J. Antho-ny, \& C. Koupernik (Eds.), The child in his family: Children at psychiatric risk (pp. 529-545). New York: Wiley.

Anthony, E.J. and Cohler, B.J. (eds) (1987). The Invulnerable Child. New York: Guilford Press. 
Asakawa, K. (2004). Flow experience and autotelic personality in Japanese college students: How do they experience challenges in daily life? Journal of Happiness Studies, 5, 123154. doi:10.1023/ B:JOHS.0000035915.97836.89.

Asakawa, K. (2009). Flow Experience, Culture, and Well-being: How Do Autotelic Japanese College Students Feel, Behave, and Think in Their Daily Lives? Journal of Happiness Studies, 11(2), 205-223. doi:10.1007/s10902-008-9132-3

Ashby, F. G., Isen, A. M., \& Turken, A. U. (1999). A neuropsychological theory of positive affect and its influence on cognition. Psychological Review, 106(3), 529-50.

Australian Institute for Primary Care (2005). Evaluations of MindMatters Plus \& MindMatters Plus GP Final Report. Retrieved October, 2010, from http://www.mindmatters.edu.au-/verve/_resources/mmplusgp_final.pdf

Baldwin, C. P., \& Baldwin, A. L. (1970). Children's Judgments of Kindness. Child Development, 41(1), 29. doi:10.2307/1127387

Bargh, J. A., \&. Chartrand, T. L. (1999). The unbearable automaticity of being. American Psychologist 54, 462-479.

Baron, R. A. (1984) . Reducing organizational conflict- an incompatible response approach. Journal of Applied Psychology, 69, 272-279.

Barrett, P. M., \& Pahl, K. M. (2006). School-based intervention: examining a universal approach to anxiety management. Australian Journal of Guidance and Counselling, 16, $55-75$

Barrett, P. M., \& Turner, C. M. (2004). Prevention strategies. In T. L. Morris, \& J. S. March (Eds.), Anxiety Disorders in Children and Adolescents (2nd ed.). (pp. 371-386) New York: The Guilford Press.

Barry, C. M., \& Wentzel, K. R. (2006). Friend influence on prosocial behavior: The role of motivational factors and friendship characteristics. Developmental Psychology, 42, 153163.

Baumeister, R. F., \& Leary, M. R. (1995). The need to belong: desire for interpersonal attachments as a fundamental human motivation. Psychological Bulletin, 117(3), 497-529.

Bayer, J. K., \& Sanson, A. V. (2003). Preventing the Development of Emotional Mental Advances in the Field Preventing the Development of Emotional Mental Health Problems from Early Childhood : Recent Advances in the Field. International Journal of Mental Health Promotion, 5(3), 4-16. 
Beeghly, M., \& Cicchetti, D., (1994). Child maltreatment, attachment and the self system: Emergence of an internal state lexicon in toddlers at high social risk. Development and Psychopathology, 6, 5-30.

Behnke, A. O., Piercy, K. W., \& Diversi, M. (2004). Educational and occupational aspirations of Latino youth and their parents. Hispanic Journal of Behavioral Sciences, $26,16-35$.

Bertocci, P. A., \& Millard, R. M. (1963). Personality and the good: Psychological and Ethical Perspectives. New York: McKay.

Bieling, P. J., McCabe, R. E., \& Antony, M. M. (2006). Cognitive-behavioral therapy in groups. Michigan: Guilford Press.

Biswas-Diener, R. (2006). From the equator to the North Pole: A study of character strengths. Journal of Happiness Studies, 7, 293-310.

Blechman, E. A., \& Culhane, S. E. (1993). Aggressive, depressive, and prosocial coping with affective challenges in early adolescence. Special Issue: Affective expression and emotion in early adolescence. Journal of Early Adolescence, 13, 361-382.

Black, S., Pulford, J., Christie, G., \& Wheeler, A. (2010). Differences in New Zealand Secondary School Students' reported Strengths and Difficulties. New Zealand Journal of Psychology, 39(3), 19-24.

Block, J., \& Kremen, A. M. (1996). IQ and ego-resiliency: conceptual and empirical connections and separateness. Journal of Personality and Social Psychology, 70(2), 349-61.

Bijttebier, P., Raes, F., Vasey, M. W., \& Feldman, G. C. (2012). Responses to positive affect predict mood symptoms in children under conditions of stress: a prospective study. Journal of Abnormal Child Psychology, 40(3), 381-9. doi:10.1007/s10802-011-9579-2

Blum, R. W. (1998). Healthy youth development as a model for youth health promotion: review. Journal of Adolescent Health, 22, 368-375.

Boehm, J. K., \& Lyubomirsky, S. (2009). The promise of sustainable happiness. In S. J. Lopez \& C. R. Snyder (Eds.), Oxford Handbook of Positive Psychology (2 ed., pp. 667677). New York, NY: Oxford University Press.

Boehm, J. K., Lyubomirsky, S., \& Sheldon, K. M. (2008). Spicing up kindness: The role of variety in the effects of practicing kindness on improvements in mood, happiness, and self-evaluations. Manuscript in preparation.

Bono, G., \& Froh, J.J. (2009). Gratitude in school: Benefits to students and schools. In R. Gilman, E.S. Huebner, \& M. Furlong (Eds.), Handbook of positive psychology in schools (pp. 77-88). New York: Routledge. 
Boulton, M. J., \& Smith, P. K. (1992). The social nature of play fighting and play chasing: Mechanisms and strategies underlying cooperation and compromise. In J. H. Barkow, L. Cosmides, \& J. Tooby (Eds.), The adapted mind: Evolutionary psychology and the generation of culture (pp. 429-444). New York: Oxford University Press.

Bradburn, N. M. (1969). The structure of psychological well-being (Vol. 318).

Bromley, E., Johnson, J.G., \& Cohen, P. (2006). Personality strengths in adolescence and decreased risk of developing mental health problems in early adulthood. Comprehensive Psychiatry, 47, 315-324.

Bryan, T., Mathur, S., \& Sullivan, K. (1996). The impact of positive mood on learning. Learn-ing Disabilities Quarterly, 19, 153-162.

Bryant, F. B. (1989). A four-factor model of perceived control: Avoiding, coping, obtaining, and savoring. Journal of Personality, 57, 773-797.

Bryant, F. B., Chadwick, E. D., \& Kluwe, K. (2011). Understanding the Processes that Regulate Positive Emotional Experience: Unsolved Problems and Future Directions for Theory and Research on Savoring. International Journal of Wellbeing, 1(1), 107-126. doi:10.5502/ijw.v1i1.18

Bryant, F. B., \& Veroff, J. (2007). Savoring: A new model of positive experience. Mahwah, NJ: Lawrence Erlbaum.

Bryce, J., \& Haworth, J. (2002). Wellbeing and flow in sample of male and female office workers. Leisure Studies, 21, 249-263. doi:10.1080/0261436021000030687.

Burns, R. A., \& Anstey, K. J. (2010). The Connor-Davidson Resilience Scale (CD-RISC): Testing the invariance of a uni-dimensional resilience measure that is independent of positive and negative affect. Personality and Individual Differences, 48(5), 527-531. doi:10.1016/j.paid.2009.11.026

Bynner, J., Joshi, H., \& Tsatsas, M. (2000). Obstacles and Opportunities on the Route to Adulthood: Evidence From Rural and Urban Britain. London: Smith Institute.

Cacioppo, J. T., \& Gardner, W. L. (1999). Emotion. Annual Review of Psychology, 50, 191214.

Cafasso, L. L., Bryant, F. B., \& Jose, P. E. (1994). A scale for measuring children's savoring beliefs. Paper presented at the 102nd annual American Psychological Association convention.

Calear, A. L., \& Christensen, H. (2010). Systematic review of school-based prevention and early intervention programs for depression. Journal of Adolescence, 33(3), 429-38. doi:10.1016-/j.adolescence.2009.07.004 
Caliendo, M., and S. Kopeinig. 2005. Some practical guidance for the implementation of propensity score matching. IZA Discussion Paper No. 1588. Bonn, Germany. Retrieved June, 2010, from http://ftp.iza.org/dp1588.pdf.

Cantor, N. (1990). From thought to behavior: "Having" and "doing'" in the study of personality and cognition. American Psychologist, 45, 735 - 750.

Cantor, N., \& Sanderson, C. A. (1999). Life task participation and well-being: The importance of taking part in daily life. In D. Kahneman, E. Diener, \& N. Schwartz (Eds.), Well-being: The foundation of hedonic psychology (pp. 230-243). New York: Sage.

Cardemil, E. V, Reivich, K. J., Beevers, C. G., Seligman, M. E. P., \& James, J. (2007). The prevention of depressive symptoms in low-income, minority children: two-year followup. Behaviour Research and Therapy, 45(2), 313-27. doi:10.1016/j.brat.2006.03.010

Carli, M., Delle Fave, A., \& Massimini, F. (1988). The quality of experience in the flow channels: Comparison of Italian and US students. In M. Csikszentmihalyi \& I. S. Csikszentmihalyi (Eds.), Optimal experience: Psychological studies of flow in consciousness (pp. 266-306). New York: Cambridge University Press.

Carroll, A. (2002). At-risk and not at-risk adolescent girls in single-sex and mixed-sex school settings: An examination of their goals and reputations. Westminster Studies in Education, 25, 147-162.

Carver, C. S. (2003). Pleasure as a sign you can attend to something else: Placing positive feelings within a general model of affect. Cognition and Emotion, 17, 241-261.

Carver, C. S., \& Johnson, S. L. (2009). Tendencies Toward Mania and Tendencies Toward Depression Have Distinct Motivational, Affective, and Cognitive Correlates. Cognitive Therapy and Research, 33(6), 552-569. doi:10.1007/s10608-008-9213-y

Cederblad, S. (1996). Fifty years of epidemiologic studies in child and adolescent psychiatry in Sweden. Nordic Journal of Psychiatry, 50, 55-66.

Chadwick, E. D. (2012). The Structure of Adolescent and Adult Savoring and its Relationship to Feeling Good and Functioning Well. Unpublished doctoral dissertation. Victoria University of Wellington, New Zealand.

Challen, A. (2009). UK Resilience Programme evaluation. Interim report. London, UK: London School of Economics.

Cheng, S. T., \& Chan, A. C. (2005). Measuring psychological well-being in the Chinese. Personality and Individual Differences, 38, 1307-1316. 
Chen, X., Rubin, K. H., \& Li, B. (1995). Depressed mood in Chinese children: Relations with school performance and family environment. Journal of Consulting and Clinical Psychology, 63, 938-947.

Cheung, M. (2006). Therapeutic Games and Guided Imagery: Tools for Mental Health and School Professionals Working with Children, Adolescents, and their families. Chicago: Lyceum Books.

Cicchetti, D., \& Toth, S. L. (1998). The development of depression in children and adolescents. American Psychologist, 53, 221-241.

Clark, L. A., \& Watson, D. (1991). Tripartite model of anxiety and depression: Psychometric evidence and taxonomic implications. Journal of Abnormal Psychology, 100, 316-336.

Clarke, S., \& Haworth, J. (1994). 'Flow' experience in the daily lives of sixth-form college students. The British Journal of Psychology, 85, 511-523.

Cohen, P., \& Cohen, J. (2001). Life values and mental health in adolescence. In P. Schmuck \& K. M. Sheldon (Eds.), Life goals and well-being. Gottingen: Hogrefe and Huber Publishers.

Cohn, M. A., Fredrickson, B. L., Brown, S. L., Mikels, J. a, \& Conway, A. M. (2009). Happiness unpacked: positive emotions increase life satisfaction by building resilience. Emotion (Washington, D.C.), 9(3), 361-8. doi:10.1037/a0015952

Cosmides, L., \& Tooby, J. (2000). Evolutionary psychology and the emotions. In M. Lewis \& J. M. H. Jones (Eds.), Handbook of Emotions (pp. 91-115). New York: Guilford Press.

Constantine, N., \& Benard, B. (2001). California Healthy Kids Survey Resilience Assessment Module Technical Report. Public Health.

Constantine, N. A., Benard, B., \& Diaz, M. (1999). Measuring Protective Factors and Resilience Traits in Youth: The Healthy Kids Resilience Assessment. Paper presented at the Seventh Annual Meeting of the Society for Prevention Research. New Orleans, LA.

Corno, L., \& Mandinach, E. B. (1983). The role of cognitive engagement in classroom learning and motivation. Educational Psychologist, 18, 88-108

Cottle, T. J., \& Klineberg, S. L. (1974). The present of things past. New York: MacMillan.

Cowen, E. L., Wyman, P. A., Work, W. C., Kim, J. Y., Fagen, D. B., \& Magnus, K. B. (1997). Follow-up study of young stress-affected \& stress-resilient urban children. Development and Psychopathology, 9, 564-577.

Craske, M. G. (2010). Cognitive-Behavioral Therapy. Washington, DC: American Psycholog-ical Association. 
Csikszentmihalyi, M. (1982). Toward a psychology of optimal experience. In L. Wheeler (Ed.), Review of personality and social psychology (Vol. 2, pp. 13-36). Beverly Hills: Sage.

Csikszentmihalyi, M. (1988). The flow experience and human psychology. In M.

Csikszentmihalyi \& I. Csikszentmihalyi (Eds.), Optimal experience: Psychological studies of flow in consciousness (pp. 15-35). Cambridge, England: Cambridge University Press.

Csikszentmihalyi, M. (1990). Flow: The psychology of optimal experience. New York: Harper Collins.

Csikszentmihalyi, M. (1993). The evolving self. New York: Harper Collins.

Csikszentmihalyi, M. (1997). Finding flow. New York: Basic Books.

Csikszentmihalyi, M. (1999). If we are so rich, why aren't we happy? American Psychologist, 54(10), 821-827. doi:10.1037//0003-066X.54.10.821

Csikszentmihalyi, M, \& Rathunde, K. (1998). The development of the person: An experiential perspective on the ontogenesis of psychological complexity. In R. M. Lerner (Ed.), Handbook of child psychology (5th ed., Vol. 1). New York: Wiley.

Csikszentmihalyi, M., Rathunde, K., \& Whalen, S. (1993). Talented teenagers: The roots of success \& failure. New York: Cambridge University Press.

Dahlin, L., \& Cederblad, S. (1993). Protective factors for individuals brought up in a highrisk environment with regard to the risk for a psychiatric or social disorder. Nordic Journal of Psychiatry, 45, 53-9.

Damásio, B. F., \& Borsa, J. C. (2011). 14-Item Resilience Scale (RS-14): Psychometric Properties of the Brazilian Version. Nursing, 19(3), 131-146.

Davidson, R. J., (1988). EEG measures of cerebral asymmetry: conceptual and methodological issues. The International Journal of Neuroscience 39, 71-89.

Davidson, R. J. (1992). Anterior Cerebral Asymmetry and the Nature of Emotion. Brain and Cognition, 20, 125-151.

Davidson, R. J. (2000). Affective style, psychopathology, and resilience: Brain mechanisms and plasticity. American Psychologist, 55, 1196-1214.

Dehejia, R. H. \& Wahba, S. (2002), Propensity Score Matching Methods for Nonexperimental Causal Studies. The Review of Economics and Statistics, 84(1), 151161. Data available at: http://www.nber.org/\%7Erdehejia/nswdata.html. 
Delle Fave, A., \& Massimini, F. (1992). The ESM and the measurement of clinical change: A case of anxiety syndrome. In M. W. de Vries (Ed.), The experience of psychopathology (pp. 280-289). Cambridge, England: Cambridge University Press.

deMan, A. F., \& Leduc, C. P. (1995). Suicidal ideation in high school students: Depression and other correlates. Journal of Clinical Psychology, 51, 173-181.

Dempster, A. P., Laird, N. M., \& Rubin, D. B. (1977). Maximum likelihood estimation from incomplete data via the EM algorithm (with discussion). Journal of the Royal Statistical Society, Series B, 39, 1-38.

DeShea, L. (2003). A scenario-based scale of willingness to forgive. Individual Differences Research, 1, 201-217.

Derryberry, D., \& Tucker, D.M. (1994). Motivating the focus of attention. In P.M.

Neidenthal \& S. Kitayama (Eds.), The heart's eye: Emotional influences in perception and attention (pp. 167-196). San Diego: Academic Press.

Diener, E., \& Emmons, R. A. (1984). The independence of positive and negative affect . Journal of Personality and Social Psychology, 47(5), 1105-1117.

Diener, E., Emmons, R. A., Larsen, R. J., \& Griffin, S. (1985). The Satisfaction With Life Scale. Journal of Personality Assessment, 49(1), 71-5. doi:10.1207/s15327752jpa4901_13

Diener, Ed, \& Biswas-Diener, R. (2008). Happiness: Unlocking the Mysteries of Psychological Wealth. Malden: Blackwell Publishing.

Diener, E., Scollon, C. N., \& Lucas, R. E. (2009). The evolving concept of subjective wellbeing: The multifaceted nature of happiness. Social Indicators Research Series, 39, $67-$ 100.

Diener, E., Suh, E. M., Lucas, R. E., \& Smith, H. L. (1999). Subjective well-being: Three decades of progress. Psychological Bulletin, 125, 276-302.

Duckworth, A. L., Steen, T. a, \& Seligman, M. E. P. (2005). Positive psychology in clinical practice. Annual Review of Clinical Psychology, 1, 629-51.

doi:10.1146/annurev.clinpsy.1.102803.144154

Dumont, M., \& Provost, M. A. (1999). Resilience in Adolescents : Protective Role of Social Support, Coping Strategies, Self-Esteem, and Social Activities on Experience of Stress and Depression. Journal of Youth and Adolescence, 28(3), 343-363.

Duncan, G.D. \& Brooks-Gunn, J. (Eds) (1997). Consequences of Growing Up Poor. New York: Russell Sage Foundation. 
Dunn, R., Griggs, S. A., Olson, J., Beasley, M., Gorman, B. S., \& Taylor, P. (1995). MetaAnalytic and Validation of the Dunnand Dunn Model of learning-Style Preferences. Journal of Educational Research, 88(6), 353-362.

Eisenberg, N. (1986). Altruistic emotion, cognition and behavior. Hillsdale, NJ: Erlbaum. Eisenberg, N., Zhou, Q., \& Koller, S. (2001). Brazilian adolescents’ prosocial moral judgment and behavior: Relations to sympathy, perspective taking, gender-role orientation, and demographic characteristics. Child Development, 72, 518-534. doi:10.1111/1467-8624.00294.

Eisenberger, R., Jones, J. R., Stinglhamber, F., Shanock, L., \& Randall, A. T. (2005). Flow experiences at work: For high need achievers alone? Journal of Organizational Behavior, 26, 755-775. doi:10.1002/ job.337.

Emmons, R. A., \& McCullough, M. E. (2003). Counting blessings versus burdens: An experimental investigation of gratitude and subjective well-being in daily life. Journal of Personality and Social Psychology, 84(2), 377-389. doi:10.1037/0022-3514.84.2.377

Epel, E S., McEwen, B. S., \& Ickovics, J. R. (1998). Embodying psychological thriving: Physical thriving in response to stress. Journal of Social Issues, 54, 301-322.

Essen, J., \& Wedge, P. (1978). Continuities in Childhood Disadvantage. London: Heineman. Farwell, L., \& Wohlwend-Lloyd, R. (1998). Narcissistic processes: Optimistic expectations, favorable self-evaluations, and self-enhancing attributions. Journal of Personality, 66, $65-83$.

Fanous, A. H., Prescott, C., \& Kendler, K. S. (2004). The prediction of thoughts of death or self-harm in a population-based sample of female twins. Psychological medicine, 34(2), 301-12.

Feng, X., Keenan, K., Hipwell, A. E., Henneberger, A. K., Rischall, M. S., Butch, J., Coyne, C., Boeldt, D., Hinze, A. K., \& Babinski, D. E. (2009). Longitudinal associations between emotion regulation and depression in preadolescent girls: moderation by the caregiving environment. Developmental Psychology, 45(3), 798-808. doi: $10.1037 / \mathrm{a} 0014617$

Folkman, S., \& Moskowitz, J. T. (2000). Positive affect and the other side of coping. American Psychologist, 55(6), 647-654. doi:10.1037//0003-066X.55.6.647

Folkman, S., \& Moskowitz, J. T. (2004). Coping: Pitfalls and promise. Annual Review of Psychology, 55, 745-774.

Fredrickson, B. L. (1998). What good are positive emotions? Review of General Psychology: Special Issue: New Directions in Research on Emotion, 2, 300-319. 
Fredrickson, B. L. (2001). The role of positive emotions in positive psychology: The broaden-and-build theory of positive emotions. American Psychologist: Special Issue, 56, 218-226.

Fredrickson, B. L., \& Cohn, M. A. (2008). Positive emotions. In M. Lewis, J. Haviland,\&L. F. Barrett (Eds.), Handbook of emotions (3rd ed.). New York: Guilford Press.

Fredrickson, B. L., \& Levenson, R. W. (1998). Positive emotions speed recovery from the cardiovascular sequelae of negative emotions. Cognition and Emotion, 12, 191-220.

Fredrickson, B. L., \& Losada, M. F. (2005). Positive affect and the complex dynamics of human flourishing. American Psychologist, 60(7), 678-86. doi:10.1037/0003066X.60.7.678

Fredrickson, B. L., Mancuso, R. A., Branigan, C., \& Tugade, M. M. (2000). The undoing effect of positive emotions. Motivation and Emotion, 24, 237-258.

Fredrickson, B. L., Tugade, M. M., Waugh, C. E., \& Larkin, G. R. (2003). What good are positive emotions in crisis? A prospective study of resilience and emotions following the terrorist attacks on the United States on September 11th, 2001. Journal of Personality and Social Psychology, 84(2), 365-376. doi:10.1037/0022-3514.84.2.365

Frijda, N. H. (1988). The laws of emotion. American Psychologist, 43, 349-358.

Froh, J., Bono, G., \& Emmons, R. (2010). Being Grateful is Beyond Good Manners: Gratitude and Motivation to Contribute to Society Among Early Adolescents. Motivation and Emotion, 34, 144-157.

Froh, J.J., Kashdan, T. B., Ozimkowski, K. M., \& Miller, N. (2009). Who benefits the most from a gratitude intervention in children and adolescents? Examining positive affect as a moderator. The Journal of Positive Psychology, 4(5), 408-422. doi:10.1080/17439760902992464

Froh, J. J., Kashdan, T. B., Yurkewicz, C., Fan, J., Allen, J., \& Glowacki, J., (2010). The benefits of passion and absorption in activities: Engaged living in adolescents and its role in psychological well-being. The Journal of Positive Psychology, 5(4), 311-332. doi:10.1080/17439760.2010.498624.

Froh, J.J., Sefick, W. J., \& Emmons, R. A. (2008). Counting blessings in early adolescents: an experimental study of gratitude and subjective well-being. Journal of School Psychology, 46(2), 213-233. doi:10.1016/j.jsp.2007.03.005

Gable, S. L., Reis, H. T., Impett, E. A., \& Asher, E. R. (2004). What do you do when things go right? The intrapersonal and interpersonal benefits of sharing positive events. Journal of Personality and Social Psychology, 87, 228-245. 
Gander, F., Proyer, R. T., Ruch, W., \& Wyss, T. (2012). Strength-Based Positive Interventions: Further Evidence for Their Potential in Enhancing Well-Being and Alleviating Depression. Journal of Happiness Studies. doi:10.1007/s10902-012-9380-0

Garmezy, N. (1971). Vulnerability research and the issue of primary prevention. American Journal of Orthopsychiatry, 41(1), 101-116.

Garmezy, N. (1991). Resilience in children's adaptation to negative life events and stressed environments. Pediatric Annals, 20, 459-466.

Garmezy, N., Masten, A. S., \& Tellegen, A. (1984). The Study of Stress and Competence in Children: A Building Block for Developmental Psychopathology. Child Development, $55,97-111$.

Gentzler, A. L., Morey, J. N., Palmer, C. A., \& Yi, C. Y. (2012). Young Adolescents' Responses to Positive Events: Associations With Positive Affect and Adjustment. The Journal of Early Adolescence. doi:10.1177/0272431612462629

Geraghty, A. W. A., Wood, A. M., \& Hyland, M. E. (2010). Dissociating the facets of hope: Agency and pathways predict dropout from unguided self-help therapy in opposite directions. Journal of Research in Personality, 44(1), 155-158. doi:10.1016/j.jrp.2009.12.003

Gillham, J.E. (2003). Targeted prevention is not enough. Commentary on "Treatment is not enough: We must prevent major depression in women." Prevention and Treatment, 6.

Gillham, J., Adams-Deutsch, Z., Werner, J., Reivich, K., Coulter-Heindl, V., Linkins, M., Winder, B., Winder, B., Peterson, C., Park, N., Abenavoli, R., Contero, A., \& Seligman, M. E. P. (2011). Character strengths predict subjective well-being during adolescence. The Journal of Positive Psychology, 6(1), 31-44. doi:10.1080/17439760.2010.536773

Gillham, J. E., Hamilton, J., Freres, D. R., Patton, K., \& Gallop, R. (2006). Preventing depression among early adolescents in the primary care setting: A randomized controlled study of the Penn Resiliency Program. Journal of Abnormal Child Psychology, 34, 203-219.

Gillham, J. E., Reivich, K. J., Freres, D. R., Chaplin, T. M., Shatté, A. J., Samuels, B., Elkon, A. G. L., Litzinger, S., Lascher, M., Gallop, R., \& Seligman, M. E. P. (2007). Schoolbased prevention of depressive symptoms: A randomized controlled study of the effectiveness and specificity of the Penn Resiliency Program. Journal of Consulting and Clinical Psychology, 75(1), 9-19. doi:10.1037/0022-006X.75.1.9 
Ginsburg, G. S., \& Drake, K. L. (2002). School-based treatment for anxious AfricanAmerican adolescents: a controlled pilot study. Journal of the American Academy of Child and Adolescent Psychiatry, 41, 768-775.

Gladstone, T. R. G., \& Kaslow, N. J. (1995). Depression and attributions in children and adolescents: A meta-analytic review. Journal of Abnormal Child Psychology, 23, 597606.

Goldberg, D. P. (1972). The detection of psychiatric illness by questionnaire: A technique for the identification and assessment of non-psychotic psychiatric illness. London: Oxford University Press.

Goodman, R. (2001) Psychometric properties of the Strengths and Difficulties Questionnaire (SDQ). Journal of the American Academy of Child and Adolescent Psychiatry, 40, 1337 1345.

Gordon Rouse, K. A. (2001). Resilient students' goals and motivation. Journal of Adolescence, 24, 461-472

Gotlib, I. H., Lewinsohn, P. M., \& Seeley, J. R. (1995). Symptoms versus a diagnosis of depression: differences in psychosocial functioning. Journal of Consulting and Clinical Psychology, 63, 90-100.

Govindji, R., \& Linley, P. A. (2007). Strengths use, self-concordance and well-being: Implication for strengths coaching and coaching psychologist. International Coaching Psychology Review, 2, 143-153.

Graham, S., \& Barker, G. P. (1990). The down side of help: An attributional-developmental analysis of helping behavior as low-ability cue. Journal of Educational Psychology, 82, $7-14$.

Gratz, K. L., \& Roemer, L. (2004). Multidimensional assessment of emotion regulation and dysregulation: Development, factor structure, and initial validation of the difficulties in emotion regulation scale. Journal of Psychopathology and Behavioral Assessment, 26, $41-54$.

Green, L. S., Oades, L. G., \& Grant, A. M. (2006). Cognitive-behavioral, solution-focused life coaching: Enhancing goal striving, well- being, and hope. Journal of Positive Psychology, 1, 142-149.

Greenberg, M. T., Domitrovich, C., \& Bumbarger, B. (2001). The prevention of mental disorders in school-aged children: Current state of the field. Prevention \& Treatment, 4(1), 1-62. doi:10.1037//1522-3736.4.1.41a 
Gross, J. J., Richards, J. M., \& John, O. P. (2006). Emotion regulation in everyday life. In D. K. Snyder, J. A. Simpson, \& J. N. Hughes (Eds.), Emotion regulation in families: Pathways to dysfunction and health (pp. 13-35). Washington, DC: American Psychological Association.

Grossman, P., Niemann, L., Schmidt, S., \& Walach, H. (2004). Mindfulness-based stress reduction and health benefits. Journal of Psychosomatic Research, 57, 35-43.

Han, S. (1988). The relationship between life satisfaction and flow in elderly Korean immigrants. In M. Csikszentmihalyi \& I. S. Csikszentmihalyi (Eds.), Optimal experience: Psychological studies of flow in consciousness (pp. 138-149). New York, NY: Cambridge University Press.

Hart, D., \& Carlo, G. (2005). Moral development in adolescence. Journal of Research on Adolescence, 15, 223-233. doi:10.1111/j.1532-7795.2005.00094.x.123

Hasui, C., Igarashi, H., Shikai, N., Shono, M., Nagata, T., \& Kitamura, T. (2009). The Resilience Scale : A Duplication Study in Japan. Journal of Personality and Social Psychology, 2, 15-22.

Heine, C. (1996). Flow and achievement in mathematics. Unpublished doctoral dissertation, University of Chicago.

Hektner, J. (1996). Exploring optimal personality development: A longitudinal study of adolescents. Unpublished doctoral dissertation, University of Chicago.

Hidi, S. (1990). Interest and its contribution as a mental resource for learning. Review of Educational Research, 60, 549-571.

Hill, J. L., Waldfogel, J., Brooks-Gunn, J., \& Han, W. J. (2005). Maternal employment and child development: A fresh look using newer methods. Developmental Psychology, 41, 833- 850. doi:10.1037/0012-1649.41.6.833

Hjemdal, O., Friborg, O., Stiles, T. C., Martinussen, M., \& Rosenvinge, J. H. (2006). A New Scale for Adolescent Resilience: Grasping the Central Protective Resources behind Healthy development. Measurement and Evaluation in Counseling and Development, $39,84-96$.

Hodges \& Blythe (1992) Improving service delivery to High-Risk Families: Home-based practice. Families in Society, 73, 259-65.

Hofer, J., \& Chasiotis, A. (2003). Congruence of life goals and implicit motives as predictors of life satisfaction: Cross-cultural implications of a study of Zambian male adolescents. Motivation and Emotion, 27, 251-271. 
Horowitz, J. L., Garber, J., Ciesla, J. A., Young, J. F., \& Mufson, L. (2007). Prevention of depressive symptoms in adolescents: a randomized trial of cognitive- behavioral and interpersonal prevention programs. Journal of Consulting and Clinical Psychology, 75, 693-706.

Howing, P., Wodarski, J., Kurtz, P. D., \& Gaudin, J. M., (1990). The empirical base for the implementation of Social Skills Training with Maltreated Children. Social Work, 35, 460-467

Hughes, G. J. (2001). Aristotle on Ethics. London: Routledge.

Hunter, J. P., \& Csikszentmihalyi, M. (2003). The Positive Psychology of Interested Adolescents. Journal of Youth and Adolescence, 32(1), 27-35. doi:10.1023/A:1021028306392

Huppert, F. A., \& Whittington, J. E. (2003). Evidence for the independence of positive and negative well-being : Implications for quality of life assessment. British Journal of Health Psychology, 8(1), 107-122.

Hurley, D. B., \& Kwon, P. (2012). Results of a Study to Increase Savoring the Moment: Differential Impact on Positive and Negative Outcomes. Journal of Happiness Studies, 13(4), 579-588.

Huta, V., \& Hawley, L. (2008). Psychological Strengths and Cognitive Vulnerabilities: Are They Two Ends of the Same Continuum or Do They Have Independent Relationships with Well-being and Ill-being? Journal of Happiness Studies, 11(1), 71-93. doi:10.1007/s10902-008-9123-4

Hyman, S. E., Malenka, R. C., \& Nestler, E. J. (2006). Neural mechanisms of addiction: the role of reward-related learning and memory. Annual Review of Neuroscience, 29, 56598. doi:10.1146/annurev.neuro.29.051605.113009

Inghilleri, P. (1999). From subjective experience to cultural evolution. New York: Cambridge University Press.

Ingram, R. E. (2006). Blending the Good With the Bad : Integrating Positive Psychology and Cognitive Psychotherapy. Journal of Cognitive Psychotherapy, 20(2), 117-122.

Ingrid, B., Majda, R., \& Dubravka, M. (2009). Life Goals and Well-Being: Are Extrinsic Aspirations Always Detrimental to Well-Being ? Psychological Topics, 18(2), 317-334.

Isen, A. M. (1987). Positive affect, cognitive processes, and social behavior. Advances in Experimental Social Psychology, 20, 203-253.

Isen, A. M. \& Diamond, G. A. (1989). Affect and automaticity, in J.S. Uleman and J.A. Bargh (Eds.), Unintended Thought (pp. 124-152). New York: Guilford Press. 
Isen, A. M., Daubman, K. A., \& Nowicki, G. P. (1987). Positive affect facilitates creative problem solving. Journal of Personality and Social Psychology, 52, 1122-1131.

Isen, A. M., \& Geva, N. (1987). The influence of positive affect on acceptable level of risk: The person with a large canoe has a large worry. Organizational Behavior and Human Decision Processes, 39, 145-154.

Isen, A. M., Johnson, M. S., Mertz, E., \& Robinson, G. F. (1985). The influence of positive affect on the unusualness of word associations. Journal of Personality and Social Psychology, 48, 1413-1426.

Ishimura, I., \& Kodama, M. (2006). Dimensions of flow experience in Japanese college students: Relation between flow experience and mental health. Journal of Health Psychology, 13, 23-34.

Jacelon, C. S. (1997). The trait and process of resilience. Journal of Advanced Nursing, 25, $123-129$.

Janoff-Bulman, R., \& Berger, A. R. (2000). The other side of trauma: Towards a psychology of appreciation. In J. H. Harvey \& E. D. Miller (Eds.), Loss and trauma: General and close relationship perspectives (pp. 29-44). Philadelphia: Brunner-Routledge.

John, O. P., \& Gross, J. J. (2004). Healthy and unhealthy emotion regulation: Personality processes, individual differences, and life span development. Journal of Personality, 72, 6, 1301-1333.

Jose, P.E., \& Huntsinger, C.S. (2005). Moderation and mediation effects of coping by Chinese American and European American adolescents. The Journal of Genetic Psychology, 166, 16- 44.

Jose, P. E., Lim, B. T., \& Bryant, F. B. (2012). Does savoring increase happiness ? A daily diary study. The Journal of Positive Psychology, 7(3), 176-187.

Kabat-Zinn, J. (1990). Full Catastrophe Living: Using the Wisdom of Your Body and Mind to Face Stress, Pain, and Illness. New York: Delta.

Kahn, B. E., \& Isen, A. M. (1993). The influence of positive affect on variety-seeking among safe, enjoyable products. Journal of Consumer Research, 20, 257-270.

Kasser, T. (2002). Sketches for a self-determination theory of values. In E. L. Deci \& R. M. Ryan (Eds.), Handbook of self-determination research (pp. 123-140). Rochester, NY: University of Rochester Press.

Kasser, T., \& Ryan, R. M. (1993). A dark side of the American dream: Correlates of financial success as a central life aspiration. Journal of Personality and Social Psychology 65, 410-422. 
Kasser, T., \& Ryan, R. M. (1996). Further Examining the American Dream: Differential Correlates of Intrinsic and Extrinsic Goals. Personality and Social Psychology Bulletin, 22(3), 280-287. doi:10.1177/0146167296223006

Kohlberg, L. (1976). Moral stages and moralization: The cognitive developmental approach. In T. Lickona (Ed.), Moral Development and Behavior (pp. 31-35). New York: Holt, Rinehart, \& Winston.

Kohlberg, L. (1981). The philosophy of moral development: Moral stages and the idea of justice. San Francisco, CA: Harper and Row.

Kovacs M. (1985). The Children's Depression Inventory (CDI). Psychopharmacol Bulletin, $21,995-1124$.

Kovacs, M. (2008). Children's depression inventory: Technical manual. Toronto: MultiHealth Systems.

Kowalenko, N., Rapee, R.M., Simmons, J., Wignall, A., Hoge, R., Whitefield, K., Starling, J., Stonehouse, R., \& Baillie, A.J. (2005). Short-term effectiveness of a school-based early intervention program for adolescent depression. Clinical Child Psychology and Psychiatry, 10, 4, 493-507.

Kubovy, M. (1999). On the pleasures of the mind. In D. Kahneman, E. Diener, \& N. Schwartz (Eds.), Well-being: The foundations of hedonic psychology (pp. 134-154). New York: Russell Sage Foundation.

Lamborn, S. D., Fischer, K. W., \& Pipp, S. (1994). Constructive criticism and social lies: A developmental sequence for understanding honesty and kindness in social interactions. Developmental Psychology, 30(4), 495-508. doi:10.1037//0012-1649.30.4.495

Langston, C. A. (1994). Capitalizing on and coping with daily-life events: Expressive responses to positive events. Journal of Personality and Social Psychology, 67, 1112 1125 .

Larsen, R. J., \& Prizmic, Z. (2004). Affect regulation. In R. F. Baumeister \& K. D. Vohs, (Eds.), Handbook of Self-regulation. Research, Theory, and Application. New York: Guilford Press.

Layous, K., Chancellor, J., Lyubomirsky, S., Wang, L., \& Doraiswamy, P. M. (2011). Delivering happiness: Translating positive psychology intervention research for treating major and minor depressive disorders. Journal of Alternative and Complementary Medicine, 17(8), 675-83. doi:10.1089/acm.2011.0139

Lazarus, R. S. (1993). From psychological stress to the emotions: A history of changing outlooks. Annual Review of Psychology, 44, 1-21. 
LeDoux, J. E. (1995). Emotion : Clues from the brain. Annual Review of Psychology, 46, $209-235$.

Lekes, N., Gingras, I., Philippe, F. L., Koestner, R., \& Fang, J. (2009). Parental autonomysupport, intrinsic life goals, and well-being among adolescents in China and North America. Journal of Youth and Adolescence, 39(8), 858-69. doi:10.1007/s10964-0099451-7

Lewinsohn, P.M., Clarke, G.N., Hops, H., \& Andrews, J. (1990). Cognitive-behavioral treatment for depressed adolescents. Behavior Therapy, 21, 385-401.

Lightfoot, M., Stein, J. A, Tevendale, H., \& Preston, K. (2011). Protective factors associated with fewer multiple problem behaviors among homeless/runaway youth. Journal of Clinical Child and Adolescent Psychology, 40(6), 878-89. doi:10.1080/15374416.2011.614581

Linley, P. A. (2008). Average to A?: Realising strengths in yourself and others. Coventry: CAPP Press.

Linley, P. A., Joseph, S., Harrington, S., \& Wood, A. M. (2006). Positive Psychology: Past, present, and (possible) future. The Journal of Positive Psychology, 1(1), 3-16. doi:10.1080/17439760500372796

Linley, P. A., Maltby, J., Wood, A., Joseph, S., Harrington, S., Peterson, C., Park, N., \& Seligman, M. E. P. (2007). Character strengths in the United Kingdom: The VIA inventory of strengths. Personality and Individual Differences, 43, 341-351.

Linley, P. A., Nielsen, K. M., Wood, A. M., Gillett, R., \& Biswas-Diener, R., (2010). Using signature strengths in pursuit of goals: Effects on goal progress, need satisfaction, and well-being, and implications for coaching psychologists. International Coaching Psychology Review, 5(1), 8-17.

Lowry-Webster, H. M., Barrett, P. M., \& Dadds, M. R. (2001). A Universal Prevention Trial of Anxiety and Depressive Symptomatology in Childhood: Preliminary Data from an Australian Study. Behaviour Change, 18(1), 36-50. doi:10.1375/bech.18.1.36

Luthar, S. S. (1999). Poverty and children's adjustment. Thousand Oaks, CA: Sage.

Lyubomirsky, S. (2001). Why are some people happier than others? The role of cognitive and motivational processes in well-being. American Psychologist, 56, 239-249.

Lyubomirsky, S (2007). The How of Happiness. A scientific approach to getting the life you want. New York: Penguin Group.

Lyubomirsky, S., King, L., \& Diener, E. (2005). The benefits of frequent positive affect: Does happiness lead to success? Psychological Bulletin,131, 803-855. 
Lyubomirsky, S., \& Lepper, H. S. (1999). A measure of subjective happiness: preliminary reliability and construct validation. Social Indicators Research, 137-155.

Lyubomirsky, S., \& Tucker, K. (1998). Implication of individual differences in subjective happiness for perceiving, interpreting, and thinking about life events. Motivation and Emotion, 22, 155-186.

Lyubomirsky, S., Sheldon, K. M., \& Schkade, D. (2005). Pursuing Happiness: The Architecture of Sustainable Change. Review of General Psychology, 9(2), 111-131. doi:10.1037/1089-2680.9.2.111

Maginness, A. (2007). The development of resilience - A Model. Unpublished doctoral dissertation. University of Canterbury, New Zealand.

Marjoribanks, K. (1993). Perceived parents'support for learning and aspirations for Australian adolescents. Perceptual and Motor Skills, 77, 840-842

Marjoribanks, K. (2003). Learning environments, family contexts, educational aspirations and attainment: A moderation- mediation model extended. Learning Environments Research, 6, 247-265.

Martin, P. R., Reece, J., Lauder, S., \& McClelland, A. (2011). A Randomised Controlled Trial of a Social Support Intervention. Applied Psychology: Health and Well-Being, 3(1), 44-65. doi:10.1111/j.1758-0854.2010.01044.x

Martos, T. \& Kopp, M. S. (2012). Life goals and well-being: Does financial status matter? Evidence from a representative Hungarian sample. Social Indicator Research. 105,(3), 561-568.

Masia-Warner, C., Nangle, D. W., \& Hansen, D. J. (2006). Bringing evidence-based child mental health services to the schools: general issues and specific populations. Education and Treatment of Children, 29, 165-172.

Massimini, F., \& Carli, M. (1988). The systematic assessment of flow in daily experience. In M. Csikszentmihalyi \& I. Csikszentmihalyi (Eds.), Optimal experience: Psychological studies of flow in consciousness (pp. 266-287). New York: Cambridge University Press.

Massimini, F., Csikszentmihalyi, M., \& Carli, M. (1987). The monitoring of optimal experience: A tool for psychiatric rehabilitation. Journal of Nervous and Mental Disease, 175, 545-549.

Massimini, F., \& Delle Fave, A. (2000). Individual development in a bio-cultural perspective. American Psychologist, 55, 24-33.

Masten, A. S. (2001). Ordinary magic: Resilience processes in development. American Psychologist, 56(3), 227-238. doi:10.1037//0003-066X.56.3.227 
Masten, A., Best, K., \& Garmezy, N. (1990). Resilience and development: Contributions from the study of children who overcome adversity. Development and Psychopathology, 2, 425-444

Masten, A. S., Burt, K. B., Roisman, G. I., Obradović, J., Long, J. D., \& Tellegen, A. (2004). Resources and resilience in the transition to adulthood: continuity and change. Development and Psychopathology, 16(4), 1071-94.

Masten, A. S., Garmezy, N., Tellegen, A., Pellegrini, D.S., Larkin, K., \& Larsen, A. (1988). Competence and stress in school children: The moderating effects of individual and family qualities. Journal for Child Psychiatry and Psychology, 28, 745-764.

McCullough, M. E., Emmons, R. A., \& Tsang, J.A. (2002). The grateful disposition: A conceptual and empirical topography. Journal of Personality and Social Psychology, 82, $112-127$.

McEwen, B. S. (1998). Protective and damaging effects of stress mediators. New England Journal of Medicine, 338, 171-179.

McGorry, P. D., Purcell, R., Hickie, I. B., \& Jorm, A. F. (2007). Investing in youth mental health is a best buy. Medical Journal of Australia, 187, S5-S7.

McNulty, J. K., \& Fincham, F. D. (2012). Beyond positive psychology? Toward a contextual view of psychological processes and well-being. The American Psychologist, 67(2), 101-10. doi:10.1037/a0024572

Meehan, M., Durlak, J., \& Bryant, F.B. (1993). The relationship of social support to positive life events and subjective mental health in adolescents. Journal of Community Psychology, 21, 49-55.

Merry, S., McDowell, H., Wild, C. J., Bir, J., \& Cunliffe, R. (2004). A randomized placebocontrolled trial of a school-based depression prevention program. Journal of the American Academy of Child and Adolescent Psychiatry, 43, 538-547.

Mezulis, A., Hyde, J .S., \& Abramson, L. Y. (2006). The developmental origins of cognitive vulnerability to depression: temperament, parenting, and negative life events. Developmental Psychology, 42, 1012-1025.

Miller, W.R., \& Rollnick, S. (2002). Motivational interviewing: Preparing people for change. New York: The Guilford Press.

Moneta, G. B. and Csikszentmihalyi, M. (1996). The effect of perceived challenges and skills on the quality of subjective experience. Journal of Personality, 64(2), 275-309.

Montessori, M. (1967). The absorbent mind. New York: Holt Rinehart and Winston. 
Moran, P. B., \& Eckenrode, J. (1992). Protective personality characteristics among adolescent victims of maltreatment. Child Abuse and Neglect, 16, 743-754.

Moulds, M.L., Kandris, E., Starr, S., \& Wong, A.C.M. (2007). The relationship between rumination, avoidance and depression in a non-clinical sample. Behaviour Research and Therapy, 45, 251-261.

Mrazek, P. J., \& Haggerty, R. J. (1994). Reducing risks for mental disorders: Frontiers for preventive intervention research.Washington: National Academy Press.

Nakamura, J. (1988). Optimal experience and the uses of talent. In M. Csikszentmihalyi \& I. S. Csikszentmihalyi (Eds.), Optimal experience: Psychological studies of flow in consciousness (pp. 319- 326). New York: Cambridge University Press.

Nakamura, J., \& Csikszentmihalyi, M. (2002a). The concept of flow. In C. R. Snyder \& S. J. Lopez (Eds.), Handbook of positive psychology (pp. 89-105). New York: Oxford University Press.

Nakamura, J., \& Csikszentmihalyi, M. (2002b). The construction of meaning through vital engagement. In C. Keyes \& J. Haidt (Eds.), Flourishing: Positive psychology and the life well-lived (pp. 83-104). DC: APA Books.

Nakamura, J., \& Csikszentmihalyi, M. (2008). Flow theory and research. In C. R. Snyder \& S. J. Lopez (Eds.), Handbook of positive psychology (2nd ed.). New York: Oxford University Press (in press).

Nederhof, A. J. (1985). Methods of coping with social desirability bias: A review. European Journal of Social Psychology, 15,(3), 263-280.

Neill, J. T., \& Dias, K. L. (2001). Adventure education and resilience: The double-edged sword. Journal of Adventure Education \& Outdoor Learning, 1(2), 35-42. doi:10.1080/14729670185200061

Niemiec, C. P., Ryan, R. M., \& Deci, E. L. (2009). The path taken: Consequences of attaining intrinsic and extrinsic aspirations in post-college life. Journal of Research in Personality, 43, 291- 306.

Nisbett, R. E., \& Wilson, T. D. (1977). Telling more than we can know: Verbal reports on mental processes. Psychological Review, 84, 231-259.

Noelle-Neumann, E. (1995, Spring). Allensbach Archives, AWA.

Nolen-Hoeksema, S. (1991). Responses to depression and their effects on the duration of depressive episodes. Journal of Abnormal Psychology, 100, 569-582. 
Norrish, J., \& Vella-Brodrick, D. (2009). Positive psychology and adolescents: Where are we now? Where to from here? Australian Psychologist, 44(4), 270-278. doi:10.1080/00050060902914103

Nurmi, J. E. (1994). The development of future-orientation in a life-span context. In Z. Zaleski (Ed.), Psychology of future orientation (pp. 20-616). Lublin, Poland: Wydawnictwo Towarzystwa Naukowego Katolickiego Uniwersytetu Lubelskiego.

Nurmi, J.-E., \& Pulliainen, H. (1991). The changing parent-child relationship, self-esteem, and intelligence as determinants of orientation to the future during early adolescence. Journal of Adolescence, 14, 35-51.

O’Dougherty-Wright, M., Masten, A. S., Northwood, A., \& Hubbard, J. J. (1997). Long-term effects of massive trauma: Developmental and psychobiological perspectives. In D. Cicchetti \& S. L. Toth (Eds.), Rochester Symposium on Developmental Psychopathology: Vol. 8. Developmental perspectives on trauma (pp. 181-225). Rochester, NY: University of Rochester Press.

Offord, D. R., Kraemer, H. C., Kazdin, A. E., Jensen, P. S., \& Harrington, R. (1998). Lowering the burden of suffering from child psychiatric disorder: Trade-offs among clinical, targeted, and universal interventions. Journal of the American Academy of Child and Adolescent Psychiatry, 37, 686-694.

Olsson, C. a, Bond, L., Burns, J. M., Vella-Brodrick, D. a, \& Sawyer, S. M. (2003). Adolescent resilience: a concept analysis. Journal of adolescence, 26(1), 1-11.

Ong, A. D., Bergeman, C. S., Bisconti, T. L., \& Wallace, K. A. (2006). Psychological Resilience, Positive Emotions, and Successful Adaptation to Stress in Later Life. Journal of Personality and Social Psychology, 91(4), 730-749. doi:10.1037/00223514.91.4.730

Otake, K., Shimai, S., Tanaka-Matsumi, J., Otsui, K., \& Fredrickson, B. L. (2006). Happy People Become Happier Through Kindness: a Counting Kindnesses Intervention. Journal of Happiness Studies, 7(3), 361-375. doi:10.1007/s10902-005-3650-z

Oxford Dictionaries (2012). In Pearsall, J. (Ed.) Oxford Dictionary (Online). Oxford: Oxford University Press. Retrieved July 29, 2012, from http://oxforddictionaries.com.

Park, N., \& Peterson, C. (2006a). Moral competence and character strengths among adolescents: the development and validation of the Values in Action Inventory of Strengths for Youth. Journal of Adolescence, 29(6), 891-909. doi:10.1016/j.adolescence.2006.04.011

Park, N., \& Peterson, C. (2006b). Character strengths and happiness among young children: 
Content analysis of parental descriptions. Journal of Happiness Studies, 7, 323-341.

Park, N., \& Peterson, C. (2009). Strengths of character in schools. In R. Gilman, E. S.

Huebner, \& M. J. Furlong (Eds.), Handbook of positive psychology in schools (pp. 65-

76). New York: Routledge.

Park, N., Peterson, C., \& Seligman, M. E. P. (2004). Strengths of character and well-being. Journal of Social and Clinical Psychology, 23, 603-619.

Pavot, W., \& Diener, E. (2008). The Satisfaction With Life Scale and the Emerging Construct of Life Satisfaction. The Journal of Positive Psychology, 3(2), 137-152. doi:10.1080/17439760701756946

Penick, N. I., \& Jepsen, D. A. (1992). Family functioning and adolescent career development. The Career Development Quarterly, 40, 208-222.

Peterson, C., Park, N., \& Seligman, M. E. P. (2005). Orientations to happiness and life satisfaction: The full life versus the empty life. Journal of Happiness Studies, 6, 25-41.

Peterson, C., \& Seligman, M. E. P. (2004). Character strengths and virtues: A handbook and classification. Oxford: Oxford University Press.

Piaget, J. (1965). The moral judgment of the child. New York: Free Press.

Phelps, C. D. (2001). A clue to the paradox of happiness. Journal of Economic Behavior \& Organization, 45(3), 293-300. doi:10.1016/S0167-2681(01)00147-0

Phinney, J. S., Baumann, K., \& Blanton, S. (2001). Life goals and attributions for expected outcomes among adolescents from five ethnic groups. Hispanic Journal of Behavioral Sciences, 23, 363-377.

Prince-Embury, S. (2008). The Resiliency Scales for Children and Adolescents, Psychological Symptoms, and Clinical Status in Adolescents. Canadian Journal of School Psychology, 23(1), 41-56. doi:10.1177/0829573508316592

Proctor, C., Maltby, J., \& Linley, A. P. (2011). Strengths use as a predictor of well-being and health related quality of life. Journal of Happiness Studies 10, 583-630. doi:10.1007/s10902-009-9181-2.

Quayle, D., Dziurawiec, S., Roberts, C., Kane, R., \& Ebsworthy, G. (2001). The Effect of an Optimism and Lifeskills Program on Depressive Symptoms in Preadolescence. Behaviour Change, 18(4), 194-203. doi:10.1375/bech.18.4.194

Quoidbach, J., Berry, E. V., Hansenne, M., \& Mikolajczak, M. (2010). Positive emotion regulation and well-being: Comparing the impact of eight savoring and dampening strategies. Personality and Individual Differences, 49(5), 368-373. doi:10.1016/j.paid.2010.03.048 
Raibley, J. R. (2012). Happiness is not Well-Being. Journal of Happiness Studies, 13(6), 1105-1129 doi:10.1007/s10902-011-9309-Z

Rashid, T. (2009). Positive Interventions in Clinical Practice. Journal of Clinical Psychology, 65(5), 461-466. doi:10.1002/jclp

Rashid, T., \& Anjum, A. (2008). Positive psychotherapy for young adults and children. In J. R. Z. Abela \& B. L. Hankin (Eds.), Handbook of depression in children and adolescents (pp. 250-287). New York: Guilford Press.

Rashid, T., \& Ostermann, R. F. (2009). Strength-Based Assessment in Clinical Practice. Online, 65(5), 488-498. doi:10.1002/jclp

Rathunde, K. (1988). Optimal experience and the family context. In M. Csikszentmihalyi \& I. S. Csikszentmihalyi (Eds.), Optimal experience: Psychological studies of flow in consciousness (pp. 342-363). New York: Cambridge University Press.

Reis, H. T., Smith, S. M., Carmichael, C. L., Caprariello, P. A., Tsai, F.F., Rodrigues, A., \& Maniaci, M. R. (2010). Are you happy for me? How sharing positive events with others provides personal and interpersonal benefits. Journal of Personality and Social Psychology, 99(2), 311-29. doi:10.1037/a0018344

Reivich, K., Gillham, J. E., Chaplin, T. M., \& Seligman, M. E. P. (2005). From helplessness to optimism: the role of resilience in treating and preventing depression in youth. In $\mathrm{S}$. Goldstein, \& R. B. Brooks (Eds.), Handbook of Resilience in Children (pp. 223-237). New York: Kluwer Academic/Plenum Publishers.

Richardson, G. (2002). The metatheory of resilience and resiliency. Journal of Clinical Psychology, 58(3), 307-321.

Richters, J. E., \& Martinez, P. E. (1993). Violent communities, family choices, and children's chances: An algorithm for improving the odds. Development and Psychopathology, 5, $609-627$.

Riggs, P. D., Baker, S., Mikulich, S. K., \& Young, S. E. (1995). Depression in substancedependent delinquents. Journal of the American Academy of Child and Adolescent Psychiatry, 34, 764-771.

Robins, L., \& Rutter M. (1990). Straight and Devious Pathways from Childhood to Adulthood. Cambridge: Cambridge University Press.

Robinson, E., \& Curry, J. (2005). Promoting altruism in the classroom. Childhood Education, $82(2), 68-73$.

Rottenberg, J., \& Gross, J. J. (2003). When emotion goes wrong: Realizing the promise of affective science. Clinical Psychology: Science and Practice, 10(2), 227-232. 
Roy, A., Sarchiapone, M., \& Carli, V. (2007). Low resilience in suicide attempters. Archives of suicide research : official journal of the International Academy for Suicide Research, 11(3), 265-9. doi:10.1080/13811110701403916

Ruini, C., Belaise, C., Brombin, C., Caffo, E., \& Fava, G. A. (2006). Well-being therapy in school settings: a pilot study. Psychotherapy and Psychosomatics, 75(6), 331-6. doi:10.1159/000095438

Russell, J. A., \& Carroll, J. M. (1999). On the bipolarity of positive and negative affect. Psychological Bulletin, 125(1), 3-30.

Rutter, M. (1979). Protective factors in children's responses to stress and disadvantage. In M. W. Kent \& J. E. Rolf (Eds.), Primary prevention in psychopathology: Social competence in children (Vol. 8, pp. 49-74). Hanover, NH: University Press of New England.

Rutter, M. (1987). Psychosocial resilience and protective mechanisms. American Journal of Orthopsychiatry, 57, 316-331.

Rutter, M. (1989). Pathways from childhood to adult life. Journal of Child Psychology and Psychiatry, 30, 25-51.

Rutter, M. (1993). Resiilience: Some conceptual considerations. Journal of Adolescent Health, 14(8), 626-631.

Rutter, M. (1999). Resilience concepts and findings: implications for family therapy. Journal of Family Therapy, 21(2), 119-144. doi:10.1111/1467-6427.00108

Rutter, M., \& Madge, M. (1976). Cycles of Disadvantage: A Review of Research. London: Heinemann Educational Books.

Ryan, R.M., Chirkov, V.I., Little, T.D., Sheldon, K.M., Timoshina, E., \& Deci, E.L. (1999). The American dream in Russia: Extrinsic aspirations and well-being in two cultures. Personality and Social Psychology Bulletin, 25, 1509-1524.

Ryff, C. D., \& Keyes, C. L. (1995). The structure of psychological well-being revisited. Journal of Personality and Social Psychology, 69(4), 719-27.

Ryff, C. D., \& Singer, B. H. (2006). Know Thyself and Become What You Are: A Eudaimonic Approach to Psychological Well-Being. Journal of Happiness Studies, 9(1), 13-39. doi:10.1007/s10902-006-9019-0

Sandvik, E., Diener, E., \& Seidlitz, L. (2009). Subjective well-being: The convergence and stability of self-report and non-self-report measures. Social Indicators Research Series, 39, 119-137.

Savage, J. A. (2011). Increasing Adolescents' Subjective Well-Being : Effects of a Positive Psychology Intervention in Comparison to the Effects of Therapeutic Alliance, Youth 
Factors, and Expectancy for Change. Unpublished doctoral dissertation. Open access by the USF Graduate School at Scholar Commons. University of South Florida, America. http://scholarcommons.usf.edu/etd/3333.

Sawatzky, R., Gadermann, A., \& Pesut, B. (2009). An investigation of the relationships between spirituality, health status and quality of life in adolescents. Applied Research in Quality of Life, 4(1), 5-22. doi: 10.1007/s11482-009-9065-y.

Schimmack, U., Oishi, S., Furr, R. M., \& Funder, D. C. (2004). Personality and life satisfaction: A facet-level analysis. Personality and Social Psychology Bulletin, 30, $1062-1075$.

Schinke, S.P., Schilling, R.F., \& Snow, W. H., (1987). Stress Management with Adolescents at the Junior High School Transition: An outcome evaluation of Coping Skills. Journal of Human Stress, 13, 16-22.

Schmidt, J. (2003). Correlates of reduced misconduct among adolescents facing adversity. Journal of Youth and Adolescence, 32, 439-452.

Schoon,I., Bynner, J., Joshi, H., Parsons, S., Wiggins, R.D., \& Sacker, A. (2002) The influence of context, timing and duration of risk experiences for the passage from childhood to early adulthood. Child Development, 73, 1486-1504.

Seaton, E. K., Scottham, K. M., \& Sellers, R. M. (2006). The status model of racial identity development in African American adolescents: evidence of structure, trajectories, and well-being. Child development, 77(5), 1416-26. doi:10.1111/j.1467-8624.2006.00944.x

Seligman, M. E. (1990). Learned optimism. New York: Alfred A. Knopf.

Seligman, M. E. P. (2002). Authentic happiness: Using the new positive psychology to realize your potential for lasting fulfilment. New York, NY: Free Press.

Seligman, M., \& Csikszentmihalyi, M. (2000). Positive psychology: An introduction. American Psychologist, 55, 5-14.

Seligman, M. E. P., Ernst, R. M., Gillham, J., Reivich, K., \& Linkins, M. (2009). Positive education: positive psychology and classroom interventions. Oxford Review of Education, 35(3), 293-311. doi:10.1080/03054980902934563

Seligman, M. E. P., Rashid, T., \& Parks, A. C. (2006). Positive psychotherapy. The American psychologist, 61(8), 774-88. doi:10.1037/0003-066X.61.8.774

Seligman, M. E. P., Steen, T. A., Park, N., \& Peterson, C. (2005). Positive psychology progress: Empirical validation of interventions. American Psychologist, 60, 410-421.

Schiefele, U., Krapp, A., \& Winteler, A. (1992). Interest as a predictor of academic achievement: A meta-analysis of research. In K. A. Renninger, S. Hidi, \& A. Krapp 
(Eds.), The role of interest in learning and development (pp. 183-212). Hillsdale, NJ: Erlbaum.

Sheldon, K. M., Abad, N., Ferguson, Y., Gunz, A., Houser-Marko, L., Nichols, C. P., \& Lyubomirsky, S. (2009). Persistent pursuit of need-satisfying goals leads to increased happiness: A 6-month experimental longitudinal study. Motivation and Emotion, 34(1), 39-48. doi:10.1007/s11031-009-9153-1

Sheldon, K. M., \& Elliot, A. J. (1998). Not all personal goals are personal: Comparing autonomous and controlled reasons as predictors of effort and attainment. Personality and Social Psychology Bulletin, 24, 546-557.

Sheldon, K.M. \& Kasser, T. (1995). Coherence and congruence: Two aspects of personality integration. Journal of Personality and Social Psychology 68, 531-543.

Sheldon, K. M., Kasser, T., Smith, K., \& Share, T. (2002). Personal Goals and Psychological Growth: Testing an Intervention to Enhance Goal Attainment and Personality Integration. Journal of Personality, 70(1), 5-31.

Shernoff, D. J., \& Csikszentmihalyi, M. (2006). Ch.11 Flow in schools. In the Handbook of Positive Psychology in Schools. (R. Gilman, E. S. Huebner, \& M. J. Furlong, Eds.) (pp. 131-146). New York: Routledge.

Shernoff, D. J., Csikszentmihalyi, M., Schneider, B., \& Shernoff, E. S. (2003). Student Engagement in High School Classrooms from the Perspective of Flow Theory. School Psychology Quarterly, 18(2), 158-176. doi:10.1521/scpq.18.2.158.21860

Shimai, S., Otake, K., Park, N., Peterson, C., \& Seligman, M. E. P. (2006). Convergence of character strengths in American and Japanese young adults. Journal of Happiness Studies, 7, 311-322.

Shochet, I. M., Dadds, M. R., Holland, D., Whitefield, K., Harnett, P. H., \& Osgarby, S. M. (2001). The efficacy of a universal school-based program to prevent adolescent depression. Journal of Clinical Child Psychology, 30(3), 303-15. doi:10.1207/S15374424JCCP3003_3

Shoshani, A., \& Slone, M. (2012). Middle School Transition from the Strengths Perspective: Young Adolescents' Character Strengths, Subjective Well-Being, and School Adjustment. Journal of Happiness Studies, 7(2). doi:10.1007/s10902-012-9374-y

Sin, N. L., \& Lyubomirsky, S. (2009). Enhancing Well-Being and Alleviating Depressive Symptoms With Positive Psychology Interventions: A Practice-Friendly Meta-Analysis. Journal of Clinical Psychology, 65(5), 467-487. doi:10.1002/jclp 
Smith, J.C. (1990). Cognitive-Behavioral Relaxation Training: A New System of Strategies for Treatment and Assessment. New York: Springer.

Smith, M. (2001). Creating Community in the Classroom. Kappa Delta Pi Record, 37(3), 111-115. doi:http://dx.doi.org/10.1080/00228958.2001.10518479

Snyder, C. R., \& Lopez, S. J. (2005). Handbook of Positive Psychology. Oxford: Oxford University Press.

Snyder, C. R., Lopez, S. J., Shorey, H. S., Rand, K. L., \& Feldman, D. B. (2003). Hope theory, measurements, and applications to school psychology. School Psychology Quarterly, 18(3), 122-139. doi: 10.1521/scpq.18.2.122.21854.

Sobell, M.B., \& Sobell, L.C. (1993). Problem drinkers: Guided self-change treatment. New York: The Guilford Press.

Solomon, R. C. (1977). The passions. Garden City, NY: Anchor Books.

Souza, M. De, \& McLean, K. (2012). Bullying and violence: Changing an act of disconnectedness into an act of kindness. Pastoral Care in Education, 30(2), 165-180. doi:http://dx.doi.org/10.1080/02643944.2012.679955

Spasovksi, O. (2009). Subjective well-being in student teachers: Relation with extrinsic and intrinsic motivation, self-esteem, competence and collectivism. Paper presented at the ECSNI-2009, November 12-14, Zadar, Croatia.

Spirito, A., Esposito-Smythers, C., Wolff, J., \& Uhl, K. (2011). Cognitive-behavioral therapy for adolescent depression and suicidality. Child and Adolescent Psychiatric Clinics of North America, 20(2), 191-204. doi:10.1016/j.chc.2011.01.012.

Steele, J. P., \& Fullagar, C. J. (2009). Facilitators and outcomes of student engagement in a college setting. The Journal of psychology, 143(1), 5-27. doi:10.3200/JRLP.143.1.5-27

Steger, M. F., Hicks, B. M., Kashdan, T. B., Krueger, R. F., \& Bouchard, T. J. (2007). Genetic and environmental influences on the positive traits of the values in action classification, and biometric covariance with normal personality. Journal of Research in Personality, 41, 524-539.

Stipek, D., Recchia, S., \& McClintic, S. (1992). Self evaluation in young children. Monographs of the Society for Research in Child Development, 57 (1, Serial No. 226)

Teigen, K. H. (1997). Luck, envy, and gratitude: It could have been different. Scandinavian Journal of Psychology, 38, 313-323.

Tennen, H., \& Affleck, G. (2002). Benefit-finding and benefit-reminding. In C.R. Synder and S.J. Lopez (Eds), Handbook of Positive Psychology. NY: Oxford University Press. 
The Act of Kindness (2012). Retrieved September 27, 2012, from http://www.theactofkindness.org.au/home

Thompson, R. A. (1994). Emotion regulation: A theme in search of definition. In N. A. Fox (Ed.), The development of emotion regulation: Biological and behavioral considerations. Monographs of the Society for Research in Child Development, 59(2-3, Serial No. 240), 25-52.

Toepfer, S. M., Cichy, K., \& Peters, P. (2011). Letters of Gratitude: Further Evidence for Author Benefits. Journal of Happiness Studies, 13(1), 187-201. doi:10.1007/s10902011-9257-7

Tomarken, A. J., Dichter, G. S., Garber, J., \& Simien, C. (2004). Resting frontal brain activity: linkages to maternal depression and socio-economic status among adolescents. Biological Psychology, 67(1-2), 77-102. doi:10.1016/j.biopsycho.2004.03.011

Tomarkenand, A. J., \& Anita, D. (1998). Frontal Brain Asymmetry and Depression : A Selfregulatory Perspective. Cognition and Emotion, 12(3), 387-420.

Tsang, J.A. (2006). The effects of helper intention on gratitude and indebtedness. Motivation and Emotion, 30, 199-205.

Tugade, M. M., \& Fredrickson, B. L. (2004). Resilient individuals use positive emotions to bounce back from negative emotional experiences. Journal of Personality and Social Psychology, 86(2), 320-33. doi:10.1037/0022-3514.86.2.320

Tugade, M. M., \& Fredrickson, B. L. (2007). Regulation of positive emotions: Emotion regulation strategies that promote resilience. Journal of Happiness Studies, 8, 311-334. doi:10.1007/s10902-006-9015-4

Tugade, M. M., Fredrickson, B. L., \& Barrett, L. F. (2004). Psychological resilience and positive emotional granularity: Examining the benefits of positive emotions on coping and health. Journal of Personality, 72(6), 1161-1190. doi:10.1111/j.14676494.2004.00294.x

Underwood, B., \& Moore, B. (1982). Perspective-taking and altruism. Psychological Bulletin, 91, 143-173. doi:10.1037/0033-2909.91.1.143.

Van Dierendonck, D. (2004). The construct validity of Ryff's scales of psychological wellbeing and it's extension with spiritual well-being. Personality and Individual Differences, 36, 629-643.

Veisson, M. (1999). Depression symptoms and emotional states in parents of disabled and non-disabled children. Social Behavior and Personality, 27, 87-98 
Vinson, J. A. (2002). Children with asthma: Initial development of the Child Resilience Model: Practice applications of research. Pediatric Nursing, 28, 149-158.

Wagnild, G. (2009). A Review of the Resilience Scale. Journal of Nursing Measurement, 17(2), 105-113. doi:10.1891/1061-3749.17.2.105

Wagnild, G. M., \& Young, H. M.. (1993). Development and psychometric validation of the Resilience Scale. Journal of Nursing Measurement, 1, 165-178.

Wahl, M. S., Patak, M. A., Pössel, P., \& Hautzinger, M. (2011). A school-based universal programme to prevent depression and to build up life skills. Journal of Public Health, 19(4), 349-356. doi:10.1007/s10389-011-0400-z

Wassef, A., Collins, M. L., Ingham, D., \& Mason, G. (1995). In search of effective programs to address students' emotional distress and behavioral problems. Adolescence, 30, 757-777.

Watkins, P.C., Woodward, K., Stone, T., \& Kolts, R.D. (2003). Gratitude and happiness: The development of a measure of gratitude and its relationship with subjective well-being. Social Behavior and Personality, 31, 431-452.

Watson, D. (2000). Mood and Temperament. New York: Guilford Press.

Watson, D., \& Clark, L. A. (1988). Development and Validation of Brief Measures of Positive and Negative Affect: The PANAS Scales. Journal of Personality and Social Psychology, 54(6), 1063-1070.

Watson, D., Hubbard, B., \& Wiese, D. (2000). General traits of personality and affectivity as predictors of satisfaction in intimate relationships: Evidence from self- and partnerratings. Journal of personality, 68, 413-449

Watson, J. (1895). Hedonic theories from Aristippus to Spencer. New York, NY: Macmillan.

Weikart, D. \& Schweinhart, L. (1992) High/Scope Preschool Program Outcome. In J. McCord \& R. E. Tremblay (Eds.), Interventions from Birth through Adolescence (pp. 67-86). New York: Guilford press.

Weiner,B. (1985).An attributional theory of achievement motivation and emotion. Psychological Review, 92, 548-573.

Weissman, A. N., \& Beck, A. T. (1978). Development and validation of the Dysfunctional Attitudes Scale. Paper presented at the annual meeting of the American Educational Research Association, November, Toronto, Ontario, Canada.

Wells, A. J. (1988). Self-esteem and optimal experience. In M. Csikszentmihalyi \& I. S. Csikszentmihalyi (Eds.), Optimal experience: Psychological studies of flow in consciousness (pp. 327-341). New York: Cambridge University Press. 
Wells, R. D., \& Schwebel, A. I. (1987). Chronically ill children and their mothers: Predictors of resilience and vulnerability to hospitalization and surgical stress. Journal of Developmental and Behavioral Pediatrics, 18, 83-89.

Wentzel, K. R. (1994). Relations of social goal pursuit to social acceptance, classroom behavior, and perceived social support. Journal of Educational Psychology, 86, 173182.

Wentzel, K. R., Filisetti, L., \& Looney, L. (2007). Adolescent prosocial behavior: The role of self-processes and contextual cues. Child Development, 78, 895-910

Werner, E. E. (1995). Resilience in development. Current Directions in Psychological Sciences, 4, 81-85.

Werner, E. E., Bierman, J. M., \& French, F. E. (1971). The children of Kauai. Honolulu: Hawaii, University of Hawaii Press.

Werner, E.E. and Smith, R.S. (1982). Vulnerable but Invincible: A Longitudinal Study of Resilient Children and Youth. New York: McGraw Hill.

Werner, E.E, \& Smith, R.S. (1992). Overcoming the Odds: High-Risk Children from Birth to Adulthood. New York: Cornell University Press.

Werner, E.E. \& Smith, R.S. (2002). Journey's from Childhood to Midlife: Risk, Resilience and Recovery. NY: Cornell University Press.

Wood, A. M., Froh, J. J., \& Geraghty, A. W. A. (2010). Gratitude and well-being: A review and theoretical integration. Clinical Psychology Review, 30(7), 890-905. doi:10.1016/j.cpr.20-10.03.005

Wood, A. M., Joseph, S., Lloyd, J., \& Atkins, S. (2009). Gratitude influences sleep through the mechanism of pre-sleep cognitions. Journal of Psychosomatic Research, 66, 43-48.

Wood, A. M., Joseph, S., \& Maltby, J. (2008). Gratitude uniquely predicts satisfaction with life: Incremental validity above the domains and facets of the five factor model. Personality and Individual Differences, 45, 49-54.

Wood, A. M., Maltby, J., Gillett, R., Linley, P. A., \& Joseph, S. (2008). The role of gratitude in the development of social support, stress, and depression: Two longitudinal studies. Journal of Research in Personality, 42(4), 854-871. doi:10.1016/j.jrp.2007.11.003

Wood, A. M., \& Tarrier, N. (2010). Positive Clinical Psychology: a new vision and strategy for integrated research and practice. Clinical Psychology Review, 30(7), 819-29. doi:10.1016/j.cpr.2010.06.003

Woods, B.R. (2008). Kiwi ACE: A school-based preventive depression programme. Unpublished doctoral dissertation. Deakin University, Australia. 
Woods, B., \& Jose, P. E. (2011). Effectiveness of a School-Based Indicated Early Intervention Program for Māori and Pacific Adolescents. Journal of Pacific Rim Psychology, 5(1), 40-50.

Xiao, C., Shao, X. M., Olive, M. F., Griffin, W. C., Li, K.-Y., Krnjević, K., Zhou, C., \& Ye, J. (2009). Ethanol facilitates glutamatergic transmission to dopamine neurons in the ventral tegmental area. Neuropsychopharmacology, 34(2), 307-18. doi:10.1038/npp.2008.99

Yalom, I. D. (1995). The theory and practice of group psychotherapy (4th ed.). New York: Basic Books. 


\section{Appendix A:}

Realistic Thinking Form, from Kiwi ACE Group Book

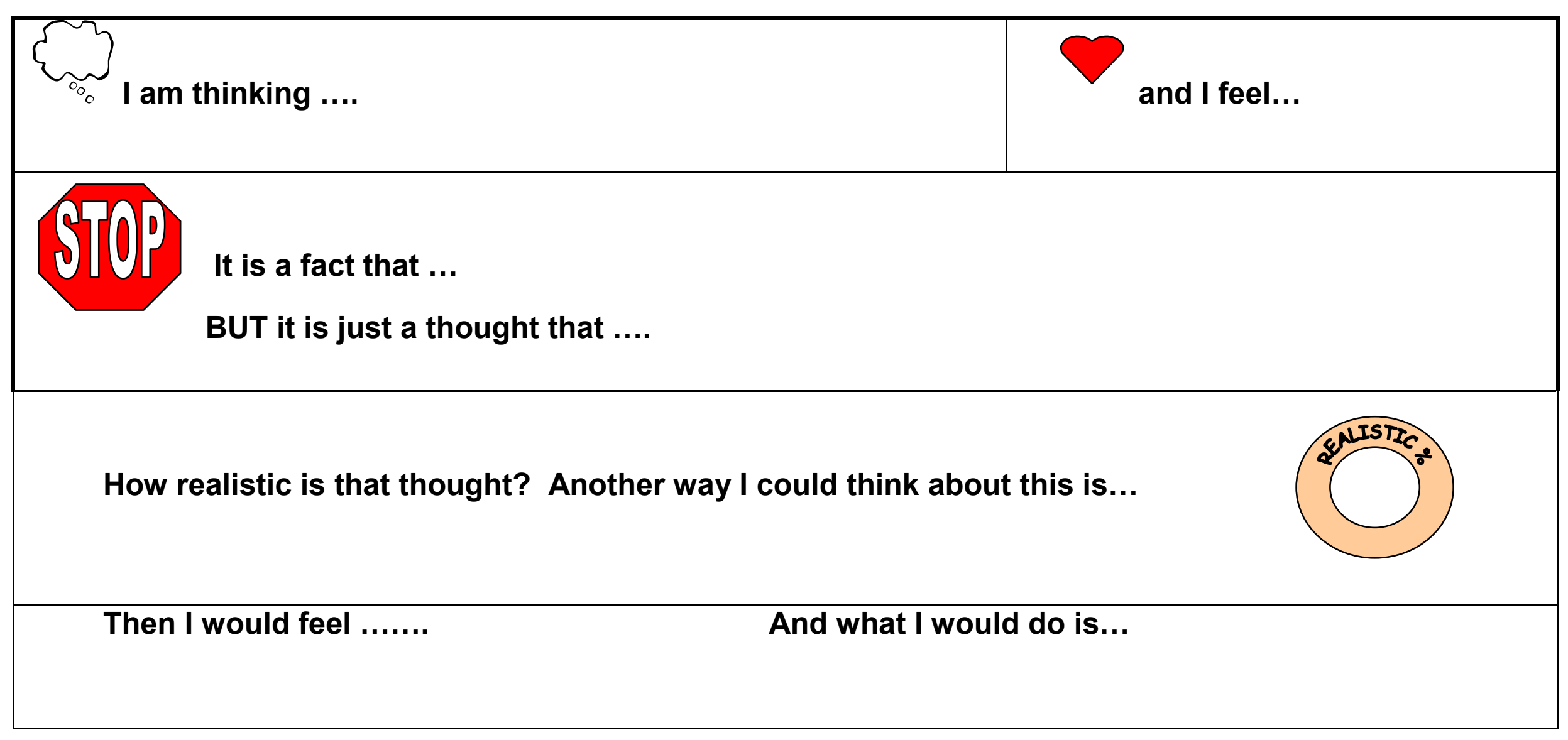




\section{Appendix B:}

Problem Solving Steps from the Kiwi ACE Facilitator's Handbook

\section{Identify the problem}

2. Brainstorm possible solutions.

- Brainstorming is thinking of every possible solution, no matter how impractical or ridiculous it sounds.

- Think of creative solutions you haven't thought of before.

3. Look at consequences of the solutions. What would happen if...?

4. Place them in order from best to worst. Which seems best to try?

5. Try out first solution and assess whether it worked.

6. If it doesn't work, try the next idea. 


\section{Appendix C:}

Problems to solve from the Kiwi ACE Facilitator's Handbook

\section{Problem Solving Examples}

1. A science assignment is due. It is a large project that is worth $30 \%$ of the year's mark. You have been putting it off and you now only have one week before the assignment is due.

2. You have a crush on a girl / boy and would like to ask them out on a date but you're afraid to ask her/him and don't know where to go.

3. It's Friday and you've run out of money on you cell phone. You don't get your allowance until next Tuesday, and so you can't top up your phone.

4. While your parents are out you invite friends over without your parents'/caregiver's permission and they accidentally break a window.

5. You are about to go out and your caregiver/mum/dad tells you that you are not allowed to wear the outfit you have on. 


\section{Appendix D:}

Fun Activities Form, from the Kiwi ACE Student Group Book

\begin{tabular}{|l|l|l|l|l|l|}
\hline What I did & $\begin{array}{l}\text { How long I } \\
\text { did it for }\end{array}$ & How I felt & $\begin{array}{l}\text { Feeling Scale } \\
\text { Before (0-10) }\end{array}$ & $\begin{array}{l}\text { Feeling Scale } \\
\text { After (0-10) }\end{array}$ \\
\hline & & & & \\
\hline
\end{tabular}




\section{Appendix E:}

Seven Steps of Negotiation, from the Kiwi ACE Facilitator's Handbook

1. Stay calm - body relaxed, low voice, calm breathing.

2. Can this problem be negotiated at this time? If the answer is 'no' then you may need to wait for a suitable time.

3. First listen to what the other person says and what they want.

Let them know you can see their point of view.

4. Then be assertive and say what you want

5. Negotiate by suggesting what you'd be willing to do if they did what you want.

6. Listen again and continue to negotiate until you are both satisfied.

7. If you can't come to an agreement, take a break and come back later. 


\section{Appendix F:}

Negotiation Form, from Kiwi ACE Student Group Book

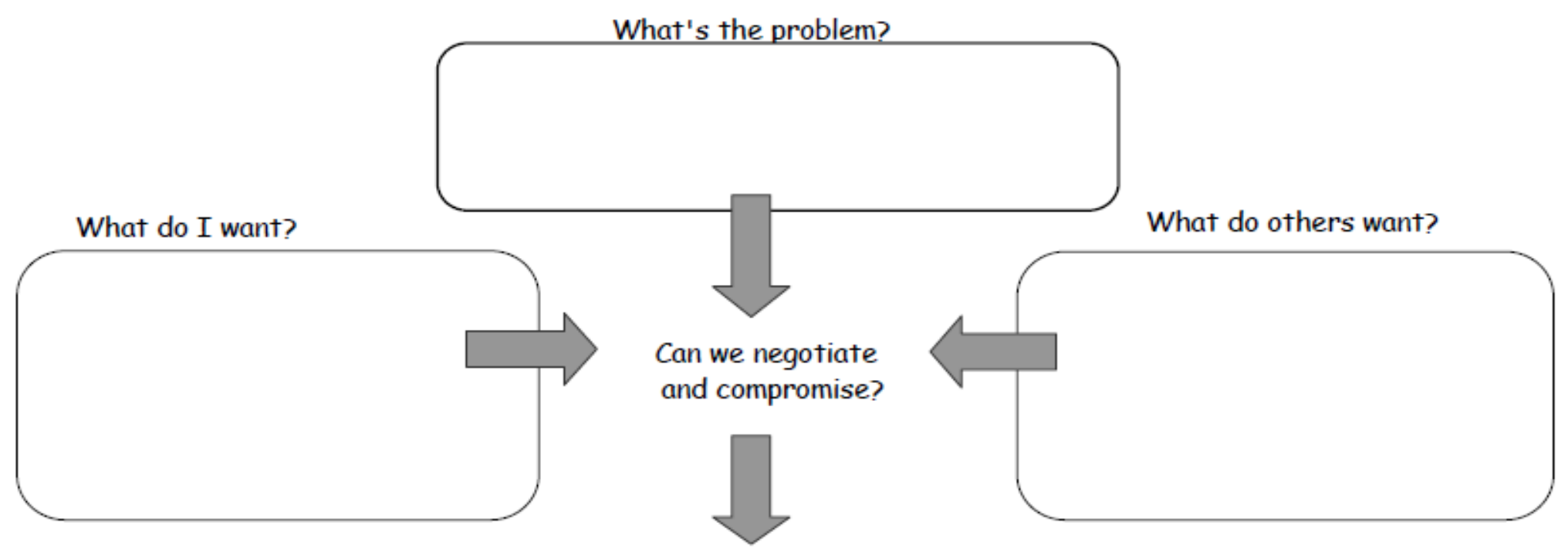

A solution with reasonable satisfaction for everyone

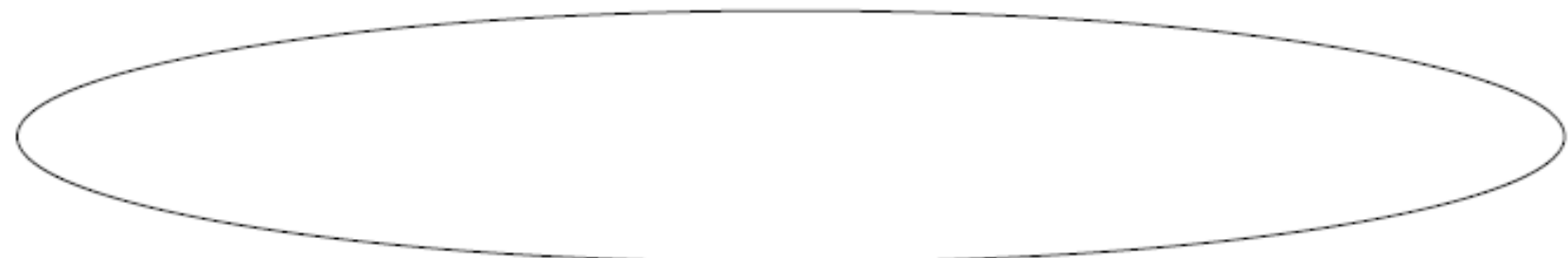




\section{Appendix G:}

Beliefs that Rob and Boost Self-esteem, from Kiwi ACE Group Book

\section{Robbers and Boosters \\ of your self-esteem}

\begin{tabular}{|c|c|}
\hline $\begin{array}{c}\text { BELIEFS that } \\
\text { ROB }\end{array}$ & BELIEFS that \\
\hline I must do everything perfectly. & $\begin{array}{r}\text { I accept that I'm not perfect } \\
\text { and I have limitations. }\end{array}$ \\
\hline $\begin{array}{c}\text { I must be successful } \\
\text { at everything. }\end{array}$ & $\begin{array}{c}\text { I can't be good at everything but I } \\
\text { am good at some things. }\end{array}$ \\
\hline $\begin{array}{c}\text { I must be approved of } \\
\text { by everyone. }\end{array}$ & $\begin{array}{r}\text { I am a worthwhile person } \\
\text { and I don't need to prove it. }\end{array}$ \\
\hline $\begin{array}{c}\text { The world should be fair } \\
\text { all the time. }\end{array}$ & It's OK to make mistakes. \\
\hline \multicolumn{2}{|c}{} \\
\hline
\end{tabular}




\section{Appendix H:}

Steps to Boost Self-esteem, from Kiwi ACE Group Book
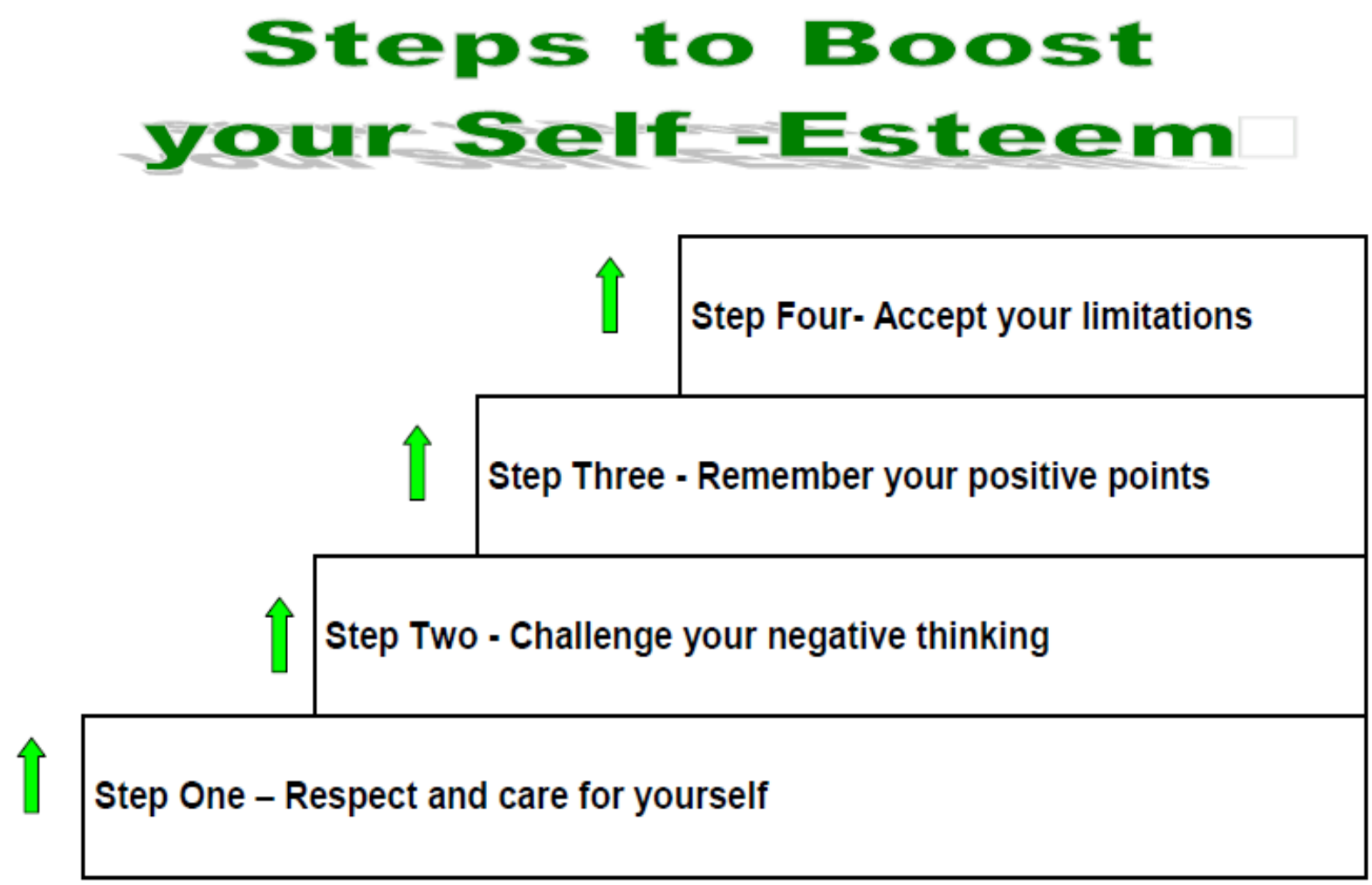


\section{Appendix I:}

Kiwi ACE Idea Sheet, from Kiwi ACE Facilitator's Handbook

Kiwi ACE ideas 1 - Link Between Thinking and Feeling

Emotions are a result of how we think and how we interpret an event.

There is more than one way to think about an event .

Extreme thinking $\quad \Rightarrow \quad \begin{aligned} & \text { Extreme Feelings } \\ & \text { anxious, down, distressed }\end{aligned}$

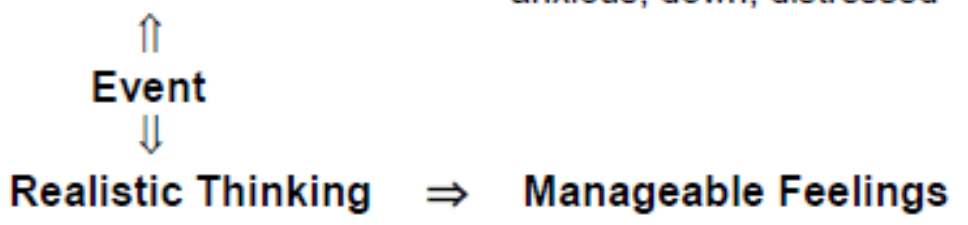

- What Realistic Thoughts about these events could change the feelings?

\begin{tabular}{|l|l|l|}
\hline Event & Thought 1 & Feeling 1 \\
\hline $\begin{array}{l}\text { Some students tell you to } \\
\text { sit somewhere else on the } \\
\text { bus. }\end{array}$ & No-one likes me & Rejected and sad \\
\cline { 2 - 3 } & Thought 2 & Feeling 2 \\
\cline { 2 - 3 } $\begin{array}{l}\text { Some students tell you to } \\
\text { sit somewhere else on the } \\
\text { bus. }\end{array}$ & & \\
\hline
\end{tabular}

\begin{tabular}{|l|l|l|}
\hline Event & Thought 1 & Feeling 1 \\
\hline $\begin{array}{l}\text { You hear your parents } \\
\text { fighting }\end{array}$ & They'll break up & Sad and worried \\
\cline { 2 - 3 } & Thought 2 & Feeling 2 \\
\cline { 2 - 3 } $\begin{array}{l}\text { You hear your parents } \\
\text { fighting }\end{array}$ & & \\
\hline
\end{tabular}




\section{Appendix I continued}

Kiwi ACE ideas 2 - Realistic Thinking (Challenging Unrealistic Thinking)

Some thoughts are unrealistic - they can be challenged.

Changing how we think can make us feel better and less down.

Be a detective and use Realistic Thinking to challenge thoughts-

A person who is usually quite friendly to you walks past without saying anything and seems to give you a dirty look.

Step 1 What am I thinking? That guy really hates me

Step 2 It's not a fact, it is just a thought Probably a belief

Step 3 Check out the evidence!

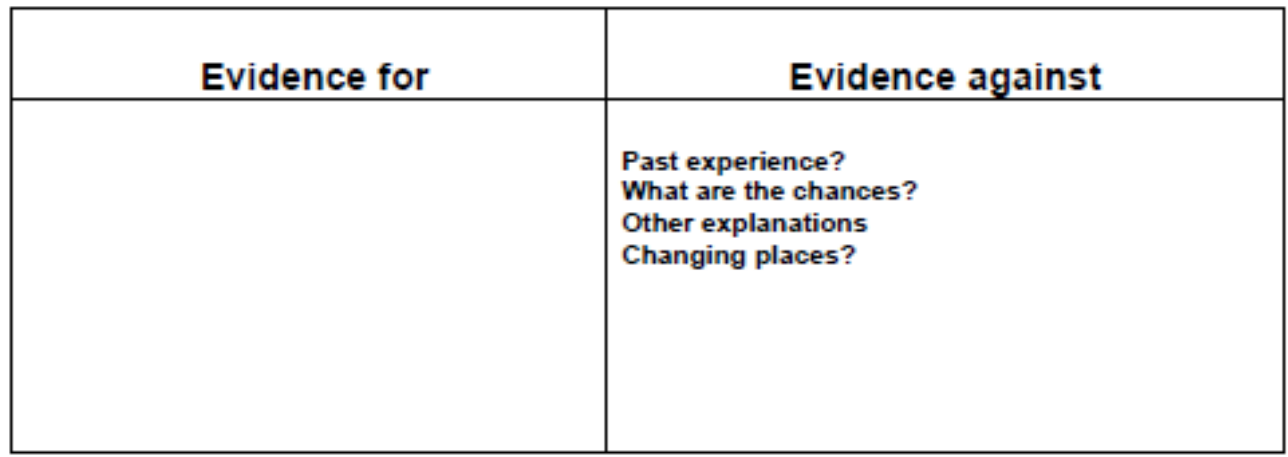

* Refer to your Realistic Thinking forms for the strategies 


\section{Appendix I continued}

Kiwi ACE ideas 3 - Assertiveness

Our behaviour with others can be aggressive, assertive or passive

There are verbal and non - verbal skills which can help us to be assertive.

Being assertive can help us get what we need and recognise the needs of others.

Being aggressive or passive means there are winners and losers.

Choose one of these events to show how a person can respond in the three different ways.

1. You are in a car with a group of friends. The driver is driving way too fast and is showing off.

2. You are with two friends who decide it would be fun to shoplift. You really do not want to be involved.

\begin{tabular}{|c|l|l|l|}
\hline & Aggressive & Assertive & Passive \\
\hline Verbal skills & & & \\
\hline $\begin{array}{c}\text { Non-Verbal } \\
\text { skills }\end{array}$ & & & \\
\hline
\end{tabular}




\section{Appendix I continued}

Kiwi ACE ideas 4 - Problem Solving

\section{Problem solving gives practical solutions for realistic difficulties.}

Sometimes realistic thinking is not enough.

We need to use Problem Solving to come up with some practical solutions if there are realistic difficulties.

Choose one of these situations or make up one of your own.

- You don't have anyone to take to the dance.

- You want to go snow boarding but you don't have any money.

- You invite some friends over when your parents are out and a window is broken.

Use the Problem Solving steps to help you decide what to do

Step 1 Identify the problem.

Step 2 Brainstorm all possible solutions.

Step 3 What would happen for each of these?

Step 4 What will work best? Put them in order.

Step 5 Give it a try. Did it work?

Step 6 If not, try the next solution. Is that better? 


\section{Appendix J:}

\section{Character Strengths, From the PAL Group Book}

\section{Character Strengths:}

\section{Appreciation of beauty and excellence}

You notice and appreciate beauty, excellence, and/or skilled performance in all areas of life, from nature to art to mathematics to science to everyday experiences.

\section{Curiosity and interest in the world}

You are curious about everything. You are always asking questions, and you find all subjects and topics fascinating. You like to explore and discover things.

\section{Bravery and moral courage}

You are a courageous person who does not shrink from threat, challenge, difficulty, or pain. You speak up for what is right even if there is opposition. You act on what you feel is right.

\section{Creativity, ingenuity and originality}

Thinking of new ways to do things is a crucial part of who you are. You are never content with doing something the normal or traditional way if a better way is possible.

\section{Self-control and discipline}

You are a disciplined person. You are usually aware of what you feel and you are in control of the things you want and your feelings.

\section{Humour and playfulness}

You like to laugh and make others laugh. Bringing smiles to the faces of other people is important to you. You try to see the lighter side of situations.

\section{Relatedness and social intelligence}

You are aware of the feelings and motives of other people. You know what to do to put others at ease. You value close relations with others.

\section{Honesty, authenticity and genuineness}

You are an honest person, not only by speaking the truth but by living your life in a genuine and authentic way. You are down to earth and do not pretend to be anything or anyone you are not.

\section{Perseverance and diligence}

You work hard to finish what you start. No matter the project, you get it done on time. You do not get distracted when you work, and you take satisfaction in completing tasks.

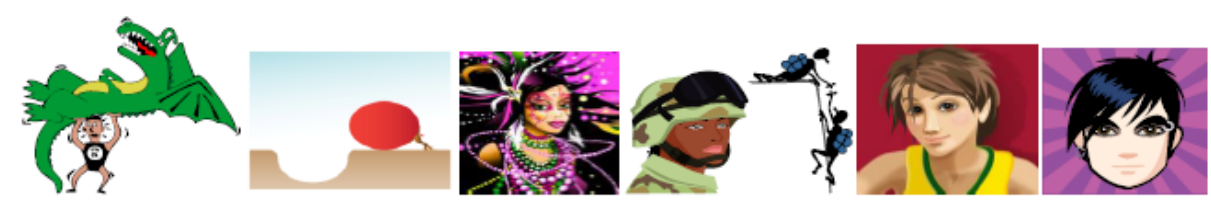




\section{Appendix K:}

\section{Character Strength Survey, from PAL Group Book}

\begin{tabular}{|c|c|c|c|c|c|c|}
\hline & $\begin{array}{c}\text { Below is a list describing different characteristics, } \\
\text { read each one, decide how much it is like you and } \\
\text { circle the corresponding number }\end{array}$ & $\begin{array}{l}\text { Very } \\
\text { much } \\
\text { like } \\
\text { me }\end{array}$ & $\begin{array}{l}\text { Mostly } \\
\text { like me }\end{array}$ & $\begin{array}{c}\text { Some } \\
\text {-what } \\
\text { Like } \\
\text { me }\end{array}$ & $\begin{array}{l}\text { A } \\
\text { little } \\
\text { like } \\
\text { me }\end{array}$ & $\begin{array}{l}\text { Not } \\
\text { like } \\
\text { me } \\
\text { at all }\end{array}$ \\
\hline 1 & I ask questions all the time. & 5 & 4 & 3 & 2 & 1 \\
\hline 2 & I stand up to kids who are acting mean. & 5 & 4 & 3 & 2 & 1 \\
\hline 3 & I don't put things off if I can do them straight away. & 5 & 4 & 3 & 2 & 1 \\
\hline 4 & I often come up with different ways of doing things. & 5 & 4 & 3 & 2 & 1 \\
\hline 5 & I do the right thing even if no-one is looking. & 5 & 4 & 3 & 2 & 1 \\
\hline 6 & $\begin{array}{l}\text { I hardly ever have regrets after I have made a } \\
\text { decision }\end{array}$ & 5 & 4 & 3 & 2 & 1 \\
\hline 7 & I am good at getting along with all sorts of people. & 5 & 4 & 3 & 2 & 1 \\
\hline 8 & I am a hard worker. & 5 & 4 & 3 & 2 & 1 \\
\hline 9 & $\begin{array}{l}\text { I get things done that need to get done, even when } \\
\text { I don't feel like doing them. }\end{array}$ & 5 & 4 & 3 & 2 & 1 \\
\hline 10 & I often notice beautiful things. & 5 & 4 & 3 & 2 & 1 \\
\hline 11 & I always want to know more. & 5 & 4 & 3 & 2 & 1 \\
\hline 12 & $\begin{array}{l}\text { I stand up for myself and others, even if I know I } \\
\text { will get teased. }\end{array}$ & 5 & 4 & 3 & 2 & 1 \\
\hline 13 & $\begin{array}{l}\text { When I see art or listen to music, I often forget how } \\
\text { much time is passing. }\end{array}$ & 5 & 4 & 3 & 2 & 1 \\
\hline 14 & People say that I am humorous or playful. & 5 & 4 & 3 & 2 & 1 \\
\hline 15 & I keep trying even after I fail. & 5 & 4 & 3 & 2 & 1 \\
\hline 16 & I am good at bringing smiles to people. & 5 & 4 & 3 & 2 & 1 \\
\hline 17 & I usually understand how I feel and why. & 5 & 4 & 3 & 2 & 1 \\
\hline 18 & $\begin{array}{l}\text { I am good at knowing what people want without } \\
\text { asking. }\end{array}$ & 5 & 4 & 3 & 2 & 1 \\
\hline 19 & $\begin{array}{l}\text { Seeing beautiful pictures or listening to beautiful } \\
\text { music makes me feel better. }\end{array}$ & 5 & 4 & 3 & 2 & 1 \\
\hline 20 & I always like to do things in different ways. & 5 & 4 & 3 & 2 & 1 \\
\hline 21 & $\begin{array}{l}\text { I often share thoughts and feelings with those close } \\
\text { to me. }\end{array}$ & 5 & 4 & 3 & 2 & 1 \\
\hline 22 & I like to tell jokes or funny stories. & 5 & 4 & 3 & 2 & 1 \\
\hline 23 & I enjoy creating new things. & 5 & 4 & 3 & 2 & 1 \\
\hline 24 & I do not pretend to be ok when I am not & 5 & 4 & 3 & 2 & 1 \\
\hline 25 & I speak up for what is right, even when I am afraid. & 5 & 4 & 3 & 2 & 1 \\
\hline 26 & $\begin{array}{l}\text { I am always curious about people, places, or things } \\
\text { I am not familiar with. }\end{array}$ & 5 & 4 & 3 & 2 & 1 \\
\hline 27 & I tell the truth, even if it gets me in trouble. & 5 & 4 & 3 & 2 & 1 \\
\hline
\end{tabular}




\section{Appendix L:}

\section{Flow Chart, From PAL Group Book}

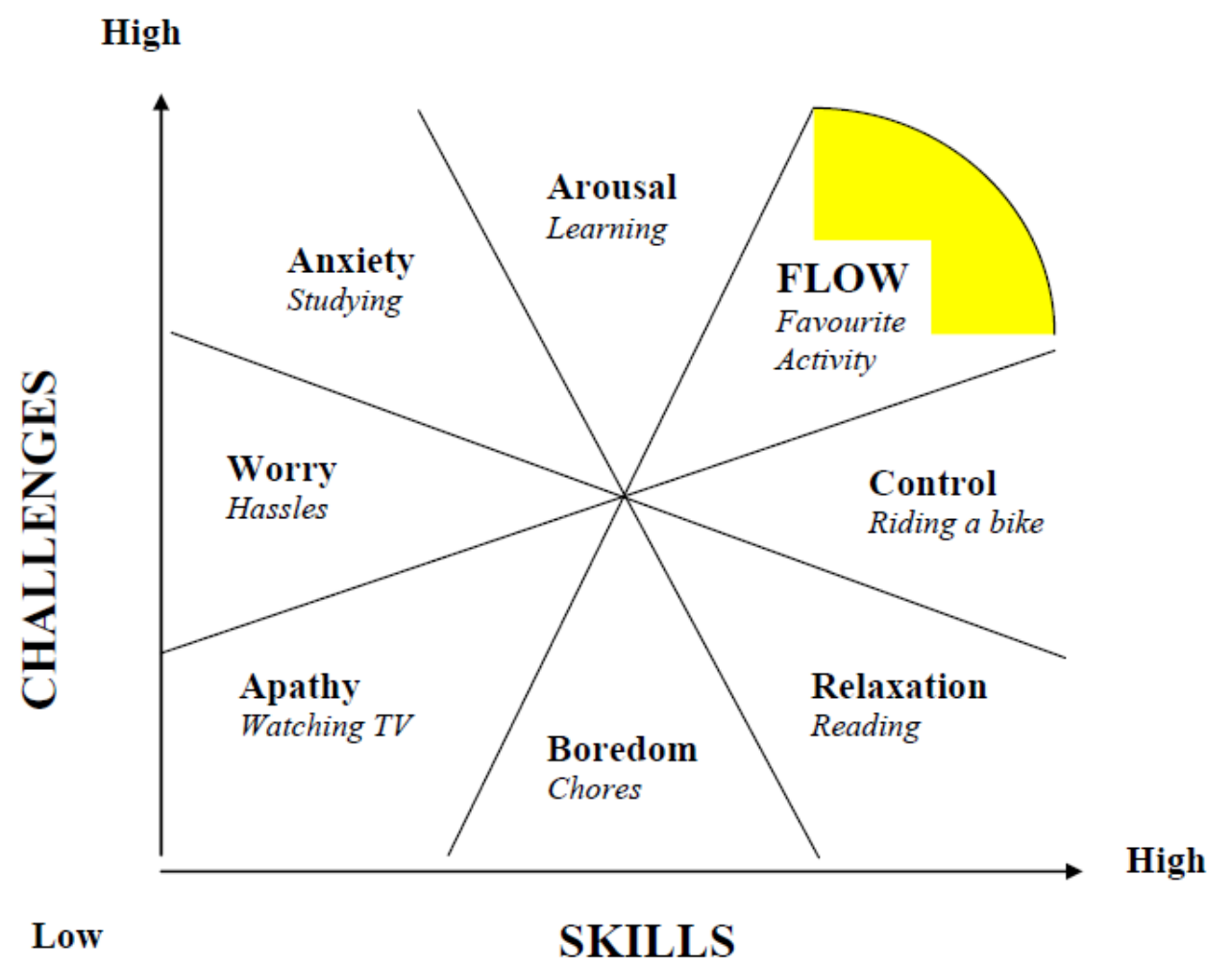

How we experience flow:

1. Timelessness: You're completely focused on the present and hours seem to pass by in minutes.

2. Alive: You feel more alive than usual

3. Motivation: the feeling you get from this activity is reward enough, you do this activity for the activity alone

4. No worries: You are not worried about yourself, others or anything else.

\section{Conditions that help create flow:}

1. Clear goals: The activity you are involved in has a clear set of goals.

2. Balance: There is an equal balance between the challenge of the activity and your skills. (If the challenge is too high you can become stressed if the challenge is too low you can become bored).

3. Concentration: You are so focused on the activity that you block out everything else. 


\section{Appendix M:}

Flow Chart, From PAL Group Book

\begin{tabular}{|c|c|c|c|c|c|c|c|c|}
\hline Area of life & Activity & Clear Goals & $\begin{array}{l}\text { It's a challenge, } \\
\text { but I have the } \\
\text { skills to take it } \\
\text { on }\end{array}$ & $\begin{array}{c}\text { Completely } \\
\text { concentrated }\end{array}$ & $\begin{array}{c}\text { Time } \\
\text { does not } \\
\text { pass in } \\
\text { the usual } \\
\text { way }\end{array}$ & $\begin{array}{l}\text { Enjoyable, } \\
\text { I feel alive }\end{array}$ & $\begin{array}{l}\text { Worth } \\
\text { doing just } \\
\text { for fun }\end{array}$ & $\begin{array}{c}\text { Total Flow } \\
\text { Score }\end{array}$ \\
\hline SPORT & Skating & 1 & 1 & 1 & 1 & 1 & 1 & 6 \\
\hline SCHOOL & Writing & 1 & 1 & 0 & 0 & 1 & 1 & 4 \\
\hline HOBBIES & Fire-twirling & 1 & 0 & 1 & 1 & 1 & 1 & 5 \\
\hline OTHER PEOPLE & Conversation & 0 & 0 & 1 & 1 & 1 & 1 & 5 \\
\hline DAILY TASKS & Walking & 1 & 0 & 0 & 1 & 1 & 1 & 4 \\
\hline
\end{tabular}

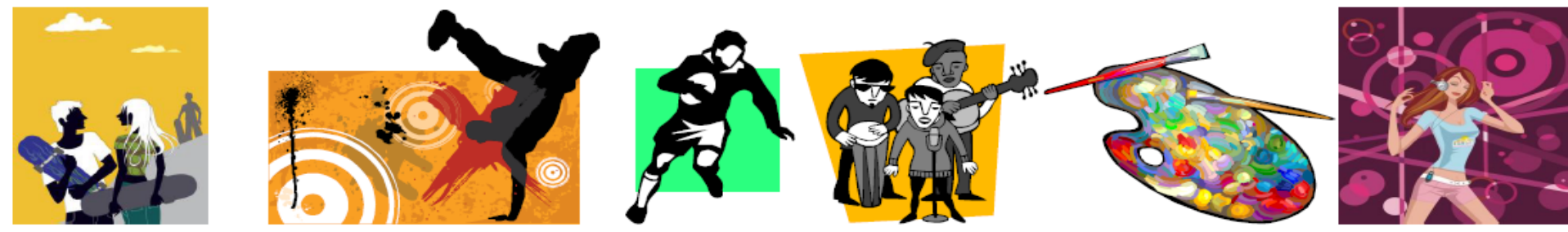




\section{Appendix N: \\ Savouring Activities, From PAL Group Book}

Pick one activity to do during the week and test if savouring it increases your happiness.

Savour and reminisce with others. You can increase your joy in life if you share it with others. Appreciate the present moment with a friend or discuss a shared memory.

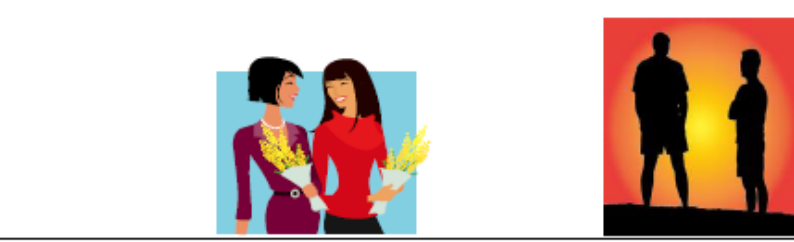

Be open to beauty. When you see something beautiful, take time to appreciate it.

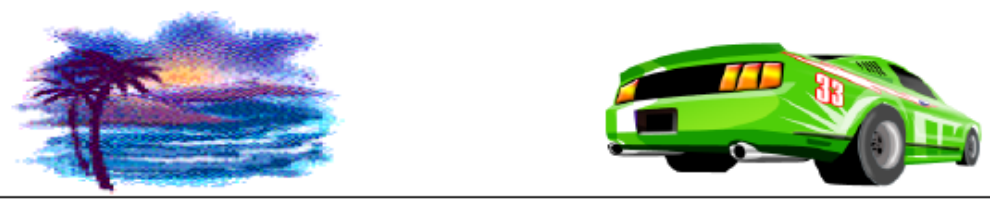

Celebrate good news. When something good happens to you, celebrate it with others to help you savour more deeply.
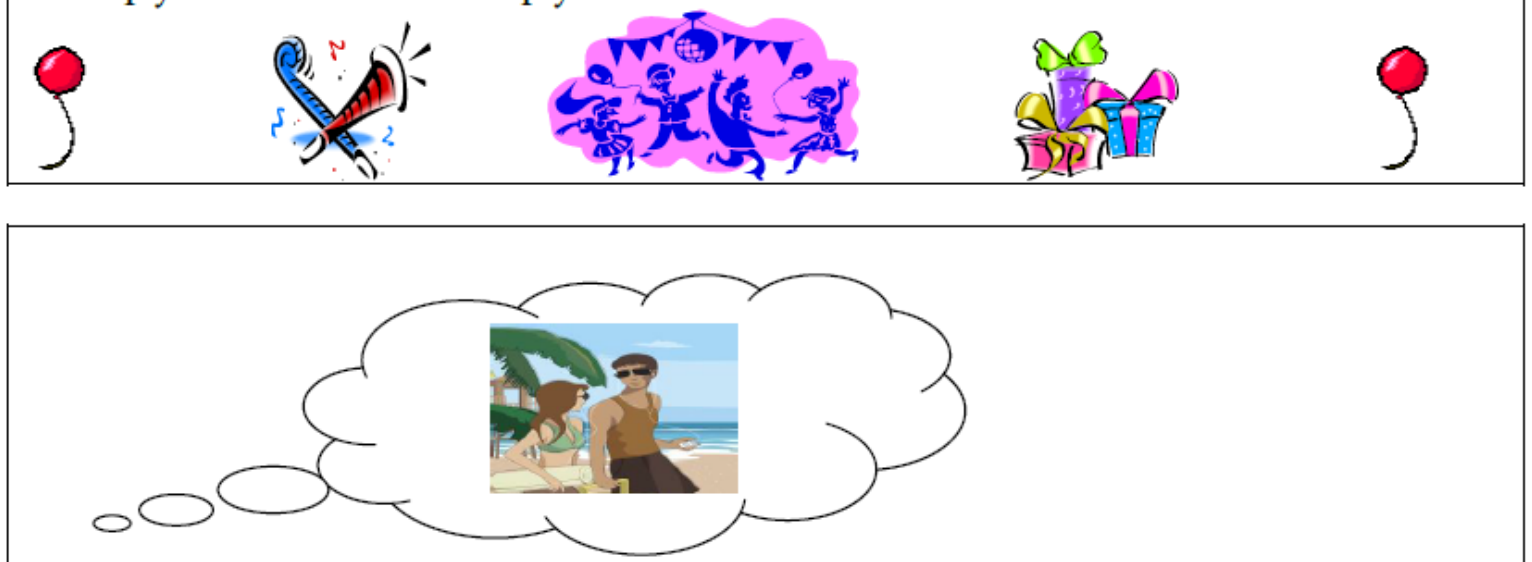

Replay happy events to yourself. Remember moments or days when you were really happy and relive them. 


\section{Appendix $\mathbf{N}$ continued}

Take photos - this can help you to savour many happy moments in your life.
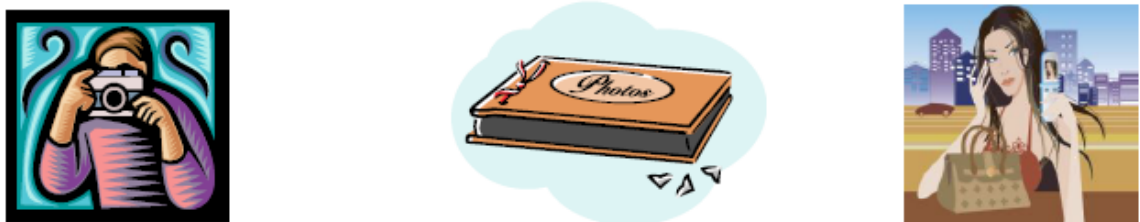

Create a journal - write down moments you want to savour
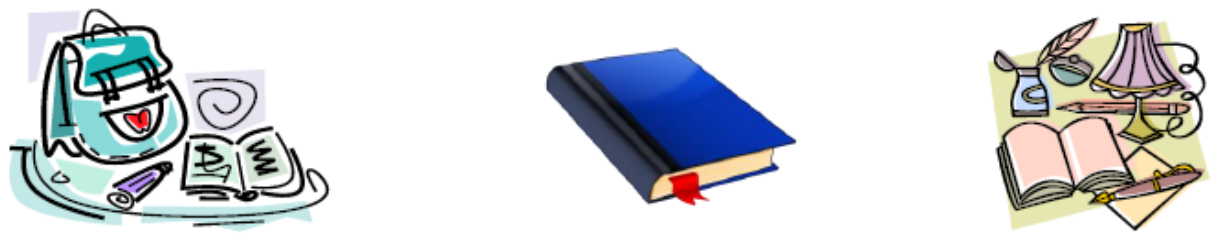

Create an album and put in pictures that you want to savour - these can be drawings, paintings, magazine pictures or photos of happy memories
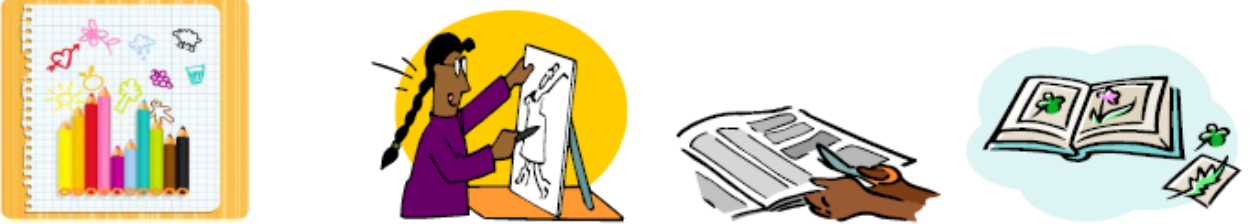


\section{Appendix 0: \\ Goal Setting Rules, From PAL Facilitator's Handbook}

1. Realistic - you have to be physically able to achieve the goal.

2. Make the goal challenging .

3. It has to be your own goal - you have to choose it and you have to be comfortable with it.

4. The goal has to be important to you - it has to mean something to you and be rewarding for you.

5. The goal should aim to gain something, rather than avoid something To start mountain biking rather than to stop being lazy.

6. Chose a goal that will continue/ have unending activity, e.g. to join a netball team rather than just buying a new netball. 


\section{Appendix P:}

Goal Setting Action Plan, From PAL Group Book

\section{ACTION PLAN - Achieving your goal}

Answer the following questions:

Do you want to achieve your goal by a certain date? If so, when?

What resources will you need in order to achieve your goal?

Can you break up your goal into smaller steps or parts? If so, list them below:

What activities will help me achieve my goal?

Identify an activity you can do during the next week that will help you to achieve your main goal

Who would support you in achieving this goal? 


\section{Appendix Q: \\ Acts of Kindness Ideas, From PAL Group Book}

- Leave a small gift at the door of a family.

- Write a note to a teacher thanking him or her for a lesson.

- Send a nice card anonymously to a person that you know is feeling down.

- Visit an animal shelter and bring some treats for the animals and the staff.

- Do a job (like mowing the lawn, cleaning the house) for someone who is having hard times and surprise him or her by not charging.

- Let the person behind you go ahead of you in line.

- Bake biscuits or another treat and give them away at school.

- Bring extra pens/pencils to school and give them away to people who forget to bring one.

- Hug a friend or family member.

- Do something for others who usually do something for you. Clean up the living room, make breakfast on Sunday, or do the laundry.

- Take a new or transfer student and show them around the school.

- Spend an afternoon visiting residents at a senior centre or nursing home.

- Look for the good in everyone you meet.

- Help someone struggling with heavy bags.

- Compliment someone about something he or she is wearing.

- Smile at people you pass on the street or on the way to class.

- Write anonymous positive notes to people saying what makes the recipient special.

- Give someone a flower or a lollipop to show you care.

- Invite someone new to your home to hang out.

- Volunteer at a shelter, a hospital, or a non-profit organization.

- Cook a surprise dinner for your parent/s, complete with candles and a menu.

- Stick up for someone who is getting picked on.

- Eat lunch with someone who is new or sitting alone, or invite him/ her to your table.

- Help a student with a computer problem or a tough homework assignment.

- Help an elderly neighbour with household chores that they are struggling with.

- Talk to a student you've never talked to before.

- Unload the dishwasher or do the dishes without being asked.

- Tell or write your friends why they are important to you. 


\section{Appendix R:}

PAL Idea Sheet, From PAL Facilitator's Handbook

\section{Gratefulness}

Testing the theory of gratefulness:

Research suggests that being grateful increases happiness

\section{You have 10 minutes to prepare and 5 minutes to present a lesson on Gratefulness}

The aim of this activity is for your group to remind everyone about the discussion and activities you had on gratefulness at the beginning of the programme and to add and share any new discoveries or ideas about gratefulness. (If you have time you can add how acts of kindness are related to gratefulness).

Your group can do this in any way that you like You can share stories, do a role-play, rap, draw, sing...

Some important points on gratitude that you might want to include:

- Gratefulness is about recognising the good and beneficial things that happen to us.

- The more grateful we are the happier we become about the people and things in our lives.

- We can show our gratitude in so many different ways: gifts, doing chores, writing thank you letters/cards etc.

- When we show our gratitude it can make others want to do the same - it's like a chain reaction.

- When we are going through difficult times sometimes looking for something to be grateful about can make us feel better. 


\section{Appendix $R$ continued}

\section{Character Strengths}

Research suggests that people who know and practice their strengths feel more in control of their lives and are happier than those that do not think about their strengths.

You have 10 minutes to prepare and 5 minutes to present a lesson on Character Strengths

The aim of this activity is for your group to remind everyone about the discussion and activities you had on character strengths at the beginning of the programme and to add and share any new discoveries or ideas about strengths. (If you have time you can add how character strengths are related to many other lessons we had such as problem solving and self-esteem).

Your group can do this in any way that you like You can share stories, do a role-play, rap, draw, sing...

Some important points on character strengths that you might want to include:

- A character strength is a way of doing things that comes naturally to you.

- Using our character strengths can make us feel satisfied and happy about what we are doing.

- Being aware of our character strengths and other people's strengths can help us understand ourselves and each other 


\section{Appendix $R$ continued}

\section{(1) Savouring and Flow s-is}

Research suggests that people who savour things are more likely to be happier and less stressed than those who don't savour.

Research suggests that experiencing flow increases selfconfidence, satisfaction in life and makes people happy.

You have 10 minutes to prepare and 5 minutes to present a lesson on Savouring and Flow

The aim of this activity is for your group to remind everyone about the discussion and activities you had on savouring and flow and to add and share any new discoveries or ideas about savouring or flow.

Your group can do this in any way that you like You can share stories, do a role-play, rap, draw, sing...

Some important points on savouring that you could include:

- Savouring is about making the most of an enjoyable experience.

- Savouring can include thinking about memories, enjoying something right now or looking forward to something in the future.

- There are many activities we can do to savour including taking photos, sharing enjoyable experiences with friends, and writing things down that you want to savour.

Some important points on flow that you could include:

- Flow is being so completely absorbed in an activity that you forget about the time, you forget your worries and you feel so great that you could keep doing the activity forever.

- For a person to experience flow it is important that there are clear goals and that there is a balance between their skill and the challenge of the activity (it can't be too easy or too difficult for the person). They need to have their full concentration on the activity.

- Some activities that people experience flow in include: sports, painting, writing, dancing, reading, and playing an instrument. 


\section{Appendix S}

Table of comparison between PAL and Kiwi ACE

\begin{tabular}{|c|c|c|}
\hline Sessions & Kiwi ACE & PAL \\
\hline 1 & Intro - Stress and my body & Intro - Variety of emotions \\
\hline 2 & Thinking and feeling & Practicing gratitude \\
\hline 3 & Thoughts aren't facts & Identifying strengths \\
\hline 4 & Detecting evidence & Enjoying life \\
\hline 5 & Problem solving & Problem solving \\
\hline 6 & Changing habits & Goal Setting \\
\hline 7 & Social Skills & Building Relationships \\
\hline 8 & Assertiveness & Assertiveness \\
\hline 9 & Negotiation \& compromise & Negotiation \& compromise \\
\hline 10 & Deep down beliefs & Liking who I am \\
\hline 11 & Review of Kiwi ACE & Review of PCP \\
\hline 12 & Questionnaire & Questionnaire \\
\hline
\end{tabular}

